\title{
Light Trapping in Plasmonic Solar Cells
}

\author{
Thesis by \\ Vivian Eleanor Ferry
}

In Partial Fulfillment of the Requirements

for the Degree of

Doctor of Philosophy

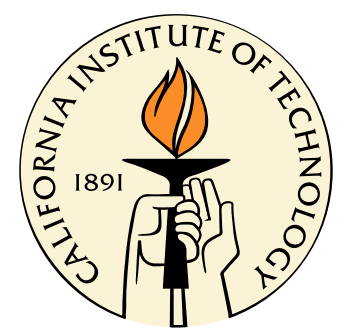

California Institute of Technology

Pasadena, California

2011

(Defended May 6, 2011) 
(c) 2011

Vivian Eleanor Ferry

All Rights Reserved 
In memory of Abe

and especially his love of science and education 


\section{Acknowledgements}

I am tremendously grateful to all of the people who have supported me during my time at Caltech, providing advice, assistance, and encouragement along the way. I feel extremely fortunate to have worked with so many extraordinary people.

Most importantly, thank you to my advisor Harry Atwater for his incredible support. Harry is full of enthusiasm, ideas, and inspiration, and I am grateful for all of the things he has taught me, both in research and personally. No matter how long the days, Harry has always been focused and willing to help, either with research creativity or with encouragement. I would also like to thank the members of my committee, Jackie Barton, Harry Gray, and Nate Lewis, for their advice over the years.

I am also deeply grateful to Albert Polman, who has in many ways served as a second advisor to me. I spent several months at AMOLF over repeat visits, and Albert has been a constant source of research ideas and insightful questions. I particularly admire his ability to find and clearly express the novel aspects of any experiment, and I hope to emulate this approach in the future.

I have been fortunate to collaborate with many institutions and groups throughout my graduate school experience. In particular, Ruud Schropp at Utrecht University has provided many scientific ideas and perspective on the applications of nanophotonics to solar energy. He graciously agreed to deposit the solar cells used in this thesis. Peter Saeta, from Harvey Mudd College, has been an enthusiastic modeling collaborator, particularly during his sabbatical visit to Caltech.

I am especially grateful to those who provided support in my first few years, especially Luke Sweatlock, who taught me everything I know about numerical calculations, and has continued to be a fantastic resource and collaborator. Domenico Pacifici was particularly helpful in the beginning, both experimentally and in bringing my chemistry background up to speed in solid-state physics. Imogen Pryce has been my Caltech "other half" for these last 5 years, acting as a sounding board and editor many times. Koray Aydin, Ryan Briggs, Dennis Callahan, Ken Diest, Jen Dionne, Carrie Hofmann, Jeremy Munday, and Deirdre O'Carroll all contributed many ideas 
in plasmonics. I would additionally like to thank Krista Langeland for her help with Si light trapping, Mike Kelzenberg for his help with calculations, Mike Filler for being the best officemate a first year graduate student could ask for, and my SURF student Dahvyd Wing. To those not mentioned here, I am no less thankful - it would be impossible to mention all of your contributions.

The many members of the Atwater group have made the group a diverse and fun place to be for the last several years. Thank you to all of the members I have overlapped with (alphabetically): Melissa Archer, Jeff Bosco, Stanley Burgos, Chris Chen, Naomi Coronel, Davis Darvish, Mike Deceglie, Matt Dicken, Carissa Eisler, Hal Emmer, Jim Fakonas, Eyal Feigenbaum, Kate Fountaine, Jonathan Grandidier, Min Jang, Seokmin Jeon, Brendan Kayes, Seyoon Kim, Greg Kimball, Emily Kosten, Lise Lahourcade, Andrew Leenheer, Marina Leite, Manav Malhotra, Gerald Miller, Keisuke Nakayama, Morgan Putnam, Matt Sheldon, Amanda Shin, Faisal Tajdar, Adele Tamboli, Katsu Tanabe, Yulia Tolstova, Dan Turner-Evans, Rene de Waele, Robb Walters, Emily Warmann, Raymond Weitekamp, and Sam Wilson. Our wonderful administrative assistants have made all aspects of the group easier: thanks to April Neidholdt, Lyra Haas, Jennifer Blankenship, and Tiffany Kimoto.

Much of my research was performed in the Netherlands, and I am grateful to the many collaborators who made this possible. Marc Verschuuren has been a fantastic collaborator, and his beautiful structures enabled the experimental measurements in the second part of this thesis. Claire van Lare and Hongbo Li both spent many hours in Utrecht doing measurements with me. Robb Walters and Ewold Verhagen both contributed with experimental and calculation assistance. In Utrecht, Karine van der Werf performed the cell depositions. Thank you to the rest of the group at AMOLF for their scientific support and welcoming me to the group: Toon Coenen, Jorik van der Groep, Maarten Hebbink, Erwin Kroekenstoel, Martin Kuttge, Ruben Maas, James Parsons, Hinke Schokker, Piero Spinelli, Rutger Thijssen, and Ernst Jan Vesseur.

I am thankful to my family and friends for the support. My parents, Steve and Audrey Ferry, and my brother Ken Ferry, have been excited to hear about research, 
vii

and also provided occasional mathematical and programming help. In addition they provided much needed breaks from work and personal support. My parents in-law, John and Andree Valley, have been supportive and welcoming to the family, and George and Marcy Valley have made the move to Los Angeles much easier by essentially adopting us. I am also grateful to many friends as close as family, especially Indira Sriram and Elisa Pueschel.

And finally, thank you to my husband and partner of the last nine years, David, for his love, support, four am pep talks, graphic design and cooking skills, and amazing sense of humor.

Vivian E. Ferry April 2011

Pasadena, $C A$ 


\section{Abstract}

Subwavelength nanostructures enable the manipulation and molding of light in nanoscale dimensions. By controlling and designing the complex dielectric function and nanoscale geometry we can affect the coupling of light into specific active materials and tune macroscale properties such as reflection, transmission, and absorption. Most solar cell systems face a trade-off with decreasing semiconductor thickness: reducing the semiconductor volume increases open circuit voltages, but also decreases the absorption and thus the photocurrent. Light trapping is particularly critical for thin-film amorphous Si (a-Si:H) solar cells, which must be made less than optically thick to enable complete carrier collection. By enhancing absorption in a given semiconductor volume, we can achieve high efficiency devices with less than $100 \mathrm{~nm}$ of active region.

In this thesis we explore the use of designed plasmonic nanostructures to couple incident sunlight into localized resonant modes and propagating waveguide modes of an ultrathin semiconductor for enhanced solar-to-electricity conversion. We begin by developing computational tools to analyze incoupling from sunlight to guided modes across the solar spectrum and a range of incident angles. We then show the potential of this method to result in absorption enhancements beyond the limits for thick film solar cells. The second part of this thesis describes the integration of plasmonic nanostructures with a-Si:H solar cells, showing that designed nanostructures can lead to enhanced photocurrent over randomly textured light trapping surfaces, and develops a computational model to accurately simulate the absorption in these structures. The final chapter discusses the fabrication of a high-efficiency (9.5\%) solar cell with a less than $100 \mathrm{~nm}$ absorber layer and broadband, angle isotropic photocurrent enhancement. Moreover, we discuss general design rules where light trapping nanopatterns are defined by their spatial coherence spectral density. 


\section{Contents}

List of Figures $\quad$ xvii

List of Tables $\quad$ xxiii

List of Publications $\quad$ XXV

1 Introduction 1

1.1 Motivation . . . . . . . . . . . . . . . . . . . 1

1.2 Solar Cell Overview . . . . . . . . . . . . . . . . . . 2

1.3 Solar Cell Fundamentals . . . . . . . . . . . . . . . . . . . . . 3

1.4 Ultrathin-film Solar Cells . . . . . . . . . . . . . . . . . . 6

1.4.1 Availability of Raw Materials . . . . . . . . . . . . . 7

1.4 .2 Throughput ........................ 10

1.4.3 Carrier Collection . . . . . . . . . . . . . . . . . . . 10

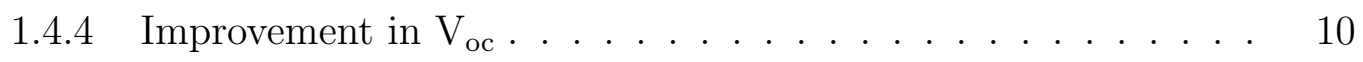

1.4.5 Stability ..................... 11

1.4.6 Absorption and Layer Thickness . . . . . . . . . . . . . . . 11

1.5 Approaches to Light Trapping . . . . . . . . . . . . . . . . . . . . 13

1.5.1 Ergodic Limit . . . . . . . . . . . . . . . 13

1.5.2 Thin-films ..................... . . . 14

1.6 Plasmonics . . . . . . . . . . . . . . . . . . . . 16

1.7 Plasmonic Photovoltaics . . . . . . . . . . . . . . . 17

1.8 Overview of Thesis . . . . . . . . . . . . . . . . . . . 19 
2 Plasmonic Solar Cells $\quad 21$

2.1 Introduction . . . . . . . . . . . . . . . . . . . . . 21

2.2 Overview of Mechanisms for Plasmonic Absorption Enhancement . . 21

2.3 Plasmonic Scattering . . . . . . . . . . . . . . . . . 24

2.4 Waveguide Modes . . . . . . . . . . . . . . . . . . 29

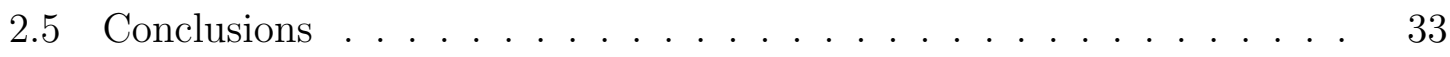

3 Numerical Methods $\quad 35$

3.1 Introduction . . . . . . . . . . . . . . . . . . 35

3.2 Fourier Mode Spectral Analysis . . . . . . . . . . . . . . . 36

3.2.1 Isolation of Scattered Fields . . . . . . . . . . . . . . 38

3.2 .2 Fourier-Space Mode Filtering . . . . . . . . . . . . . . . 40

3.2.3 Incoupling Cross Sections . . . . . . . . . . . . . . . . 44

3.2.4 FMSA with Broadband Sources . . . . . . . . . . . . . 47

3.3 Absorption . . . . . . . . . . . . . . . . . . . . . . 48

3.4 Conclusions ........................... 52

4 Incoupling from Single Isolated Nanostructures $\quad 53$

4.1 Introduction . . . . . . . . . . . . . . . . . . 53

4.2 Resonances Affecting Incoupling to Modes . . . . . . . . . . . . . . 54

4.3 Ridges vs. Grooves . . . . . . . . . . . . . . . . . . . 61

4.4 Absorption Enhancements from Single Scattering Objects . . . . . . . 63

4.5 Angle Dependence . . . . . . . . . . . . . . . 66

4.6 Arrays of Scattering Elements . . . . . . . . . . . . . 66

4.7 Conclusions . . . . . . . . . . . . . . . . . . . . 70

5 How Much Do Guided Modes Enhance Absorption in Thin Semi$\begin{array}{ll}\text { conductor Volumes? } & 71\end{array}$

5.1 Introduction . . . . . . . . . . . . . . . . . . . 71

5.2 Method ........................ 73

5.2.1 Absorption in Guided Modes . . . . . . . . . . . . . 74 
xiii

5.2 .2 Dipole Scattering . . . . . . . . . . . . . . . . . . . 78

5.2 .3 Multiple Scattering . . . . . . . . . . . . . . . . . . 81

5.3 Results . . . . . . . . . . . . . . . . . . . . . 83

5.3.1 Comparison to the Ergodic Limit . . . . . . . . . . . . 85

5.3 .2 Comparing Semiconductors _. . . . . . . . . . . . 89

5.4 Assumptions of the Model . . . . . . . . . . . . . . . . . . . . . . 91

5.5 Conclusion . . . . . . . . . . . . . . . . . . . . . . . . . . 93

$5.6 \quad$ Appendix . . . . . . . . . . . . . . . . . . . . . . . . . . 93

6 Improved Red Response in a-Si:H Solar Cells with Soft-Imprinted $\begin{array}{ll}\text { Back Reflectors } & 97\end{array}$

6.1 Introduction . . . . . . . . . . . . . . . . . . . . 97

6.2 Advantages of Light Trapping for a-Si:H _ . . . . . . . . . 97

6.3 Nanofabrication: Substrate Conformal Imprint Lithography . . . . . . 100

6.4 Cell Fabrication . . . . . . . . . . . . . . . . . . . . . . . . . . . . 103

6.5 Improving Red Response of a-Si:H Solar Cells with Hole Arrays . . 104

6.6 Fabrication of Pillar Array Solar Cells _ . . . . . . . . . . . 107

6.7 Comparison of Periodic Structures to Random Textures . . . . . . . . 111

6.7.1 Efficiency Measurements . . . . . . . . . . . . . . . . 111

6.7.2 External Quantum Efficiency Measurements . . . . . . . . 115

6.8 Angle-Resolved Photocurrent . . . . . . . . . . . . . . . . . 115

6.9 Conclusion . . . . . . . . . . . . . . . . . . . . . . . . . . . . . . . . . . . . . . 121

7 Simulation of Ultrathin-Film a-Si:H Solar Cells: Comparison to Experimental Measurements and Optimization 123

7.1 Introduction . . . . . . . . . . . . . . . . . . . . . 123

7.2 Simulation of Fabricated Cells . . . . . . . . . . . . . . . . 124

7.3 Understanding Light Trapping in Periodic Cells . . . . . . . . . 128

7.3.1 Absorption across solar spectrum . . . . . . . . . . . 128

7.3.2 Where Are the Losses? . . . . . . . . . . . . . . . . . . . . . 131

7.3.3 Role of the ZnO:Al Layer _ . . . . . . . . . . . . . . . 132 
xiv

7.3.4 Is the Metal Necessary? . . . . . . . . . . . . . . . . . . . . . 133

7.3.5 Other Metals ................... 136

7.4 Modeling Random Textures . . . . . . . . . . . . . . . . . 136

7.4.1 Role of Curvature . . . . . . . . . . . . . . . . . . . . . 136

7.5 Optimization of Periodic Nanopatterns . . . . . . . . . . . . 137

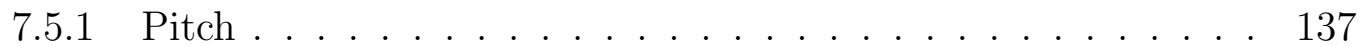

7.5 .2 Hexagonal Lattice . . . . . . . . . . . . . . . . . . . . . . . . 140

7.5.3 Pitch and Diameter . . . . . . . . . . . . . . . . . . . 142

7.6 Conclusions . . . . . . . . . . . . . . . . . . 143

8 The Role of Spatial Frequency: High Efficiency a-Si:H Solar Cells 145

8.1 Introduction . . . . . . . . . . . . . . . . . . . 145

8.2 Design and Fabrication of Engineered Random Arrays . . . . . . . . . 147

8.2.1 Calculation of Power Spectral Density . . . . . . . . . . . . . 149

8.2.2 Fabrication of Replica Asahi Substrates . . . . . . . . . . . . . 152

8.3 Solar Cell Measurements . . . . . . . . . . . . . . . . . . . 153

8.3.1 Characterization of Topography . . . . . . . . . . . 153

$8.3 .2 \mathrm{~J}-\mathrm{V}$ Measurements . . . . . . . . . . . . . . . . 155

8.3.3 EQE Measurements . . . . . . . . . . . . . . . 160

8.3.4 Angle-Resolved EQE Measurements . . . . . . . . . . . . . . 163

8.3.5 Simulation of Measured Structures . . . . . . . . . . . 165

8.4 Connection to PSD Calculations . . . . . . . . . . . . . . . . . 169

8.5 Mie Scattering from Top Interface . . . . . . . . . . . . . . . . 171

8.6 Conclusions . . . . . . . . . . . . . . . . . . . 173

9 Summary and Outlook $\quad 175$

9.1 Summary . . . . . . . . . . . . . . . . . . . 175

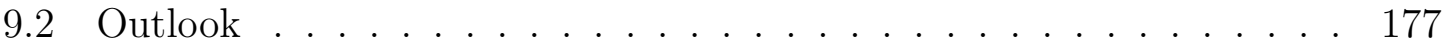

9.2.1 Fabrication of p-i-n Cells . . . . . . . . . . . . . . . 177

9.2.2 Application to Multijunction Cells . . . . . . . . . . 178

9.2.3 Application to other Materials Systems . . . . . . . . . . . . 180 
$\mathrm{XV}$

9.2.4 Effect of Nanostructures on Voc and FF . . . . . . . . . . . . 182

9.3 Conclusions . . . . . . . . . . . . . . . . . . . . 183

$\begin{array}{ll}\text { Bibliography } & 184\end{array}$ 
xvii

\section{List of Figures}

1.1 Photon energy compared to bandgap of a semiconductor. . . . . . . . 3

1.2 Basic quantities for solar cell characterization: J-V curves and EQE

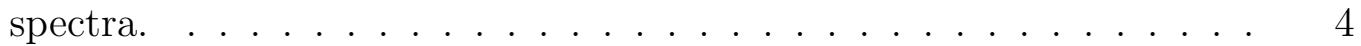

1.3 Schematics of direct and diffuse angles of incident sunlight striking a solar cell. . . . . . . . . . . . . . . . . . . . . . . . . 7

1.4 Fraction of the AM1.5 solar spectrum absorbed in a single pass through c-Si layers of varying thickness. . . . . . . . . . . . . . . . . . . 12

1.5 Approaches to light trapping in thin-films. . . . . . . . . . . . . . . . 14

2.1 Overview of plasmonic light trapping structures. . . . . . . . . . . . . 22

2.2 Parameter space for plasmonic solar cell design. . . . . . . . . . . 23

2.3 Diffusion model for light propagation inside the solar cell. . . . . . . . 27

2.4 Modal profiles at $\lambda=920 \mathrm{~nm}$ for TM and TE polarizations. . . . . . 30

2.5 Surface plasmon polariton dispersion relation for an Ag/Air interface. 32

3.1 Schematic of sample structure. . . . . . . . . . . . . . . . 37

3.2 Isolation of scattered fields via subtraction. . . . . . . . . . . . 39

3.3 Calculated scattered $H_{y}$ fields . . . . . . . . . . . . . . . 41

3.4 FMSA method for isolating waveguide modes. . . . . . . . . . . . . 42

$3.5 H_{y}$ field maps of isolated modes after filtering . . . . . . . . . . . . 43

3.6 Schematic of calculation of power flow away from the scattering object. 44

3.7 Calculation of incoupling cross sections. . . . . . . . . . . . . 46

3.8 Multimode dispersion relation calculated from FMSA method. . . . . 47 
xviii

3.9 AM1.5 solar spectrum with wavelengths of simulations marked. . . . 49

3.10 Generation rate maps for thick and thin c-Si. . . . . . . . . . . 50

3.11 Solar spectrum weighted generation rate. . . . . . . . . . . 51

4.1 Dispersion relations for three-layer waveguide: Ag/c-Si/air. . . . . . 54

4.2 Schematic of groove cut into Ag back contact on thin film of c-Si. . . 55

4.3 Calculated incoupling cross sections across the spectrum. . . . . . . 56

4.4 Incoupling cross sections as a function of width and depth of a groove. 60

4.5 Incoupling cross sections for a ridge. . . . . . . . . . . . . . 61

4.6 Angular backscattering from a groove and ridge. . . . . . . . . . . 62

4.7 Absorption enhancement from a single groove. . . . . . . . . . . . . . 64

4.8 Absorption enhancement as a function of angle of incidence. . . . . . 66

4.9 Calculated absorption enhancement from arrays of ridges. . . . . . . 67

4.10 Angle-resolved absorption enhancement for an array of grooves. . . . 69

5.1 A multilayer structure with an antireflection-coated silicon absorbing layer on top of an optically thick layer of silver. . . . . . . . . . . 75

5.2 Absorption enhancement in a silicon layer on silver compared to a single pass at normal incidence. . . . . . . . . . . . . . . . 77

5.3 Normalized decay rate for a dipole in a silicon layer. . . . . . . . . . . 80

5.4 Diffusion model of light propagation inside the multilayer structure. . 81

5.5 Band-edge absorption enhancement of light leaving a dipole compared to single pass absorption. . . . . . . . . . . . . . . . 84

5.6 Absorption enhancement of light near the band edge caused by dipoles parallel to the surface of an $800 \mathrm{~nm}$ Si layer on Ag. . . . . . . . . . . 86

5.7 Modal profiles in an $800 \mathrm{~nm}$ Si layer on Ag at $\lambda=1100 \mathrm{~nm}$. . . . . 88

5.8 Limiting integrated absorption efficiency (IAE) for thin layers of silicon, germanium, amorphous silicon, and gallium arsenide on Ag illuminated with the AM1.5G spectrum. . . . . . . . . . . . . . 90

6.1 SCIL stamp fabrication. . . . . . . . . . . . . . . . 101 
xix

6.2 Overview of SCIL imprint process. . . . . . . . . . . . . 103

6.3 SEM image of a nanoimprinted pattern of holes after overcoating with Ag. . . . . . . . . . . . . . . . . . . . . . . 104

6.4 SEM cross section images of solar cell deposited over hole array. . . . 105

6.5 Best JV measurements of the flat reference and hole array patterned a-Si:H cells. . . . . . . . . . . . . . . . . . . . . . 106

6.6 Measured EQE spectra for flat reference cells and hole array patterned a-Si:H cells.. . . . . . . . . . . . . . . . . . . . . . . 107

6.7 Photographs of fully fabricated solar cells over nanopatterned substrates. 108

6.8 SEM images of Ag-coated square periodic arrays with varying pitch and diameter. . . . . . . . . . . . . . . . . . . 109

6.9 SEM images of Ag-coated staggered periodic arrays. . . . . . . . . . . 109

6.10 FIB cross section of cell with $115 \mathrm{~nm}$ i-layer deposited over nanopattern with $500 \mathrm{~nm}$ pitch. . . . . . . . . . . . . . . . 110

6.11 Surface topography of nanopatterned and randomly textured solar cells.111

6.12 Electrical measurements on plasmonic solar cells. . . . . . . . . . . . 112

6.13 External quantum efficiency spectra of nanopatterned and randomly textured cells from measurement and simulation. . . . . . . . . . . . 116

6.14 EQE measurements on several different cells with the same nanopattern for repeatability. . . . . . . . . . . . . . . . . . . . 117

6.15 EQE measurements on cells with $500 \mathrm{~nm}$ pitch periodic nanopattern

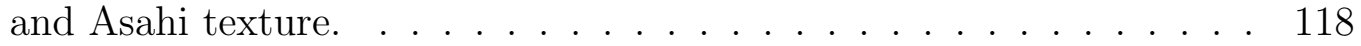

6.16 ngle-resolved photocurrent spectroscopy. . . . . . . . . . . . . . . . 119

7.1 Complex refractive index for a-Si:H, ITO, and $\mathrm{ZnO}: \mathrm{Al}$, as measured with ellipsometry. . . . . . . . . . . . . . . . 125

7.2 Schematic of conformal nanopatterned cell as modeled in simulation. 126

7.3 AFM images of Ag-coated Asahi U-type glass used for simulation. . . 127

7.4 External quantum efficiency spectra of nanopatterned and randomly textured cells from measurement and simulation. . . . . . . . . . . . 129 
$\mathrm{XX}$

7.5 Solar spectrum-weighted photon flux maps for periodic cells. . . . . . 130

7.6 Generation rate maps at different wavelengths spanning the solar spec-

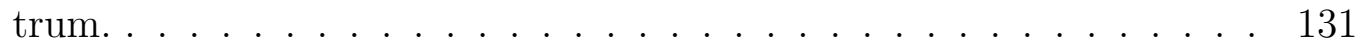

7.7 Calculated absorption in all of the layers of an example cell with 300 nm Ag particles, $500 \mathrm{~nm}$ pitch, and $115 \mathrm{~nm}$ thick intrinsic layers. . . 132

7.8 Dispersion relations with varying $\mathrm{ZnO}$ :Al thickness. . . . . . . . . . . 134

7.9 Calculated generation rate for Ag back reflector with and without plasmonic nanostructures. . . . . . . . . . . . . . . . . 135

7.10 Photon flux calculated at $\lambda=630 \mathrm{~nm}$. . . . . . . . . . . 135

7.11 Calculated generation rates for identical cells with different back contact materials. . . . . . . . . . . . . . . . . . . . . 137

7.12 Calculated photon flux for cells with 115 nm thick intrinsic layers. . . 138

7.13 Calculated generation rates with varying pitch. . . . . . . . . 139

7.14 Calculated generation rates with varying pitch, for a hexagonal lattice. 141

7.15 Optimization of solar spectrum integrated generation rate with covarying diameter and pitch. . . . . . . . . . . . . . . . . . . . . 142

8.1 AFM images and fourier transforms of the Ag-coated patterns used in the experiments. . . . . . . . . . . . . . . . . 148

8.2 Calculation of two-dimensional power spectral density from surface topography. . . . . . . . . . . . . . . . 150

8.3 Power spectral density calculated from AFM data of Ag-coated surfaces.151

8.4 AFM comparison of original Asahi U-type texture and replica texture in silica sol-gel. . . . . . . . . . . . . . . . . . . . 153

8.5 Power spectral density calculations comparing the original Asahi Utype texture and replica texture in silica sol-gel. . . . . . . . . . . . 154

8.6 EQE measurements of cells grown on Asahi U-type glass and replicated Asahi U-type texture. . . . . . . . . . . . . . . . . . 155

8.7 SEM cross sections of solar cells deposited over various light trapping

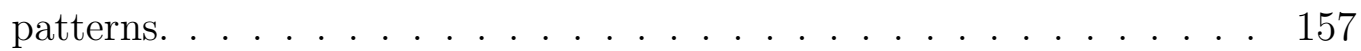


$\mathrm{xxi}$

8.8 AFM scan of ITO top surface of cells deposited over $400 \mathrm{~nm}$ pitch periodic patterns. . . . . . . . . . . . . . . . 157

8.9 Current-voltage measurements for highly performing cells with $90 \mathrm{~nm}$ thick intrinsic layers. . . . . . . . . . . . . . . . . . . . 158

8.10 EQE data on representative cells with $90 \mathrm{~nm}, 115 \mathrm{~nm}$, and $150 \mathrm{~nm}$ intrinsic regions. . . . . . . . . . . . . . . . . . 161

8.11 Repeatability of EQE data on cells with $115 \mathrm{~nm}$ thick intrinsic regions. 162

8.12 Comparison of EQE spectra from cells on $400 \mathrm{~nm}$ pitch nanopatterns with $300 \mathrm{~nm}$ particles from each deposition run. . . . . . . . . . . . . 162

8.13 EQE spectra for cells with $500 \mathrm{~nm}$ pitch periodic patterns, with varying nanoparticle size, and $115 \mathrm{~nm}$ i-layers. . . . . . . . . . . . . . . 163

8.14 Experimental measurements of angle resolved photocurrent on red side of spectrum for periodic and pseudo-random patterns. . . . . . . . . . 164

8.15 Angle-resolved EQE for cells with $90 \mathrm{~nm}$ thick i-layers on $400 \mathrm{~nm}$ pitch periodic patterns, with polarized incident light. . . . . . . . . . . 166

8.16 Angle-resolved $\mathrm{V}_{\text {oc }}$ for $400 \mathrm{~nm}$ pitch periodic cell with $90 \mathrm{~nm}$ thick intrinsic region. . . . . . . . . . . . . . . . . 167

8.17 Simulated absorption in cells with $90 \mathrm{~nm}$ thick i-layers and various light trapping nanopatterns. . . . . . . . . . . . . . . . 168

8.18 Absorption length of a-Si:H vs. wavelength. . . . . . . . . . . . 169

8.19 Photon flux profiles, calculated from simulation, at different wavelengths, for $400 \mathrm{~nm}$ pitch, replica Asahi, and pseudo-random light trapping patterns. . . . . . . . . . . . . . . . . 170

8.20 Calculated normalized absorption cross section for a hemi-ellipsoidal a-Si:H/ITO scatterer. . . . . . . . . . . . . . . . . . . . 172

8.21 Photon flux profiles, calculated from simulation, at different wavelengths. 173

9.1 Fabrication of superstrates for p-i-n devices. . . . . . . . . . . . . . . 178

9.2 Schematic designs of nanostructured tandem thin-film Si solar cells. . 179 
xxii

9.3 Fraction of power in an SPP mode absorbed in semiconductor. The metal is Al. . . . . . . . . . . . . . . . . . . . 181

9.4 Schematic of a quantum dot solar cell. . . . . . . . . . . . . . . . . . 182 
xxiii

\section{List of Tables}

1.1 Current world production of feedstock materials needed for common PV cells. Without increased feedstock production and recycling, many standard PV technologies cannot sustain significant increases in PV demand. Incorporation of ultrathin-film techniques would enable largescale production of any of the above cells. . . . . . . . . . . . 8

6.1 Summary of JV measurements on cells with $295 \mathrm{~nm}$ thick intrinsic layers. The standard deviations are shown in parentheses. . . . . . . . 113

6.2 Summary of JV measurements on cells with $115 \mathrm{~nm}$ thick intrinsic layers. The standard deviations are shown in parentheses. . . . . . . . 114

8.1 JV measurements on cells with $90 \mathrm{~nm}$ intrinsic layer thickness . . . . 156

8.2 JV measurements on cells with $115 \mathrm{~nm}$ intrinsic layer thickness . . . . 158

8.3 JV measurements on cells with $150 \mathrm{~nm}$ intrinsic layer thickness . . . . 159 
xxiv 


\section{List of Publications}

Portions of this thesis have been drawn from the following publications:

V. E. Ferry, M. A. Verschuuren, C. van Lare, R. E. I. Schropp, H. A. Atwater, A. Polman. Optimized Spatial Correlations for Broadband Light Trapping Nanopatterns in High Efficiency Ultra-thin Film a-Si:H Solar Cells. Submitted, 2011.

V. E. Ferry, M. A. Verschuuren, H. B. T. Li, E. Verhagen, R. J. Walters, R. E. I. Schropp, H. A. Atwater, A. Polman. Light Trapping in Ultrathin Plasmonic Solar Cells. Optics Express, 18(13): A237-A245, 2010.

V. E. Ferry, J. N. Munday, H. A. Atwater. Design Considerations for Plasmonic Photovoltaics. Advanced Materials, 22(43): 4794-4808, 2010.

V. E. Ferry, M. A. Verschuuren, H. B. T. Li, E. Verhagen, R. J. Walters, R. E. I. Schropp, H. A. Atwater, A. Polman. Plasmonic light trapping for thin film a-Si:H solar cells. Proceedings of the 35th IEEE Photovoltaics Specialist Conference, Honolulu, Hawaii, 2010.

V. E. Ferry, M. A. Verschuuren, H. B. T. Li, R. E. I. Schropp, H. A. Atwater, A. Polman. Improved red-response in thin film a-Si:H solar cells with soft-imprinted plasmonic back reflectors. Applied Physics Letters, 95(18): 183503, 2009. 
xxvi

Publications, continued:

P. N. Saeta, V. E. Ferry, D. Pacifici, J. N. Munday, H. A. Atwater. How Much Can Guided Modes Enhance Absorption in Thin Solar Cells? Optics Express, 17(23): $20975,2009$.

V. E. Ferry, L. A. Sweatlock, D. Pacifici, H. A. Atwater. Plasmonic nanostructure design for coupling light into solar cells. Nano Letters, 8(12): 4391-4397, 2008. 
xxvii 
xxviii 


\section{Chapter 1}

\section{Introduction}

\subsection{Motivation}

One of the great challenges facing society today is the supply of low-cost, environmentally friendly energy sources that can meet the growing demands of an expanding population. Photovoltaic power has the potential to meet these needs, since the amount of radiation striking the earths surface is $1.76 \times 10^{5}$ terawatts (TW) and current world usage is estimated at $15 \mathrm{TW}[1]$. With respect to other renewable and nonrenewable energy resources, however, the cost of photovoltaic modules continues to be high, and cost per Watt remains the driving force behind much of photovoltaics research.

Concurrently, nanophotonics has emerged as a widespread area of research, with focus on both fundamentals of light control at the nanoscale and applications to devices. Subwavelength nanostructures enable the manipulation and molding of light in nanoscale dimensions. By controlling and designing the complex dielectric function and nanoscale geometry, the coupling of light into specific active materials can be controlled, and macroscale properties such as reflection, transmission, and absorption can be tuned. Applying the methods of nanophotonics to solar cells allows for the possibility of shrinking the absorbing layers while maintaining high levels of absorption, which enables higher efficiency, low cost, stable photovoltaic devices. 


\subsection{Solar Cell Overview}

Solar cell technology development can be broadly classified by the material composition of the active layer. Historically, crystalline $\mathrm{Si}$ (c-Si) has been the dominant semiconductor for photovoltaics, although thin-film solar cells are gaining an increasing market share. For c-Si approximately $50 \%$ of the total cost is due to the cost of the materials themselves, with the remainder due to cell production and module fabrication [2]. While c-Si has many advantages as a material, including abundancy and manufacturing experience, it is a relatively weak absorber, requiring $200-300 \mu \mathrm{m}$ of semiconductor material to fully absorb the incident sunlight. This c-Si must be high quality and defect free so that the generated carriers are not lost before collection.

Alternative thin-film photovoltaic cells with thicknesses of several microns have been developed with lower processing costs, but also lower efficiencies [3]. In addition to the traditional on-grid markets, thin-film solar cells have been developed on flexible and building integrated platforms. The main thin-film solar technologies at present are cadmium telluride (CdTe), copper indium gallium selenide (CIGS), and thin-film $\mathrm{Si}$, with the record efficiency of these devices at $19.9 \%$ (CIGS) $[4,5]$. Some commercial thin-film solar cell companies have enjoyed tremendous success, particularly those based on CdTe. By early 2010 CdTe had reached production costs of $\$ 0.76 / \mathrm{W}$, with efficiencies around 11\% [6]. Commercial CIGS modules have efficiencies from $9 \%$ to 12\% [6]. However, both CdTe and CIGS cells use scarce elements such as Te and In, and at their current efficiency the technology is not scalable to meet world demand $[7,8,9]$.

Thin-film Si solar cells have the longest commercial history of the thin-film technologies, due to the experience of c-Si technology and the microprocessor industry. These thin-film Si solar cells may be either single junction hydrogenated amorphous Si (a-Si:H) or multijunctions including various other active layers such as microcrystalline $\mathrm{Si}(\mu \mathrm{c}-\mathrm{Si})$ or hydrogenated amorphous silicon germanium (a-SiGe:H). The bandgaps of $\mu \mathrm{c}-\mathrm{Si}$ and a-SiGe:H are sufficiently different from a-Si:H to enable module efficiencies from $8 \%$ to $12 \%$. Commercial single junction a-Si:H solar cells have 


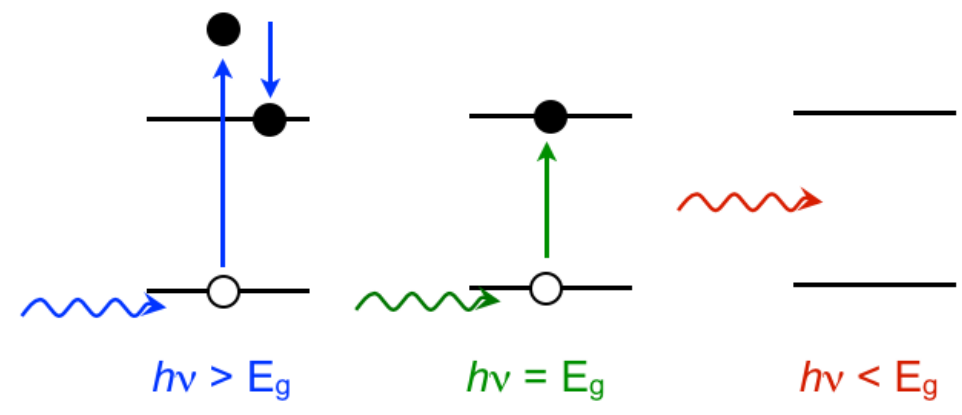

Figure 1.1. Photon energy compared to bandgap of a semiconductor.

slightly lower efficiencies, from $6 \%$ to $7 \%$ [6]. Combined with high capital costs, these lower efficiencies have limited the implementation of thin-film Si modules to date, and improvements in both efficiency and cost are necessary.

\subsection{Solar Cell Fundamentals}

The purpose of a solar cell is to convert incident sunlight to electrical power. When an incident photon hits a solar cell, there are several possible outcomes. It can simply reflect off the surface. As shown in Fig. 1.1, if the energy of the photon is lower than the bandgap of the semiconductor $\left(h \nu<E_{g}\right)$, it can pass through the semiconductor without absorption. If the energy of the photon is higher than the bandgap of the semiconductor $\left(h \nu>E_{g}\right)$ then it can be absorbed by the semiconductor, exciting an electron from the valance band to the conduction band and generating an electron-hole pair (as well as heat, depending on the semiconductor). Solar cells typically include other layers besides the semiconductor, such as contact layers and window layers, which can also parasitically absorb incident sunlight. Efficient solar cells minimize the losses due to reflection and absorption in other materials to maximize absorption in the semiconductor.

After an electron-hole pair is generated, the electron and hole must be separated and driven to collection at separate electrodes. The charge separation occurs at a location of higher electric field. In a standard p-n junction, this occurs by diffusion: the charge carriers diffuse to a p-n junction within the semiconductor and are separated 


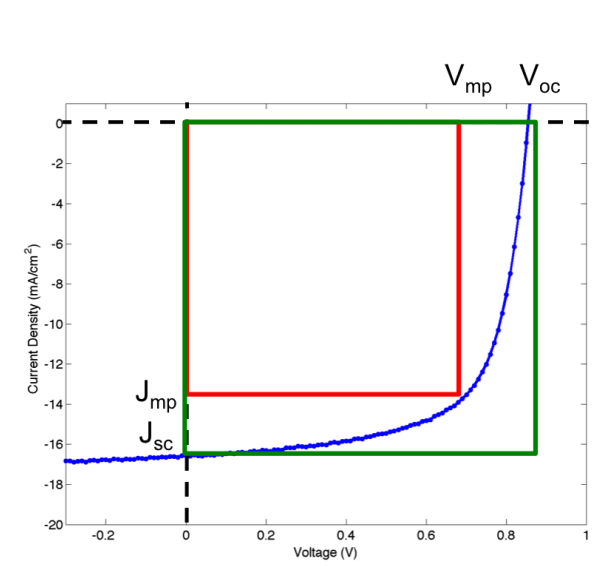

(a)

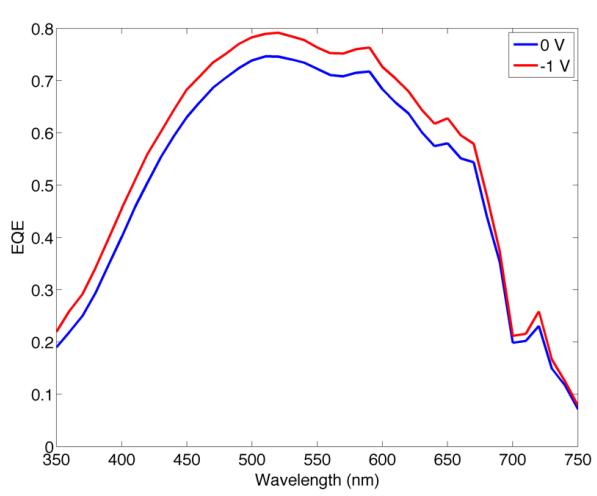

(b)

Figure 1.2. (a) Example J-V curve for a typical solar cell, with the quantities determining efficiency labeled. (b) Example EQE spectrum for an n-i-p a-Si:H solar cell.

by the electric field at a junction. In a p-i-n device architecture, as commonly used in a-Si:H cells, there is an electric field across the entire device which drives the carriers to separation by drift rather than diffusion. Once the carriers are separated they are collected by the electrodes, generating current. Not all photogenerated electrons and holes are collected; other processes such as recombination can prevent the carriers from reaching the electrodes.

There are several basic quantities that can be used to describe the effiiciency of a photovoltaic device. The current voltage (I-V) response of a solar cell can be described by

$$
I=I_{s c}-I_{0}\left(e^{\frac{q V}{k T}}-1\right)
$$

where $I_{s c}$ is the short circuit current, or the current when the voltage of the cell is 0 , and $I_{0}$ is the dark current, or saturation current. Both $I_{s c}$ and $I_{0}$ are dependent on properties such as the materials properties and cell architecture, and the details are described elsewhere [10]. Since the current is proportional to the area of the cell, a more useful quantity than the current $I$ is the current density, $J$, which gives the current per area of the device.

Figure 1.2(a) illustrates a typical J-V curve of a solar cell, with many of the key 
parameters labeled. At low voltages in an ideal solar cell, the current density is close to $J_{\text {sc }}$. As the applied voltage becomes more significant then the current can drop off quickly. At the other extreme of the $\mathrm{J}-\mathrm{V}$ curve, when $J=0$, the cell dissipates its electrical power: this is the open-circuit voltage $\left(\mathrm{V}_{\mathrm{oc}}\right)$. The actual operating point of the cell is at the maximum power point, which determines the cell's efficiency $\eta$.

$$
\eta=\frac{P_{\max }}{P_{i n}}=\frac{F F \cdot J_{s c} \cdot V_{o c}}{P_{i n}}
$$

The fill factor of the device $(F F)$ refers to how close the maximum power point is to the $\mathrm{V}_{\mathrm{oc}}$ and $\mathrm{J}_{\mathrm{sc}}$, and is always less than 1 . The denominator $P_{\text {in }}$ refers to the incident power from sunlight. The $F F$ can be calculated from

$$
F F=\frac{J_{m p} V_{m p}}{J_{s c} V_{o c}}
$$

where $J_{m p}$ and $V_{m p}$ are the current and voltage at the maximum power point, respectively.

Since the measured voltages and currents depend on the illumination, the illumination conditions need to be standardized and specified. One of the most common illumination standards is air mass (AM) 1.5, which corresponds to the suns illumination after traversing an atmospheric thickness of 1.5 atmospheres. The air mass coefficient is the ratio of the path length of light to the path length at the surface normal, and is given by $A M=\frac{1}{\cos z}$, where $z$ is the solar zenith angle. The path length of the sun naturally varies across latitude, time of day, and season, and different conventions are more appropriate for particular locations. For space-based solar cells, the AM0 spectrum is the most relevant. At the equator, AM1 (for the sun directly overhead) is appropriate. Since most solar panels are based at temperate latitudes, the convention is to use the AM1.5 solar spectrum as the standard for comparing cell efficiencies, which corresponds to a solar zenith angle of $48.2^{\circ}$ and has a total power density of $1 \mathrm{~kW} / \mathrm{m}^{2}$.

The other useful figure of merit for photovoltaics is the quantum efficiency of the cell. There are two types of relevant quantum efficiencies: the external quantum effi- 
ciency (EQE) and the internal quantum efficiency (IQE). The EQE is the conversion efficiency of incident photons to collected electrons: ideally every absorbed photon produces one collected electron, but many other processes (such as optical and recombination losses) can make the EQE $<1$. A typical EQE spectrum is shown in Fig. 1.2(b). The IQE is the conversion efficiency of all absorbed electrons to collected electrons, removing optical losses such as reflection and transmission.

Efficient solar cells therefore need to absorb the solar spectrum with minimal losses to reflection or materials other than the semiconductor, and efficiently separate the carriers and collect them externally. This thesis deals primarily with the first challenge: we design nanostructures to absorb light in small volumes of material, and integrate them into photovoltaic devices to study the effects. Carrier collection is not explicitly addressed, but the ultrathin semiconductor volumes used here may improve collection efficiencies as well.

There are two primary challenges to designing solar cells with high absorption: first, the cell must absorb over the entire solar spectrum where the semiconductor is active, which is quite broad, and second the absorption must be high at all angles of incident sunlight. Naturally the angle of incidence changes throughout the day and season, as depicted in Fig. 1.3(a), but the angle will also vary due to diffuse scattering from clouds and other objects (Fig. 1.3(b)). While tracking devices can be used to optimize the angle of incidence of the sun, it is nevertheless important for solar panels to operate well at a wide range of incident angles.

\subsection{Ultrathin-film Solar Cells}

One approach to next-generation solar cell development is to use ultrathin-film absorbing layers. In ultrathin-film solar cells, light trapping to improve the current is critical to preserve high efficiencies. Thinner layers naturally require fewer raw materials, making the cells less expensive. Given that for c-Si technologies, however, the cost of the material is approximately $50 \%$ of the cost of the total module fabrication cost, these cost reductions will be significant but not game-changing. Ultrathin-film 


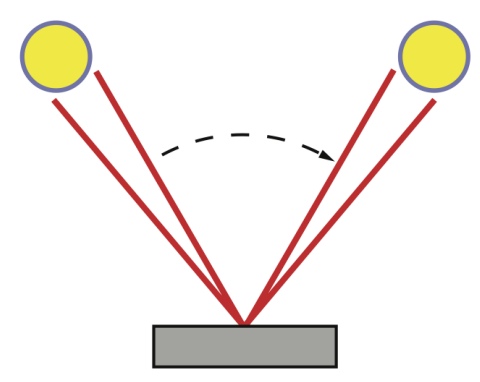

(a) Direct angle of incidence.

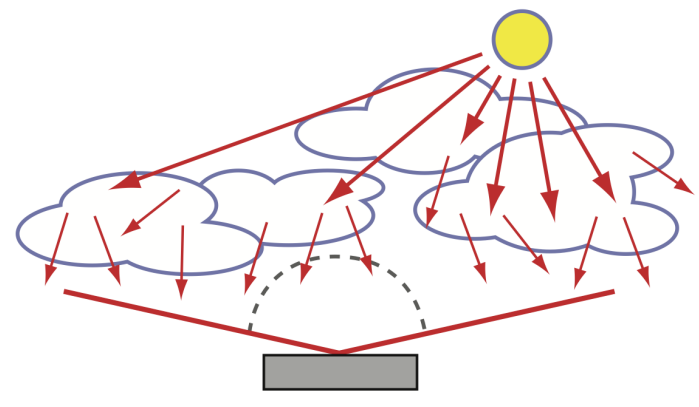

(b) Diffuse angle of incidence.

Figure 1.3. Schematics of direct and diffuse angles of incident sunlight striking a solar cell.

solar cells must then have other advantages over their thin-film counterparts to be beneficial.

\subsubsection{Availability of Raw Materials}

Many of the materials used in current thin-film photovoltaic technologies, such as $\mathrm{CdTe}$ and CIGS, include elements that are among the rarest materials on earth. Tellurium, for example, is one of the nine rarest metals on earth, with concentrations between 1 and 5 parts per billion in the crust. Since these elements are scarce they are usually isolated as by-products of mineral purification for other more common elements; for example, Se and Te are produced largely from $\mathrm{Cu}$ mining, and In is produced from Zn mining [7]. As demand increases for these elements in technological applications, such as the use of In for digital displays, the market prices for these rare elements may rise.

The reduction of feedstock materials can help circumvent problems associated with the limited abundance of materials desirable for solar cells. Several studies have examined the material abundance limits for the most common thin-film technologies $[8,9]$. The estimates discussed here show how improved light trapping (and a concomitant reduction in cell thickness) modifies the material abundance constraints on scalability. We consider single junction cells without additional layers (such as window layers), with all of the material utilized for energy production. Since the costs of 


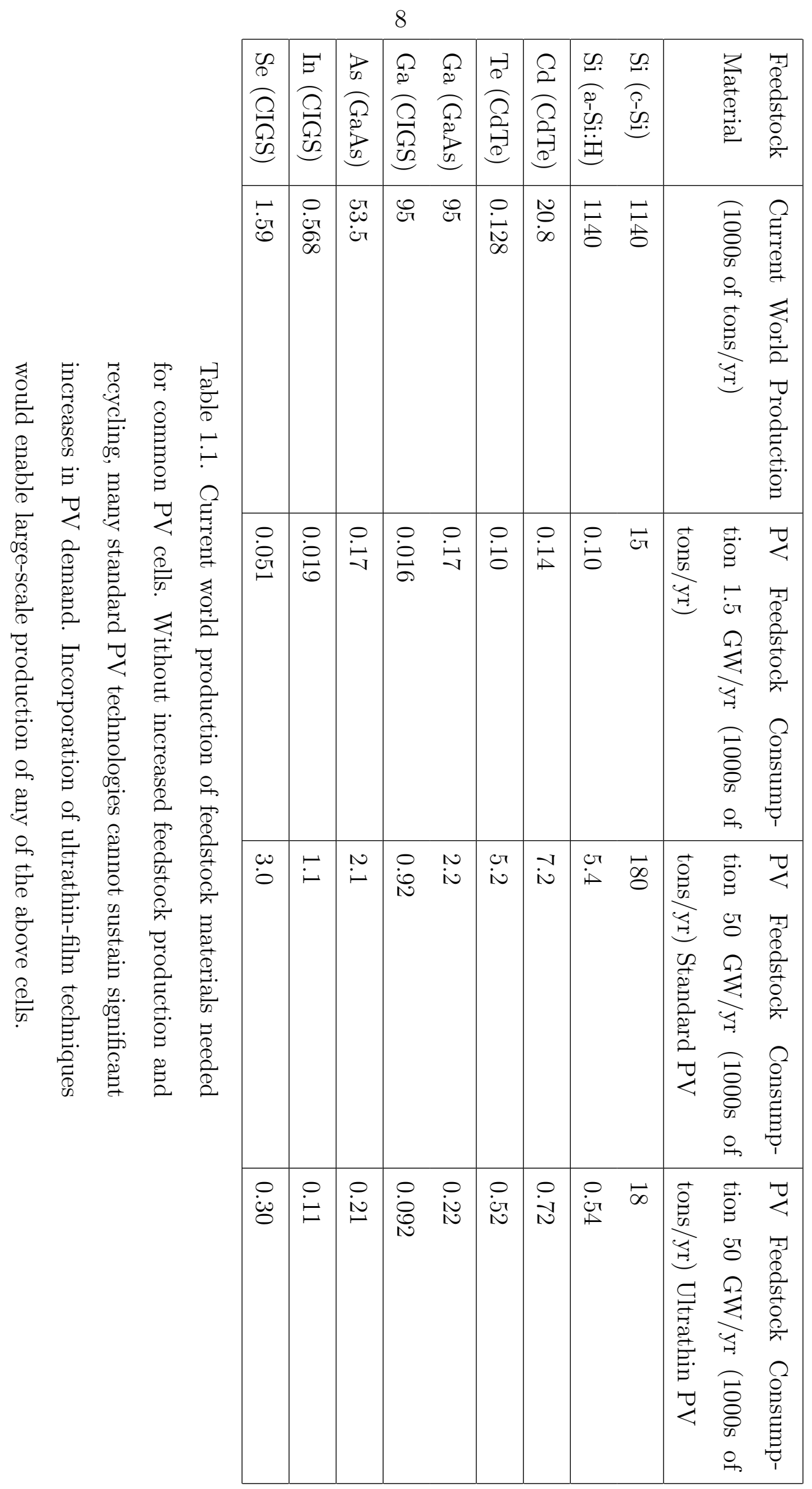


extraction can vary due to demand, we consider only the abundance of the material rather than the cost of mining and purification. We also do not consider economic reserves, only annual production.

Currently, PV cells make up a very small fraction of the worlds total energy portfolio $(<1 \%)$; however, as the demand for renewable energy increases, PV can expect marked growth. We calculate a mass/power for each of the cells listed in Table 1.1 under different light trapping scenarios, and use this to estimate the amount of material necessary to meet different energy demands. We estimate a thickness under standard light absorption for each cell by setting the thickness to approximate current production values (200 $\mu \mathrm{m}$ for c-Si and $3 \mu \mathrm{m}$ for all other materials). The mass/power $(\mathrm{m} / \mathrm{P})$ ratio for each element is given by

$$
\frac{m}{P}=\frac{t \rho}{I A \eta}
$$

where $t$ is the thickness of the cell, $\rho$ is the density of the element, $I$ is the incident flux $\left(1000 \mathrm{~W} / \mathrm{m}^{2}\right), A$ the cell area, and $\eta$ the cell efficiency. For simplicity, we assume that the efficiencies remain near that of current production modules.

As PV power comes into production, the first modules will require materials entirely from feedstock consumption. After year two, however, the lifetime of the cells and recycling of materials becomes important. The scenario outlined here looks only at the initial production and does not consider recycling. The second column of Table 1.1 shows current world production of the various PV feedstock materials based mainly on USGS estimates. The third column shows the feedstock consumption to produce $1.5 \mathrm{GW} /$ year, approximately the amount of PV power produced by the US in 2005. In the fourth section of Table 1.1 we project the amounts of material required to reach $50 \mathrm{GW} /$ year production of new PV. Under the same thickness requirements, there are feedstock limitations to Te, In, and Se. Improved light trapping that reduces the thickness of the cell to one tenth its previous value (with the same efficiency) removes the limitations for In and Se, although Te production is still an issue. 


\subsubsection{Throughput}

For thin-film Si photovoltaics, materials abundance is not a substantial difficulty. Relative to the other thin-film technologies, however, thin-film Si modules have been less successful, due in part to capital-intensive fabrication [6]. Commercially available thin-film Si cells (both single junction and multijunction) rely on plasma-enhanced chemical vapor deposition (PECVD) for deposition of the Si layer. PECVD produces high quality layers but suffers from low deposition rates $(0.3-0.5 \mathrm{~nm} / \mathrm{sec})$, although improving both the rate of PECVD and the quality of other CVD processes has been an intense focus of research [11]. Commercial thin-film Si production compensates for this deposition rate by either increasing the length of the deposition line, or using many cluster systems in parallel, which drives up the capital cost [12]. Ultrathin-film solar cells, however, require much shorter deposition times as the layer thickness is reduced, directly improving fabrication throughput and reducing the cost. A reduction thickness by a factor of 2 would greatly improve manufacturability of Si PV modules..

\subsubsection{Carrier Collection}

Most collection-limited materials would benefit from ultrathin-film devices with optically thick absorption. Materials such as organic polymers, quantum dots, and polycrystalline or amorphous inorganic semiconductors could all benefit from reduced layer thicknesses, particularly if the thickness can be reduced to be comparable to the minority carrier diffusion length. Many cells made from these materials face a tradeoff between complete light absorption and carrier collection, limiting the achieved efficiencies. Alternative device architectures have also been utilized for these materials, including p-i-n drift based devices for thin-film Si and bulk heterojunctions for organic cells, although these devices still have some restrictions on the thickness for complete carrier collection.

\subsubsection{Improvement in $\mathrm{V}_{\mathrm{oc}}$}

The open-circuit voltage of a cell can be expressed as [13] 


$$
\mathrm{V}_{\mathrm{oc}}=\frac{k T}{q} \ln \left[\frac{J_{L}}{J_{0}}+1\right]
$$

where $J_{L}$ is the current density under light illumination (or the photocurrent), and $J_{0}$ is the dark current density, or the saturation current density. The dark current depends proportionally on the volume of material, and so decreasing the thickness can also decrease the dark current. If the photocurrent $J_{L}$ can remain high in an ultrathin solar cell due to light trapping, then it is possible for an ultrathin device to be more efficient than a thicker device due to the increased $\mathrm{V}_{\mathrm{oc}}$. However, one must also consider the reduced shunt resistance and surface recombination effects in ultrathin layers: this increase in $\mathrm{V}_{o c}$ will not be realized in all devices. Chapter 6 discusses this effect in more detail.

\subsubsection{Stability}

The main disadvantage of a-Si:H solar cell technology is the decline in efficiency under the first several hours of light illumination, the well-known Staebler-Wronski effect [14]. All a-Si:H solar cells exhibit this light-induced metastability, which primarily degrades the fill factor and substantially decreases the efficiency. Commercial a-Si:H modules typically degrade by $10 \%$ to $30 \%$ from their initial values under operation, but the degradation can be reversed by annealing. Reducing the thickness of the intrinsic layer in a p-i-n or n-i-p a-Si:H device can mitigate the degradation: the higher electric field over the intrinsic layer appears to reduce the effect $[11,15]$. The increased stability of thin a-Si:H layers has led to the development of multijunction Si solar cells where intentionally thinned a-Si:H is stacked with other $\mathrm{Si}$ materials. Chapters 6 - 8 consider single-junction a-Si:H solar cells with ultrathin intrinsic layers, with the aim of increasing absorption to produce high efficiency, stable solar cells.

\subsubsection{Absorption and Layer Thickness}

While there are many cost and performance advantages of ultrathin-film solar cells, efficient devices have not been realized due to the reduced absorption. Naturally, 


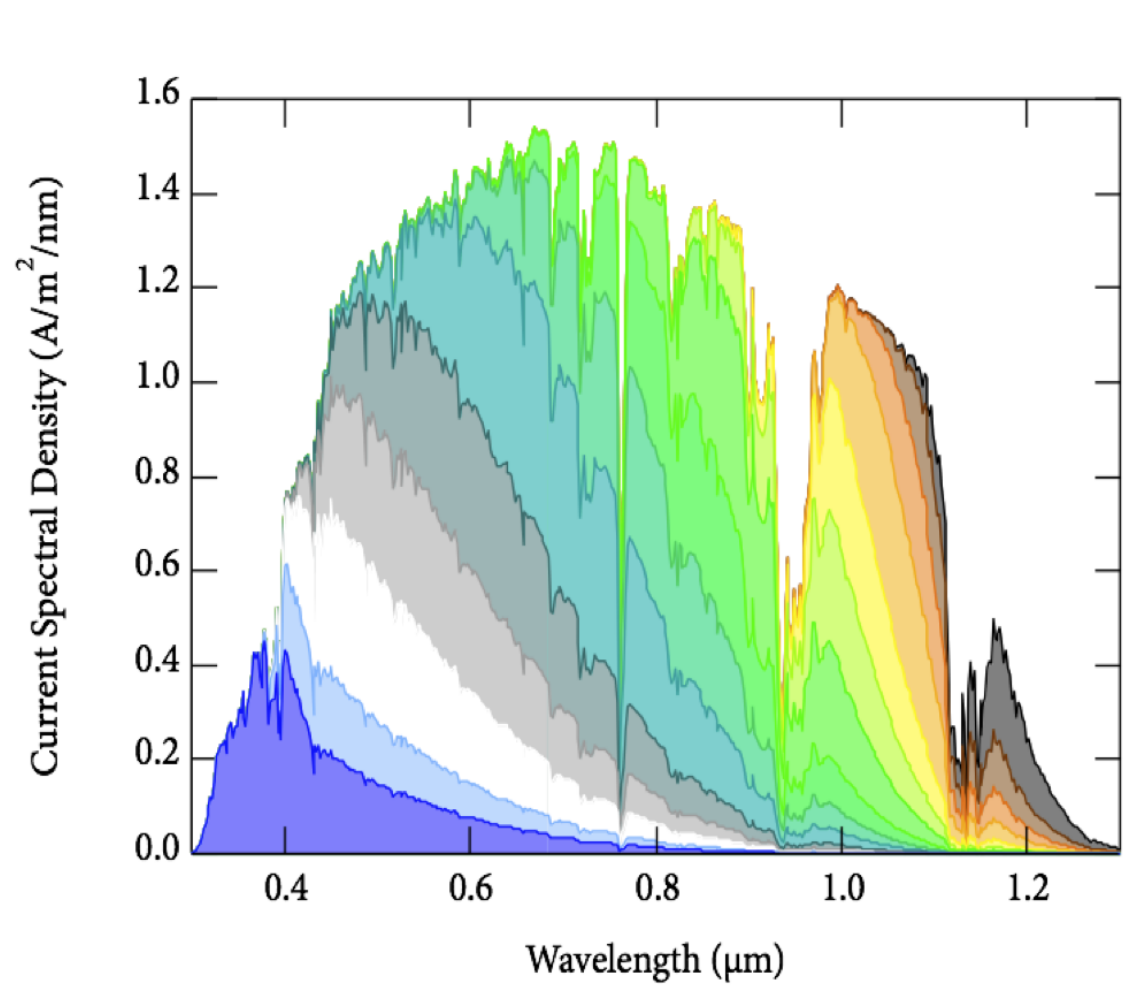

$h(\mathrm{Si})$

$\square 100 \mathrm{~nm}$

$200 \mathrm{~nm}$ $500 \mathrm{~nm}$

$1 \mu \mathrm{m}$

$2 \mu \mathrm{m}$

$5 \mu \mathrm{m}$

$10 \mu \mathrm{m}$

- $20 \mu \mathrm{m}$

- $50 \mu \mathrm{m}$

$100 \mu \mathrm{m}$

$200 \mu \mathrm{m}$

- $500 \mu \mathrm{m}$

$\square 1000 \mu \mathrm{m}$

$\square 2000 \mu \mathrm{m}$

$\square 5000 \mu \mathrm{m}$

AM1.5 Spectrum $1 \mathrm{~kW} / \mathrm{m}^{2}$

Figure 1.4. Fraction of the AM1.5 solar spectrum absorbed in a single pass through c-Si layers of varying thickness. 
13

as the thickness of the semiconductor is reduced the absorption decreases. Figure 1.4 shows the AM1.5 solar spectrum and the fraction of its power absorbed at each wavelength in a single pass through c-Si of varying thickness. As the thickness of the c-Si is reduced, there is a significant decrease in absorption, particularly in the portion of the spectrum from $\lambda=600 \mathrm{~nm}$ to $1100 \mathrm{~nm}$. This weak absorption is the reason standard c-Si solar cells are 180 - $300 \mu \mathrm{m}$ thick. For ultrathin c-Si, substantial improvements will be necessary across the entire spectral range where c-Si is absorbing. To realize all the advantages described above for ultrathin-film solar cells, broadband light trapping structures are necessary to achieve high photocurrents in small semiconductor volumes.

\subsection{Approaches to Light Trapping}

Light trapping, where the surface of the cell is structured or textured to confine light within the semiconductor, allows for absorption over multiple passes. This allows for the quantity of material to be reduced, since not all absorption occurs in a single pass. Light trapping is a major component of many commercial solar cells: c-Si cells often include pyramidal surface texturing to reflect light from the surfaces into the c-Si layer, and a-Si:H solar cells employ random surface textures to increase light absorption. The considerations for thick and thin-film light trapping designs are quite different: for thick films light trapping can be described using ray optics, while thin-films require treatment with wave optics.

\subsubsection{Ergodic Limit}

In thick films, an ideal light trapping surface will exhibit "ergodic" behavior if the cell texturing is sufficient to fully randomize the direction of light within the cell. The refractive index contrast between the semiconductor and the surrounding medium (i.e., air) results in total internal reflection, which in turn increases the path length of the photon within the semiconductor and therefore the absorption. Some light may still exit the semiconductor from an escape cone of angle $\theta$. Yablonovitch has shown 

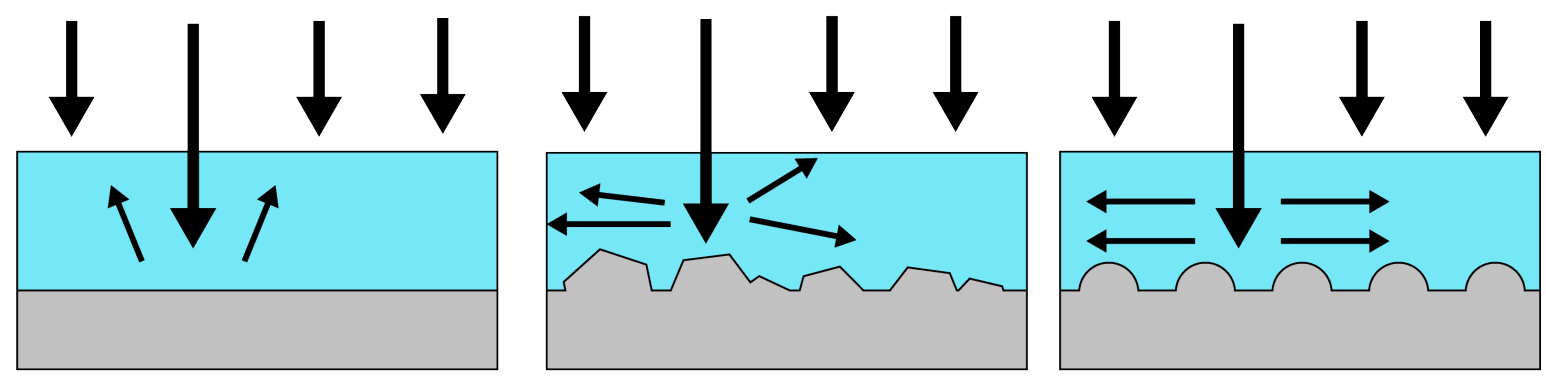

Figure 1.5. Approaches to light trapping in thin-films.

that the effective path length can increase by up to a factor of $2 n^{2} / \sin \theta$, where $n$ is the refractive index, or $4 n^{2} / \sin \theta$ including a back reflector [16]. This limit will only be reached near the band edge, where absorption in the semiconductor is weak. For c-Si with $n \approx 3.5$, this translates to a path length enhancement of $50 \times$ including the back reflector.

\subsubsection{Thin-films}

For smooth thin-films, absorption is determined by reflection and refraction off the interfaces, the material and its thickness, and the coherence of the incident light (left panel of Fig. 1.5). If the light source is coherent over the thickness of the film, then it may act as a Fabry-Perot cavity where some wavelengths will be resonantly absorbed [17]. The reflection and transmission off of each interface at normal incidence can be calculated using Fresnel's coefficients, with the phasors tracked and summed over multiple passes. This treatment will be applied in Chapter 4. It can also be extended to off-normal angles of incidence via transfer matrix methods, or solved numerically.

Many thin-film devices also employ random surface texturing, as shown in the middle of Fig. 1.5. For a-Si:H solar cells, these light trapping surfaces are often introduced through a textured substrate or superstrate, depending on the order of deposition. A wide variety of light trapping random structures have been used, which either develop native texture during deposition or are etched after deposition. Much of the optimization of these surfaces is heuristic: deposition or etch parameters are varied, and cells incorporating these textures are measured and compared. When 
the scattering of the surface is measured or calculated, the standard parameter is the haze.

$$
\text { haze }=\lim _{r \rightarrow \infty}\left[\frac{\int_{0}^{\pi} \int_{0}^{2 \pi} S_{r}(r, \theta, \phi) d \theta d \phi-S_{r}(r, 0,0)}{\int_{0}^{\pi} \int_{0}^{2 \pi} S_{r}(r, \theta, \phi) d \theta d \phi}\right]
$$

Here $S_{r}$ is the radial component of the Poynting vector, and $\theta$ and $\phi$ are the spherical coordinates. The haze describes the amount of non-specularly scattered light by the substrate. However, haze is typically described and measured for the substrate against air, which does not accurately reproduce the scattering behavior in a solar cell where it is in contact with a different index material, and so the connection to absorption enhancement is unclear. Other scattering models utilize a variety of computational and semi-analytic techniques to connect to other metrics of scattering from rough surfaces. We treat the problem numerically, and show in Chapter 7 that our calculations of absorption enhancement in randomly textured solar cells compare well to experimental measurements. Chapter 8 describes the role of randomness in light trapping design in more detail, and includes the design, fabrication, and modeling of pseudo-random structures with optimized spatial frequencies.

The third alternative is to design nanophotonic structures to efficiently trap light in thin volumes. Over the time frame of this thesis, light trapping with nanophotonic structures has increased in popularity, with the demonstration of solar cells including nanophotonic structures such as nanowires, nanocones, photonic crystals, coaxial structures, nanoparticles, and gratings $[18,19,20,21,22,23,24,25,26,27,28$, 29]. Recent papers have also sought to quantify the upper limit to light trapping in the wave optics limit; Chapter 5 describes one method for comparing nanophotonic structures to the ray-optics ergodic limit $[30,31,32]$. This thesis as a whole aims to couple incident light into guided modes of a thin or ultrathin semiconductor volume, as depicted in the right panel of Fig. 1.5. The light is coupled into a guided mode which propagates in the plane of the solar cell, while carrier collection occurs in the out-of-plane direction. This allows for the use of ultrathin absorbing layers without sacrificing absorption. 


\subsection{Plasmonics}

The unusual optical properties of surface plasmons, the coherent oscillation of electrons on the surface of a metal, have been studied for a number of years. The most famous historical example is the Lycurgus cup from the 4th century AD, which exhibits different colors under reflection and transmission. This unusual optical property was obtained by mixing subwavelength $\mathrm{Ag}$ and $\mathrm{Au}$ nanoparticles into the glass which scatter green light but transmit red light, resulting in different colors. Quantitative studies of the optical properties of metal nanoparticles began in earnest near the beginning of the 20th century, and were particularly bolstered by Gustav Mie's seminal 1908 paper on the scattering of light by spherical objects, including by $\mathrm{Au}$ [33]. In 1957, Ritchie predicted the existence of surface plasmons, and by 1968 Otto, Kretschmann, and Raether had shown that surface plasmons could be excited optically $[34,35,36]$.

One of the first major application areas of surface plasmons to emerge was in molecular sensing: rough metallic films were shown to enhance the Raman signal of molecules by several orders of magnitude, and the effect was explained via electromagnetic interactions $[37,38]$. With advances in nanofabrication, a wide variety of

other applications of surface plasmons have emerged in recent years, including optoelectronics components, enhancement of local emitters, materials with negative index of refraction, imaging below the diffraction limit, light emitting diodes, plasmonic lasers, and cancer therapy [39, 40, 41, 42, 43, 44, 45].

While surface plasmons in general exist on any interface of a metal, most research has focused on either localized surface plasmons, such as those on the surface of a nanoparticle, or planar surface plasmons characterized by propagating charge compression waves on the surface. In both cases the wavelengths are small and the electric field intensities high compared to free space. 


\subsection{Plasmonic Photovoltaics}

Inspired by the techniques of optoelectronics, surface plasmons have recently attracted attention for use in solar cells. Indeed, an ultrathin-film solar cell can be considered as an optical integrated circuit, in which light is received, guided, localized, and collected at the nanoscale. The large resonant scattering cross sections of metal nanostructures offer the potential to scatter light strongly, while surface plasmon polaritons can guide and confine light in nanoscale dimensions. The main mechanisms for absorption enhancement in photovoltaics via surface plasmons will be discussed in detail in Chapter 2, but a brief historical perspective will be provided here.

The field of plasmonic photovoltaics has emerged in force over the time scale of this thesis, with the number of literature papers and interested commercial manufacturers expanding rapidly, particularly from 2008 onward. The field has benefited greatly from both improved large-area nanofabrication techniques and electromagnetic calculation, which are both discussed throughout this thesis. To date plasmon-enhanced photocurrent in photovoltaics has been demonstrated in a wide variety of solar cells, including those based on c-Si, a-Si:H, GaAs, CdSe, InGaN/GaN, InP/InGaAsP, organic semiconductors such as polythiophene and copper pthalocyanine, and hybrid organic-inorganic devices such as dye sensitized solar cells $[46,47,48,49,50,51,52$, $53,54]$. In parallel, several groups have published theoretical and simulation results on plasmonic solar cell designs.

The most common plasmon enhanced solar cell structure to date consists of metallic nanoparticle scatterers (of various shapes) deposited on top of an already fabricated solar cell. These cells have the advantage that the fabrication process does not have to be substantially modified to incorporate plasmonic scatterers. The disadvantage is that, since the particles are on top, attention must be paid to the density of objects to avoid shadowing. The presence of particles on top also modifies the use of AR coatings, and so any added benefits from nanoparticles must be weighed against the benefits due to conventional antireflection methods $[55,56]$.

The first papers to explore the potential of surface plasmons in photovoltaics were 
published in the late 1990s by Stuart and Hall [57, 58, 59]. Their device consisted of a thin silicon-on-insulator (SOI) wafer, where the Si was $165 \mathrm{~nm}$ thick, and since the absorbing $\mathrm{Si}$ layer was separated from the Si substrate by a layer of $\mathrm{SiO}_{2}$, it acts as a thin waveguide. Metal island formation was achieved by depositing a thin metallic film and annealing under $\mathrm{N}_{2}$ so that the film coalesced into discrete islands. The size and shape of the particles could be tuned to some extent by varying the thickness of the metal layer and the annealing time and temperature, although the patterns and spacings were irregular. The authors report a photocurrent enhancement as high as 18 at $800 \mathrm{~nm}$ with the addition of Ag islands. Since then other groups have extended the island annealing technique on $\mathrm{Si}$ and other absorbers. Similar results were noted in 2007, where a photocurrent enhancement of 16 for a $1.25 \mu \mathrm{m}$ SOI cell due to $\mathrm{Ag}$ nanoparticles was reported, with a solar spectrum integrated enhancement of $30 \%$ $[46]$.

A second fabrication approach uses colloidal Ag and Au nanoparticles as the source of scattering objects. This allows for uniformity of the size and control of the density of particles, although it is difficult to pattern the particles precisely and prevent aggregation. This type of deposition has been used on crystalline $\mathrm{Si}$ (c-Si), a-Si:H, and an InP/InGaAsP quantum well p-i-n structure [51, 60, 61]. Other deposition techniques include island formation on TCO's for organic photovoltaics, incorporation of metal nanoparticles inside the active layer, electron beam lithography, and electrodeposition $[62,63,64,65,66]$.

Within the Atwater group others utilized a fabrication technique based on the use of anodic aluminum oxide (AAO) templates as evaporation masks for the deposition of metallic nanoparticles $[48,50]$. The AAO templates can be used to controllably and reproducibly deposit metallic particles onto a cell via evaporation through the template. The hole density of the template is controlled by the applied voltage during anodization, the hole diameter is controlled by the etch time, and the particle height is controlled by the metal deposition thickness during evaporation [66]. Upon annealing, hemispherical and conical particles can be formed. For optically thin p-n photovoltaic layers of GaAs (200 nm), that do not support waveguide modes due to an index- 
matched absorbing substrate, an $8 \%$ increase in $\mathrm{J}_{\mathrm{sc}}$ was observed for $\mathrm{Ag}$ nanoparticles. For a single InGaN quantum well with plasmonic scatterers, experiments showed a $54 \%$ increase in EQE and a $6 \%$ increase in $\mathrm{J}_{\mathrm{sc}}$.

The experimental portions of this thesis utilize Substrate Conformal Imprint Lithography (SCIL), a nanoimprint method developed by Philips Research. The process allows for patterning over 6 - 8 " wafers with nanoscale structures and spacings. The process will be described in Chapter 6, and experimental measurements of cells patterned with SCIL are discussed in Chapters 6 and 8.

\subsection{Overview of Thesis}

This thesis describes the design, modeling, fabrication, and measurement of solar cells incorporating plasmonic nanostructures for enhanced photocurrent. In particular, we focus on plasmonic nanostructures built into the metallic back contact of a thin-film solar cell, although other geometries will be discussed as appropriate. Incident sunlight scatters from the nanostructures on the back contact and couples to waveguide modes of the solar cell, and the power in the mode is absorbed as it propagates. Chapter 2 provides an overview of the mechanisms for plasmonic enhancement of absorption in photovoltaics, along with a description of the waveguide modes and scattering cross sections from metallic nanostructures. Chapter 3 then describes the development of a numerical toolbox for use in understanding these structures. We develop a technique for numerically identifying the modes in a waveguide and the incoupling efficiency from sunlight to particular modes, as well as the direct calculation of absorption in a nanophotonic solar cell. In Chapter 4 we apply this method to understand the factors that affect coupling from isolated nanostructures to waveguide modes across the solar spectrum. Chapter 5 then studies the modes in more detail, calculating which modes have the most potential for strong absorption in the semiconductor, and analyzing the potential for absorption enhancement due to waveguide modes under idealized limits.

The second part of this thesis explores the integration of plasmonic nanostructures 
in experimental solar cells, focusing particularly on a-Si:H. Chapter 6 shows the use of the SCIL method for nanopatterning large areas, and that plasmonic nanostructures can be designed which show increased photocurrent over cells with randomly textured light trapping surfaces. We also experimentally verify that coupling to waveguide modes enhances the photocurrent. In Chapter 7, we show that electromagnetic simulation can accurately reproduce absorption from experimental cells with both designed and random texture, and use this to understand and optimize the mechanisms for light trapping across the spectrum. The final chapter shows the fabrication of high efficiency, ultrathin-film solar cells with angle-independent photocurrent, and proposes a general theory for the design of broadband light trapping surfaces. The ultimate device consists of integrated plasmonic scatterers on the back metal contact with resonantly absorbing semiconductor nanostructures on the front surface, creating a broadband photocurrent enhancement due to a combination of effects across the solar spectrum.

Over the last three years, plasmonics has emerged as a promising area of research for enhanced performance in photovoltaics, and is now included on road maps with other third-generation concepts. While this thesis shows development of one design from theory to integrated, high-efficiency devices, the promise of plasmonics in photovoltaics is still emerging, and the future may hold many more advances. 


\section{Chapter 2}

\section{Plasmonic Solar Cells}

\section{$2.1 \quad$ Introduction}

This chapter describes the optical properties of localized and propagating surface plasmons, as applied to photovoltaics. Metals are traditionally seen as recombination centers or sources of optical losses in photovoltaics, and the idea of using metal nanostructures to enhance absorption in solar cells is at first counterintuitive. The unusual properties of plasmonic nanostructures, however, including their scattering cross sections, high local electric fields, and propagating waveguide modes, enable their use in solar cells. The first section of this chapter describes the resonant scattering cross sections of small metal nanoparticles, and considerations when these scatterers are introduced in solar cells. The second part describes the waveguide modes that can be excited in a solar cell, both photonic slab waveguide modes and SPP modes. The challenge for photovoltaic applications is to design scattering objects with high effective cross sections that are both broadband across the solar spectrum and insensitive to angle of incidence.

\subsection{Overview of Mechanisms for Plasmonic Ab- sorption Enhancement}

There are a few distinct plasmonic mechanisms for enhancing absorption in solar cells,

which together encompass most of the explored devices to date. First, subwavelength metal nanostructures (on either the front or back of an absorbing layer) can be used 

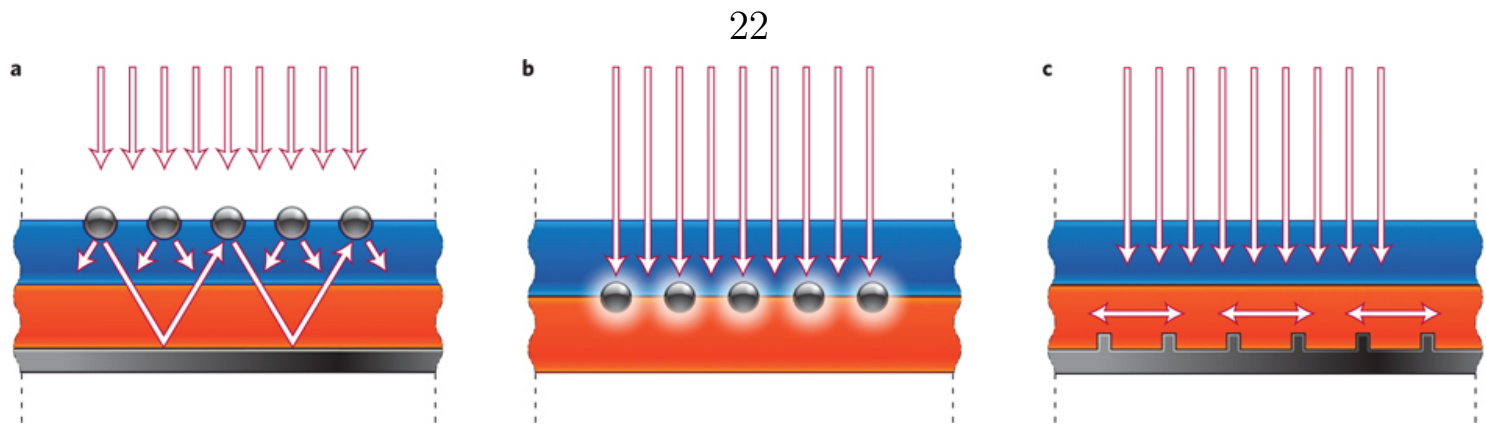

Figure 2.1. (a) Light trapping by nanoparticles from metal nanoparticles on the top surface of the solar cell. Light is scattered and trapped into the substrate at a higher angle, increasing the path lenght. (b) Light absorption enhancement in the semiconductor by embedded nanoparticles due to the enhanced near field of the nanoparticles. (c) Light trapping by coupling to guided modes of the solar cell from nanopatterned metallic back contacts.

to scatter incident light into a distribution of angles, increasing the path length of the light within the absorbing layer (Fig. 2.1(a)). This can lead to higher short circuit current densities, in both relatively thick cells and thin cells on index-matched substrates $[46,48]$.

A second plasmonic mechanism for enhanced absorption results from high nearfield intensities associated with the localized surface plasmonic resonance of the particle (Fig. 2.1(b)). Depending on the shape and size of a metallic particle, particular frequencies of optical excitation will result in strongly enhanced fields near the particle. Since the optical absorption is proportional to the field intensities, high local fields lead to increased absorption. This can be a challenging geometry, however, as the embedded metal particles can lead to semiconductor defects or act as recombination centers, in addition to the fabrication challenges. Most of the successful solar cells of this geometry are polymer devices, where metal particles can be incorporated through spin-casting [62].

The third mechanism uses the scattering center as a means to excite propagating waveguide modes within the thin absorbing layer (Fig. 2.1(c)). The propagating waveguide modes may be either photonic waveguide modes or surface plasmon 


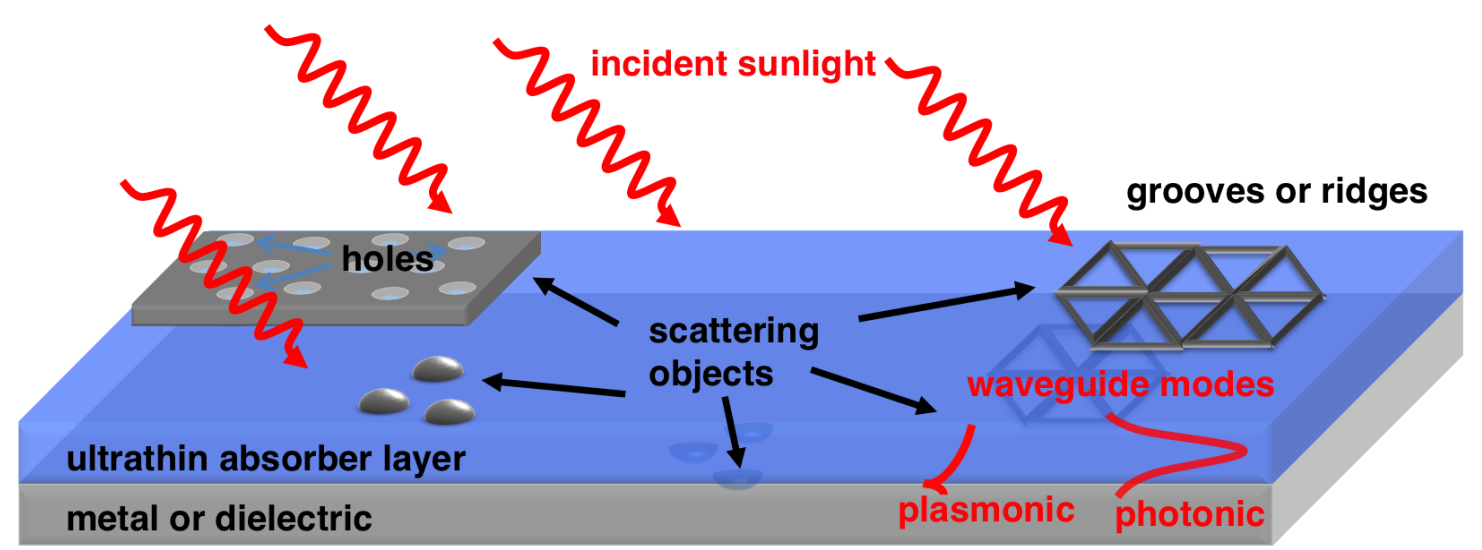

Figure 2.2. Generalized scatterers for coupling into waveguide modes in a solar cell, showing some of the potential variables for designing plasmonic nanostructures. Scatterers can consist of particles on top, middle, or back of the solar cell and could contain layers of metal, dielectrics, transparent conducting oxides, or air. Incident sunlight is scattered into photonic or SPP modes depending on the scattering object and incident wavelength of light.

polariton (SPP) modes, and the scattering center is used to overcome the momentum mismatch between the incident wavevector and that of the waveguide mode $[32,57,67,68]$. As the mode propagates, the power in the waveguide mode will be absorbed partially in the semiconductor layer, thereby exciting electron-hole pairs and enhancing absorption by redirecting the light horizontally. Carrier collection usually occurs in the vertical direction, orthogonal to the absorption path. This third design is the focus of most of this thesis.

Figure 2.2 illustrates several possible architectures for integrating scattering objects into photovoltaic devices. Variables include the shape of the scattering center, its relative height in the waveguide, arrangement with respect to other scattering objects, and the use of either dielectric or metallic surrounding layers. For example, the scattering objects may be nanoparticles, gratings, or holes in an otherwise continuous metal film. The shape of the nanostructure is another variable: hemispheres, cylinders, cones, or anisotropic shapes are all possibilities, each with their own scattering 
cross section and angular scattering distribution [69]. The scattering from different shapes of nanostructures is discussed in Chapter 4. We show one potential tiling of grooves or ridges, a hexagonal arrangement for efficient and polarization-independent surface coverage. Of course the scattering objects could be tiled in square lattices or more complicated patterns, or arranged in a quasi-random geometry, as discussed in Chapters 6 - 8. Dielectric spacer or passivation layers between the semiconductor and the metal can be used to tune the spectral response of the scatterers. This is the case in the experimental devices discussed in Chapters 6 - 8 , and the influence of the spacer layer is specifically discussed in Chapter 7. In summary, the optimization of the shapes and arrangements of scatterers for broadband, large area absorption enhancement represents a significant platform for understanding the magnitude and limits to plasmonic photovoltaic designs.

\subsection{Plasmonic Scattering}

The introduction of scattering objects into a solar cell modifies the standard exponential absorption profile to create spatially inhomogeneous absorption. The intensity at a given depth in a cell with a planar surface is related to the incident intensity $I_{\text {in }}$ by $I=I_{\text {in }} e^{-a L} ; L$ is the path length of the light in the medium, and $a$ is the absorption coefficient of the material. The simplest architecture for a solar cell employing scattering objects is a conventional, thick device with a sparse array of subwavelength metal scatterers deposited on the top, where incident light scatters independently at each scatterer and collective scattering modes are neglected [70]. We assume minimal transmission $T(\lambda)$, so that the power is either absorbed (in the semiconductor, metal, or supporting layers) or reflected (including backscattering). In the absence of interference between the scattering objects, the fraction of total power absorbed in the semiconductor $A(\lambda)$ is

$$
A(\lambda)=\xi Q_{\text {Scat }}(\lambda) f_{\text {substrate }}(\lambda)+\left(1-\xi Q_{\text {scat }}(\lambda)\right)\left(1-R_{s}(\lambda)\right)
$$

where $\xi$ is the fraction of the surface covered by scatterers, $Q_{\text {scat }}$ is the normalized 
scattering cross section of the nanoparticle relative to its physical size, $f_{\text {substrate }}$ is the fraction of the total scattered light that is forwardscattered into the substrate, and $R_{s}(\lambda)$ is reflection from the semiconductor interface only, in the absence of a nanoparticle. For normally incident light the path length of the scattered light is increased to $\frac{L}{\cos (\theta)}$, where $\theta$ is the angle between the scattered light and the surface normal. The reflection term $R_{s}(\lambda)$ is reflection from the semiconductor interface only, in the absence of a nanoparticle.

The normalized quantity $Q_{\text {scat }}$ can be calculated from the scattering cross section $\sigma_{\text {scat }}$ using $Q_{\text {scat }}=\frac{\sigma_{\text {scat }}}{\sigma_{\text {geom }}}$, where $\sigma_{\text {geom }}$ is the geometrical cross section of the object. In the quasistatic limit where the nanoparticles are much smaller than the wavelength of incident light and the field on the particle is uniform, the scattering cross section is given by

$$
\sigma_{\text {scat }}=\frac{1}{6 \pi}\left(\frac{2 \pi}{\lambda}\right)^{4}|\alpha|^{2}
$$

where $\alpha$ is the polarizability of the particle [71]. For spherical nanoparticles embedded in a semiconductor with permittivity $\epsilon_{s}$, the polarizability is calculated from

$$
\alpha=4 \pi r^{3} \frac{\epsilon_{m}-\epsilon_{s}}{\epsilon_{m}+2 \epsilon_{s}}
$$

where $r$ is the radius of the nanoparticle and $\epsilon_{m}$ is the permittivity of the metal. When $\epsilon_{m} \approx-2 \epsilon_{s}$, as occurs in the noble metals in the visible region of the spectrum, the polarizability is at a maximum and the particle exhibits a dipolar surface plasmon resonance. The quantity $Q_{\text {scat }}$ thus depends on both the size of the particle and the local environment, both of which can be used to tune the scattering properties. The absorption cross section $\sigma_{a b s}$ in the metal scales with the polarizability.

$$
\sigma_{a b s}=\frac{2 \pi}{\lambda} \Im[\alpha]
$$

For solar cell applications an important design criterion is that $\sigma_{\text {scat }}>>\sigma_{a b s}$ to keep metallic losses low, since sunlight absorbed in the metal will not contribute to useful carrier generation. In the case of the smallest nanoparticles, $\sigma_{s c a t}+\sigma_{a b s} \approx \sigma_{a b s}$ 
but as the particle size increases $\sigma_{\text {scat }}$ grows and the dipolar plasmon resonance red shifts and broadens. As the size of the nanoparticle approaches $\lambda$, both the quasistatic approximation breaks down and multipolar modes contribute to the particle's scattering cross section. All of the equations given above are valid for dielectric particles as well as metallic ones and the difference lies in $\epsilon_{m}$ : dielectric particles have both more modest permittivities than metals and $\Re\left[\epsilon_{d}\right]>0$, and thus do not exhibit resonant behavior.

This resonant dipolar scattering behavior is not limited to spherical metal nanoparticles; metallic voids exhibit similar behavior, and a wide variety of other nanoshapes can be made including cylinders, hemispheres, core-shell particles, and anisotropic particles. Each different shape of particle will have a different $f_{\text {substrate, }}$, so particle shape must be chosen to couple preferentially into the substrate rather than backscatter into free space [69].

We now consider the case of an isolated nanoparticle on top of a thin film of absorbing material, as illustrated in Fig. 2.3 (adapted from [32]). Incident light is scattered off the object into a distribution of optical modes within the semiconductor. When the semiconductor is surrounded by index contrasting layers this structure can act as a waveguide. A fraction of the total power $\rho$ scattered off the nanoparticle will couple to the escape cone $\left(\rho_{0}\right)$ and a fraction $\rho_{j}$ to each guided mode, so that $\sum_{j=0}^{N} \rho_{j}=1$ where $N$ is the number of modes in the semiconductor. We call the effective size of the scatterer that couples to each mode the incoupling cross section $\sigma_{i n c, j}$, where

$$
\sigma_{\text {inc }, j}=\sigma_{\text {scat }} f_{\text {substrate }} \rho_{j}
$$

When normalized by the geometrical size of the object, this gives the normalized incoupling cross section $Q_{i n c, j}=\frac{\sigma_{i n c, j}}{\sigma_{p h y s}}$. Each of these modes will have a characteristic overlap with the semiconductor that describes the fraction of energy absorbed in the semiconductor, $\Gamma_{s, j}$. For a given mode at one wavelength, the fraction of power absorbed in the semiconductor layer of the cell is given by 


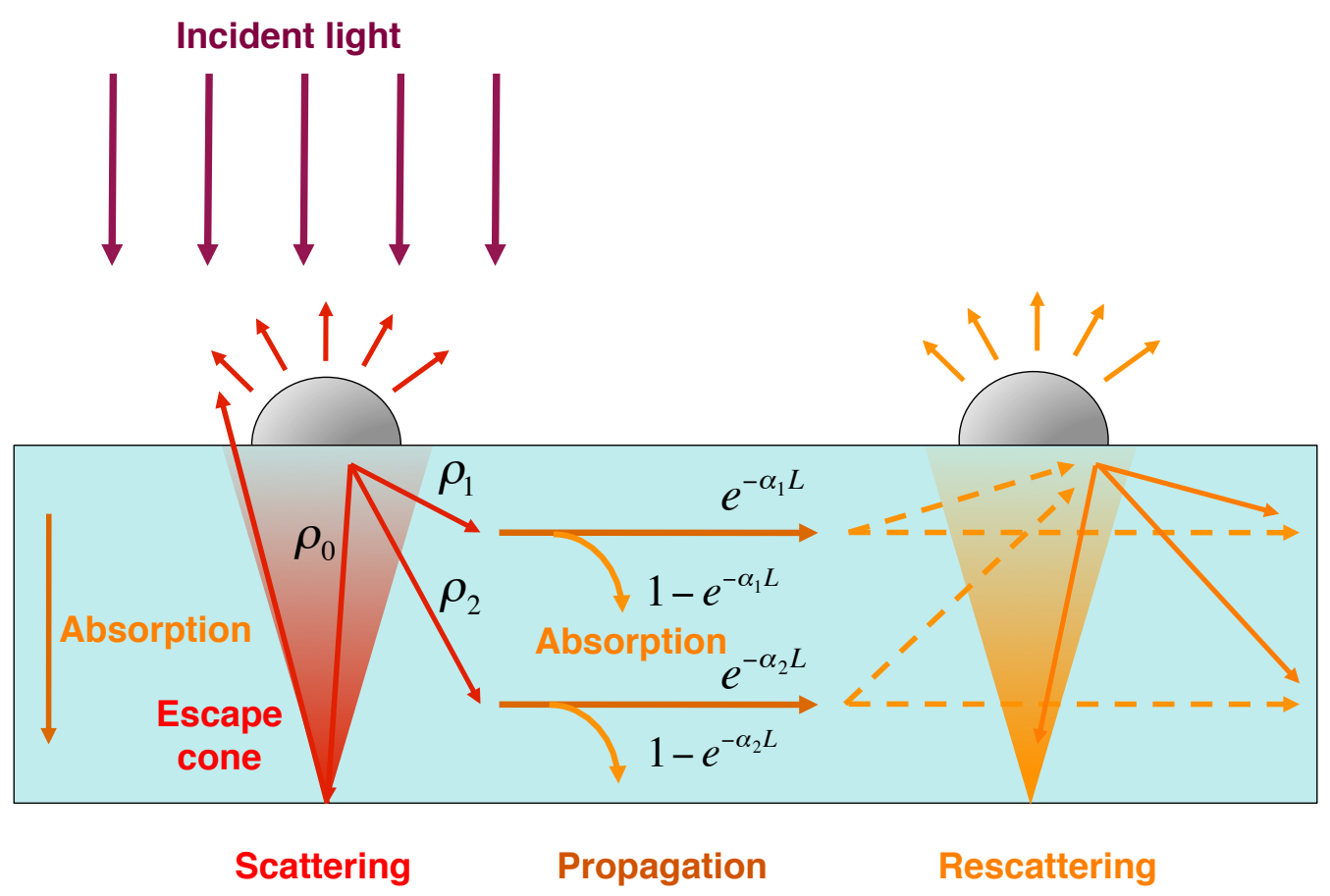

Figure 2.3. Diffusion model for light propagation inside the solar cell. Incident light is scattered off a scattering object, with a fraction $\rho_{0}$ coupling to the escape cone and $\rho_{j}$ to each guided mode. The modes propagate a distance $L$, with appropriate losses in the absorbing layer and cladding materials, until the next scattering object. At the next scattering object the mode is rescattered. Adapted from $[32]$. 


$$
A_{j}(\lambda)=\xi Q_{i n c, j} \Gamma_{s, j}
$$

The absorption coefficient in the semiconductor at one wavelength is then

$$
A(\lambda)=\sum_{j=0}^{N} A_{j}(\lambda)+\left(1-\xi Q_{\text {scat }}(\lambda)\right)(1-R(\lambda)-T(\lambda))
$$

Until now we have described only the effects of isolated scattering objects. In the limit where the scatterers act completely independently (and there is no effect from collective modes of the scatterers), the scattering objects should be placed $\xi Q_{\text {scat }}$ apart, effectively covering the entire surface. This case is similar to the first case of particles on a thick cell. The second case, where the absorbing layer is a thin waveguide, is illustrated in Figure 2.3 [32]. After the initial scattering event the propagating modes can rescatter from the object. A particular mode may continue to propagate past a neighboring scattering object, or it may be rescattered into either the escape cone or the other allowed modes of the structure. The design challenge lies in finding arrays of scatterers that efficiently incouple sunlight to the modes of the cell without increasing the outcoupling of the propagating modes. The opposite limit, where the scatterers are close together and exhibit near field coupling, is outside the scope considered here.

The exact structure of the scattering and absorbing layers can also modify both the types of modes excited and their semiconductor overlap $\Gamma_{s}$. The back cladding of the cell could be a metal or a transparent conducting oxide (TCO), for example, or a combination of both where the TCO acts as a spacer between the semiconductor and the metal, similar to the back contacts of many thin film Si cells. Ideally this back contact has low optical loss, allowing for waveguiding of modes rather than parasitic absorption. These spacer layers can also be used to tune the spectral scattering of the nanostructure: changing the refractive index of the surrounding material will shift the resonant frequencies and change the modal shape. This is discussed in more detail in Chapter 7 .

The choice of metal can also influence nanostructure design. Although Ag and 
$\mathrm{Au}$ are the most common plasmonic metals, elements such as $\mathrm{Al}$ and $\mathrm{Cu}$ have been shown to support surface plasmon resonances in the ultraviolet and visible range of the spectrum. $\mathrm{Al}$ and $\mathrm{Cu}$ are interesting plasmonic materials for photovoltaics since they are less expensive than $\mathrm{Ag}$ and $\mathrm{Au}$, but there are tradeoffs in the choice of metal for the cell. Ag generally has the lowest absorption losses of the four, but oxidizes quickly and must be well-passivated. Al forms a native oxide quickly which can weaken the effects of surface plasmon resonances on any surrounding semiconductor, so calculations must account for the presence of an oxide layer between the metal and the rest of the cell. Both $\mathrm{Au}$ and $\mathrm{Cu}$ have $\mathrm{SP}$ resonances in the visible, but $\mathrm{Cu}$ has higher losses than Au. Chapters 4 and 7 discuss this in more detail in specific geometries.

\subsection{Waveguide Modes}

The modes excited by the scattering object may be of two general types: waveguide modes that we call "photonic" due to their resemblance to the slab modes of planar dielectric waveguides, or SPP modes supported on the interface of the planar metal film and the semiconductor. In the absence of absorption in the semiconductor the SPP modes can propagate over distances of 10-100 $\mu \mathrm{m}$ before the Ohmic losses in the metal dominate. The SPP modes are tightly confined to the interface, with skin depths in the 10s of nanometers range. Enhanced absorption in the semiconductor may be due to either the photonic or the SPP modes.

By solving Maxwell's equations subject to the appropriate boundary conditions, we obtain the field intensity profiles for the various waveguide modes of a planar multilayer structure. Two independent sets of solutions exist, transverse magnetic (TM) and transverse electric (TE) with their magnetic field and electric field completely in the plane, respectively. Fig. 2.4 shows the modal profiles (calculated from simulation) at $\lambda=920 \mathrm{~nm}$ with both TE and TM polarizations for a $200 \mathrm{~nm}$ thick layer of c-Si with a $60 \mathrm{~nm}$ antireflective (AR) coating on the top and either air ((a) and (c)) or 300 $\mathrm{nm}$ of $\mathrm{Ag}((\mathrm{b})$ and $(\mathrm{d}))$ on the back side. These modal profiles represent the power 

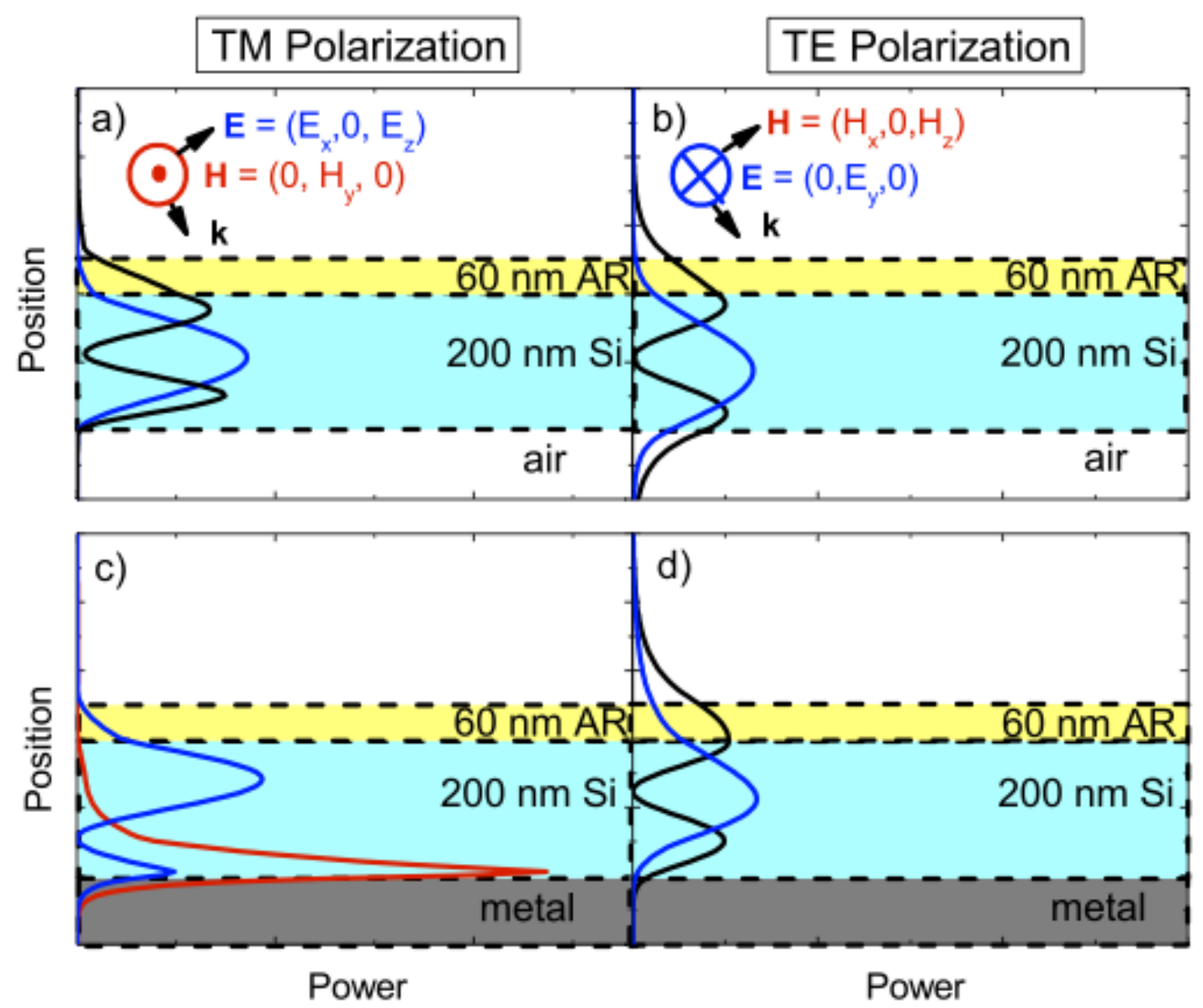

Figure 2.4. Modal profiles at $\lambda=920 \mathrm{~nm}$ for TM and TE polarizations, with and without metal back contacts. Profiles are normalized to equivalent power under the curve, and are calculated from postprocessed finite difference time domain simulation. 
in the mode as a function of position in the waveguide, and are normalized to the same integrated power to compare their shapes. In all cases we see that the modes are largely within the Si-AR core and decay more sharply into the surrounding air or Ag. The number of modes present in the waveguide depends on both the thickness of the slab and on the wavelength of the incident light.

In Chapter 5, we will compare the shapes of the modes to predict which modes will be most advantageous for optical absorption enhancement in the semiconductor. For now, we note simply that the photonic modes in Figs. 2.4 (a), (b), and (d) have similar shapes to each other, with varying decay outside the semiconductor. With the addition of a metal interface (Fig. 2.4(d)), the TE mode changes its shape slightly, but there is no substantial change in the number of accessible modes. In the TM case (Fig. 2.4(c)), a fundamentally different type of mode is visible when one of the interfaces is metal: the surface plasmon polariton mode which is tightly confined to the semiconductor-metal interface and decays exponentially from each side of the boundary. The lowest-order photonic mode under TM polarization changes shape as well, introducing a node within the Si just above the interface.

To further understand the SPP mode, we consider the field components in a simple two-material structure with semi-infinite metal and semiconductor layers. For TM modes, only $E_{x}, E_{z}$, and $H_{y}$ are non-zero. Enforcing continuity of the tangential component of $\mathbf{E}$ (i.e. $E_{x, m}=E_{x, s}$ ) and the normal component of $\mathbf{D}$ (i.e. $\epsilon_{m} E_{z, m}=$ $\left.\epsilon_{s} E_{z, s}\right)$ at the interface yields

$$
\frac{k_{z, m}}{k_{z, s}}=\frac{\epsilon_{m}}{\epsilon_{s}}
$$

which can be satisfied for a metal interface because $\Re\left[\epsilon_{m}\right]<0$ and both $\Re\left[k_{z, m}\right]>0$ and $\Re\left[k_{z, s}\right]>0$. The $H_{y}$ component in both materials must satisfy the wave equation.

$$
k_{z, i}^{2}=\epsilon_{i}\left(\frac{\omega}{c}\right)^{2}-k_{x}^{2}
$$

Combining these relations yields the surface plasmon dispersion relation 


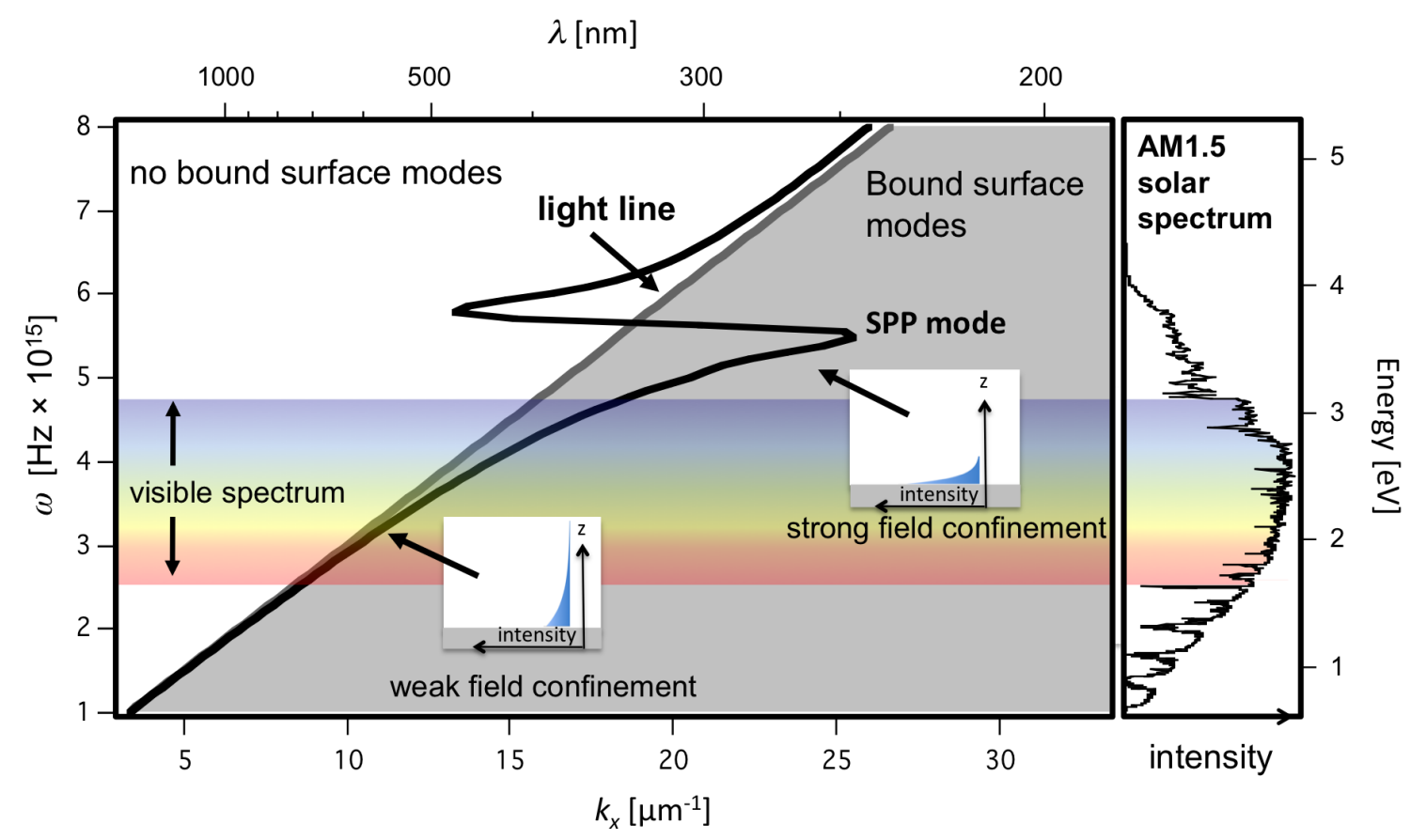

Figure 2.5. Surface plasmon polariton dispersion relation for an $\mathrm{Ag} /$ Air interface. Black (grey) line corresponds to the dispersion relation for the SPP mode (light line). A significant fraction of the solar spectrum (right part of figure) has access to the bound SPP mode.

$$
k_{x}=\frac{\omega}{c} \sqrt{\frac{\epsilon_{m} \epsilon_{s}}{\epsilon_{m}+\epsilon_{s}}}
$$

Fig. 2.5 shows the dispersion relation for an SPP mode at the Ag/Air interface, with the solar spectrum and visible spectrum highlighted. Modes to the right of the light line, the line describing light propagation in air, are bound to the interface. At low frequency, the SPP modes are close to, but to the right of, the light line, $k_{x}=\frac{\omega}{c} \sqrt{\epsilon_{s}}=\frac{\omega}{c}$. At resonance, where $\epsilon_{m}=-\epsilon_{s}$, the modes are highly confined with large wave vectors, and their propagation lengths are very short. The position of resonance depends on the optical properties of the metal and the semiconductor, so appropriate material and thickness choices will allow the amount of light confinement to be tailored for the most efficient use.

For the TE case, no bound surface modes exist. Recall that only $E_{x}, E_{z}$, and 
$H_{y}$ components are present, and that $E_{x}=E_{z}=H_{y}=0$ for this case. Enforcing continuity at the interface for the $E_{y}$ and $H_{x}$ terms yields

$$
E_{0}=k_{z, m}+k_{z, s}=0
$$

Because both $\Re\left[k_{z, m}\right]>0$ and $\Re\left[k_{z, s}\right]>0$, we must have $E_{0}=0$, and thus no surface mode exists under TE polarization.

\subsection{Conclusions}

This chapter shows a framework for the design of plasmonic solar cells, where the unique scattering properties of metal nanostructures and waveguide modes can be used to enhance absorption in thin semiconductor regions of solar cells. In particular, a wide variety of variables for nanostructure design can be explored, including individual nanostructure shape, coupling to waveguide modes, arrangement of nanostructures into patterns, and the inclusion of spacer layers. All of these will be discussed in later chapters, and analyzed both via numerical calculations and via experimental measurements. 


\section{Chapter 3}

\section{Numerical Methods}

\subsection{Introduction}

While analytic methods for solving Maxwell's equations are useful for studying and understanding optics in simple systems, more complex systems often necessitate numerical solutions. Finite element methods are flexible, and capable of modeling complex structures with precise control of the materials properties at each location. The techniques described here employ the finite-difference time domain (FDTD) method, which discretizes the system and explicitly solves the time-differential form of Maxwell's equations, producing solutions of the $E$ and $H$ fields everywhere in the computational domain. Another advantage is that since each material is explicitly defined over the grid cells, a wide variety of materials can be modeled naturally (although with caution to properly account for dispersion in the time domain). Additionally, broadband source conditions can be used, allowing for a full range of frequency response to be extracted from a single simulation, provided that the materials can be modeled accurately over the wavelengths of interest [72].

However, as the $E$ and $H$ are produced at every gridpoint, which must be sufficiently fine to resolve both the wavelength and the shape of the structure, the unprocessed output from FDTD can be intractably large. Post-processing techniques which compress the output are thus necessary to build up quantitative understanding with FDTD. With ongoing improvements in both nanoscale fabrication and computational power, it is natural to connect the output of FDTD simulations to practical and useful figures of merit. This chapter describes the development of two different post-processing techniques: Fourier mode spectral analysis (FMSA) and generation 
rate calculation. The FMSA method allows us to quantitatively connect the individual modes of a waveguide to design figures of merit such as the modal propagation length or incoupling of different scatterers. Calculation of the aggregate generation rate for a given device structure allows us to directly connect nanostructure designs to the maximum achievable short circuit current density of a solar cell. Specific examples will be given in this chapter to clarify each method, with detailed application to follow in later chapters.

\subsection{Fourier Mode Spectral Analysis}

We developed an approach that allows for the modal decomposition of scattered light into the SPP and photonic modal components, using a post-processing method of Fourier Mode Spectral Analysis (FMSA) to calculate the spectral frequency of the guided modes. This technique allows us both to analyze features of each mode, such as the spatial energy density profile and the propagation lengths in the waveguide, and to connect to other figures of merit such as the incoupling efficiency for a variety of scattering objects. Although there are analytic treatments for analyzing mode coupling via an overlap integral between the scattered fields of a nanostructure and the modes of a waveguide, complex systems benefit from the numerical treatment in FDTD $[73,74]$.

For solar applications, our aim is to use the FDTD method quantitatively to assess the incoupling cross section $\sigma_{i n c}$ of various nanostructures across the solar spectrum. Incident sunlight will scatter off a nanostructure into the various waveguide modes of the cell, which will then be absorbed as they propagate in the plane. As a first step to analyzing this problem, we focus on the analysis of incoupling, and how strongly a particular nanostructure couples to a particular mode [75, 76, 77]. Nanostructures could then be designed to selectively couple incident light into the desired mode, i.e. those with longer propagation lengths or better overlap with the semiconductor. The incoupling cross section described here corresponds to the areal flux of incident light that is "captured" by the object, which may be much larger than its physical size. 


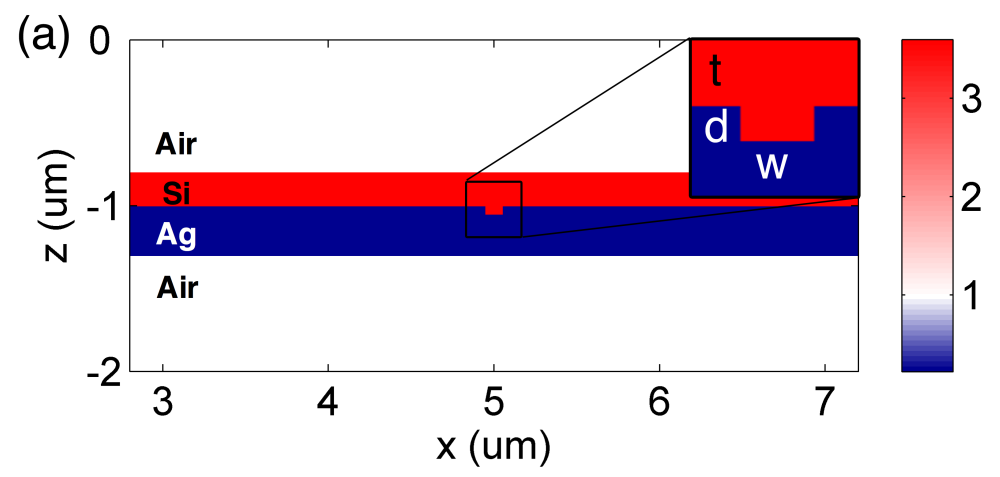

Figure 3.1. Schematic of sample structure, consisting of $200 \mathrm{~nm}$ of Si on $\mathrm{Ag}$ with a $100 \mathrm{~nm}$ wide and $50 \mathrm{~nm}$ deep groove in the Ag. The color scale is the refractive index.

This type of analysis is potentially applicable to many types of waveguide devices, whether solar cells, photodetectors, or color filters. The goal of this section is to describe our method for identifying the modes, assessing the incoupling ability of nanostructures, and for tracking the dissipation of power that enters the device.

FDTD is a time domain technique, which necessitates modeling of the dispersion for each material in the computation domain. For computational ease and accuracy in the time domain, the materials are modeled with analytical, physical fits to an empirical data set rather than explicit empirical data. If the simulations are monochromatic, and the dispersion of the material is weak, then it may be sufficient to approximate its model with a constant refractive index at each wavelength simulated. While simulation packages may automatically fit input data to an analytic model, this should be used with caution. First, the error in complex index from a poorly fit model may be more substantial. Second, if a broadband impulse source is used, care must be taken to ensure accurate modeling of the materials. In both cases multiple monochromatic simulations may help, as the error from assuming a constant refractive index may be less than the error from a poorly fit model. For the monochromatic simulations described here, $\mathrm{Ag}$ and $\mathrm{Al}$ are modeled as Lorentz-Drude dielectric functions using the method of Rakic et al. [78], and the optical constants for c-Si and GaAs from 400 to $1100 \mathrm{~nm}$ are taken from Palik [79] and approximated as constant refractive index materials where the index changes with each individual wavelength simulation. 
Figure 3.1 shows the schematic of a typical structure we simulated, a $200 \mathrm{~nm}$ thick layer of Si on $300 \mathrm{~nm}$ of $\mathrm{Ag}$ with a single $100 \mathrm{~nm}$ wide by $50 \mathrm{~nm}$ deep groove cut into the Ag. Each simulation generates a map of time-harmonic electromagnetic fields for a steady-state plasmonic device with continuous wave, monochromatic input. The structure is illuminated at normal incidence by a continuous wave source $(\lambda=1000$ $\mathrm{nm}$ ) polarized transverse magnetic (TM), i.e. with the $H$ field directed along the long axis of the groove. The source plane is located $2 \mu \mathrm{m}$ above the $\mathrm{Si} / \mathrm{Ag}$ interface. In Figure 3.1, the interface is located at $\mathrm{x}=-1 \mu \mathrm{m}$, and the beam is launched at $\mathrm{x}$ $=1 \mu \mathrm{m}$. The two-dimensional simulation volume is $10 \mu \mathrm{m}$ long, with the scattering object at the center, and $4 \mu \mathrm{m}$ tall. A uniform mesh size of $5 \mathrm{~nm}$ is used for the entire volume, and perfectly matched layers (PML) are used at the boundaries.

Incident light is scattered from the groove and may be coupled into propagating modes, which are subsequently absorbed in either the semiconductor or the metal. Some of the optical power backscattered at angles near normal is lost by transmission through the top surface. Additionally, there are fundamental thin film processes such as reflection, transmission, and absorption, that occur in the device without necessarily interacting with the scatterer.

\subsubsection{Isolation of Scattered Fields}

A full-field simulation of this device generates a complicated field pattern from the interference of the incident beam $\left(\mathbf{H}_{i n c}\right)$ with the fields reflected from the smooth metal surface $\left(\mathbf{H}_{r e f}\right)$ and those scattered by the groove $\left(\mathbf{H}_{\text {scat }}\right)$. Since we are working with complex-valued time harmonic fields, it is straightforward to isolate the scattered fields by employing a linear subtraction method. We first perform a full-field simulation where the interference pattern is generated by the sum of these three component fields $\mathbf{H}_{\text {total }}=\mathbf{H}_{\text {inc }}+\mathbf{H}_{r e f}+\mathbf{H}_{\text {scat }}$. A second control simulation is then performed with a smooth metal surface without any groove. Here $\mathbf{H}_{\text {control }}=\mathbf{H}_{\text {inc }}+\mathbf{H}_{\text {ref }}$. The other parameters are identical, so we determine the scattered fields by subtraction, $\mathbf{H}_{\text {scat }}=\mathbf{H}_{\text {total }}-\mathbf{H}_{\text {control }}$. This solution is valid for regions outside the groove.

This process is illustrated in Fig. 3.2, showing that the scattered fields in a semi- 

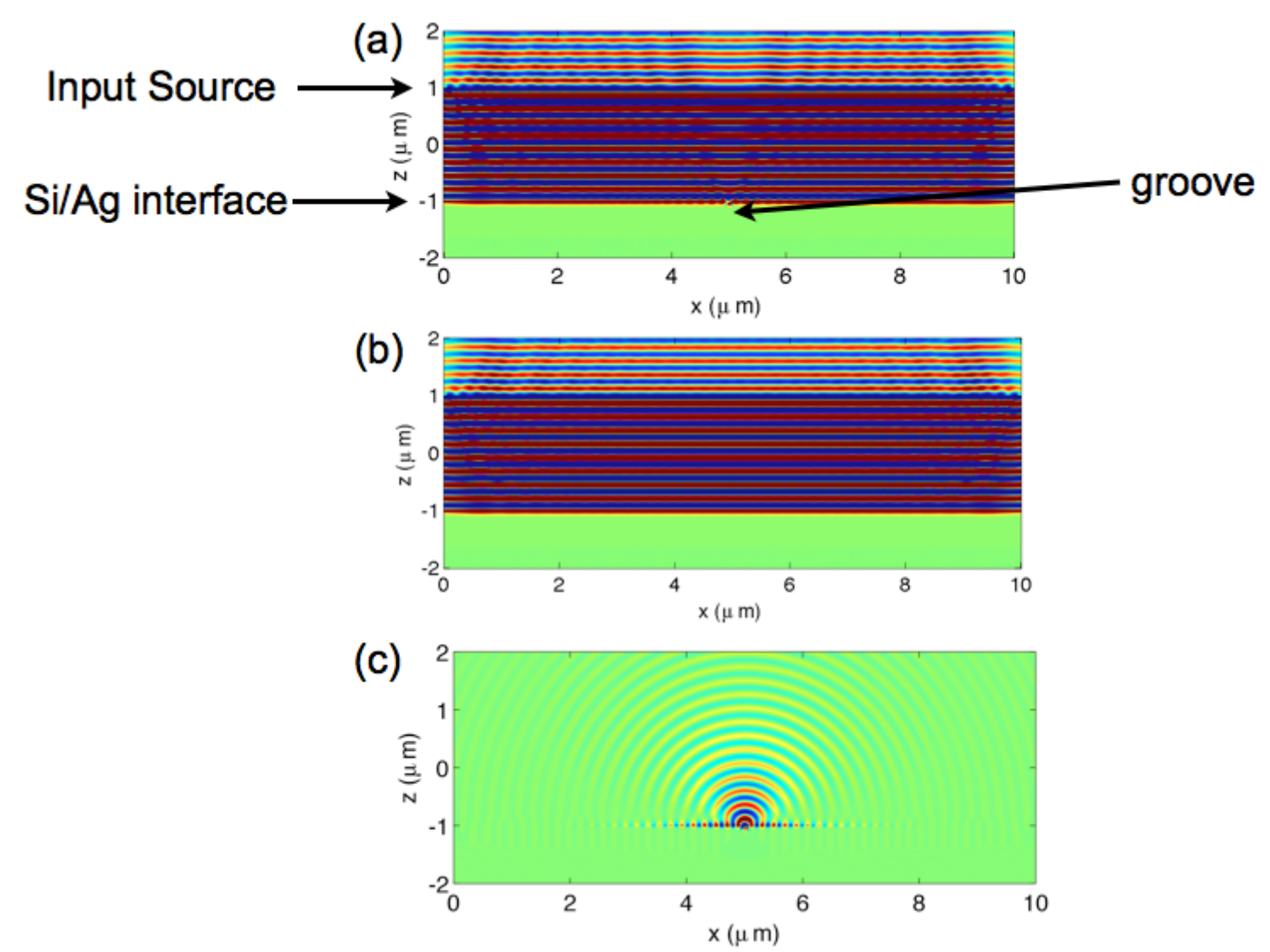

Figure 3.2. Isolation of scattered fields via subtraction. The simulation is a semi-infinite slab of $\mathrm{Si}$ on an $\mathrm{Ag}$ film, with a single groove cut into the $\mathrm{Ag}$ at the center. All three images show the $H_{y}$ fields obtained from simulation. (a) Semi-infinite structure with groove. (b) Reference structure without groove. (c) Scattered fields obtained via subtraction. An SPP mode is excited along the Si/Ag interface, as well as backscattering from the groove. 
infinite film can be isolated. In the original simulation, Fig. 3.2(a) the groove does scatter incident light, but the standard processes such as reflection and absorption interfere with the scattered fields, making the modes difficult to visualize. The close similarity to the control simulation (Fig. 3.2(b)) shows the extent that reflection and unperturbed absorption dominate the map of the $H$ field. Figure 3.2(c) shows the isolated scattered fields for the semi-infinite case, where only an SPP mode is excited along the interface. These images also illustrate one of the common issues in FDTD simulation of isolated nanostructures: plane wave input sources cannot be used with PML simulation boundaries, as this can lead to unwanted reflection from the edges. In this case the subtraction method eliminated the error, as the same issue was present in the reference simulation in Fig. 3.2(b). To avoid this problem, the rest of this chapter uses Gaussian input sources with a sufficiently wide half width, to appear planar over the size of the scattering object. The simulation volume is chosen to be appropriately large to both minimize reflections from the input source at the boundaries and to minimize the deviations from a plane wave over the region of interest.

\subsubsection{Fourier-Space Mode Filtering}

In the thin film case, other modes besides the SPP mode will be excited in the c-Si structure. Figure 3.3 shows the isolated scattered fields for two different nanostructures in the Ag film, a groove and a ridge. The remaining interference pattern is due to the presence of multiple modes in the waveguide. The difference in these two scattering responses motivates the development of our FMSA technique to decompose the individual modes. The post-processing method takes a map of each of the three electromagnetic field components present under TM polarization $\left(E_{x}(x, z), H_{y}(x, z), E_{z}(x, z)\right)$ as input, which are then transformed to wavevector space $\left(e_{x}(x, k), h_{y}(x, k), e_{z}(x, k)\right)$ using a one-dimensional fast Fourier transform (FFT). The $\mathrm{x}$-position remains unchanged. To visualize the energy content of the modes, we calculate a power spectrum for each field: $\left|h_{y}(x, k)\right|^{2}=h_{y}(x, k) \cdot h_{y}^{*}(x, k)$.

Figure 3.4(a) shows these Fourier-space power spectrum map, calculated at $\lambda=$ 


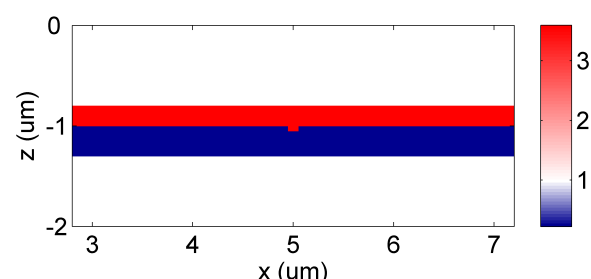

(a) Refractive index profile with groove.

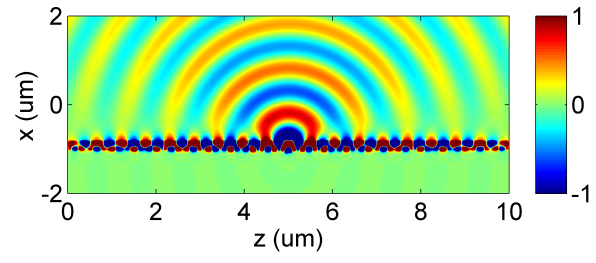

(c) Scattered fields from groove.

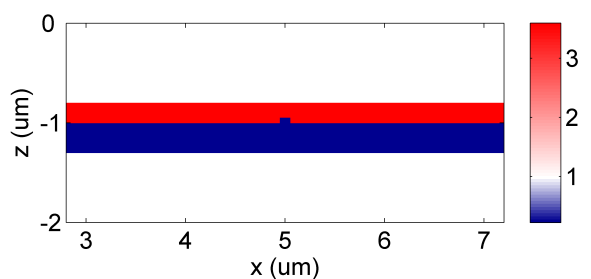

(b) Refractive index profile with ridge.

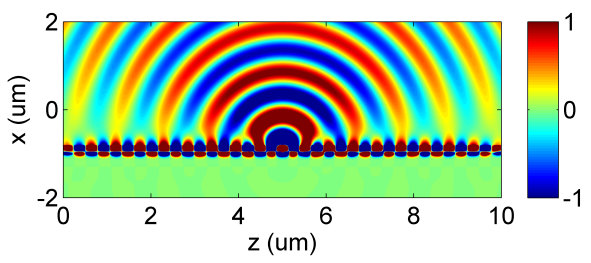

(d) Scattered fields from ridge.

Figure 3.3. Calculated scattered $H_{y}$ fields in $200 \mathrm{~nm}$ of c-Si from a $100 \mathrm{~nm}$ wide by $50 \mathrm{~nm}$ deep groove and a $100 \mathrm{~nm}$ wide by $50 \mathrm{~nm}$ tall ridge in a $200 \mathrm{~nm}$ thick c-Si film on Ag.

$1000 \mathrm{~nm}$. The horizontal axis is the spatial frequency, and the vertical axis records the position in the waveguide. We see two bright "spots" along the frequency axis, centered at $2 \mu \mathrm{m}^{-1}$ and $4.2 \mu^{-1}$; each of these peaks represents a mode. Along the $\mathrm{x}$ position axis, the mode at $2 \mu \mathrm{m}^{-1}$ has one bright spot vertically, representing the energy profile within the core of the waveguide, and a second one at the metalsemiconductor interface. This spatial mode profile is somewhat similar to that of a conventional dielectric waveguide mode, and by analogy we designate it as a photonic mode. The peak at $4.2 \mu^{-1}$ has a higher spatial frequency and is localized at the interface, indicating that it is the SPP mode.

Cross sections of this data set along either axis reveal other characteristics of the system. Figure 3.4(b) plots the Fourier power spectrum $\left|h_{y}\right|^{2}$ as a function of spatial frequency, at three different x-positions in the device. At the top Si/air interface $(\mathrm{x}=-0.8 \mu \mathrm{m})$ we observe only the photonic mode, in the middle of the Si layer we see the SPP mode begin to come in, and at the $\mathrm{Si} / \mathrm{Ag}$ interface $(\mathrm{x}=-1 \mu \mathrm{m})$, we observe a superposition of both modes. Figure 3.4(c) represents the other crosssection of Figure 3.4(a), the spatial mode profile, or Fourier power spectrum as a 

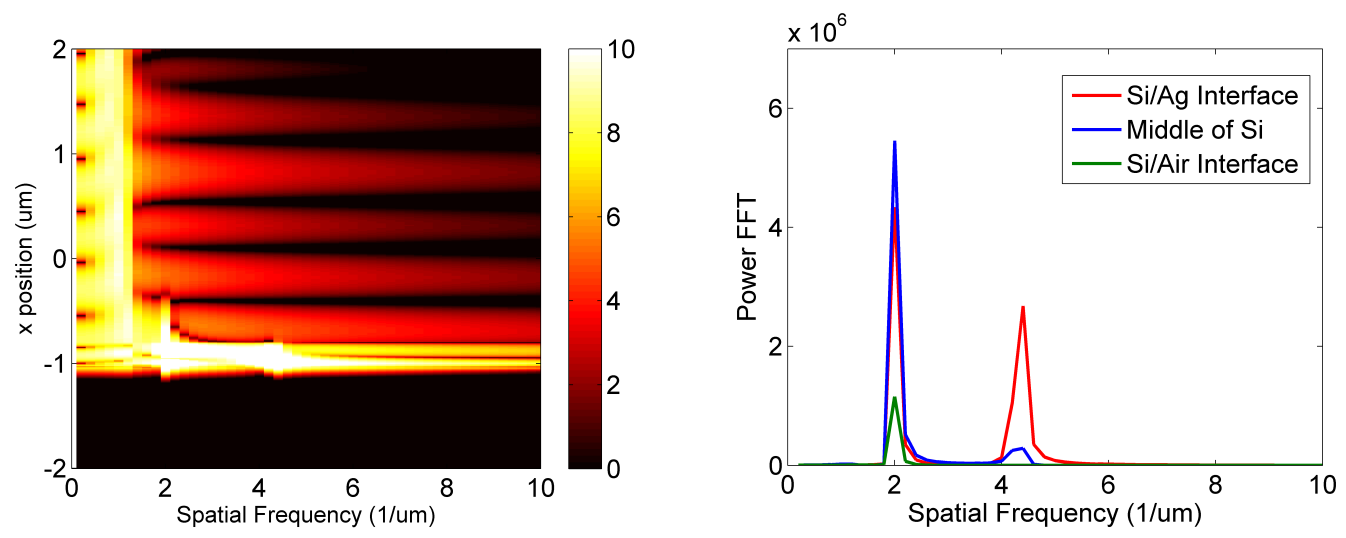

(a) One dimensional Fourier transform of Fig. (b) Frequencies of the modes present at differ$3.3(\mathrm{a})$, where the $\mathrm{z}$ axis is transformed and the ent positions in the waveguide, obtained from $\mathrm{x}$ axis is unchanged.

a horizontal cross-cut of (a).

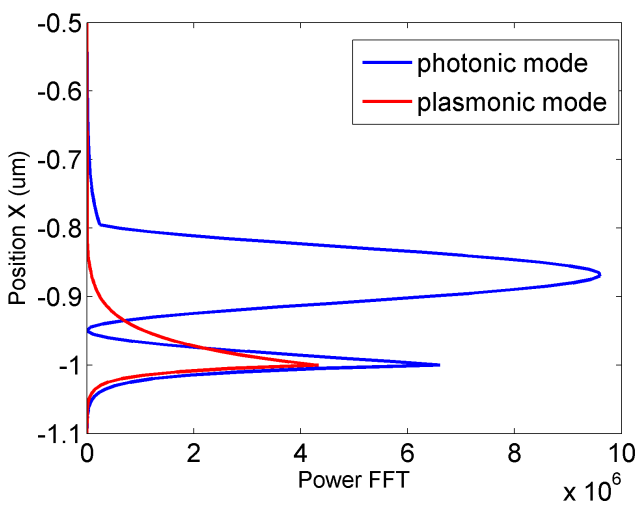

(c) Mode profiles obtained from a vertical cross cut of (a), taken at the frequencies of the modes identified in (b).

Figure 3.4. FMSA method for isolating waveguide modes, shown at $\lambda=1000 \mathrm{~nm}$ for $200 \mathrm{~nm}$ of Si on $300 \mathrm{~nm}$ of $\mathrm{Ag}$. 
43

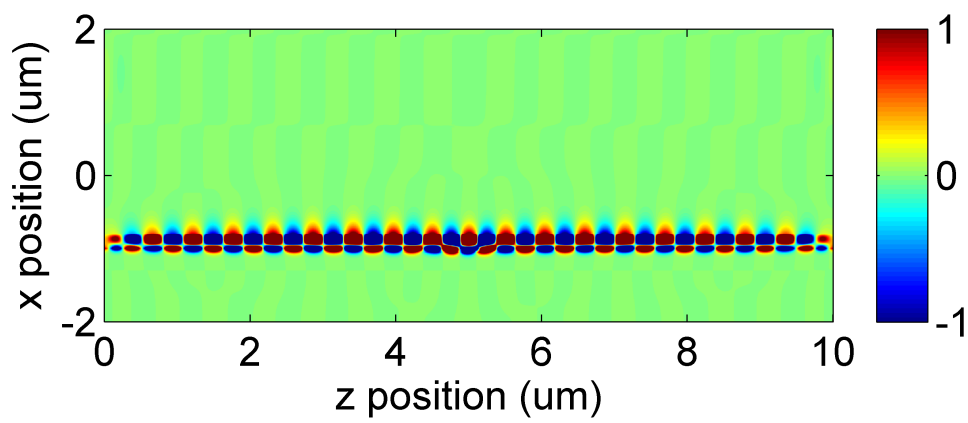

(a) $H_{y}$ field of photonic mode

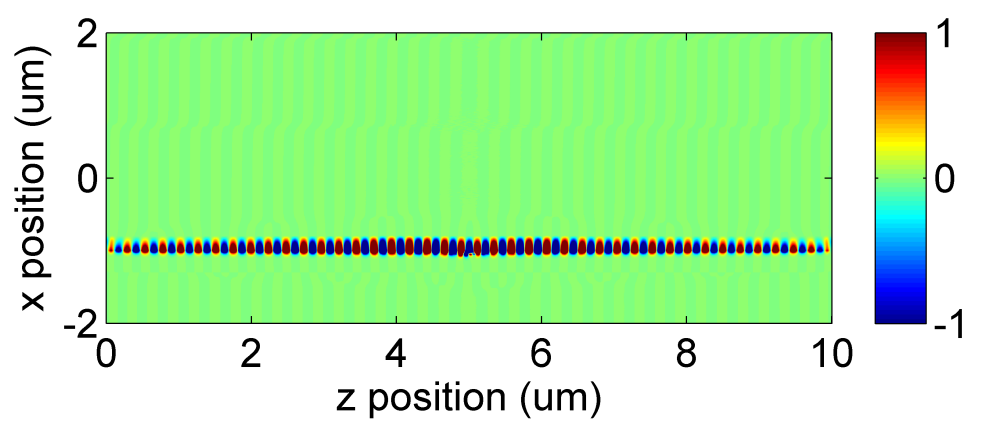

(b) $H_{y}$ field of SPP mode

Figure 3.5. $H_{y}$ field maps of isolated modes after filtering in Fourier space and transforming back to real space.

function of position. The cuts are taken at the peak frequencies observed in Figure 3.4(b), allowing us to visualize the power localization within the waveguide. The SPP peak at $4.2 \mu^{-1}$ is shown in red; it is confined to the $\mathrm{Si} / \mathrm{Ag}$ interface, and the power falls off exponentially on each side. The photonic mode at $2 \mu \mathrm{m}^{-1}$ is plotted in blue, and we confirm that it is the lowest-order mode.

Since the modes are distinguishable by their wavevector $k$ in Fourier space, we can perform modal analysis that is not possible in Cartesian $(x, z)$ space. Our method uses a Fourier-space notch filter to isolate each mode. The notch filter is perfectly 


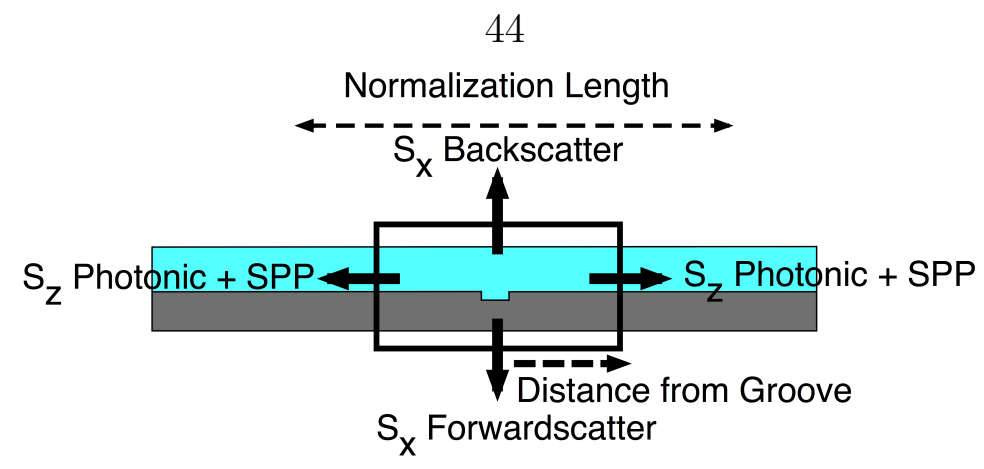

Figure 3.6. Schematic of calculation of power flow away from the scattering object.

rectangular, and of the form

$$
f(k)= \begin{cases}0 & \text { if }|k|<k_{\text {low }} \\ 1 & \text { if } k_{\text {low }}<|k|<k_{\text {high }} \\ 0 & \text { if }|k|<k_{\text {low }}\end{cases}
$$

These filtered sets are then transformed back to real space, producing an image of each individual mode. Figures 3.5(a) and 3.5(b) show the $H_{y}$ component of the filtered fields after transforming back to Cartesian coordinates; the top image is the photonic mode, and the bottom the SPP mode.

\subsubsection{Incoupling Cross Sections}

We are most interested in connecting these data sets to the energy content of each mode and the incoupling efficiency of the scattering object. The energy flow in the structure is calculated using the time-harmonic form of Poynting's theorem

$$
<\mathbf{S}>=\frac{1}{2} \Re\left(\mathbf{E}_{\text {scat }} \times \mathbf{H}_{\text {scat }}^{*}\right)
$$

where $\mathbf{E}_{\text {scat }}$ is the scattered electric field ( $E_{x}$ and $E_{z}$ components) and $\mathbf{H}_{\text {scat }}^{*}$ is the complex conjugate of the magnetic field ( $H_{y}$ component). This calculation is done for each of the filtered modes.

We then draw an enclosing pillbox around the structure with a variable length $l \hat{z}$ 
and a height $h \hat{x}$ that is large relative to the exponential energy penetration depth in both $\mathrm{Si}$ and $\mathrm{Ag}$ and therefore encompasses the majority of the modal energy which extends outside the waveguide, and a variable length $l$. The line integral through the Poynting vector plot on each side of the box gives the time average power flow in that direction: the top measures the backscattered component, the bottom measures any transmission which occurs through the metal film, and the components flowing to the sides measure the power in the guided modes. Assume that the scattering object is centered at $(0,0)$, and the box is symmetric around the center point (although this is not a necessary assumption for the forward- and backscattering sides). A schematic of the box drawn around the film is shown in Figure 3.6.

$$
\begin{gathered}
U_{\text {backscatter }}=\int_{-\frac{l}{2}}^{\frac{l}{2}} S_{x}\left(x=\frac{h}{2}, z\right) d z \\
U_{\text {guided }}=\int_{-h / 2}^{h / 2} S_{z}\left(x, z=\frac{l}{2}\right) d x
\end{gathered}
$$

The extent of the mode outside the semiconductor varies by wavelength, and so the pillbox height $h$ may vary across the spectrum. None of the modes extend significantly outside the $\mathrm{Ag} /$ air interface. At a given frequency and constant $h$, we step through a number of box lengths, thus monitoring the power flow as a function of distance from the groove. The entire data set is normalized by the incident power that hit the top of the device in a $1 \mu \mathrm{m}$ wide region surrounding the center point of the scattering object.

$$
U_{\text {incident }}=\int_{-0.5}^{0.5} S_{x, \text { incident }}(x=\mathrm{Si} / \text { air interface, } z) d z
$$

where $S_{x, \text { incident }}$ is from a separate control simulation of the propagating incident light in the absence of a device to account for the shape of the Gaussian input source.

The guided modes are lossy, so we calculate the attenuation during propagation by observing the power flow at multiple lengths $l$. The attenuation is assumed to be of exponential form, and so we can observe the power at points any distance from the groove and fit to extract the value at $\frac{l}{2}=0$. Figure 3.7 shows an example of the power 


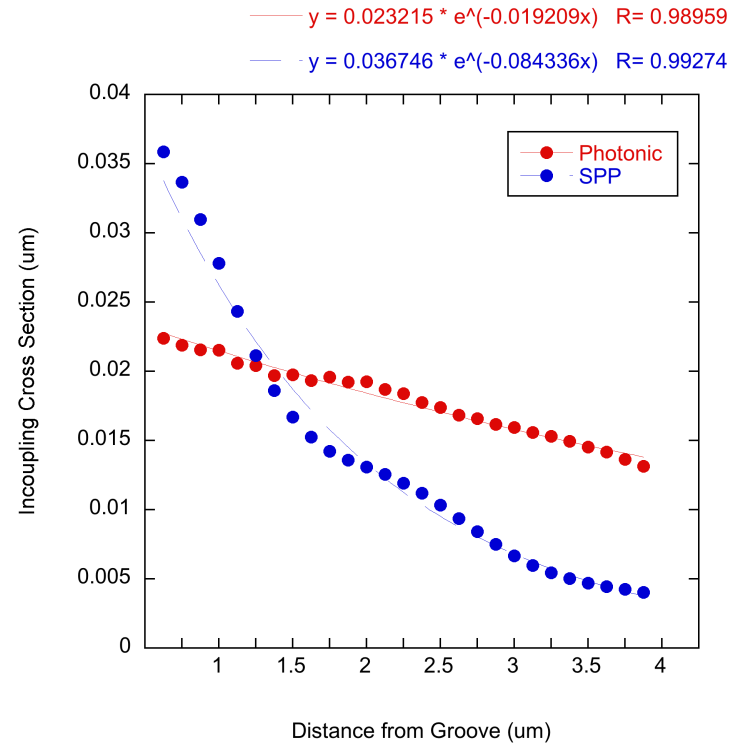

Figure 3.7. For each mode, the line integrals of $S_{z}$ through the width of the waveguide are plotted at increasing distance from the groove centerpoint. This measures the power decay in each mode, which we fit to an exponential curve to back out the value at the origin (groove mouth). All of the numbers are normalized by the power incident on the top of the Si over a $1 \mu \mathrm{m}$ area surrounding the groove to give an effective size. This data set is for $1000 \mathrm{~nm}$ incident light, and a $100 \mathrm{~nm} \times 50 \mathrm{~nm}$ groove in $200 \mathrm{~nm} \mathrm{Si}$, and shows only the modes moving in the $+\mathrm{z}$ direction.

decay as a function of groove distance for $\lambda_{\text {excitation }}=1000 \mathrm{~nm}$. The slices need to start some distance away from the groove for the modes to be fully formed, and with 5 $\mu \mathrm{m}$ propagation lengths in our simulation volume we calculate the normalized power flow slices for $0.625 \mu \mathrm{m}<\frac{l}{2}<4.5 \mu \mathrm{m}$. The same calculation is done on each side of the groove, to account for modes launched in both directions on excitation. After normalization, we call this extracted value the incoupling cross section $\sigma_{i n c}$. This cross section describes the physical area of light harvested by the groove, which may be much larger than its physical dimensions. For a two-dimensional simulation, the cross section has units of length rather than area as in a three-dimensional simulation, and so it is comparable to an effective width of the structure. 


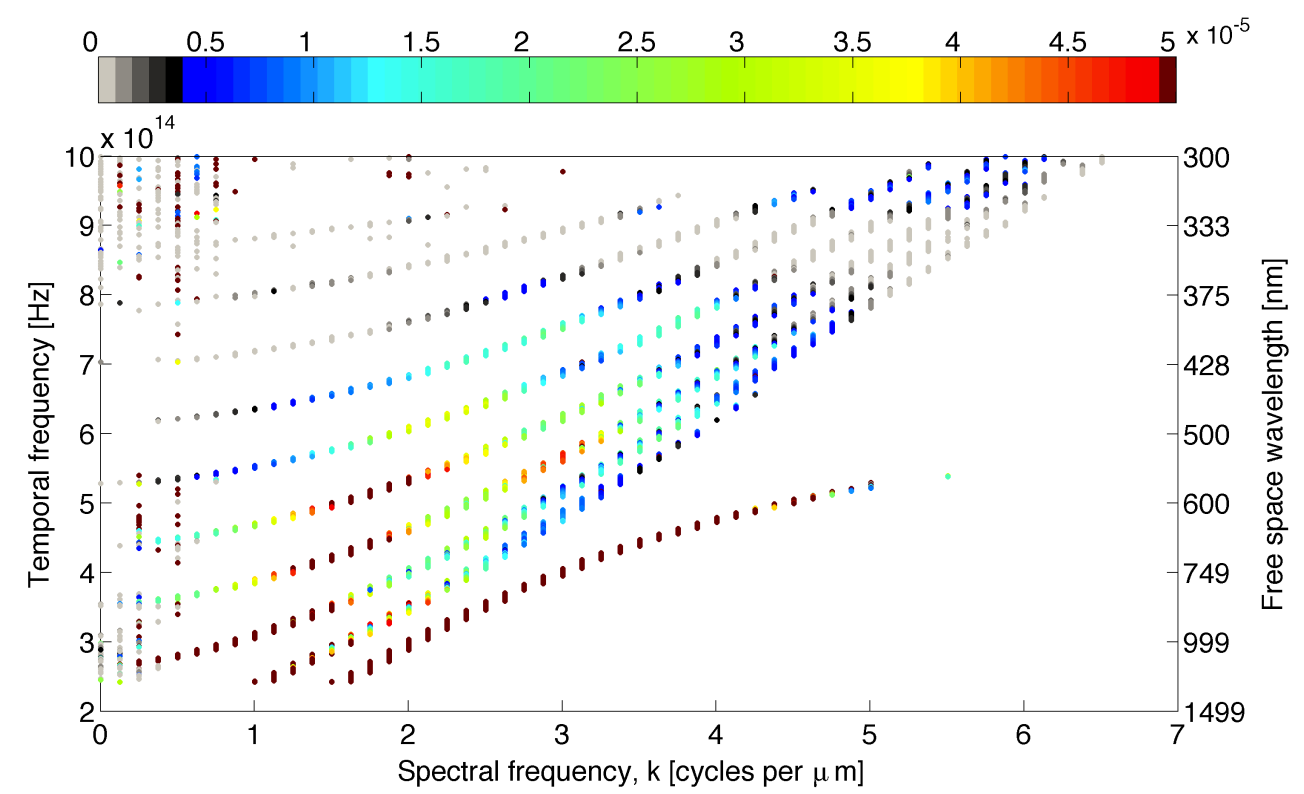

Figure 3.8. Dispersion relation constructed from a single simulation containing many temporal frequencies. The waveguide is a multimode metal-insulator-metal structure with an $800 \mathrm{~nm}$ Si Nitride core and Ag cladding. A $200 \mathrm{~nm}$ wide slit is cut through the top Ag, halfway through the $\mathrm{Si}_{3} \mathrm{~N}_{4}$ core.

\subsubsection{FMSA with Broadband Sources}

The FMSA method can also be applied to simulations done with broadband sources. At any temporal frequency contained in the pulse, the FMSA method can be applied to extract the spectral frequency of all the modes present in the waveguide, and used to build a quantitative dispersion relation as shown in Fig. 3.8. Each mode at each temporal frequency can be simultaneously analyzed for a figure of merit; the color scale at each point in Fig. 3.8 is the energy incoupled from the input source of that temporal frequency to the mode of that spectral frequency. When combined with FDTD methods, the FMSA method thus allows for the construction of dispersion relations for complex geometries that are difficult to solve analytically. 


\subsection{Absorption}

The second computational method is the direct calculation of generation rates for a given solar cell design. This is closely connected to an experimentally observable property: the photocurrent of an operating solar cell. FDTD is well-suited to this problem for several reasons. The flexibility in defining structures allows for a wide variety of light trapping surfaces to be evaluated without assumptions of regularity or symmetry. Since the materials are defined explicitly at every gridpoint, it is straightforward to separate absorption in parasitic materials (such as metal) from absorption in photocurrent-generating materials, even over irregularly shaped features. Furthermore, since the field profiles are calculated at all points in the computational domain, cross-sectional images of the absorption can be mapped to directly quantify both the magnitude and location of absorption within each device layer. Integrating over the absorption in the photocurrent-generating region for a monochromatic source gives the spectral response; weighting a series of monochromatic simulations by the solar spectrum can give the generation rate.

The absorption can be calculated from the divergence of the time-dependent Poynting vector $u_{a b s}=\nabla \cdot \mathbf{S}$. The method requires a map of $E$ and the complex refractive index over the computational domain. This can be determined directly from the simulation output, as the divergence of the Poynting vector at each grid point is equivalent to the expression below

$$
u_{a b s}=\frac{1}{2}\left(\omega \epsilon^{\prime \prime}|\mathbf{E}|^{2}+\omega \mu^{\prime \prime}|\mathbf{H}|^{2}\right)
$$

where $\omega$ is the input source frequency, $\epsilon^{\prime \prime}$ is the imaginary part of the permittivity, and $\mu^{\prime \prime}$ is the imaginary part of the permeability. In most materials, $\mu^{\prime \prime}=0$ and the second term drops out. The absorbed power can be converted to photon flux for each wavelength.

$$
\Phi(\lambda)=\frac{u_{a b s}}{\hbar \omega} \Phi(\lambda)=\frac{1}{2 \hbar} \epsilon^{\prime \prime}|E|^{2}
$$




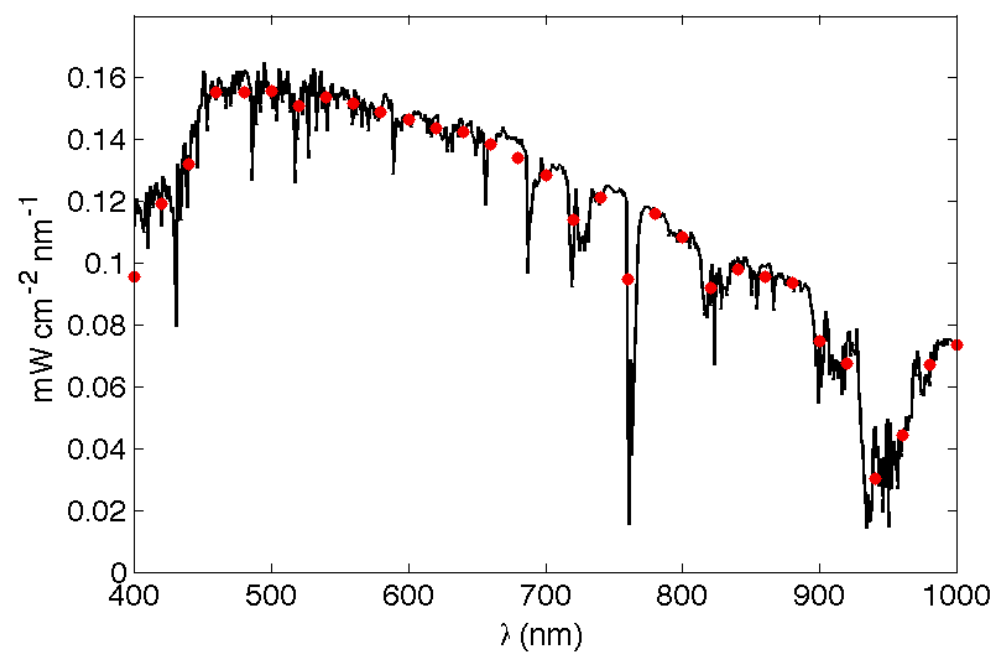

Figure 3.9. AM1.5 solar spectrum with wavelengths of simulations marked.

Plotting cross sections of the data set at this point allows for visualization of the total absorption. To isolate the absorption in a particular material, we use the refractive index monitor to build a matrix "mask" consisting of 1 s at each of the gridpoints matching that material and 0s elsewhere. Multiplying $\Phi$ by this mask isolates the photon flux in that material only. Under the assumption that every absorbed photon produces one electron in the semiconductor, this quantity is the same as the optical generation rate $G_{o p t}(\lambda)$. For either good quality materials or sufficiently thin layers, this can be a reasonable assumption that closely matches the real $J_{\text {sc }}$ of the solar cell, while for materials with poor collection this provides some insight into the relative importance of absorption and collection.

For the reasons discussed in Section 3.2 with materials modeling across broad spectral ranges, the majority of the simulations in this thesis were done with monochromatic input sources. Although FDTD uses coherent sources, and sunlight is incoherent, the method is still valid as the coherence length of sunlight is approximately 30 $\mu \mathrm{m}[80]$. Over the size scale of the structures considered here, this is not a significant source of error. Figure 3.9 shows the AM1.5 solar spectrum, with discrete simulation points marked across the 400 - $1000 \mathrm{~nm}$ spectral range. The solar spectrum is broad, 


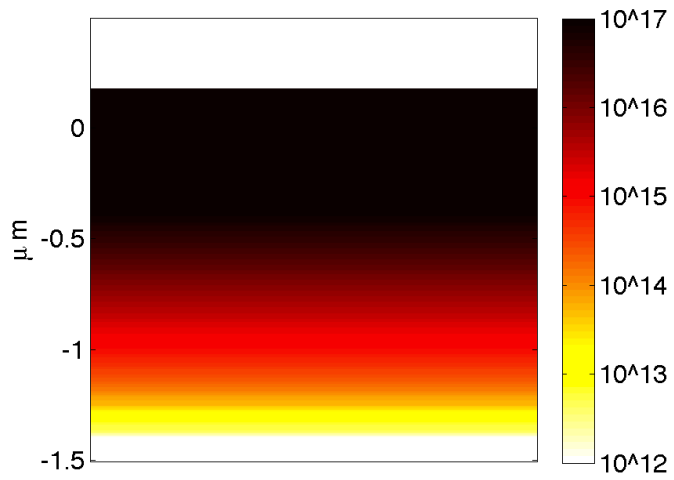

(a) Thick c-Si film.

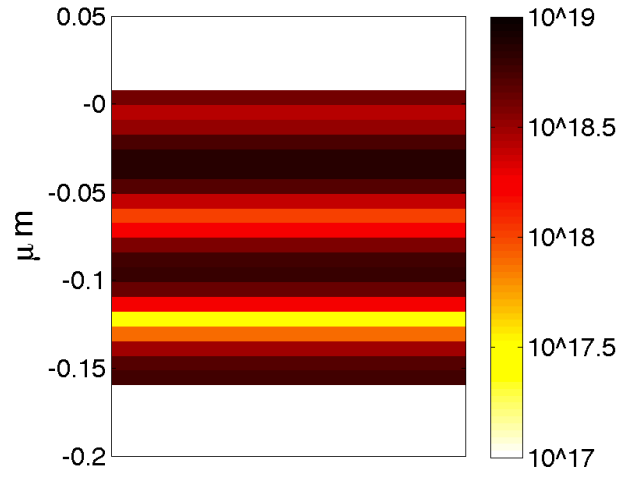

(b) Thin c-Si film with back reflector.

Figure 3.10. Generation rate maps for thick and thin c-Si.

and the power in the spectrum varies significantly with wavelength. To calculate an aggregate $G_{\text {opt }}$ we bin the power in the solar spectrum around each of the simulated points, and correct the input source power to the AM1.5 power in this spectral range. These solar spectrum weighted images can also be mapped, or integrated over volume to give the final figure of merit, $G_{\text {opt }}$.

For a standard thick film of material, light is absorbed exponentially from the front to back. For thin films, however, particularly those with back reflectors, the thin film will act like a Fabry-Perot cavity with multiple reflections within the film. For a given thickness, particular wavelengths will have particularly strong absorption. Similarly, there will be locations within the thickness of the cell with particularly strong absorption, and other bands of weaker absorption. Figure 3.10 shows a comparison of exponential absorption in a $1.5 \mu \mathrm{m}$ thick film of c-Si and thin film absorption in 150 nm thick film of c-Si with monochromatic input. Fig. 3.10(b) indeed shows bands of higher and lower absorption.

When more complex nanostructures are introduced, the absorption maps will be spatially inhomogeneous. Examples of solar spectrum weighted electron generation map profiles are shown in Figure 3.11. Only the generation in the semiconductor is shown, below $\mathrm{x}=-1$ is $\mathrm{Ag}$ (or air), and above $\mathrm{x}=-0.8$ is an anti-reflection (AR) coating and then air. The color scale is logarithmic, with generation rates mostly in the $10^{21}$ to $10^{22}$ electrons per $\mathrm{cm}^{3}$ per second range. The simulations are two- 


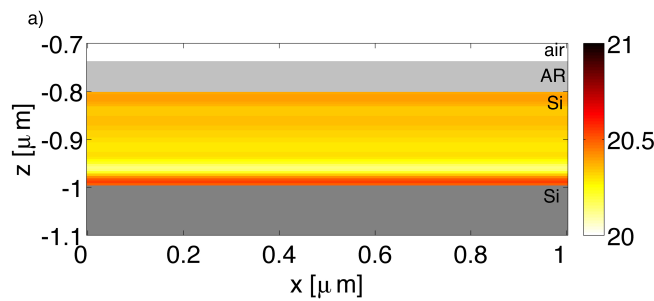

(a) Flat film.

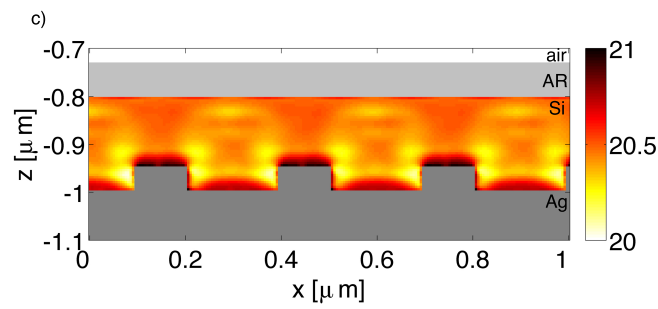

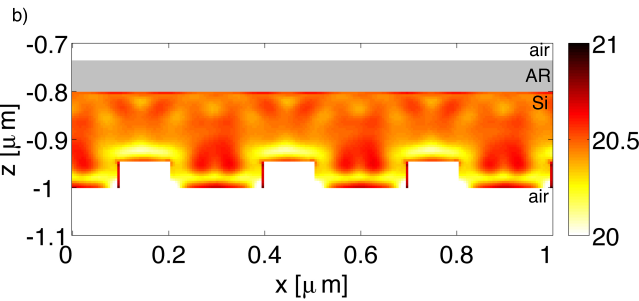

(b) Nanostructured pillars with air on the back, TM polarization.

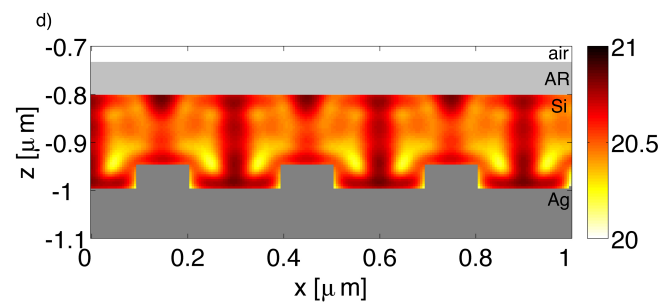

(c) Nanostructured pillars with $\mathrm{Ag}$ on the (d) Nanostructured pillars with $\mathrm{Ag}$ on the back, TM polarization. back, TE polarization.

Figure 3.11. Solar spectrum weighted generation rate. The color scale is logarithmic. The c-Si is $200 \mathrm{~nm}$ thick, and has an antireflection coating.

dimensional, so each pillar is semi-infinite in the out-of-page direction, which also explains why the cross sections of three-dimensional simulations in Fig. 3.10 have a lower order of magnitude. Figure 3.11(a) shows the electron distribution for a planar interface. The bands of varying absorption strength are due to the FabryPerot resonances within the film, which are strongly present at some wavelengths, but appear reduced in strength when summed over the whole range. Figure 3.11(b) shows the TM polarized case with air on the back interface (and hence no SPP excitation), while figures 3.11(c) and 3.11(d) show the generation rates for an $\mathrm{Ag}$ grating under TM and TE polarization.

The dense periodic arrays of nanostructures (Figures 3.11(b), 3.11(c), and 3.11(d)) all show the interference effects between the scattering objects quite strongly; the generation rates are periodic, with regions of high generation concentrated in the "cavity" between the ridges. Figure 3.11(b), in contrast to the others, has lower 
generation rates around the scattering objects and more concentrated generation in the bulk. Figure 3.11(c), the case with metal under TM polarization, shows very high generation rates near the metallic surface, consistent with SPP excitation and the high fields associated with subwavelength metal nanostructures. The TE case also has high generation in the vicinity of the particle, but its highest bands are in the cavity between centers.

\subsection{Conclusions}

This chapter described the connection between FDTD output and design figures of merit for two different applications. The first, based on Fourier space analysis of modes, will be applied in detail in the next chapter to study incoupling from single nanostructures into a thin film of c-Si on Ag. The second method, where the optical generation rate is calculated from the electric field will be applied to single nanostructures in the next chapter, then used in detail in later chapters to analyze periodic and random structures and compare to experimental photocurrent measurements. 


\section{Chapter 4}

\section{Incoupling from Single Isolated}

\section{Nanostructures}

\subsection{Introduction}

The waveguide modes discussed in Chapter 2 cannot be excited without a means to match the momentum of the incident light to the momentum of the mode. Historically, optical excitation of SPPs has been performed by prism coupling [35, 81] or by gratings, [82] which can be highly efficient but limited in both wavelength and angular range. More recently, phenomena such as extraordinary transmission through hole arrays and slits have been intensively studied [76, 77, 83, 84, 85, 86, 87]. Gratings with small numbers of elements are interesting because they are less frequency selective, and we show here that the limiting case of a single scattering object, such as a ridge or a groove in a metallic film, can also act as a relatively efficient broadband coupler from freely propagating modes to guided modes [75, 88, 89].

While realistic solar cell designs require arrays of nanostructures to garner large scale absorption enhancements, we begin our design by analyzing mode coupling from single nanostructures. The ideal solar cell nanostructure will both couple incident light efficiently into waveguide modes of a thin cell over a broad wavelength and angular range, and will couple to the modes that deliver the highest absorption into the semiconductor and the least loss into the cladding layers. This chapter

describes the application of the FMSA method discussed in chapter 3 to assess the coupling of individual nanostructures to particular waveguide modes across the solar spectrum and with angle of incidence variation. We apply the method to study the 


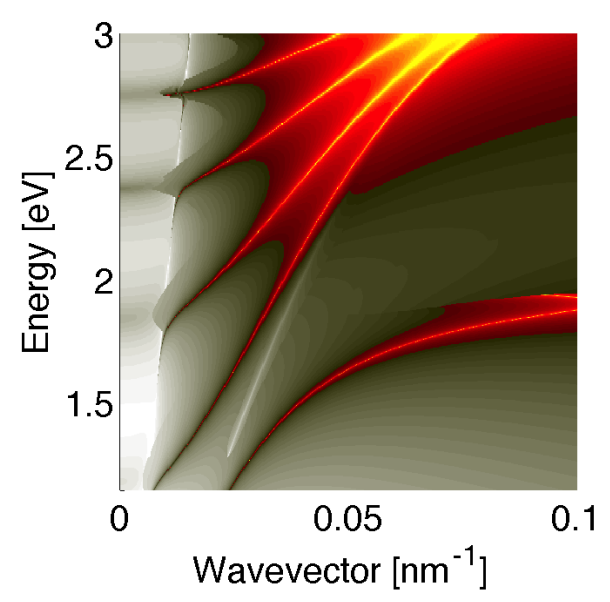

(a) $200 \mathrm{~nm} \mathrm{c-Si}$

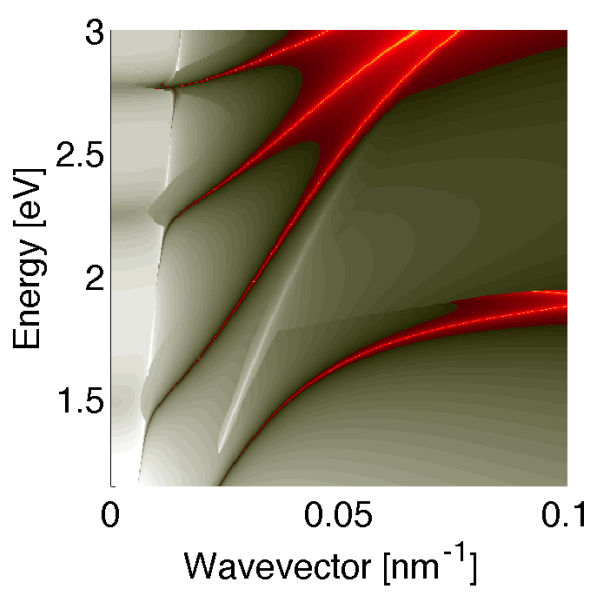

(b) $150 \mathrm{~nm} \mathrm{c-Si}$

Figure 4.1. Dispersion relations for three-layer waveguide: Ag/c$\mathrm{Si} /$ air.

coupling from different nanostructures, and to understand the factors responsible for increased coupling efficiency. In the second part of the chapter, we analyze the absorption enhancements in thin films from single nanostructures of various materials, study the angular dependence of single scatterers, and begin to model the absorption enhancements from arrays of objects.

\subsection{Resonances Affecting Incoupling to Modes}

We chose to analyze a simple system that resembles an ultrathin film Si solar cell: a 150 - $200 \mathrm{~nm}$ thick film of c-Si on an Ag film. While this structure does not include standard cell components such as an antireflective coating, it is a useful simplified solar cell for studying and analyzing mode coupling across the solar spectrum. The aim is to make the structure act as a waveguide, where modes propagate within the c-Si core and Ag and air serve as the cladding layers. We chose these thicknesses as an example case (rather than $500 \mathrm{~nm}$ or $1 \mu \mathrm{m}$ thick films) because they support one SPP mode and one or two photonic modes across the red portion of the spectrum, making it a particularly simple test case for the FMSA method. Figure 4.1 shows calculated dispersion relations for these two thicknesses of c-Si [90]. The number of 


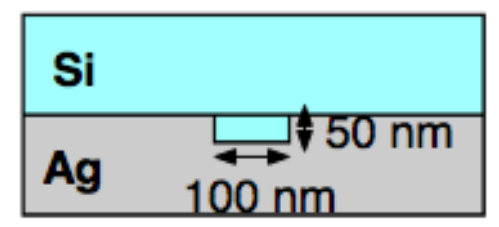

Figure 4.2. Schematic of groove cut into Ag back contact on thin film of c-Si.

supported modes naturally depends on the thickness. Near the band edge of c-Si $(1100 \mathrm{~nm}$, or $1.1 \mathrm{eV}$ ), the $150 \mathrm{~nm}$ thick film supports only the SPP mode and the photonic mode is pushed into cutoff. At other wavelengths in the red portion of the spectrum a small number of modes are supported.

The first scattering object we consider is a subwavelength groove, $100 \mathrm{~nm}$ wide by $50 \mathrm{~nm}$ deep, cut into the $\mathrm{Ag}$ (Fig. 4.2). Since we are interested in the broadband incoupling response for each nanostructure, we tested the groove across a range of incident wavelengths matching the region of the solar spectrum where c-Si exhibits significant spectral response, from 400 to $1200 \mathrm{~nm}$. Figures 4.3(a) and 4.3(d) show typical examples of the incoupling cross sections $\left(\sigma_{i n c}\right)$ for SPP modes and photonic modes calculated using the FMSA procedure described in Chapter 3. Figure 4.3(a) is for a device with a $200 \mathrm{~nm}$ thick Si layer, and Figure 4.3(d) is for a $150 \mathrm{~nm}$ thick layer; all other conditions are identical. Both structures exhibit several strong peaks in $\sigma_{i n c}$. As expected, the incoupling to propagating modes is stronger when the absorption in c-Si weak, i.e. near the band edge. Since these particles are on the back, scattering will also only occur at wavelengths where the c-Si film is not optically thick.

We sought to identify the characteristics that determine the unique shape of the spectrum of $\sigma_{i n c}$. We find that the modal incoupling efficiencies of these structures across the investigated spectral range can be described by the convolution of three different physical resonances in the device. First, the interference within the thin film leads to a resonant Fabry-Perot cavity effect within the film at various wavelengths, depending on the film thickness [17]. The multiple reflections at resonant frequencies in the film leads to stronger interaction of incident light with the scattering object, and to increased incoupling into propagating modes. Second, at the SPP resonance 

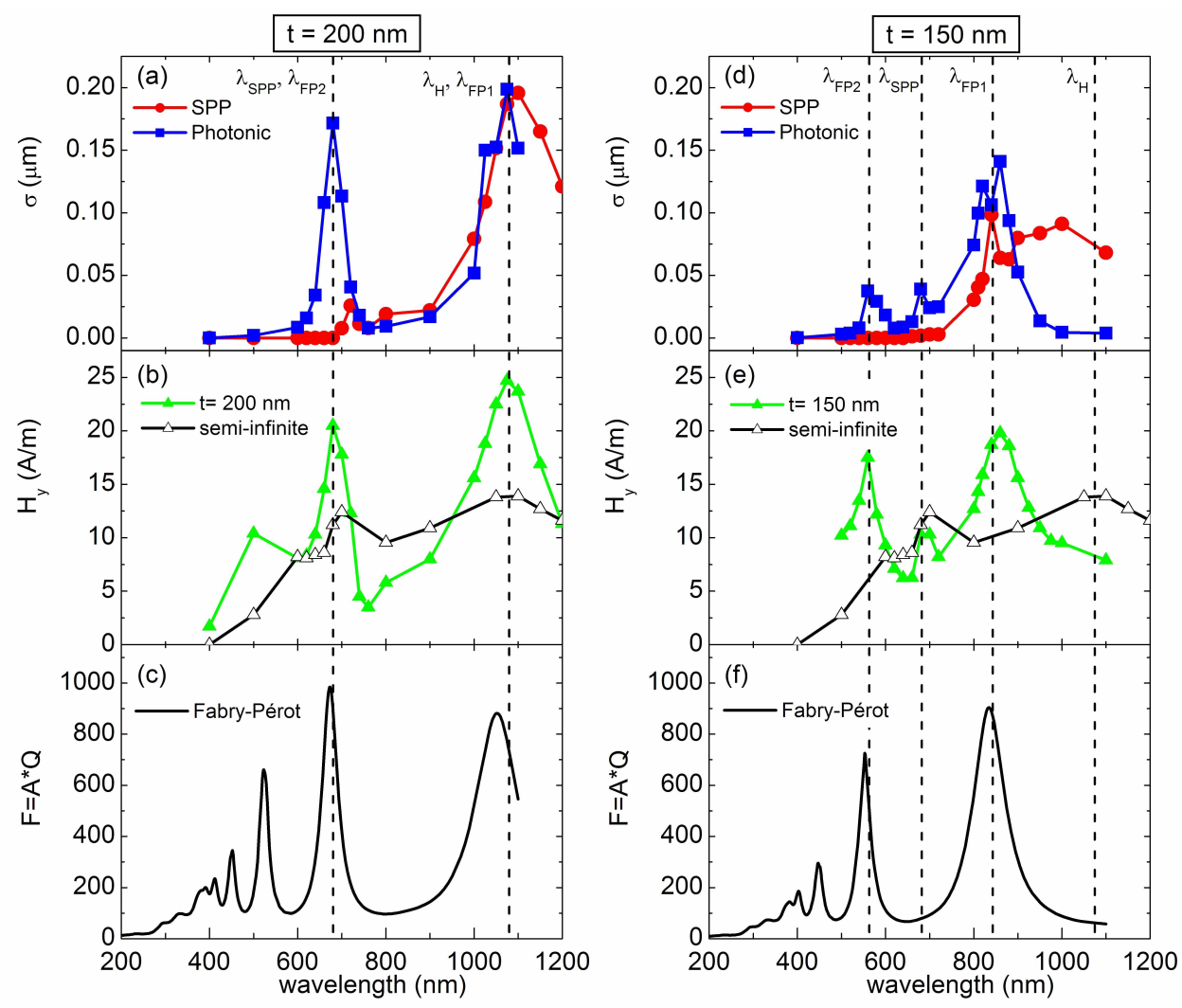

Figure 4.3. Calculated incoupling cross sections $(\sigma), H_{y}$ field magnitudes, and quality factor for the Fabry-Perot cavity in the Si film. Left column for $200 \mathrm{~nm}$ thick film Si, right column for $150 \mathrm{~nm}$ thick film of $\mathrm{Si}$, both on $\mathrm{Ag}$ with a $100 \mathrm{~nm}$ wide by $50 \mathrm{~nm}$ deep groove cut into the Ag at the interface. Panels (a) and (d) show the incoupling cross sections for each mode across the spectral range where Si shows significant response. Panels (b) and (e) show the $H_{y}$ field magnitude measured at the center of the groove mouth for each slab (green) and a semi-infinite layer of $\mathrm{Si}$ on $\mathrm{Ag}$ (black line). Panels (c) and (f) show the factor $F=A \times Q$, where $A$ is the absorbance in the thin film and $Q$ is the quality factor for a cavity of thickness $t$ on Ag, without a groove. 
frequency the high local fields near the scatterer lead to increased incoupling into the photonic waveguide modes. Recall that for an SPP, $k_{x}=\frac{\omega}{c} \sqrt{\frac{\epsilon_{1} \epsilon_{2}}{\epsilon_{1}+\epsilon+2}}$, and the frequency when $\epsilon_{1}=-\epsilon_{2}$ is the SPP resonance. At this frequency the SPP has low group velocity and therefore is bound to the vicinity of the scattering object, so the high fields lead to increased coupling to the propagating photonic modes. Third, the incident light induces a polarization in the scattering object. For objects much smaller than the incident wavelength, the induced polarization is resonant at modes whose number and strength vary with the scatterer's shape and dimensions $[71,91]$. At these resonant frequencies the local fields near the scattering center are very high, and both the absorption cross section and the scattering cross section of the object will be at maximum. The incoupling ability over the entire spectral range is a combination of these three physical phenomena, and the frequency response of incoupling cross sections can be tuned by appropriate modifications of the layer thicknesses and scatterer geometries.

To analyze the incoupling efficiency across the spectrum, we analyze both the field magnitudes in the scattered simulations, and related structures to vary the properties of specific resonances. At all of the resonant conditions mentioned above the field magnitudes will be high near the scattering object. The incoupling cross sections are large near resonant frequencies where the field of scattering object overlaps with the modes of the waveguide. In Figure 4.3(b) and 4.3(e), we calculate the field magnitudes at the centerpoint of the groove mouth for both finite thickness films and a semi-infinite film of c-Si, in order to separate thin film interference effects leading to resonances from inherent resonant properties of the groove. We see that the calculated incoupling cross sections show peaks that line up with the field magnitudes for the finite thickness slabs, from which we deduce that the field magnitude at the groove is a good indicator of incoupling efficiency.

To further investigate the effects of the film thickness on incoupling efficiency, we consider the interference in the thin film. The film can be modeled as a FabryPerot cavity, where the two mirrors are the Si/Air and Si/Ag interfaces, characterized by reflectivities $R_{1}$ and $R_{2}$, respectively. To quantify the absorption enhancements 
occuring at resonant cavity modes, we define a figure of merit $F=A \times Q$, where $A$ is the thin film absorbance, i.e. the fraction of incident energy that gets absorbed in the film as a result of multiple reflections, and $R$ and $T$ are the thin film reflectance and transmittance.

$$
A(\lambda)=1-R(\lambda)-T(\lambda)
$$

Here $Q$ is the approximate quality factor of a Fabry-Perot cavity with thickness $t$, refractive index $n$, absorption coefficient $\alpha$, and reflectivities $R_{1}$ and $R_{2}$ at the two interfaces, given by the following expression:

$$
Q=4 \pi \frac{t n}{\lambda[1-\exp (-2 \alpha t)]\left(1-R_{1}\right)\left(1-R_{2}\right)}
$$

The term $[1-\exp (-2 \alpha t)]$ is the fraction of energy absorbed in the material in a cavity round trip, and the $1-R_{i}$ factors account for the fractions of energy lost at reflection off each boundary. We calculate $R_{1}$ and $R_{2}$ from $R_{i}=\left|r_{i}\right|^{2}$, where $r_{i}$ is the Fresnel reflection coefficient [17].

We compare the reference structures with Fabry-Perot absorption and the semiinfinite film with nanostructure references to the thin-fil waveguide to better understand the origin of the peaks in the incoupling spectrum. The first peak in Figure $4.3(\mathrm{a})$, at $\lambda=680 \mathrm{~nm}$, is present in $\sigma_{\text {inc,photonic }}$ but is absent in $\sigma_{i n c, S P P}$. From comparison with Figure 4.3(b) and Figure 4.3(c), we see that this point lines up with a maximum in the $H_{y}$ field in both the slab and the semi-infinite film, as well as with one of the resonant frequencies of the $200 \mathrm{~nm}$ thick c-Si slab. We identify this peak as a product of both the SPP resonance and the Fabry-Perot resonance of the film. If it were the Fabry-Perot resonance of the film alone, the semi-infinite thickness slab would not have a field maximum at that frequency. The SPP resonance at $\lambda=680$ $\mathrm{nm}$ is also evident from the dispersion relation shown in Fig. 4.1. The convolution of the Fabry-Perot mode and the SPP resonance results in higher incoupling for the photonic mode, as the SPP mode is localized rather than propagating near its resonant frequency. 
In an effort to deconvolute the effect of the Fabry-Perot mode from that of the SPP resonance, we studied a $150 \mathrm{~nm}$ thick film where the Fabry-Perot resonance is shifted to $\lambda=580 \mathrm{~nm}$. We now see two peaks in both $\sigma_{\text {photonic }}$ and $H_{y, 150 \mathrm{~nm}}$ : at the new Fabry-Perot resonance at $\lambda=580 \mathrm{~nm}$ and at the SPP resonance at $\lambda=680 \mathrm{~nm}$. The semi-infinite film shows no field intensity peak at $580 \mathrm{~nm}$, indicating that this incoupling peak is due to the Fabry-Perot resonance alone. As in the $200 \mathrm{~nm}$ thick c-Si film, this incoupling maximum is observable only in the photonic mode, since the SPP mode has short propagation lengths at this frequency. A second Fabry-Perot mode is observed at $\lambda=840 \mathrm{~nm}$, where again the $H_{y, \text { semi-infinite }}$ field is relatively low. Although not shown, we also compared the $E_{x}$ and $E_{z}$ field magnitudes across the spectrum, and at the Fabry-Perot resonant frequencies the in-plane electric field also has a maximum, while the out-of-plane electric field is relatively constant. This is consistent with an out-of-plane resonant Fabry-Perot mode in the slab.

The peak around $\lambda=1100 \mathrm{~nm}$ observed for both film thicknesses is attributed to the magnetic dipole resonance of the groove. The semi-infinite structure, which is exempt from any Fabry-Perot intereference effects has an incoupling peak at $\lambda=1100$ $\mathrm{nm}$, indicating that the enhanced incoupling is not due to the $Q$ of the film. In the $200 \mathrm{~nm}$ thick film, there is significant scattering into both modes, and the position lines up with an additional Fabry-Perot cavity mode, and so we attribute the peak to the convolution of the magnetic dipole with a Fabry-Perot mode in the c-Si film. In the $150 \mathrm{~nm}$ thick film the effect is mainly on the SPP mode; for this reduced thickness the photonic mode approaches its cutoff frequency (Fig. 4.1).

This resonance at $\lambda=1100 \mathrm{~nm}$ can also be affected by the shape of the nanostructure. We further investigate the nature of this maximum in incoupling cross section at $\lambda=1100 \mathrm{~nm}$ by varying the width of the groove from 50 to $300 \mathrm{~nm}$ at fixed depth for the $t=200 \mathrm{~nm}$ case. Figure 4.4(a) shows the incoupling cross section $\sigma$, the "effective" width of the structure, against the physical size of the groove $w$. For the photonic mode, the incoupling cross section increases linearly with size, until it reaches $200 \mathrm{~nm}$ at which point it flattens out. For small grooves the effective scattering cross section is proportional to the physical width $w$ with ratio $\sigma=a \times w$. 
(a)

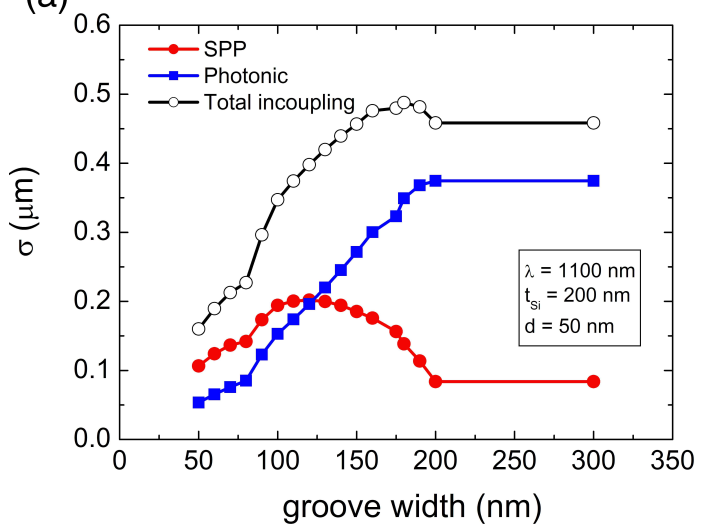

(b)

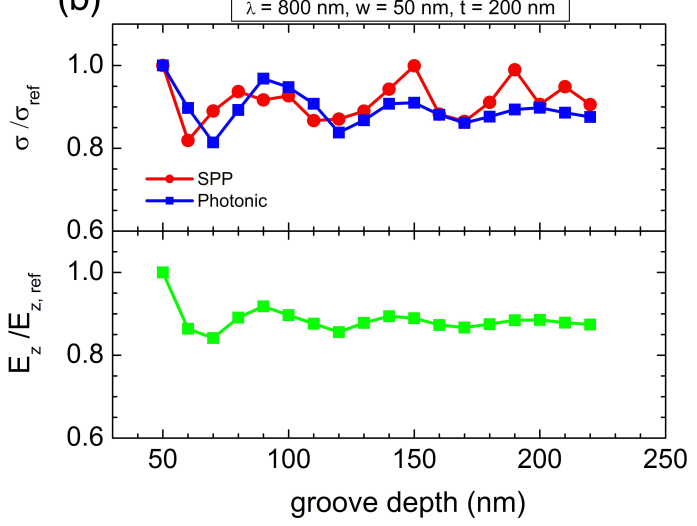

Figure 4.4. (a): Incoupling cross sections for a $50 \mathrm{~nm}$ deep groove with varying physical width $w$, at $\lambda=1100 \mathrm{~nm}$.(b): Incoupling cross sections and field magnitudes for $50 \mathrm{~nm}$ wide groove with varying depth, at $\lambda=800 \mathrm{~nm}$. The top panel shows the photonic and SPP incoupling cross sections, normalized to a reference groove that is 50 $\mathrm{nm}$ wide and $50 \mathrm{~nm}$ deep; the bottom panel shows the magnitude of the $E_{z}$ field, measured at the centerpoint of the groove mouth, and normalized to the $E_{z}$ field for the reference groove.

This relationship breaks down for larger grooves, which behave less like quasistatic dipole scatterers. Above $w=200 \mathrm{~nm}$ the edges of the groove act independently, and there is no further gain from increasing size. The incoupling to SPP modes is strongly affected by this dipole character; the SPP cross section peaks at $w=110$ $\mathrm{nm}$, then declines for larger grooves. Since the number of photonic modes depends on the thickness of the absorbing layer, and for very thin devices only the plasmonic mode will be supported, this confirms that subwavelength scatterers are important for the thinnest devices.

Another type of resonance that could effect the incoupling efficiency of the groove are Fabry-Perot cavity modes inside the groove itself. We probe the effect of this type of resonance by choosing a small width $(w=50 \mathrm{~nm})$ and varying the depth incrementally from 50 to $220 \mathrm{~nm}$ at one incident wavelength $(\lambda=800 \mathrm{~nm})$. In Figure 4.4(b) we show the ratio of the incoupling cross sections, with respect to a reference groove that is $50 \mathrm{~nm}$ deep. The bottom panel shows the ratio of the magnitude of 


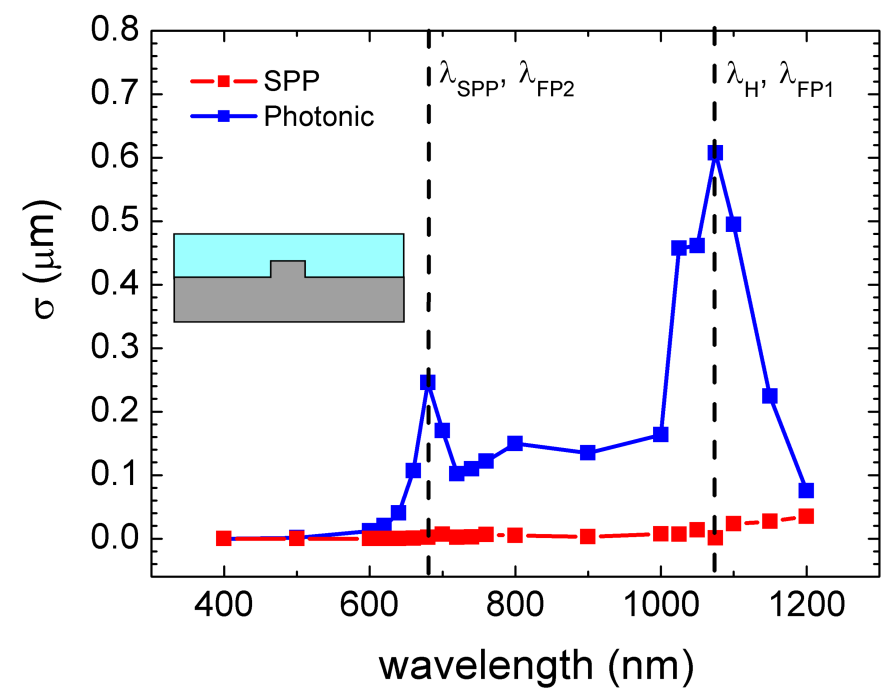

Figure 4.5. Incoupling cross sections for a $100 \mathrm{~nm}$ wide by $50 \mathrm{~nm}$ tall ridge of $\mathrm{Ag}$ in $200 \mathrm{~nm}$ of Si.

the $E_{z}$ field at the groove mouth with respect to this same reference groove. The first interesting detail is that the shallowest groove studied, $d=50 \mathrm{~nm}$, is the most efficient incoupler. As the groove gets deeper, the amplitude of the oscillation decreases, as more energy is absorbed within the groove and the field magnitude is weak at the interface. Most importantly, however, the variation with depth is quite small, and we conclude that while the Fabry-Perot mode in the groove cavity is present, it is not a critical feature of design.

\subsection{Ridges vs. Grooves}

Turning our attention from groove couplers to ridge couplers of the same aspect ratio, the scattering response changes, shown in Figure 4.5. Although the qualitative spectral shape is similar, with peaks at $\lambda=680 \mathrm{~nm}$ and at $\lambda=1100 \mathrm{~nm}$, the incoupling cross sections are much higher for the ridge, and energy is predominantly coupled into photonic modes. For example, at $\lambda=1100 \mathrm{~nm}$, the total incoupling cross section is $\sigma_{\text {inc,groove }}=0.35 \mu \mathrm{m}$, with $\sigma_{\text {inc,photonic }}=0.15 \mu \mathrm{m}$, while for the ridge the total incoupling cross section is $\sigma_{\text {inc,ridge }}=0.52 \mu \mathrm{m}$, with $\sigma_{\text {inc,photonic }}=0.5 \mu \mathrm{m}$. Intuitively 


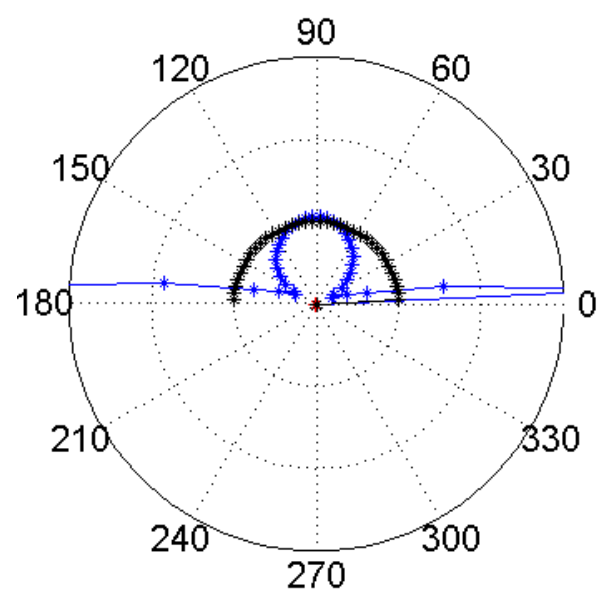

(a) Backscattering from groove.

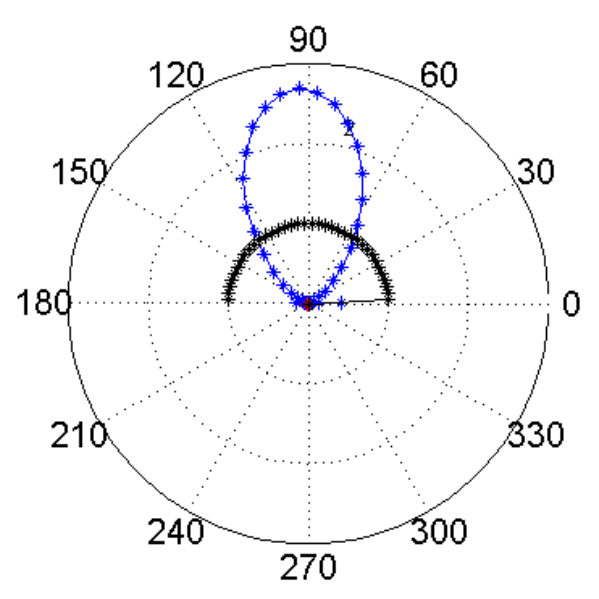

(b) Backscattering from ridge.

Figure 4.6. Angular backscattering from a single nanostructure on an $\mathrm{Ag}$ back contact, with a semi-infinite film of c-Si. The black line is a Lambertian reference.

this is reasonable because the ridge is a polarizable dipole-like scatterer located closer to the middle of the waveguide, whereas the groove is a dipole-like scatterer located in the plane of the metal-semiconductor interface. The position of the effective dipole of each scatterer in the waveguide compared to the field intensity of each mode in the waveguide determines the strength of coupling. This will be explored in more detail in Chapter 5. Although the total incoupling cross section is higher for the ridge, ultrathin devices that do not support photonic modes will be more efficiently served by subwavelength grooves.

Figure 4.6 compares the scattering from a groove and a ridge in a semi-infinite film of c-Si. The black line is a Lambertian scatterer, shown for reference. Both the groove and the ridge scatter like dipoles (as they are subwavelength), but a significant portion of the scattered power from the groove is lateral. The lateral scattering is due to coupling to the surface-bound SPP modes. As these calculations were done with a semi-infinite semiconductor, there are no photonic modes supported, and the ridge appears to scatter most of its power backward rather than into the SPP modes. 


\subsection{Absorption Enhancements from Single Scat- tering Objects}

At this point we have found determining factors for the spectral shape of the incoupling cross sections, but we have not considered whether the energy in each mode is absorbed in the semiconductor or lost to the metal. Light coupled into a propagating mode can be absorbed in either material, but cell efficiency will only be improved when the absorption occurs in the semiconductor, which will be responsible for enhanced carrier generation in the cell. To this end, we use a separate computational method to calculate the absorption fractions in each material, as discussed in Chapter

3. The average time-harmonic electromagnetic power absorbed in a given material depends on the divergence of the Poynting vector, $u_{a}=\nabla \cdot \mathbf{S}$, evaluated only over the region filled with the material of interest [92].

$$
u_{a}=\frac{1}{2} \int\left(\omega \epsilon^{\prime \prime}|\mathbf{E}|^{2}+\omega \mu^{\prime \prime}|\mathbf{H}|^{2}\right) d x d z
$$

where $\omega$ is the frequency of the input wave, $\epsilon^{\prime \prime}$ and $\mu^{\prime \prime}$ are the imaginary parts of the permittivity and permeability, respectively, for the materials in the device, and $|\mathbf{E}|^{2}$ and $|\mathbf{H}|^{2}$ are the magnitudes of the electric and magnetic fields. In our structure, $\mu^{\prime \prime}$ $=0$ for all our materials, and this second term drops out.

For each mode that propagates in the device, some portion of the energy will be absorbed in the semiconductor and some lost to the metal. There may also be localized resonances near the groove that are not coupled into propagating modes, but still lead to local enhanced absorption. Although certain frequencies have much higher incoupling cross sections than others, the scattering efficiency shows relatively broad spectral features, and there may be significant absorption enhancement at nonresonant frequencies as well. Since all realistic solar cell designs will be comprised of arrays of scattering objects, and these absorption enhancements are calculated over a full $10 \mu \mathrm{m}$ region, the enhancements will be low. Nevertheless, an understanding of the fraction of power absorbed in the semiconductor in this simplified case will 
(a)

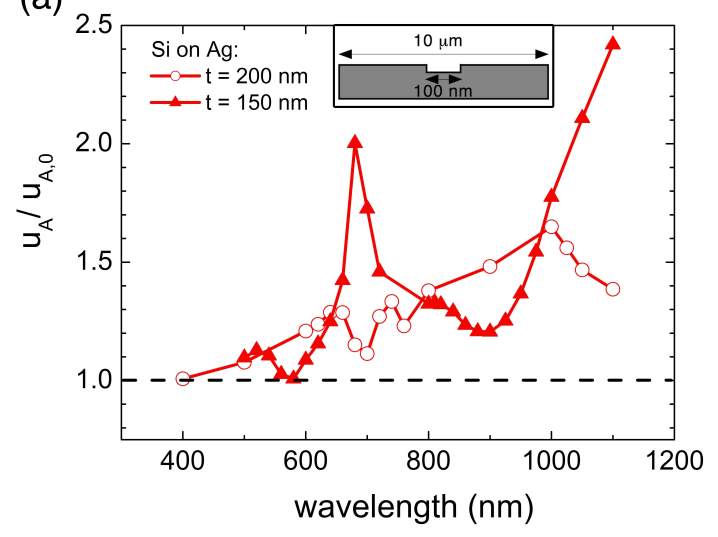

(b)

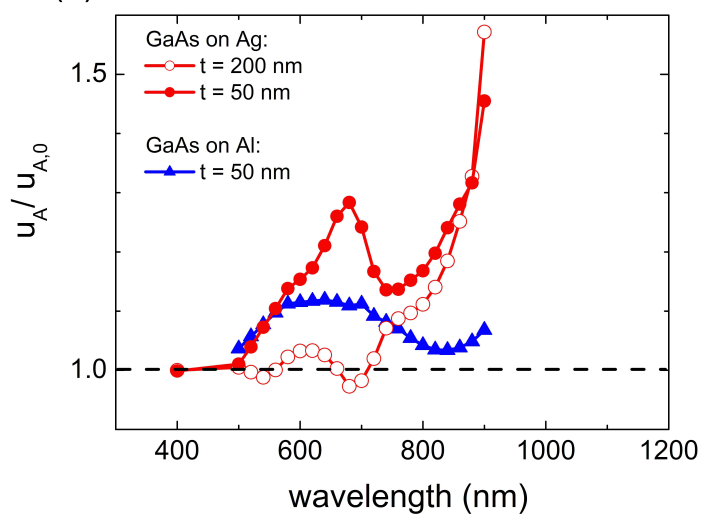

Figure 4.7. Absorption enhancement for a $100 \mathrm{~nm}$ wide by $50 \mathrm{~nm}$ deep groove, relative to the otherwise identical thin film with no groove. Inset shows size of groove compared to the length of the volume considered for enhancement, $10 \mu \mathrm{m}$. Excitation by a Gaussian beam with a $1 \mu \mathrm{m}$ half-width. Panel (a), c-Si on Ag. Panel (b), GaAs on Ag and Al.

inform later design of arrays of nanostructures.

The exact ratio of useful absorption to loss will of course depend on the wavelength, but also on the choice of both semiconductor and metal materials. We define a figure of merit for absorption enhancement, the ratio of absorption in the semiconductor with a groove, $u_{a}$, to the absorption in a identical film without a groove, $u_{a, 0}$. This will cancel out absorption enhancements due purely to the Fabry-Perot resonances of the film, but will preserve the effects of the groove and the propagating modes.

Figure 4.7 shows this absorption enhancement ratio for different materials combinations, in particular for Si (Fig. 4.7(a) and GaAs (4.7(b)). The absorption enhancements are calculated for the entire simulation volume, a $10 \mu \mathrm{m} \times t$ area with $t$ the film thickness, and the groove at the center. The incident beam is a $1 \mu \mathrm{m}$ half-width Gaussian beam.

Looking at Figure 4.7 in more detail, we see that, with the exception of a few data points in the $200 \mathrm{~nm}$ of GaAs, the absorption enhancement ratio is always greater than 1. This demonstrates that the groove has significant broadband response and is not only effective at the resonant frequencies. The dips in both the Si and GaAs profiles 
correspond to the wavelengths of Fabry-Perot resonances. At these resonances, $u_{a, 0}$ is also large and so the effect of the groove is relatively small. In general, we see the strongest absorption enhancement for optically thin layers. Optically dense layers such as the $200 \mathrm{~nm}$ of GaAs absorb a significant fraction of the light before it scatters, and thus have minimal interaction with the scattering nanostructure.

For the $200 \mathrm{~nm}$ thick layer of c-Si in Fig. 4.7(a), the incoupling generally increases with increasing wavelength, except at the frequencies near Fabry-Perot resonances. Near the band edge where there is very little $\mathrm{Si}$ absorption and propagation lengths are long we see the most enhancement over the $10 \mu \mathrm{m}$ area, as well as a peak at the dipole resonance of the groove. For the $150 \mathrm{~nm}$ thick film where the Fabry-Perot resonance is shifted to $\lambda=580 \mathrm{~nm}$, we clearly observe the enhancement at resonance and at the band edge, reaching an enhancement factor of 2.5 at $1100 \mathrm{~nm}$.

The GaAs response is somewhat flatter than the c-Si response, reaching a high of 1.6 near the band edge at $\lambda=880 \mathrm{~nm}$. This is not surprising, since GaAs is a direct bandgap semiconductor and already a strong absorber. In this case the semiconductor absorber layer can be made much thinner, and here we show calculations for a $50 \mathrm{~nm}$ thick layer on both $\mathrm{Ag}$ and $\mathrm{Al}$. The $200 \mathrm{~nm}$ layer shows a relatively small enhancement, except near the band edge where absorption is low. The dips at $\lambda=540$ $\mathrm{nm}$ and $\lambda=680 \mathrm{~nm}$ match the Fabry-Perot film resonances for GaAs. For the $50 \mathrm{~nm}$ film on $\mathrm{Ag}$, the enhancement is much greater, with a strong peak at SPP resonance and the Fabry-Perot dip shifted to $\lambda=760 \mathrm{~nm}$. For the same thickness layer on $\mathrm{Al}$, the spectral shape is quite different because the Fabry-Perot resonance of the film is shifted out to longer wavelengths and has little response in the $\lambda=500-800 \mathrm{~nm}$ spectral region. The losses in $\mathrm{Al}$ are higher, however, so the overall enhancement due to semiconductor absorption is much lower. Overall, then, Ag is the preferred back contact over $\mathrm{Al}$ for c-Si and GaAs. A different conclusion is reached for a-Si:H in other types of device designs in Chapter 7. Additonally, while most of the absorption enhancement occurs at resonant frequencies, there is significant broadband response over a large area of the film. 


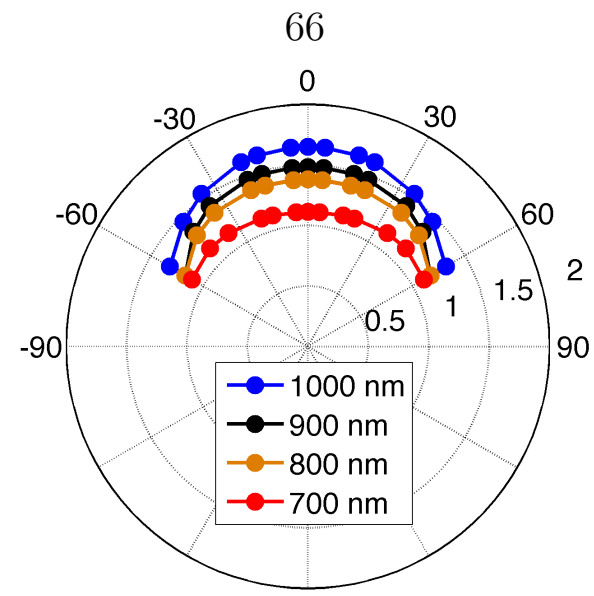

Figure 4.8. Absorption enhancements as a function of angle of incidence for $\lambda=700$ - $1100 \mathrm{~nm}$ incident light. The illumination source is a $1 \mu \mathrm{m}$ half-width Gaussian beam, and the enhancements are calculated over a $10 \mu \mathrm{m}$ area. This enhancement is relative to a control set with no groove.

\subsection{Angle Dependence}

Finally, in Figure 4.8 we consider our other incoupling design goal, angle insensitivity. Figure 4.8 shows the absorption enhancement ratio as a function of angle of incidence for a $100 \mathrm{~nm}$ wide by $50 \mathrm{~nm}$ deep groove in $200 \mathrm{~nm}$ of c-Si, from $0^{\circ}$ to $60^{\circ}$. Since the frequencies of the Fabry-Perot modes change with angle, the absorption enhancement is a more appropriate figure of merit than incoupling cross sections to study the intrinsic behavior of the groove. First, the absorption enhancements at all angles are $\geq 1$, indicating that there are no additional losses due to the modal incoupling at higher angles. We find that at the four frequencies monitored, in the $\lambda=700-1000$ $\mathrm{nm}$ region where there is significant power coupled into SPP modes, the enhancements are quite isotropic, confirming that single subwavelength grooves can act as broadangle incouplers.

\subsection{Arrays of Scattering Elements}

All realistic solar cell designs will consist of arrays of scatterers. However, as more scatterers are incorporated with close spacings, coupling to guided modes will become 


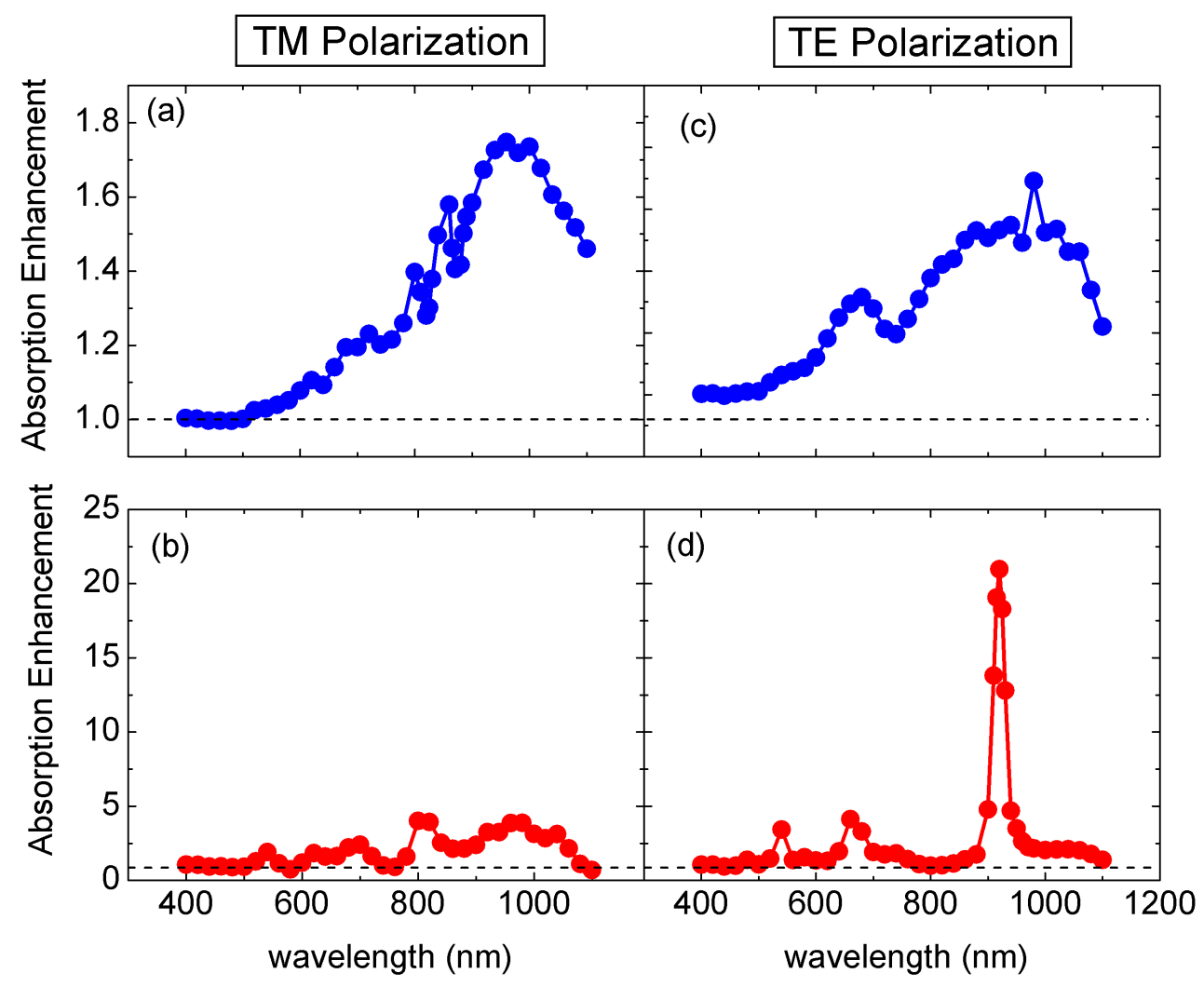

Figure 4.9. Calculated absorption enhancements for nanostructured c-Si cells with an Ag back contact and an antireflection coating. In the top row the scatterers are separated by $6 \mu \mathrm{m}$ pitch, and in the bottom by $300 \mathrm{~nm}$. The dashed line is at an absorption enhancement $=1$, i.e. the case where the absorption is the same as in the planar reference structure. 
more wavelength and angle dependent. We analyze a simple structure here to show the effect of increasing the number of scatterers with close pitch. We also expand our model to include antireflective (AR) coatings and consider the effects of polarization on the observed enhancements.

The simulated device consists of $200 \mathrm{~nm}$ of c-Si on $300 \mathrm{~nm}$ of Ag, with a $60 \mathrm{~nm}$ AR coating on top of the c-Si. The AR coating has the refractive index of $\mathrm{TiO}_{2}$, but is lossless. The basic scattering object considered is a $100 \mathrm{~nm}$ wide by $50 \mathrm{~nm}$ tall metallic ridge off the back of the cell into the semiconductor. These ridges are spaced either widely enough to act independently $(6 \mu \mathrm{m})$ or closely together $(300 \mathrm{~nm})$. The absorption enhancement is defined relative to a planar slab of $200 \mathrm{~nm}$ of $\mathrm{Si}$ on Ag. Our source is a normally incident plane wave, with periodic boundary conditions on the simulation and we study the range of incident wavelengths from 400 to $1100 \mathrm{~nm}$ where c-Si is strongly absorbing. As our simulations are 2D, the ridge is assumed to be semi-infinite along the direction of the grating. The enhancement factor is the ratio of the total absorption in the semiconductor with nanostructures cut into the c-Si to the total absorption in a planar film. Of course the amount of c-Si in the nanostructured device is reduced relative to the control device, but we show here that the enhanced absorption benefits from nanostructuring outweigh the losses from the slight material reduction.

Figure 4.9 shows the calculated absorption enhancements for two different spacings of ridges under both polarizations. Figures 4.9(a) and 4.9(c) show the case where the ridges are sparsely spaced, at $6 \mu \mathrm{m}$, and Figs. 4.9(b) and 4.9(d) show the case where they are close together, at $300 \mathrm{~nm}$ pitch. The dotted line in Figure 4.9 is at absorption enhancement of 1 , which corresponds to the case where the nanostructured device has the same total absorption as the planar reference structure. Note that the y-axes are different; the sparse pitch axis runs to 2.0 (double the absorption of the planar cell), whereas the grating absorption enhancement runs an order of magnitude higher, to 23.

Comparing the top row (6 $\mu \mathrm{m}$ spacing) to the bottom (300 $\mathrm{nm}$ spacing) for either polarization shows the trend we expect: gratings with a close pitch are more sensitive 


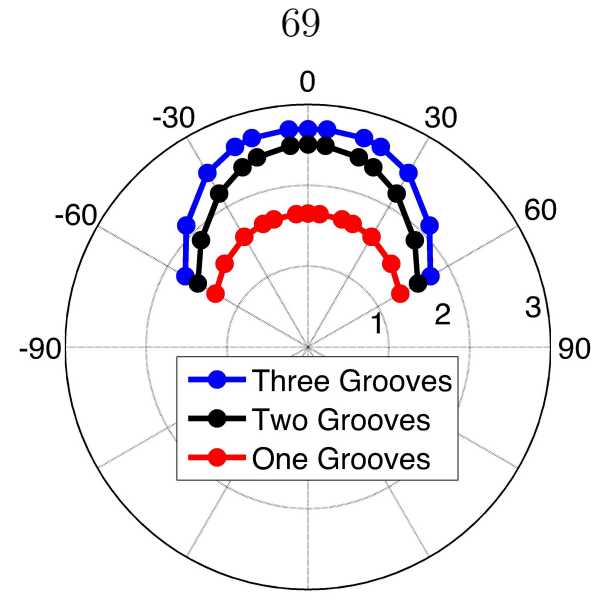

Figure 4.10. Solar spectrum weighted absorption enhancement in $200 \mathrm{~nm}$ of c-Si with varying angle of incidence and increasing number of grooves spaced by $300 \mathrm{~nm}$.

to wavelength, with narrower spectral features and wider variation in enhancement across the spectral range. Recall that for TM polarized light incident on our structure, SPPs can be excited, whereas for TE polarized incident light they cannot. In all four cases the enhancements are largest in the weakly absorbing regime of the spectrum, with a broad resonance centered around $\lambda=1000 \mathrm{~nm}$ that we attribute to the magnetic dipole resonance of the ridge. Notably in the sparse cases, although there are regions that show very little enhancement $(\lambda=400-500 \mathrm{~nm})$, the absorption enhancement factors are always above 1 . The enhancements reach up to $80 \%$ (a factor of 1.8) for the TM case and 70\% (1.7) for the TE case.

Many of the features from the $6 \mu \mathrm{m}$ pitch are exaggerated in the denser system, although new features are also present. The magnitude of the variation is clearly larger for gratings, and there are some wavelengths where the enhancements are well below one. Unsurprisingly, when the enhancements are averaged over the weighted solar spectrum the gratings on average perform better than the $6 \mu \mathrm{m}$ pitch structures. In the TM case, the $300 \mathrm{~nm}$ pitch grating increases absorption by $22 \%$, whereas the $6 \mu \mathrm{m}$ pitch structure increases absorption by $6 \%$. In the TE case the $300 \mathrm{~nm}$ pitch grating increases absorption by $50 \%$, and the $6 \mu \mathrm{m}$ pitch structure by $8 \%$. Higher integrated enhancements could be obtained by further optimizing the geometry and pitch of these structures to regions of the solar spectrum with significant power. 
Finally, we evaluate the increasing angular sensitivity of arrays. Figure 4.10 shows solar spectrum-weighted absorption enhancement with increasing number of grooves, calculated as earlier over a $10 \mu \mathrm{m}$ area with perfectly matched boundary conditions. Since these are isolated arrays, the overall enhancement is significantly smaller than those discussed in Fig. 4.9. The single groove case here is isotropic, but has relatively low absorption enhancements compared to the 2 and 3 groove cases, which also become more angle selective. Nevertheless, small arrangements of subwavelength scatterers are surprisingly angle-insensitive.

\subsection{Conclusions}

In conclusion, we have shown that single subwavelength scattering objects on the metallic back surface of a thin film solar cell can enhance the absorption in the semiconductor over large areas, observing up to $2.5 \mathrm{x}$ enhancement at the longest wavelengths investigated. We find that the spectral scattering response depends on the geometric factors of the object, the thickness of the absorbing film, and the choice of materials, and quantitatively show how both the incoupling cross section and semiconductor absorption can be tuned and enhanced throughout the visible spectrum. The choice of SPP vs. photonic modes depends on other considerations, such as the thickness tolerance and diffusion length of the carriers. For materials with short diffusion lengths, groove structures can couple efficiently to SPP modes. For higher quality materials, however, the absorption benefits from photonic modes are significant, and slightly thicker films may show more improvement. In either case, these structures give insight into the design of nanostructures for broadband and angle-insensitive waveguide-mode based absorption. In the next chapter we will explore the connection between waveguide modes and absorption in more detail. 


\section{Chapter 5}

\section{How Much Do Guided Modes Enhance Absorption in Thin Semiconductor}

\section{Volumes?}

\section{$5.1 \quad$ Introduction}

While the theoretical limits to the efficiency of single and multijunction solar cells have long been understood [93, 94, 95] fabricating cells that approach these limits remains a challenge. Ideally, a single-junction cell absorbs all above-gap photons incident on it and all photogenerated charge carriers are collected, up to the limit imposed by thermodynamics. In practice, harvesting photons in the weakly absorbing spectral range near the band edge requires thick layers, driving up cost, energy of manufacture, and material quality requirements. Surface texturing of thick absorber layers increases the path length of weakly absorbed light inside both indirect and direct gap semiconductors and is routinely employed to enhance absorption in crystalline silicon [96] amorphous silicon [97] and other cells. Much current research, however, focuses on enhancing absorption in thin absorber layers (less than a few micrometers), for which texturing with features larger than the wavelength is not possible.

Thin cells have many potential advantages. Besides requiring less material, thin cells tolerate materials with shorter diffusion lengths, which opens up new classes of materials for thin-film photovoltaics. Furthermore, higher photocarrier concentration in thin absorber layers can potentially boost the open-circuit voltage, thereby increasing efficiency. Stuart and Hall [57, 98, 99] demonstrated up to $18 \times$ absorp- 
tion enhancement near $\lambda=800 \mathrm{~nm}$ in a silicon photodetector caused by a layer of subwavelength silver particles spaced from the front surface of the semiconductor by a thin dielectric layer. A similar approach has been used on amorphous silicon [47] and GaAs [48] absorbers. Recently, Catchpole and co-workers have found similar enhancements in silicon-on-insulator solar cells using the same approach: thin silver films are annealed to produce nanoparticles on the front surface $[46,100]$. Above the plasmon resonance, however, these particles exhibit a Fano resonance that serves to reduce absorption in the blue, indicating that the particles would be more beneficial decorating the back surface [101].

A structured metal layer at the back of the absorber represents another approach to persuading light to propagate parallel to the surface. Using two-dimensional finitedifference-time-domain (FDTD) simulations, Ferry et al. have observed absorption enhancement from coupling to waveguide modes caused by subwavelength metallic grooves and ridges in thin layers of silicon and GaAs backed with silver [67]. Furthermore, for a range of ridge heights and widths, the scattering pattern of isolated structures is essentially dipolar [71] and the scattering cross section of ridges may be several times their geometric cross section. These results motivate the current study in which we use point dipoles to model ridges or other subwavelength objects with high dielectric contrast embedded in the semiconducting absorber layer. We neglect, to a first approximation, their effect on the guided modes of the multilayer structure, which may otherwise include absorbing layers, antireflection layers, in addition to the optically thick metallic substrate layer. The dipoles scatter light into both radiative modes (within the escape cone) and guided modes, including the surface plasmon polariton (SPP) mode localized at the metal/semiconductor interface. Within this simplified semi-analytical model, we seek to understand

- the fraction of power in a guided mode that is absorbed in the semiconductor;

- where the dipoles should be positioned for maximum absorption in the semiconductor; and

- the greatest absorption enhancement possible through this scattering mecha- 
nism.

\subsection{Method}

Our approach proceeds in four steps. First, we find the fraction of the power in each guided mode of a given multilayer structure that is absorbed in each layer. After all, this method will be useful only if the scattering into guided modes serves to enhance absorption in the semiconductor, not the metallic substrate. Second, for a given position and orientation of an embedded dipole we compute the fraction of light scattered by the dipole that is coupled into both radiating modes and guided modes, taking proper account of the reflections at all the interfaces of the multilayer structure. That is, we neglect any direct coupling between dipoles. Although the plasmon resonance frequency of metal nanoparticles is strongly affected by another metal nanoparticle within a few nanometers, we explore here separations of hundreds of nanometers. At these distances, direct dipole-dipole coupling should be negligible. Third, power scattered into each mode is propagated (incoherently) from the scattering dipole to the nearest neighbor dipole a distance $L$ away, keeping track of the portion absorbed in each layer. This dipole then scatters a portion of the power incident on it, leaving the remainder in the various guided modes. The infinite series of subsequent scattering events in this diffusion process is summed to yield the total absorption in each layer, as well as the portion that escapes into the incident medium, for unit intensity scattered by the first dipole. This approach is surely incorrect at any particular wavelength, for which well-defined phase relationships exist between the various scattered waves. However, for broadband excitation in structures with a variety of separations between dipoles, it should capture the average aggregate behavior. Fourth, we estimate the fraction of light incident on the structure at given polarization and angle that scatters in the first step of the diffusion process, from which it is possible to calculate the total absorption in each layer. 


\subsubsection{Absorption in Guided Modes}

We assume that the structure may be modeled as having plane, parallel layers of homogeneous and isotropic dielectric properties. That is, we will assume that, for purposes of finding the guided modes of the multilayer structure, we may neglect the ridges or other intrusions that function as dipole scattering sources. We may then solve for the bound modes at a given frequency by requiring the fields to decay exponentially in the incident medium and the substrate.

A monochromatic wave of angular frequency $\omega$ inside a homogeneous nonmagnetic medium of (complex) refractive index $n$ satisfies the Helmholtz equation,

$$
\left(\nabla^{2}+n^{2} \widetilde{\omega}^{2}\right) \mathbf{E}(\mathbf{r}, t)=0
$$

where $\widetilde{\omega}=\omega / c$ and $c$ is the speed of light in vacuum. Plane wave solutions to this equation take the form

$$
\mathbf{E}(r, t)=\mathbf{E} \exp [i(\mathbf{k} \cdot \mathbf{r}-\omega t)]
$$

in which the wave vector $\mathbf{k} \equiv \mathbf{u} \widetilde{\omega}$ must satisfy the condition

$$
\mathbf{u} \cdot \mathbf{u}=n^{2}
$$

Boundary conditions at each interface may be satisfied only if $u_{x}$ is common to

all layers, which is Snell's law. In a layer of refractive index $n_{j}$, Equation 5.3 gives the magnitude of the normal component of the wave vector:

$$
u_{z, j}=\left(n_{j}^{2}-u_{x}^{2}\right)^{1 / 2}
$$

where we take the root having positive imaginary component, and the two linearly independent plane-wave solutions to Equation 5.1 are

$$
\mathbf{E}_{ \pm}(\mathbf{r}, t)=\mathbf{E}_{ \pm} \exp \left[i \widetilde{\omega}\left(u_{x} x \pm u_{z} z\right)-i \omega t\right]
$$




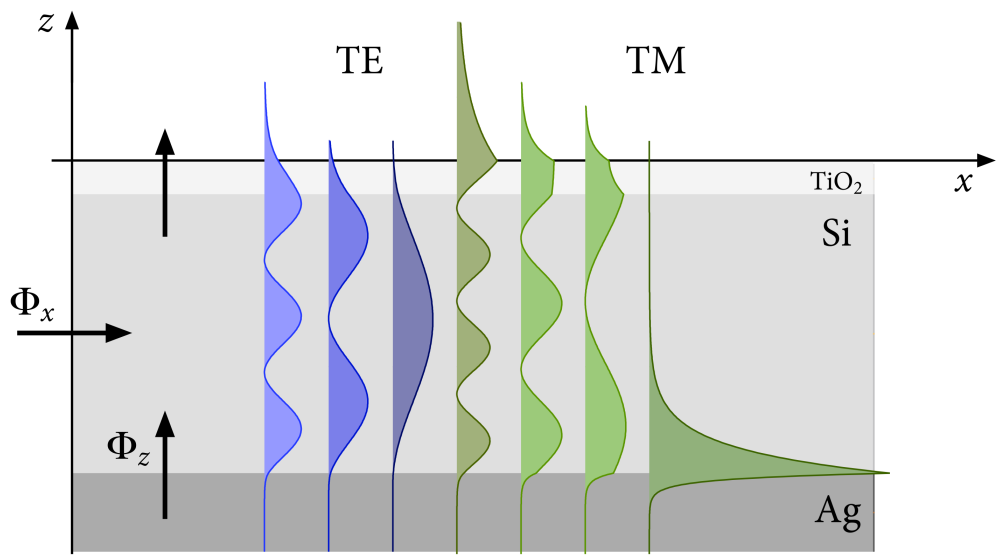

Figure 5.1. A multilayer structure with an antireflection-coated silicon absorbing layer on top of an optically thick layer of silver. Absorption in each layer is determined by integrating the power flux through the $x=0$ plane and through the constant- $z$ interfaces between layers. The illustrated modes have been calculated for a 500 $\mathrm{nm} \mathrm{Si}$ layer on $\mathrm{Ag}$, with a $56 \mathrm{~nm} \mathrm{TiO}_{2}$ layer on top, at $\lambda=1100 \mathrm{~nm}$.

For real $u_{x}<1$, the wave may propagate in the vacuum outside the multilayer; for real $u_{x}>1$, or if $u_{x}$ has an imaginary component, the mode decays exponentially in the vacuum; if the substrate is absorbing, or if $u_{x}>n_{\text {substrate }}$, then the mode is confined and propagates parallel to the surface. Eigenvalues of $u_{x}$ are found using the standard transfer matrix formalism [80] to compute the fields in the incident medium corresponding to a purely decaying exponential in the substrate, and requiring the exponentially growing field to vanish.

By momentum conservation, a plane wave incident at any angle from vacuum does not couple to guided modes; however, a scatterer in or near the multilayer can launch a guided wave. We would like to know what fraction of the guided wave's power is absorbed in each layer of the structure. As discussed in the appendix, this may be computed by integrating the time-averaged Poynting vector over the boundaries of each layer and the plane at $x=0$. As an example, consider the structure illustrated in Fig. 5.1, which shows a $500 \mathrm{~nm}$ Si layer on an optically thick Ag layer, covered with a $56 \mathrm{~nm}$ antireflection layer of $\mathrm{TiO}_{2}$. The illustrated modes for $\lambda=1100 \mathrm{~nm}$ are shown with increasing $\Re u_{x}$ from left to right, TE modes on the left and TM modes 
on the right. For the SPP mode at the far right, the fraction of power absorbed in the silicon layer is $0.35 \%$, with the remainder absorbed in the silver. Thus, $1100 \mathrm{~nm}$ light coupled into the SPP mode serves almost exclusively to heat the silver layer.

On the other hand, absorption in $\mathrm{Si}$ at this wavelength is quite weak. The fraction of light absorbed in propagating across a $500 \mathrm{~nm}$ layer (in the absence of scatterers) is $1-e^{-\alpha d} \approx \alpha d=0.052 \%$, so that nearly 7 times more energy is absorbed in the silicon layer in the SPP mode than in a single traversal of the layer at normal incidence, provided that the wave does not scatter out of the mode. We call this ratio the absorption enhancement $\eta$ of the mode. Taking the ratio to the absorption of a single pass at normal incidence facilitates a comparison to the ergodic limit, first computed by Yablonovitch and Cody, to estimate absorption enhancement in thick silicon layers with roughened surfaces $[16,102,103]$ The ergodic limit assumes that absorption is very weak $(\alpha d \ll 1)$ and that scattering is sufficient to produce an isotropic photon gas inside the semiconducting slab. Because of the large refractive index of the semiconductor, most of the light incident on the front interface from within the slab is totally internally reflected, so that the average ray makes $2 n^{2}$ traversals of the layer. Furthermore, the average traversal has length $2 d$. In the weak absorption limit, therefore, absorption is $4 n^{2} \alpha d$, which is enhanced by a factor of $4 n^{2}$ with respect to a single pass through the layer at normal incidence, $\alpha d$.

Other modes exhibit significantly greater enhancement. From left to right, the transverse-electric modes (TE or $s$-polarized modes with $\mathbf{E}$ perpendicular to the plane of incidence) have enhancements $\eta$ of 73,166 , and 542, while the transverse magnetic (TM or $p$ ) modes have enhancements of 39,43 , and 76 . That is, $28.3 \%$ of the energy in the fundamental TE mode is absorbed in the silicon layer, despite the very weak absorption coefficient $\left(n_{S i}=3.547+9.14 \times 10^{-5} i\right)$. This enhancement arises because the mode energy is concentrated in the silicon layer, not in the silver substrate, and assumes that the light does not scatter out of the mode. Since absorption is proportional to $\epsilon^{\prime \prime}|\mathbf{E}|^{2}$, where $\epsilon^{\prime \prime}$ is the imaginary part of the dielectric constant, minimizing the field in the metal minimizes Joule losses.

Figure 5.2 shows the absorption enhancement $\eta$ in both TM and TE modes for 


\section{7}
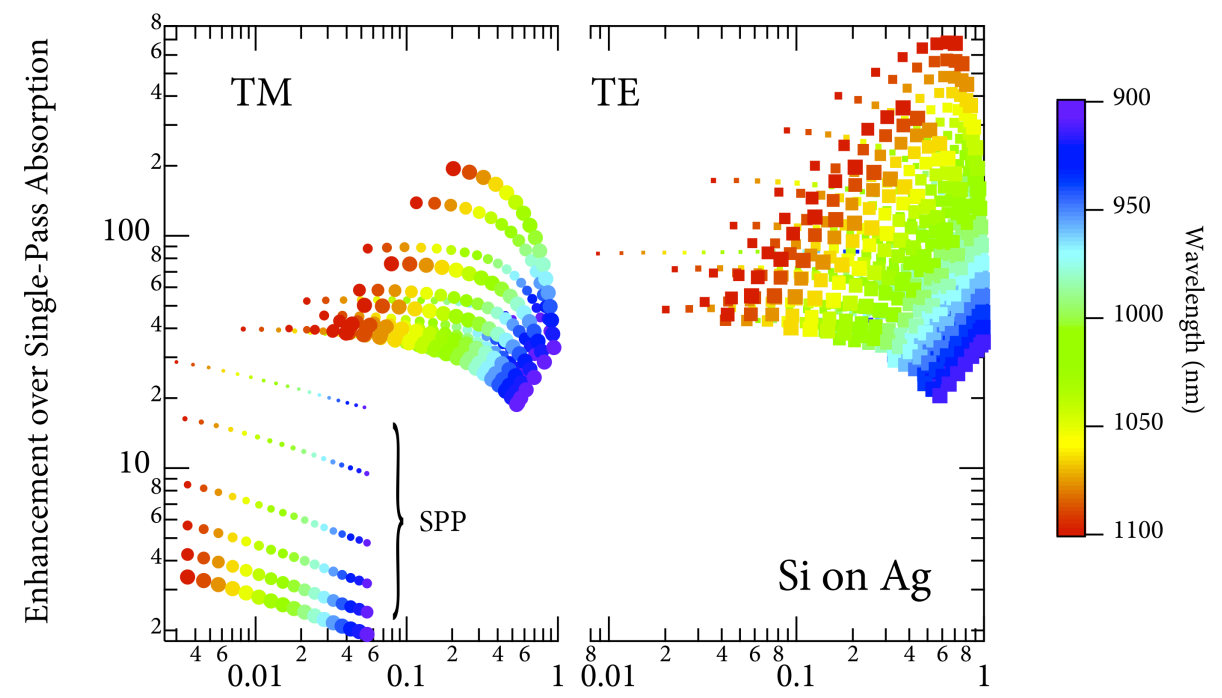

Fraction Absorbed in Semiconductor

Figure 5.2. Absorption enhancement $\eta$ in a silicon layer on silver compared to a single pass at normal incidence for TM modes (left panel) and TE modes (right panel) as a function of the fraction of energy absorbed in the silicon layer. The marker size shows the thickness of the silicon layer from 100 to $1000 \mathrm{~nm}$ and color indicates the wavelength. The SPP mode shows significantly smaller enhancement than other modes, decreasing with increasing layer thickness. For the photonic modes, the absorption enhancement tends to rise with layer thickness. Note that the calculation here assumes that light does not scatter out of the mode.

(bare) silicon layers of various thicknesses and $900 \mathrm{~nm} \leq \lambda \leq 1100 \mathrm{~nm}$. The enhancement is plotted against the fraction absorbed in the silicon layer. The thickness of the silicon layer is represented by the size of the dot, ranging from 100 to 1000 $\mathrm{nm}$; the wavelength is indicated by the dot's color. The TM modes separate into two regions, SPP modes and "photonic" modes, that show distinctly different dependence on layer thickness. The SPP modes lie below enhancements of about 30 and below a fraction absorbed in the silicon of roughly $6 \%$. Since the SPP mode is localized near the $\mathrm{Si} / \mathrm{Ag}$ interface, increasing the thickness of the layer has almost no effect on the fraction absorbed in the silicon; it simply reduces the enhancement compared to a single pass through the layer, since thicker layers absorb more. 
By contrast, for the photonic modes, increasing the layer thickness increases both the fraction absorbed in the semiconductor and the absorption enhancement. These modes satisfy $u_{x}^{\prime}<n_{\mathrm{Si}}^{\prime}$, where primes denote the real part; we call them "photonic" due to their resemblance to the modes of dielectric waveguides. The enhancement grows faster than linearly with the layer thickness because the magnitude of the exponentially decaying field in the metal decreases as the semiconductor thickness increases. At wavelengths near $1 \mu \mathrm{m}$, the fraction absorbed within the semiconductor exceeds 20\%. TE modes exhibit even greater enhancements, as shown in the right panel of 5.2. For layers of roughly $1 \mu \mathrm{m}$ thickness, the enhancement in the most favorable fundamental TE mode exceeds 600. This is more than 12 times better than the ergodic limit, although the ergodic limit includes scattering out of guided modes, of course. As in the ergodic case, large values of $\eta$ arise from very long propagation distances in the silicon layer. As an example, the fundamental TE mode at $\lambda=1100 \mathrm{~nm}$ for a $1 \mu \mathrm{m}$ Si layer on $\mathrm{Ag}$ has $u_{x}=3.509+1.31 \times 10^{-4} 1$, from which we deduce a decay length of $\ell=\lambda / 4 \pi u_{x}^{\prime \prime}=670 \mu \mathrm{m}$.

\subsubsection{Dipole Scattering}

To model scattering into the various guided modes of multilayer structures, we consider a point dipole embedded in a non-absorbing dielectric layer of the structure (including the incident medium or substrate, if desired). We use a method devised originally by Sipe [104] to investigate modifications to emission from atoms or molecules near a metallic plane. The method expands the dipole field in plane waves and assumes a local dielectric response in the metal, which is satisfied for dipoles at least $10 \mathrm{~nm}$ from the metal [105] and has been applied in various forms to a variety of radiation problems $[40,100,106,107,108]$ If we may neglect nonradiative loss in the dipole, and call the decay rate of the dipole in an infinite slab of the dielectric $\gamma_{\text {free }}$, then the normalized decay rate of the dipole is [108]

$$
\hat{\gamma}=\frac{\gamma}{\gamma_{\text {free }}}=\int_{0}^{\infty} \frac{1}{\mathcal{P}_{\text {free }}} \frac{d \mathcal{P}}{d u_{x}} d u_{x}
$$


where the normalized decay rate density is given by

$$
\begin{aligned}
\frac{1}{\mathcal{P}_{\text {free }}} & \frac{d \mathcal{P}}{d u_{x}}=\frac{3}{2 n^{3}} \Re\left\{\frac{u_{x}}{u_{z}}\left[u_{x}^{2} \tilde{r}_{\perp}^{p} \cos ^{2} \theta_{d}+\frac{\sin ^{2} \theta_{d}}{2}\left(n^{2} \tilde{r}_{\|}^{s}+u_{z}^{2} \tilde{r}_{\|}^{p}\right)\right]\right\} \\
\tilde{r}_{\perp}^{p} & =\frac{\left[1+r_{-}^{p} \exp \left(2 i k_{z} d_{-}\right)\right]\left[1+r_{+}^{p} \exp \left(2 i k_{z} d_{+}\right)\right]}{1-r_{-}^{p} r_{+}^{p} \exp \left(2 i k_{z} d\right)} \\
\tilde{r}_{\|}^{s} & =\frac{\left[1+r_{-}^{s} \exp \left(2 i k_{z} d_{-}\right)\right]\left[1+r_{+}^{s} \exp \left(2 i k_{z} d_{+}\right)\right]}{1-r_{-}^{s} r_{+}^{s} \exp \left(2 i k_{z} d\right)} \\
\tilde{r}_{\|}^{p} & =\frac{\left[1-r_{-}^{p} \exp \left(2 i k_{z} d_{-}\right)\right]\left[1-r_{+}^{p} \exp \left(2 i k_{z} d_{+}\right)\right]}{1-r_{-}^{p} r_{+}^{p} \exp \left(2 i k_{z} d\right)}
\end{aligned}
$$

The dipole's axis makes angle $\theta_{d}$ with respect to the normal and is located in a layer of refractive index $n$ at $d_{-}\left(d_{+}\right)$from the adjacent layer at smaller (greater) $z$, $d=d_{-}+d_{+}$is the layer thickness, and $k_{z}=u_{z} \widetilde{\omega}$ is the normal component of the wave vector in the dipole layer. See the inset in Fig. 5.3. The p-polarized amplitude reflection coefficient for all the layers at $z \geq d$ is denoted $r_{+}^{p}$, and similarly for the other reflection coefficients. For the weakly absorbing region, we use a purely real index for the semiconductor for purposes of computing $\hat{\gamma}$. When the semiconductor absorption is too large to ignore, we surround the dipole with lossless "sliver" layers $10 \mathrm{~nm}$ thick having the same value of $n^{\prime}$ as the semiconductor.

As illustrated in the top panel of Fig. 5.3, the normalized decay rate density at $\lambda=1100 \mathrm{~nm}$ of a $1 \mu \mathrm{m}$ Si layer on Ag exhibits six sharp peaks, corresponding to photonic modes, and one broad peak near $u_{x}=4$, corresponding to the SPP mode. This panel shows coupling for a dipole oriented perpendicularly to the plane of the layers (i.e., for which $\left.\theta_{d}=0\right)$, embedded in the silicon layer at $50 \mathrm{~nm}(200 \mathrm{~nm})$ from the Ag substrate in the solid (dotted) trace. The area under the SPP peak for $d_{-}=50 \mathrm{~nm}$ is 2.2 , meaning that the rate at which this dipole radiates into the SPP mode is 2.2 times its rate of emission into $4 \pi$ steradians in an infinite block of silicon. For $d<100 \mathrm{~nm}$, the SPP mode dominates emission into TM modes for both perpendicular and parallel dipoles. Hence, the field is concentrated in the metal substrate, leading to poor absorption enhancement in the semiconductor. We are not so much interested in the rate as in the fraction of power that goes into each of the 


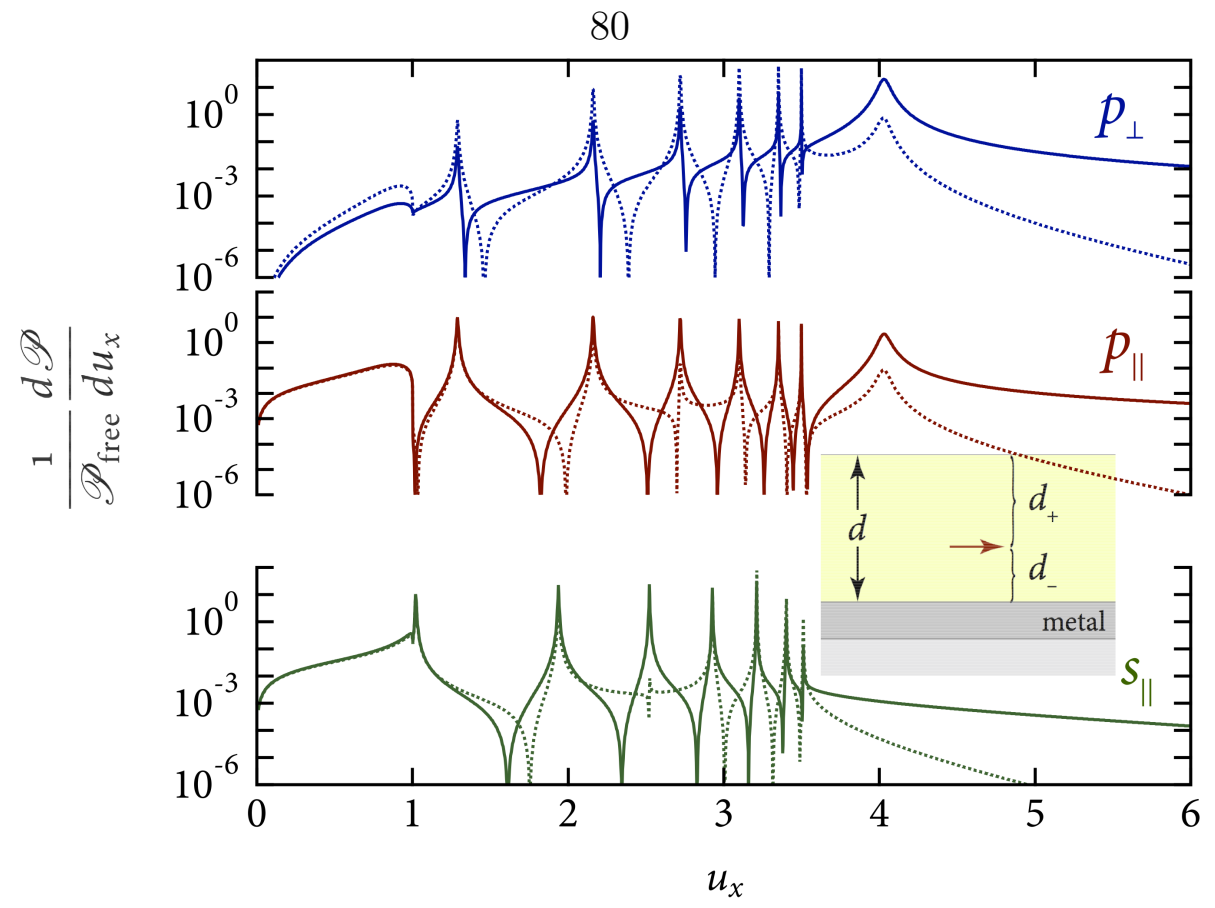

Figure 5.3. Normalized decay rate at $\lambda=1100 \mathrm{~nm}$ for a dipole in a $d=1 \mu \mathrm{m}$ silicon layer a distance $d_{-}=50 \mathrm{~nm}$ from a silver substrate (solid traces) and $200 \mathrm{~nm}$ from the silver layer (dotted traces) as a function of the normalized wave vector component parallel to the surface. The inset illustrates the structure.

modes, including radiative modes, for which $u_{x}<1$. By computing the area under each peak, we find that the fraction of power radiated out of the multilayer for this dipole is $0.04 \%$, while that going into the SPP mode is $95.7 \%$. When the dipole is moved to $d_{-}=200 \mathrm{~nm}$, these fractions change to $0.03 \%$ and $8.3 \%$. In this position, therefore, the great majority of the power goes into photonic modes that exhibit large absorption enhancements.

A dipole oriented parallel to the layers $\left(\theta_{d}=\pi / 2\right)$ couples to both $p$ - and $s$ polarized modes, as illustrated in the lower two panels of Fig. 5.3. To determine the fraction $\rho_{j}$ of emission into mode $j$, we consider both $p_{\|}$and $s_{\|}$together, normalizing against the combined area under both curves. We can now calculate how the scattered light couples to each mode $\left(\rho_{j}\right)$ and the fraction of power in mode $j$ that is absorbed in each layer. Note that $\rho_{0}$ represents the fraction of power that couples to radiating modes. 
81

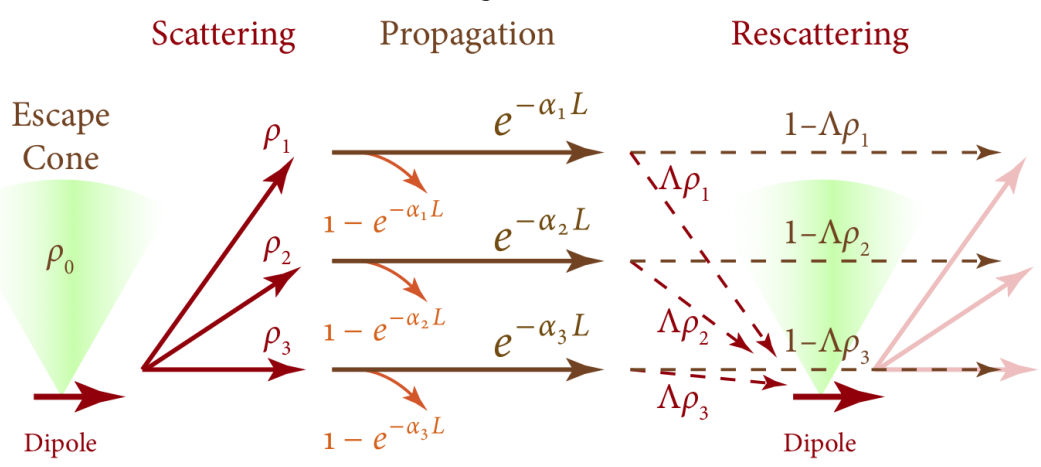

Absorption

Figure 5.4. Diffusion model of light propagation inside the multilayer structure. Light is scattered by the dipole on the left into the escape cone, with fraction $\rho_{0}$, and into guided modes with fractions $\rho_{j}$. Each guided mode propagates a distance $L$ to the next dipole, with loss in each absorbing layer dependent on the mode. At the next dipole, a fraction $\Lambda \rho_{j}$ of the remaining light scatters. Its power is added to the unscattered light in each mode.

\subsubsection{Multiple Scattering}

We now consider the extent to which scattering out of guided modes compromises the very large absorption enhancements of guided modes. Light that scatters from a dipole at distance $d_{-}$from the metal/semiconductor interface into a given guided mode will also efficiently scatter out of that mode on encountering a second dipole that is also at $d_{-}$. We assume that the phase of the light is effectively scrambled on scattering, so that we may treat the propagation in each mode-and the scattering at the next dipole - incoherently. This approach was originally developed by Leblanc et al. [109] to describe the propagation of light in multilayer amorphous silicon structures with rough interfaces, and has been used recently by Catchpole and coworkers to model absorption enhancement from metal nanoparticles on the surface of a multilayer structure [46]. As in the latter work, we take proper account of the discrete modes of multilayer structures, but consider in this work the application of the theory to dipole scattering centers embedded within an absorbing layer of the structure.

The approach is illustrated in Fig. 5.4. Let $\Lambda(\lambda)$ represent the fraction of light 
of wavelength $\lambda$ that is scattered by a given dipole, and $L$ be the typical distance between the dipoles. We consider unit intensity that leaves the dipole, which divides into a fraction $\rho_{0}$ in the escape cone, and $\rho_{j}$ in guided mode $j$. If $f_{\beta j}$ is the fraction of power in mode $j$ that is absorbed in layer $\beta$, and $\alpha_{j}=2 u_{x}^{\prime \prime} \widetilde{\omega}$ is the decay constant of mode $j$, then the power from mode $j$ absorbed in layer $\beta$ between dipoles is

$$
A_{\beta j}^{(0)}=f_{\beta j}\left(1-e^{-\alpha_{j} L}\right) \Sigma_{j}^{(0)}=f_{\beta j} l_{j} \Sigma_{j}^{(0)}
$$

where $\Sigma_{j}^{(0)}=\rho_{j} \Sigma^{(0)}$ is the fraction of the initially scattered flux $\Sigma^{(0)}$ that couples into mode $j$ and $l_{j}$ is the fraction of light in mode $j$ that is absorbed on propagating a distance $L$. The total power absorbed in layer $\beta$ from all guided modes propagating over the distance $L$ is

$$
A_{\beta}^{(0)}=\sum_{j=1}^{N} A_{\beta j}^{(0)}
$$

where the sum is over the $N$ guided modes.

The scattered power in mode $j$ now arrives at the next dipole, where it has probability $\Lambda \rho_{j}$ of scattering. The remaining power, $(1-\Lambda) \rho_{j}$, continues to propagate in mode $j$ past this dipole, a portion being absorbed in each layer until it encounters the next dipole in the next step of the energy diffusion process. Thus, we take the scattering matrix to be

$$
\mu_{i j}=\Lambda \rho_{i} \rho_{j}+\delta_{i j}(1-\Lambda) \rho_{j}
$$

with $\delta_{i j}$ being the Kronecker delta. This expression for the $(N+1) \times(N+1)$ matrix $\mu_{i j}$ is constructed to conserve power and to ignore interference between fields arriving at the dipole from different modes (and the incident wave). If the dipoles are not ideal, but convert a fraction $\hat{g}$ of the scattered power into heat, then $\mu_{i j}$ becomes

$$
\mu_{i j}=\Lambda \rho_{i} \rho_{j}(1-\hat{g})+\delta_{i j}(1-\Lambda) \rho_{j}
$$

The flux that scatters into mode $i$ at the second scattering event is thus 


$$
\Sigma_{i}^{(1)}=\sum_{j=1}^{N}\left(1-l_{j}\right) \mu_{i j} \Sigma_{j}^{(0)}
$$

from which we may compute absorptions $A_{\beta j}^{(1)}=f_{\beta_{j}} l_{j} \Sigma_{j}^{(1)}$, etc.

The infinite series of scattering events may be conveniently summed [109] yielding the total absorption in each layer. Passing to matrix notation, we may express the flux leaving the $n$th dipole as

$$
\left\{\Sigma^{(n)}\right\}=[F]\left\{\Sigma^{(n-1)}\right\}=[F]^{n}\left\{\Sigma^{(0)}\right\}
$$

where $F_{i j}=\mu_{i j}\left(1-l_{j}\right)$. Then the total absorption in each layer is given by

$$
\{A\}=\sum_{n=0}^{\infty}\left\{A^{(n)}\right\}=\sum_{n=0}^{\infty}[f][l][F]^{n}\left\{\Sigma^{(0)}\right\}=[f][l](1-[F])^{-1}\left\{\Sigma^{(0)}\right\}
$$

where $[l]$ has elements $l_{i j}=l_{j} \delta_{i j}$. Note that $[f]$ is an $M \times(N+1)$ matrix, where $M$ is the number of layers, including both the incident medium and the substrate. Thus, for unit flux leaving the dipole, $A_{\beta}$ is the fraction that is absorbed in layer $\beta$.

\subsection{Results}

Figure 5.5 illustrates how the coupling coefficient $\Lambda$ of the dipoles affects absorption enhancement for a $200 \mathrm{~nm}$ Si layer on Ag. The dipoles are assumed to be lossless $(\hat{g}=0)$ and spaced $L=1 \mu \mathrm{m}$ apart. Plotted against the left axis for four different positions of the parallel dipoles with respect to the $\mathrm{Si} / \mathrm{Ag}$ interface is the absorption enhancement $\eta$ of the light that initially scatters as a function of $\Lambda$. In all cases the enhancement decreases monotonically with $\Lambda$, because with increasing $\Lambda$ the probability of scattering out of a guided mode and into the escape cone increases. Since we consider only the light that has already been scattered into guided modes by the first dipole, scattering reduces the net absorption. Of course, without an initial scattering event, none of the light would enter guided modes in the first place. 


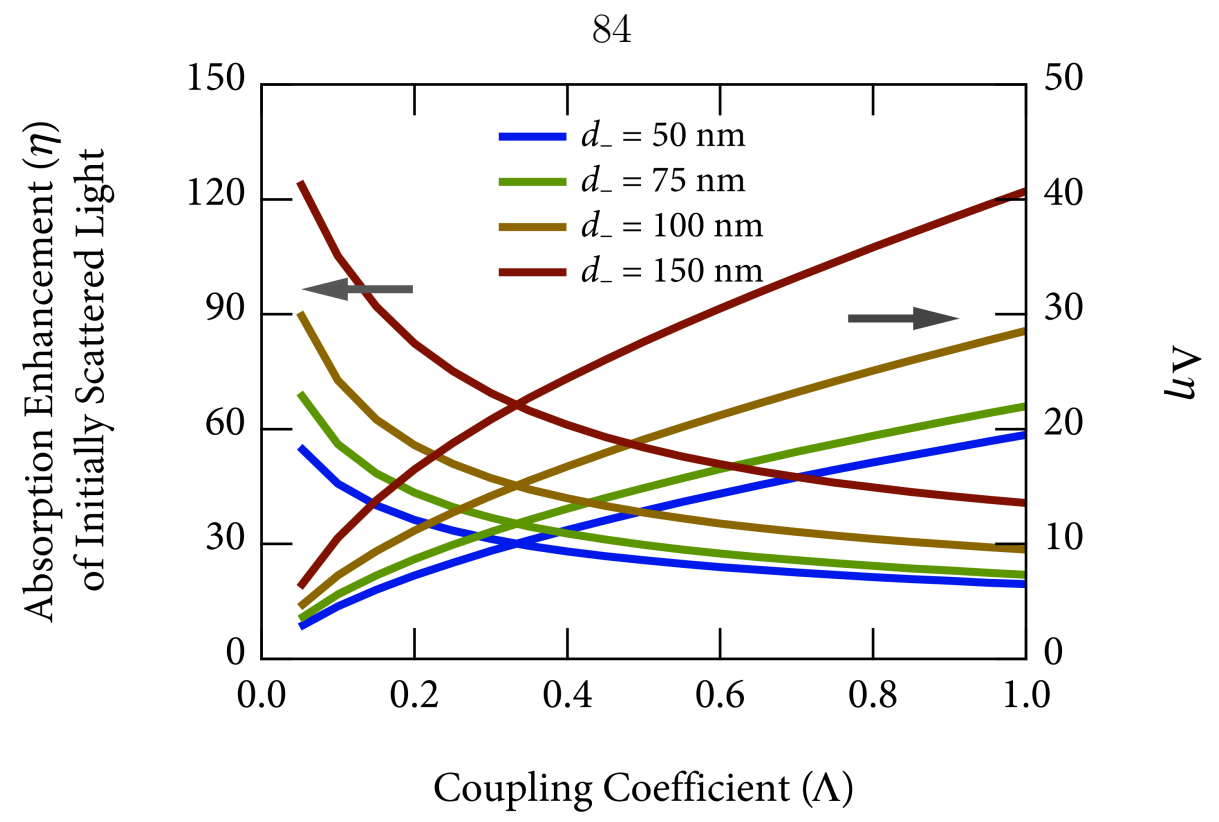

Figure 5.5. Band-edge absorption enhancement ( $\eta$, left axis) of light leaving a dipole a distance $d_{-}$from the $\mathrm{Ag} / \mathrm{Si}$ interface, compared to single-pass absorption for a $200 \mathrm{~nm}$ Si layer on $\mathrm{Ag}$, as a function of coupling coefficient $\Lambda(L=1 \mu \mathrm{m}, \lambda=1100 \mathrm{~nm})$. The product $\Lambda \eta$ (right axis) scales the curves assuming that the initial scattering is also $\Lambda$.

As a crude in-coupling approximation, if we assume that scattering of the incident wave into guided modes is described by the same coupling coefficient $\Lambda$, then $\Lambda \eta$ gives a more realistic estimate of the absorption enhancement. This is plotted for the same four dipole positions against the right axis, and is a monotonically increasing function of $\Lambda$. As is physically reasonable, regardless of dipole position the greatest enhancement occurs for dipoles that scatter all light incident on them.

Estimating more accurately the fraction of normally incident light that scatters on first encountering the dipoles is quite complicated. In the limit of small $\Lambda$, we may treat the dipole scattering as a weak perturbation and use the fields in the absence of the dipoles to estimate the scattering. In this limit, the reflectivity of the structure is approximately unaffected by the dipoles and can be used to estimate the power participating in the diffusion. The field at the dipole consists in the superposition of the forward- and backward-propagating waves, as may be computed with the transfer 
matrix method ([80], $\S 1.6)$. The strong-coupling limit is more interesting, however. With increasing scattering, the fields inside the multilayer will be perturbed in a complicated way away from their values in the absence of scattering.

In the limit of perfect scattering, the forward-going wave is completely eliminated and the situation becomes simple once again. In this case the reflectivity of a thin slab of lossy dielectric becomes that of an infinitely thick slab, $R_{\infty}=\left|\frac{n-1}{n+1}\right|^{2}$, and the field at the dipole located $d_{+}$below the front interface is

$$
E\left(d_{+}\right)=E_{0} t e^{i \tilde{\omega} n d_{+}}
$$

where $n$ is the complex index of refraction of the dielectric and $t=\frac{2}{n+1}$ is the transmission coefficient of the air/dielectric interface. For a bare $200 \mathrm{~nm}$ slab of Si on Ag, this lowers the normal reflectivity at the band edge from $89 \%$ to $31 \%$. An effective antireflection coating on the front surface increases this difference.

\subsubsection{Comparison to the Ergodic Limit}

A $200 \mathrm{~nm}$ thick silicon layer has only one TM photonic mode and one TE mode; greater enhancements than shown in Fig. 5.5 are possible for thicker layers, which can reduce the relative importance of the comparatively lossy SPP mode. Figure 5.6 shows the absorption enhancement with respect to a single pass at normal incidence of an $800 \mathrm{~nm}$-thick silicon layer on silver as a function of dipole position for wavelengths near the absorption edge at $\lambda \approx 1100 \mathrm{~nm}$. In this case, the silicon layer is assumed to be covered with a perfect broadband antireflection coating, which eliminates all reflections at the front surface of the silicon (until the angle of incidence for light incident from within the silicon exceeds the critical angle). Recent coating layers of gradually varying index approach this idealized condition $[110,111]$ which we therefore take as a realistic limit. Furthermore, to explore the limiting enhancement possible with ideal dipoles, we assume $\Lambda=1$, independent of wavelength.

At any given wavelength, the absorption enhancement oscillates with dipole position, reflecting the changes in mode coupling that depend on the dipole position and 


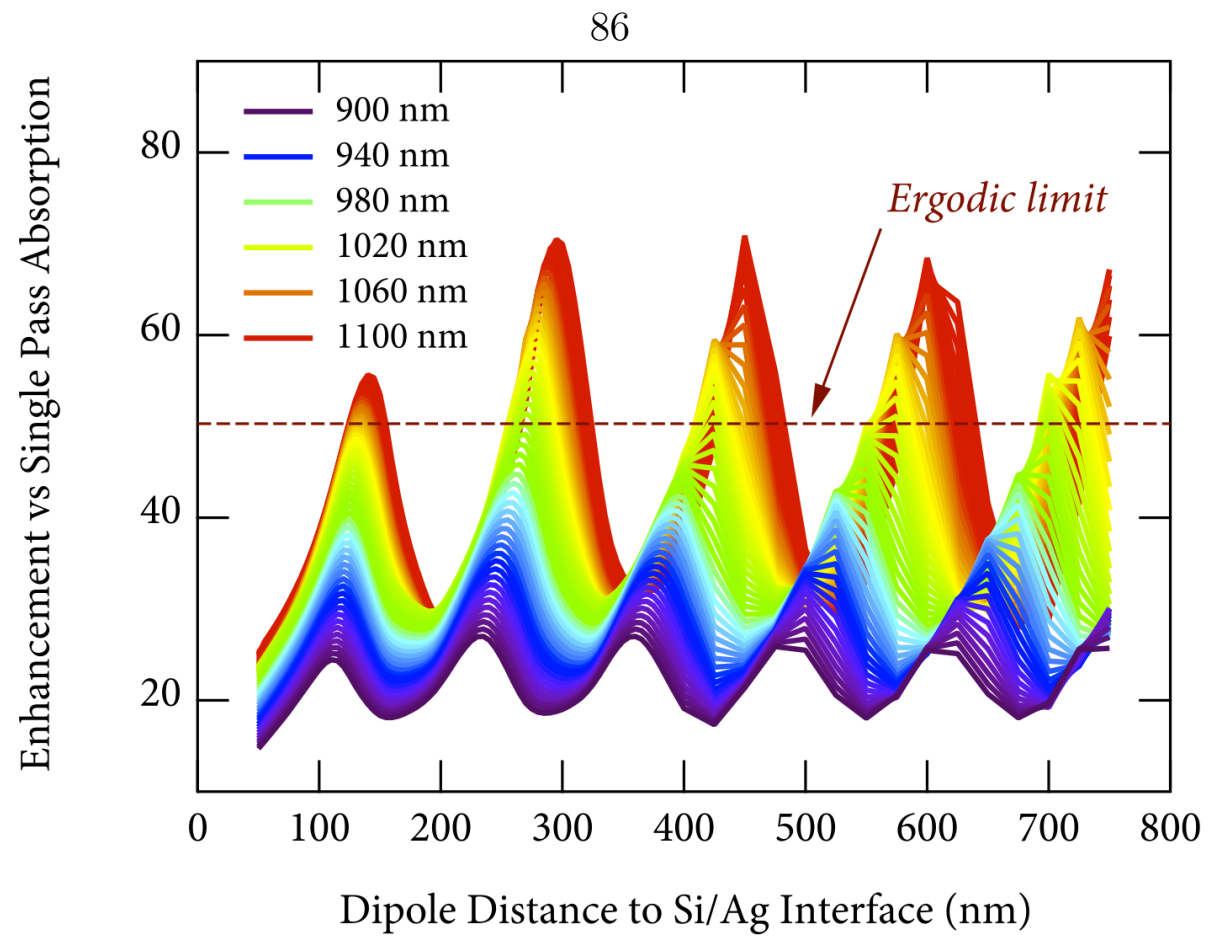

Figure 5.6. Absorption enhancement of light near the band edge caused by dipoles parallel to the surface of an $800 \mathrm{~nm}$ Si layer on Ag. The silicon is covered with a perfect antireflection coating, $\Lambda=$ 1 , and $L=1 \mu \mathrm{m}$. The dashed line shows the ergodic limit for $\lambda=1100 \mathrm{~nm}$ in a thick silicon layer. Note that a higher density of points has been computed for $d_{-}<400 \mathrm{~nm}$.

orientation (which here is assumed parallel to the interfaces). We might expect that enhancement would be greatest for dipole positions that couple most strongly to the TE modes that minimize the absorption in the metal. To investigate, we evaluate the coupling fractions into the various modes for dipoles at $200 \mathrm{~nm}$ from the $\mathrm{Ag}$, where enhancement at $1100 \mathrm{~nm}$ is minimum, and at $300 \mathrm{~nm}$ from the $\mathrm{Ag}$ interface, near the global maximum. The modes of this structure at $\lambda=1100 \mathrm{~nm}$, for which the fraction of power absorbed in a single pass at normal incidence is $\alpha d=8.4 \times 10^{-4}$, are shown in Fig. 5.7, along with the fraction absorbed in the silver and the silicon for each mode. The silicon fraction ranges from $0.35 \%$ for the SPP mode, to $56.1 \%$ for the fundamental TE mode. In addition, the coupling fractions to each mode are shown at the right of each figure for two positions of the dipoles, $d_{-}=200 \mathrm{~nm}$ (red), corresponding to an approximate minimum in enhancement, as seen in Fig. 5.6, and 
$d_{-}=300 \mathrm{~nm}$ (green), corresponding to a maximum.

Several remarks are in order. First, the coupling fractions confirm an intuitive interpretation of mode coupling: it scales with the magnitude squared of the field component parallel to the dipole, at the position of the dipole. Second, parallel dipoles this far from the metal interface couple much more strongly to TE modes than to TM modes, and coupling to the SPP mode is negligible. Third, the most significant coupling difference between these two positions is the fraction coupled to the escape cone at each scattering event. At the enhancement minimum, $11.2 \%$ of the scattered light couples to the escape cone, whereas at $d_{-}=300 \mathrm{~nm}$ only $0.4 \%$ escapes. We note again that this behavior describes dipoles oriented parallel to the layers; perpendicular dipoles might show less modulation. Successive peaks in the enhancement at $1100 \mathrm{~nm}$ are separated by about $150 \mathrm{~nm}$, which is half the wavelength in the silicon, $\lambda / n^{\prime}=310 \mathrm{~nm}$. At positions where emission from the dipole towards the silver reflects and returns to the dipole out of phase with the forward emission, near perfect destructive interference produces a minimum in emission, thanks to the high reflectivity of the $\mathrm{Si} / \mathrm{Ag}$ interface. Note that with the perfect antireflection coating, there is no reflection at the upper silicon surface. Fourth, dispersion shifts the position of the maxima increasingly with $d_{-}$, so smaller values of $d_{-}$lead to large enhancements over a broader range of wavelengths. However, coupling to the SPP mode leads to smaller enhancement at $d_{-}=135 \mathrm{~nm}$ than at $d_{-}=290 \mathrm{~nm}$ so that the greatest integrated enhancement in this structure requires $d_{-} \approx 260 \mathrm{~nm}$.

The greatest enhancement at the band edge for this structure is about 70 (Fig. 5.6). This is considerably greater than the $4 n^{2}=50.3$ enhancement of the ergodic limit, which assumes that absorption is negligible. Actually, $4 n^{2}$ is the limit for bulk layers; as Stuart and Hall have shown, it is somewhat reduced in thin layers [98]. Over a range of wavelengths near the band edge, ideal dipole scattering with properly positioned strong scatterers exceeds the ergodic limit by a wide margin. 

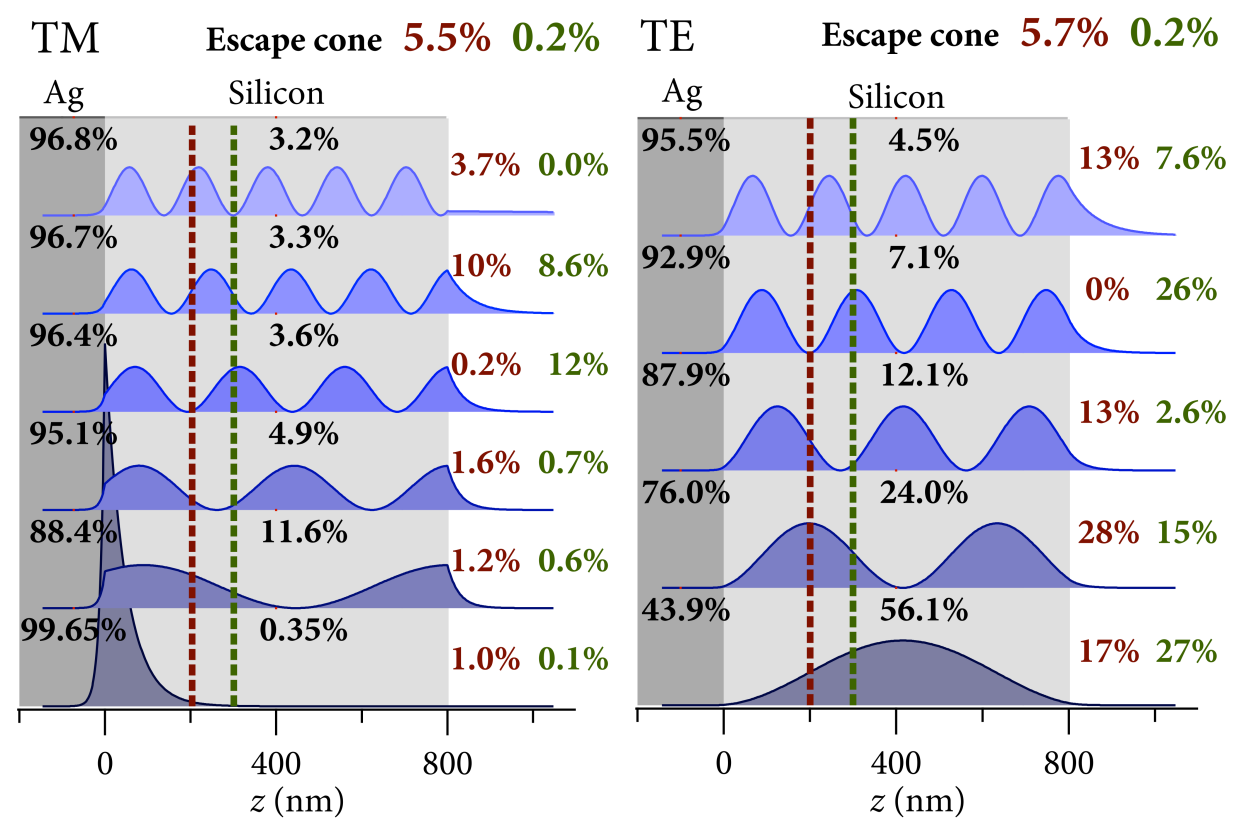

Figure 5.7. TM (left) and TE (right) modes of an $800 \mathrm{~nm}$ Si layer on $\mathrm{Ag}$ at $\lambda=1100 \mathrm{~nm}$. The shaded curves show the magnitude squared of the parallel component of electric field, and have equal area. The black numbers in each layer show for each guided mode the fraction absorbed in the layer (e.g., for the SPP mode at the lower left, $0.35 \%$ is absorbed in the silicon and $99.65 \%$ is absorbed in the silver). The fraction of light coupled into each mode for a parallel dipole positions $200 \mathrm{~nm}(300 \mathrm{~nm})$ from the $\mathrm{Ag}$ layer is shown in red (green) at the right of each figure; the fraction coupled into the escape cone is shown at the top. 


\subsubsection{Comparing Semiconductors}

While large absorption enhancements near the band edge help improve a cell's efficiency, of greater significance is the total absorption integrated from the band edge to the UV limit of the solar spectrum, which we take to be $\lambda_{\mathrm{UV}}=340 \mathrm{~nm}(3.65 \mathrm{eV})$. The spectrally integrated absorption efficiency (IAE) for a given spectral range is then the ratio of the number of photons absorbed in the semiconductor to the total number incident, per unit area.

For short wavelengths, absorption is strong enough to make enhancement mechanisms (texturing or dipole scattering) unnecessary, since most photons are absorbed before arriving at the metal interface. It is illustrative, therefore, to show the limiting IAE for the spectral regions both near the band edge and up to $\lambda_{\mathrm{UV}}$. Figure 5.8 shows these efficiencies for several semiconductor layer thicknesses in two weakly absorbing ( $\mathrm{Si}$ and $\mathrm{Ge}$ ) and two strongly absorbing (a-Si:H and GaAs) semiconductors. In all cases the upper surface is perfectly antireflection coated and the metallic substrate layer is silver. For $\mathrm{Si}$ and $\mathrm{Ge}$, the dipole is at $d_{-}=200 \mathrm{~nm}$ from the metal interface. For the thinner layers of a-Si:H and GaAs, $d_{-}$is the smaller of $d / 2$ and $100 \mathrm{~nm}$, where $d$ is the semiconductor layer thickness, to keep the dipoles from approaching the front surface.

Dipole scattering significantly enhances absorption in both weak and strong absorbers. Although the proportional increase in integrated IAE is greater for the weak absorbers (13.9 for Si and 6.8 for Ge vs. 4.4 for a-Si:H and 5.0 for GaAs at the smallest thickness considered for each material), the absolute increase is greater for the strong absorbers. A $100 \mathrm{~nm}$ a-Si:H layer with ideal scatterers spaced $500 \mathrm{~nm}$ apart absorbs $87 \%$ of the incident photons in the range $340 \mathrm{~nm} \leq \lambda \leq 840 \mathrm{~nm}$. Without the scatterers (i.e., in a structure with plane parallel interfaces), it would take a layer 7 times thicker to achieve the same absorption. 

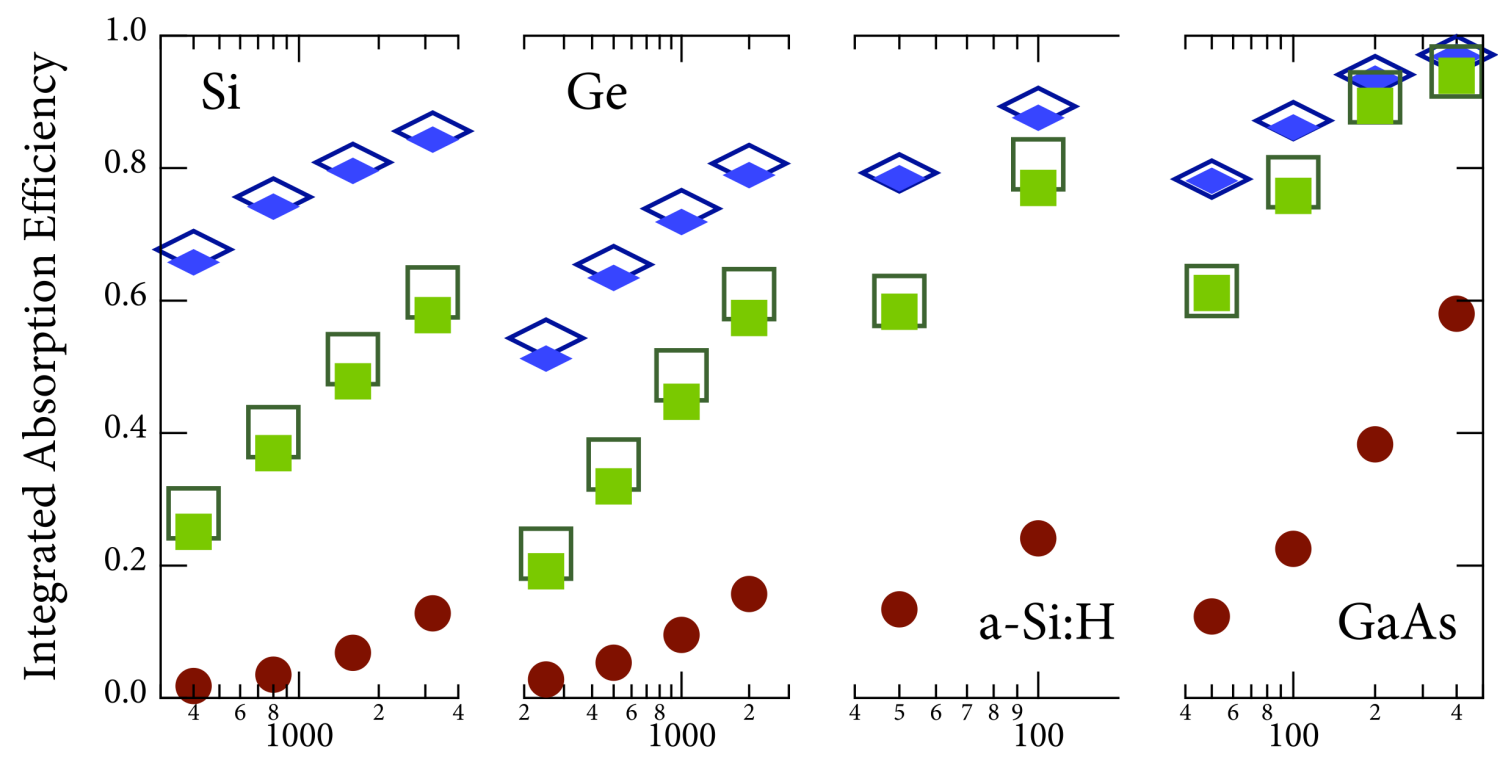

Semiconductor Thickness (nm)

Figure 5.8. Limiting integrated absorption efficiency (IAE) for thin layers of silicon, germanium, amorphous silicon, and gallium arsenide on optically thick Ag layers illuminated with the AM1.5G spectrum. The squares show the IAE for the spectral range near the band edge $\left(\frac{3}{4} \lambda_{g} \leq \lambda \leq \lambda_{g}\right)$, whereas the circles show the IAE for the same spectral range in the absence of dipole scatterers. The diamonds show IAE for the full spectrum $\left(\lambda_{\mathrm{UV}} \leq \lambda \leq \lambda_{g}\right)$. The semiconductor layers are covered with a perfect antireflection coating. The dipoles are separated by $500 \mathrm{~nm}$ for the filled symbols and by $1000 \mathrm{~nm}$ for the open symbols. 


\subsection{Assumptions of the Model}

We aim here to understand the theoretical limit to absorption enhancement in a thin metal-backed layer caused by dipole scatterers embedded in the absorbing layer. Accordingly, we have made a number of idealizations, some of which may be difficult to achieve experimentally. Specifically, we have assumed that:

1. the dipoles are strong enough and spaced closely enough such that they scatter all incident light $(\Lambda(\lambda)=1)$;

2. the dipoles do not significantly perturb the guided modes of the multilayer structure;

3. the multiple scattering at a given wavelength may be treated incoherently;

4. the dipoles are lossless scatterers $(\hat{g}=0)$;

5. losses in the optically thick metal layer on the substrate are described by the (bulk, local) complex dielectric function; and

6. the upper surface of the absorber is covered by a perfect antireflection coating.

Given these assumptions, we find that for both indirect-gap materials, such as silicon and germanium, and direct or quasi-direct-gap materials, such as gallium arsenide and amorphous silicon, lossless dipole scatterers that interact strongly with above-gap radiation can very significantly enhance absorption in the semiconductor, even when Joule losses in the metal substrate layer are accounted for. But, are the assumptions reasonable?

Approaching the strongly coupled limit $(\Lambda=1)$ may be possible, at least at selected wavelengths. Using Mie theory [71] we find that silver spheres with $50 \mathrm{~nm}$ radius embedded in silicon have a scattering cross section $Q_{\text {sca }}=6.6$ times their geometric cross section at $\lambda=880 \mathrm{~nm}$, and an average $Q_{\text {sca }}$ greater than 5 over the range 800-1100 nm. Silver ridges $100 \mathrm{~nm}$ wide and spaced about $500 \mathrm{~nm}$ apart may scatter a fraction approaching unity of incident plane waves, at least for wavelengths 
in the infrared. As shown in Fig. 5.8, while scatterers spaced $1 \mu \mathrm{m}$ apart are more efficient, the difference compared to $500 \mathrm{~nm}$ spacing is modest. It may be difficult to realize appropriately spaced embedded scatterers without introducing excessive surface recombination, and without strongly perturbing the multilayer modes we have assumed. Metallic ridges $50 \mathrm{~nm}$ tall in a layer $100 \mathrm{~nm}$ thick will produce significant Fabry-Perot resonances that alter the radiative density of states. However, this effect would be sharply reduced for thicker absorbing layers, such as the $800 \mathrm{~nm}$ layers of Fig. 5.7. Thin metallic islands on dielectric pedestals penetrating into the semiconductor might alter the pure multilayer modes less than ridges, yet maintain their large scattering efficiency.

Our treatment of the dipoles as independent scatterers is supported by absorption enhancement caused by silver islands on thin silicon layers [100]. As regards the fourth assumption, we have focused on metallic scatterers because strong plasmon resonances enable these structures to have very large scattering cross sections, as indicated above. Furthermore, scattering dominates absorption at these sizes. For the same 800-1100 $\mathrm{nm}$ range that has $\bar{Q}_{\mathrm{sca}}=5.1$, the dimensionless total extinction cross section is $Q_{\text {ext }}=5.76$, indicating that 8 times more power is scattered than absorbed in the metal. We have calculated principally for silver, which has the least loss of any metal across the relevant spectral range. Less expensive and more abundant metals, such as copper, may be worth exploring, particularly for infrared application where losses are quite similar to silver. Furthermore, we have assumed a single value for the coupling coefficient $\Lambda$ in producing Fig. 5.8, although scattering efficiency is apt to decrease with wavelength into the visible. We have also taken $\Lambda$ independent of the position of the dipoles with respect to the metal/absorber interface. This assumption is bolstered by looking at the integrated decay rate, $\hat{\gamma}$, for perpendicular and parallel dipoles as a function of $d_{-}$. For $d_{-}$greater than about $80 \mathrm{~nm}, \hat{\gamma}$ is within $\approx 25 \%$ of unity, independent of the dipole position. So, while the fraction coupling to each mode changes with $d_{-}$, the total coupling is fairly constant. Furthermore, variations in $\hat{\gamma}$ are significant only in comparison to alternative energy pathways, which we take as negligible. We will explore the influence of absorption in the dipoles $(\hat{g}>0)$ and 
declining scattering with wavelength in a subsequent publication.

The fifth assumption is entirely reasonable, as we find that the scatterers should be placed much more than $10 \mathrm{~nm}$ from the metal plane, far enough that surface effects are entirely negligible. Finally, efficient absorption in any geometry requires minimizing reflection losses, and achieving optimal efficiency demands an ideal antireflection coating. Whether broadband AR coatings will be economically feasible is an interesting but separate question. Regardless, the dipole-scattering mechanism enhances absorption whether front-surface reflections are suppressed or not.

\subsection{Conclusion}

We have elaborated a quasi-analytic theory of absorption in multilayer structures with independent embedded dipole scatterers. Unlike full-field simulations using FDTD, the method uses experimental dielectric functions and runs quite quickly, allowing us to explore parameter space more efficiently. In this paper, we have focused on structures with optically thick metal back planes, which support both plasmonic and photonic guided modes. We find that while absorption losses at optical frequencies are greatest in SPP modes, they do not spoil the very significant absorption enhancement in a broad variety of structures. When these ideal dipole scatterers couple strongly enough to scatter essentially all incident light, yet are spaced roughly $500 \mathrm{~nm}$ part, they enable absorption of greater than $85 \%$ of above-gap solar radiation in AR-coated thin absorber layers.

\subsection{Appendix}

The fraction of the power in a guided mode that is absorbed in each lossy layer may be computed by considering the power flux into each layer through the plane at $x=0$ and between layers through the interfaces that separate them, as illustrated in Fig. 5.1. The average power per unit area carried by the monochromatic electromagnetic field is given by the average Poynting vector, $\mathbf{S}=\frac{c}{8 \pi} \Re\left(\mathbf{E} \times \mathbf{H}^{*}\right)$. 
Consider a layer bounded below at $z=0$ and above by $z=d$. A TM-polarized mode has the form

$$
\begin{aligned}
& \mathbf{E}(\mathbf{r}, t)=\left[E_{+}\left(\frac{-u_{z} \hat{\mathbf{x}}+u_{x} \hat{\mathbf{z}}}{n}\right) e^{i k_{z} z}+E_{-}\left(\frac{u_{z} \hat{\mathbf{x}}+u_{x} \hat{\mathbf{z}}}{n}\right) e^{-i k_{z} z}\right] e^{i\left(k_{x} x-\omega t\right)} \\
& \mathbf{H}(\mathbf{r}, t)=-\left(E_{+} e^{i k_{z} z}+E_{-} e^{-i k_{z} z}\right) n e^{i\left(k_{x} x-\omega t\right)} \hat{\mathbf{y}}
\end{aligned}
$$

where the amplitudes $E_{ \pm}$are those at the back of the layer at $z=0$, and I have taken $\mu=1$. The power flux into the layer along the $x=0$ plane, per unit length in the $y$ direction, is then

$$
\begin{aligned}
\Phi_{x}^{\mathrm{TM}}= & \int_{0}^{d} S_{x} d z=\frac{c}{16 \pi} u_{x}^{\prime \prime} \Re\left[\frac { n ^ { * } u _ { x } } { n } \left\{\frac{\left|E_{+}\right|^{2}\left(1-e^{-2 u_{z}^{\prime \prime} \phi}\right)}{u_{z}^{\prime \prime}}+\frac{\left|E_{-}\right|^{2}\left(e^{2 u_{z}^{\prime \prime} \phi}-1\right)}{u_{z}^{\prime \prime}}\right.\right. \\
& \left.\left.+\frac{2 \Im\left(E_{+} E_{-}^{*}\right) \sin \left(2 u_{z}^{\prime} \phi\right)}{u_{z}^{\prime}}+\frac{2 \Re\left(E_{+} E_{-}^{*}\right)\left[\cos \left(2 u_{z}^{\prime} \phi\right)-1\right]}{u_{z}^{\prime}}\right\}\right]
\end{aligned}
$$

where $\phi=\widetilde{\omega} d=2 \pi d / \lambda, \lambda$ is the vacuum wavelength, the field amplitudes $E_{ \pm}$ are those at $z=0$, and the real and imaginary parts of a complex number $\zeta$ are represented by $\zeta^{\prime}$ and $\zeta^{\prime \prime}$, respectively. Similarly, the flux per unit length in the $y$ direction through a plane perpendicular to the $z$ - axis for $x \geq 0$ are

$\Phi_{z}^{\mathrm{TM}}(z)=\int_{0}^{\infty} S_{z}(z) d x=\Re\left\{\frac{c n^{*} u_{z}}{16 \pi n}\left[\left|E_{+}\right|^{2} e^{-2 k_{z}^{\prime \prime} z}-\left|E_{-}\right|^{2} e^{2 k_{z}^{\prime \prime} z}+2 i \Im\left(E_{+} E_{-}^{*} e^{-2 i k_{z}^{\prime} z}\right)\right]\right\}$

We note that in the case of the incident medium (for which $d \rightarrow \infty$ ), the terms involving $E_{-}$vanish in a bound mode; in the substrate $E_{+}=0$.

For a TE mode, the analogous expressions are 


$$
\begin{aligned}
\mathbf{E}(\mathbf{r}, t)= & \left(E_{+} e^{i k_{z} z}+E_{-} e^{-i k_{z} z}\right) e^{i\left(k_{x} x-\omega t\right)} \hat{\mathbf{y}} \\
\mathbf{H}(\mathbf{r}, t)= & {\left[E_{+}\left(-u_{z} \hat{\mathbf{x}}+u_{x} \hat{\mathbf{z}}\right) e^{i k_{z} z}+E_{-}\left(u_{z} \hat{\mathbf{x}}+u_{x} \hat{\mathbf{z}}\right) e^{-i k_{z} z}\right] e^{i\left(k_{x} x-\omega t\right)} } \\
\Phi_{x}^{\mathrm{TE}}= & \frac{c u_{x}^{\prime} u_{x}^{\prime \prime}}{16 \pi}\left[\frac{\left|E_{+}\right|^{2}\left(1-e^{-2 u_{z}^{\prime \prime} \phi}\right)+\left|E_{-}\right|^{2}\left(e^{2 u_{z}^{\prime \prime} \phi}-1\right)}{u_{z}^{\prime \prime}}\right. \\
& \left.+\frac{2}{u_{z}^{\prime}}\left(\Re\left[E_{+} E_{-}^{*}\right] \sin \left(2 u_{z}^{\prime} \phi\right)-\Im\left[E_{+} E_{-}^{*}\right]\left[1-\cos \left(2 u_{z}^{\prime} \phi\right)\right]\right)\right] \\
\Phi_{z}^{\mathrm{TE}}(z)= & \frac{c}{16 \pi}\left[u_{z}^{\prime}\left(\left|E_{+}\right|^{2} e^{-2 k_{z}^{\prime \prime} z}-\left|E_{-}\right|^{2} e^{2 k_{z}^{\prime \prime} z}\right)+2 u_{z}^{\prime \prime} \Im\left(E_{+}^{*} E_{-} e^{-2 i k_{z}^{\prime} z}\right)\right]
\end{aligned}
$$




\section{Chapter 6}

\section{Improved Red Response in a-Si:H Solar Cells with Soft-Imprinted Back Reflectors}

\subsection{Introduction}

Having explored the possibilities for coupling to modes from different back contact nanostructures, as well as the relative benefits to each mode, we now turn our attention to experimental devices. We chose to work with a-Si:H as it is can benefit strongly from improved light trapping: indeed, all commercial a-Si:H solar cells employ light trapping surfaces to increase path length while preserving small absorber volumes. The appropriate reference for our light trapping designs is thus not photocurrent enhancement over a non-textured cell, but photocurrent enhancement over an a-Si:H cell containing state-of-the-art, non-plasmonic light trapping textures. While applied here to a-Si:H, the nanopatterning technique and design principles are general and applicable to other materials systems. This chapter details the fabrication of large-scale, nanopatterned a-Si:H solar cells and demonstrates ultrathin film plasmonic solar cells with enhanced photocurrent beyond randomly textured reference cells.

\subsection{Advantages of Light Trapping for a-Si:H}

Thin film solar cells made from a-Si:H are attractive candidates for large-scale photovoltaic applications because Si is highly abundant and can be deposited on flexible substrates using processes that are compatible with roll-to-roll processing [11]. Since minority carrier diffusion lengths are very short in a-Si:H, the cells are often made 
with a p-i-n or n-i-p structure where the intrinsic absorbing layer is hundreds of nanometers thick and carrier transport is dominated by drift. The difference between $\mathrm{p}-\mathrm{i}-\mathrm{n}$ and $\mathrm{n}-\mathrm{i}-\mathrm{p}$ cells is the order of the layer deposition: $\mathrm{p}-\mathrm{i}-\mathrm{n}$ cells are built in a superstrate configuration starting from the transparent conducting oxide front contact, and n-i-p cells are built starting from the metal back contact. The order of deposition determines which surface includes the initial light trapping structures: p-i-n cells typically incorporate texture from the transparent conducting oxide superstrate, and n-i-p devices from the metallic substrate.

Ultrathin film cells, where the thickness of the absorber layer is significantly reduced, offer further cost and performance advantages relative to their thin film counterparts. While ultrathin absorbing layers clearly reduce the cost of feedstock materials, the more substantial cost advantage is throughput: plasma enhanced chemical vapor deposition processes are typically the rate-limiting step in thin film Si solar cell production, and a reduction in necessary thickness dramatically improves the speed of fabrication. Furthermore, an ultrathin film absorbing layer exhibits decreased bulk recombination, which can lead to a higher open circuit voltage $\left(\mathrm{V}_{\text {oc }}\right)$, since $\mathrm{V}_{\text {oc }}$ increases with decreasing dark current $I_{d a r k}$ as $V_{o c}=\frac{k T}{q} \ln \frac{I p h o t o}{\text { Idark }+1}$ [13]. A final important benefit of this ultrathin film design for a-Si:H is the reduction of the well-known Staebler-Wronski degradation effect that has limited the long-term performance of a-Si:H photovoltaics so far [14]. Ultrathin n-i-p devices, with thicknesses such as we discuss here, possess high internal electric fields, which are known to exhibit no or only minimal light-induced degradation $[11,15,112]$.

However, the use of thin absorbing layers reduces the short-circuit current density $\mathrm{J}_{\mathrm{sc}}$ due to the decreased optical path length in the semiconductor. Strategies for increasing $\mathrm{J}_{\mathrm{sc}}$ generally involve the incorporation of surface texturing to scatter incident light into off-normal angles. In thick, wafer-based photovoltaic cells, such surface texturing can lead to a maximum intensity enhancement of $4 n^{2}$ at wavelengths near the band edge, where $n$ is the index of refraction of the semiconductor [16]. For thin and ultrathin film cells where the total device thickness may be less than a wavelength, light trapping is frequently accomplished through the use of randomly 
roughened layers, either on the front or rear of the cell $[113,114,115,116,117]$. Surface texturing has also been achieved by incorporating roughness in electrically passive materials, such as plastic substrates [118] and glass superstrates [119]. There are non-optical device benefits to optimization of the improved light management as well: reducing the surface topography relative to a randomly structured rear contact improves semiconductor film conformity and thereby electronic quality [120].

Recently, it has been proposed that the incorporation of plasmonic metal nanostructures in thin film solar cells could lead to strong light trapping because strong light-matter interaction in plasmonic nanostructures enables large scattering cross sections [25, 121]. Many of these plasmonic photovoltaic designs incorporate metal nanoparticles on the front surface of the cell. This can lead to preferential scattering of the incident light into the semiconductor over an increased angular range, thereby enhancing the optical path length $[46,47,48,50,57,60,69,101]$. While strong enhancements of photocurrent have been reported in such solar cells for near-bandedge light, these are often offset by a reduced photocurrent in the blue part of the solar spectrum due to destructive Fano interference $[48,101]$. An alternative strategy is to build the scattering nanostructures directly into the back contact of the device. While many semiconductor materials cannot be grown with high quality over rough surfaces, this is not an issue for thin film a-Si:H. In this geometry, the incident blue light is directly absorbed and does not interact with the back contact scatterers, while the red light that is poorly absorbed in a single pass through the cell is strongly scattered $[20,32,67]$. In our design, these back scatterers are designed to couple incident light into guided modes supported by the cell with high intensity in the absorbing semiconductor layer, dramatically reducing the thickness requirements by redirecting the absorption path into the plane of the solar cell. As opposed to cells with purely grating-based reflectors $[26,27,28,122]$ or nanostructure designs to couple specifically to surface plasmon polariton modes [67], the design presented here takes advantage of the high scattering cross sections of plasmonic nanostructures to couple to photonic waveguide modes. 


\subsection{Nanofabrication: Substrate Conformal Imprint Lithography}

A significant challenge to the incorporation of plasmonic nanostructures in photovoltaics is fabrication: the feature sizes are typically tens to hundreds of nanometers, while photovoltaic cell dimensions may be in the $\mathrm{m}^{2}$ range. Techniques for large area metal nanostructure formation include island annealing, which produces irregular shapes and spacings [46, 57], colloidal particles [47, 60], which control the shape but not the spacing, and deposition through alumina templates, which can provide some control over the patterns, but with imperfect ordering $[48,50]$. Smaller test devices may use electron-beam lithography or focused ion beam patterning, which allow for complete control but are too expensive for use in practical devices. In this work, we have fabricated $\mathrm{cm}^{2}$-scale a-Si:H solar cells using Substrate Conformal Imprint Lithography (SCIL) [123] to incorporate plasmonic nanostructures in the $\mathrm{Ag} / \mathrm{ZnO}: \mathrm{Al}$ back contact of the cell.

We use soft poly-di-methyl-siloxane (PDMS) stamps molded from a master pattern to emboss nanopatterns into a silica sol-gel resist. While the initial master fabrication may be expensive, both the master pattern and the PDMS stamps are reusable thousands of times, which substantially reduces the cost of implementation. This technique offers the capability to form large-area nanopatterns with precise control over both the dimensions and the spacing of the plasmonic scattering structures, and is amenable to roll-to-roll processing [124].

An initial master pattern is first made on a Si wafer. The master can be fabricated by any standard method for patterning, such as electron beam lithography, focused ion beam milling, or laser interference lithography. The Si master is passivated to avoid reaction with PDMS with 1,1,2,2H-perfluorodecyl-tri-chlorosilane in a vacuum oven at $50{ }^{\circ} \mathrm{C}$ for 16 hours $[125,126]$. The chloro-silane group passivates the Si-H and $\mathrm{Si}-\mathrm{OH}$ bonds on the surface, while the fluorine-modified tails help to form an inert close packed layer[127]. The surface passivation layer prevents the PDMS from sticking too strongly to the Si wafer, so that a stamp can be peeled off gently without 


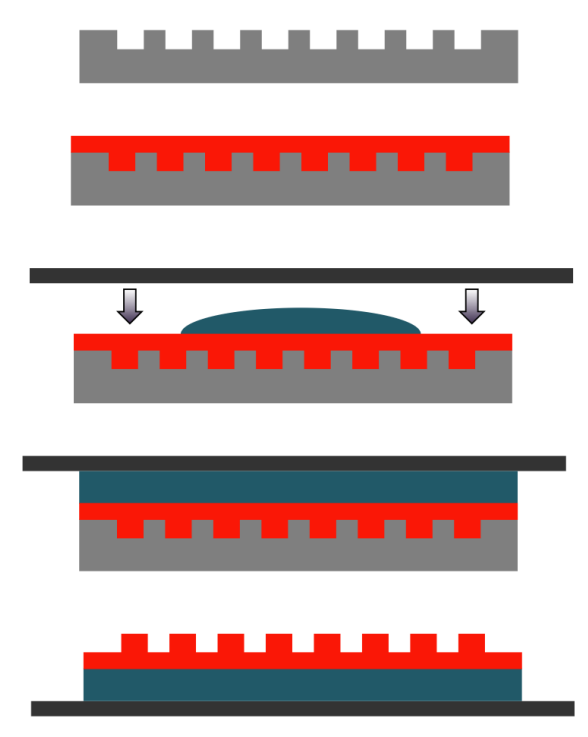

(a)

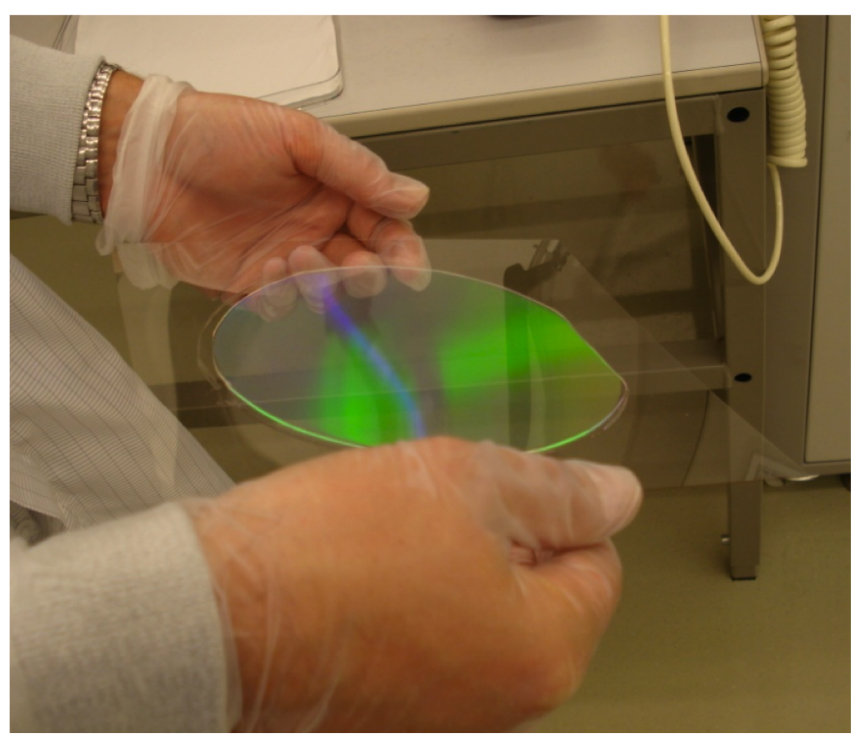

(b)

Figure 6.1. (a)Overview of Stamp Fabrication. 1. Patterned Si Wafer. 2. Cast high-modulus PDMS. 3. Cast low modulus PDMS and affix to AF45 glass backing. 4. Cure. 5. Remove stamp from wafer. (b) Photograph of SCIL stamp: Composite PDMS on AF45 glass backing sheet.

breaking the master.

The PDMS stamp used here is a composite stamp, consisting of two layers of PDMS with different Young's modulus and a glass backing sheet, and the process is illustrated in Fig. 6.1(a). PDMS stamps are advantageous over other nanoimprint lithography stamps (such as harder quartz stamps) due to their conformal contacting of the substrate and easy removal from the master. While the most common materials for soft imprint use standard PDMS formulations (Sylgard 184 from Dow Corning), this can lead to pattern collapse for dense arrays of nanostructures. A second common disadvantage of PDMS stamps is that particularly small features become rounded due to surface tension. To get around this problem, the SCIL method uses a bilayer stamp formulation, consisting of a higher modulus PDMS layer in contact with the nanopatterns, bonded to a lower modulus PDMS to provide flexibility in the backing layer [128]. The high modulus PDMS cannot be used alone as it is fragile, and the bulk 
may distort when the stamp has to conform around large contaminant particles on the substrate. The stamps are made by pouring liquid over the passivated Si master, then thermally curing the liquid to promote cross-linking and rubber formation. The PDMS stamp is then affixed to a flexible glass sheet, of $200 \mu \mathrm{m}$ glass. The glass has out-of-plane flexibility, which allows for conformation of the stamp over a substrate, and in-plane stiffness which minimizes pattern distortion. The glass particularly helps avoid pattern distortion during curing of the PDMS over the Si wafer. A photograph of the SCIL stamp is shown in 6.1(b).

The stamps can be imprinted into a resist by hand, gently rolling the stamp into the resist, but this method tends to include air pockets which cause defects in the replication. In this work we used the SCIL tooling to emboss a silica sol-gel resist over a $\mathrm{cm}^{2}$ area with minimal defects [129]. The process is shown in Fig. ??. The tooling consists of a vacuum plate that the stamp is affixed to, containing a series of grooves. The stamp is brought in close contact to the resist-coated substrate, and the grooves are sequentially overpressurized, pulling the stamp off of the plate and into the resist by capillary force. The stamp is left in place until the resist is cured, and then the grooves are sequentially evacuated to pull the stamp gently from the imprinted resist. The imprint process requires very low pressure, making it compatible with brittle substrates.

While the method can be used with other resist materials, we use a silica sol-gel resist on glass. The rubber stamp is an active component of the silica sol-gel curation. Remaining air in the features diffuses into the stamp, followed by diffusion of the solvents into the stamp. The resist hardens by forming a dense network of $\mathrm{Si}-\mathrm{O}-\mathrm{Si}$ bonds, while the reaction products (water and alcohols) diffuse into the stamp. After densification, this forms a patterned $\mathrm{SiO}_{2}$ layer. The liquid resist consists mainly of silica sol-gel precursors, with some organic components to prevent shrinkage and cracking after hardening. The replicated sol-gel imprint is 88 wt- $\%$ silicon oxide, non-absorbing, and stable in air up to $450^{\circ} \mathrm{C}$. 
103
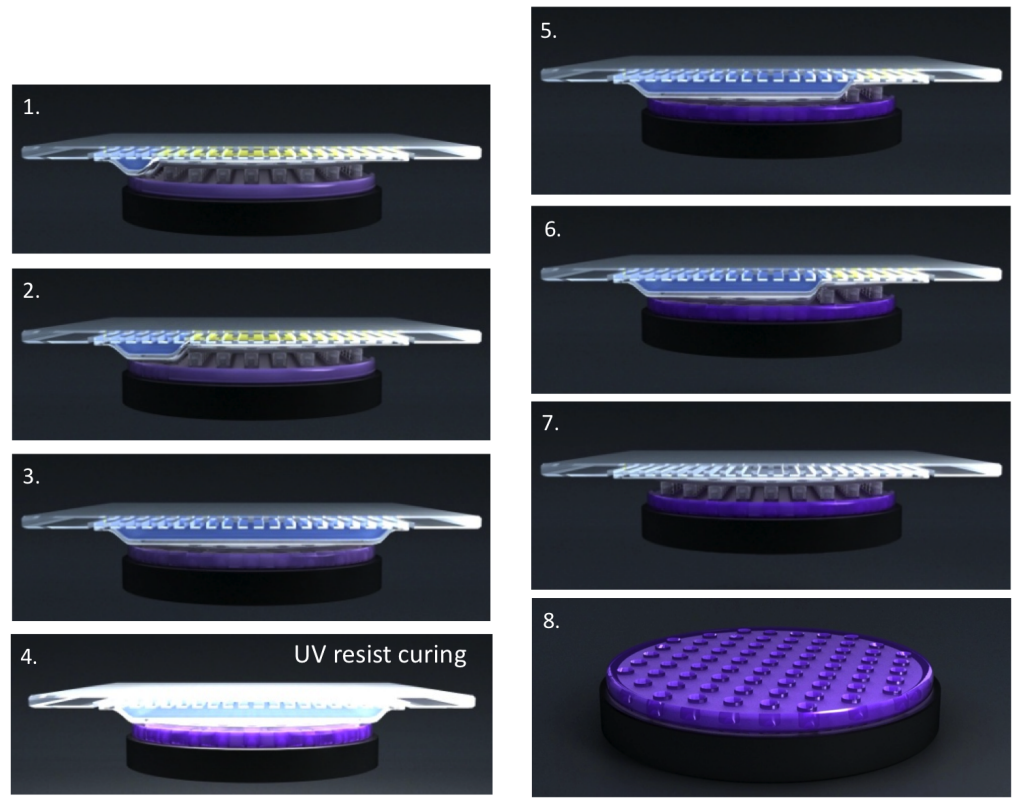

(a) Imprint

(b) Release

Figure 6.2. Overview of SCIL imprint process, showing low pressure imprint and release.

\subsection{Cell Fabrication}

For all of the experimental devices discussed here and in Chapter 8, we use a similar fabrication procedure. The stamp is imprinted into silica sol-gel resist on glass, and overcoated with $100 \mathrm{~nm}$ of $\mathrm{Ag}$ and $130 \mathrm{~nm}$ of $\mathrm{ZnO}$ :Al by sputtering. This forms a very standard back contact for a-Si:H device fabrication. The n-i-p a-Si:H layers are grown over the patterned $\mathrm{Ag} / \mathrm{ZnO}$ :Al layers using 13.56 MHz plasma enhanced chemical vapor deposition (PECVD), then $80 \mathrm{~nm}$ of ITO is sputtered through a shadow mask over the p-a-Si:H to form the top contact. The $80 \mathrm{~nm}$ thickness is chosen to act as an anti-reflection layer, with the reflection minimized at $\lambda=600$ $\mathrm{nm}$. This calculation was done for a cell with a flat top, and so may not be the ideal thickness for the devices fabricated here. As $80 \mathrm{~nm}$ is too thin to form a continuous layer, a Au contact grid is evaporated over the ITO to improve the series resistance. In all cases, the ITO is sputtered through a contact mask to form a $4 \mathrm{~mm} \mathrm{x} 4 \mathrm{~mm}$ cell, with an area of $0.16 \mathrm{~cm}^{2}$. The contact grid area is large $\left(0.03 \mathrm{~cm}^{2}\right)$, so the 


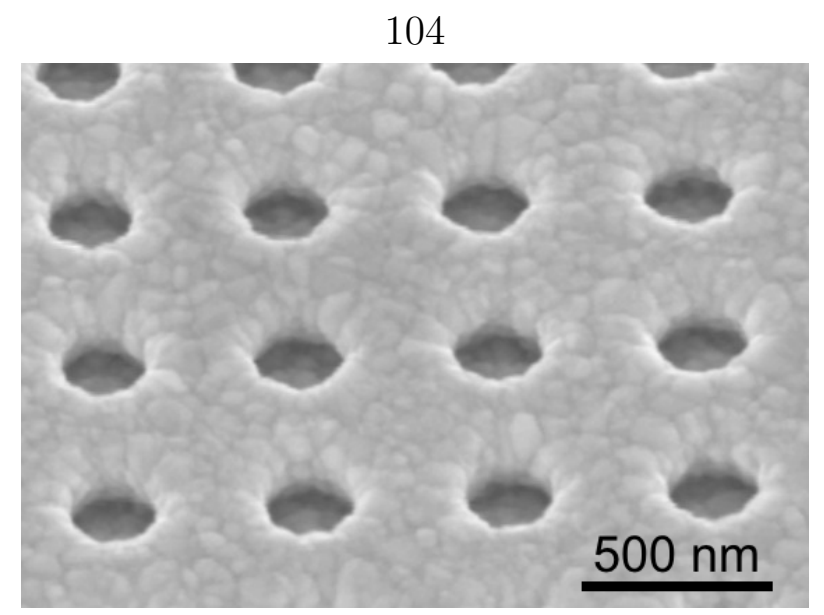

Figure 6.3. SEM image of a nanoimprinted pattern of holes after overcoating with Ag. The coated holes are $225 \mathrm{~nm}$ in diameter, 240 $\mathrm{nm}$ deep, and have a pitch of $513 \mathrm{~nm}$.

area used to calculate $\mathrm{J}_{\mathrm{sc}}$ is the total area less the area of the contact grid, 0.13 $\mathrm{cm}^{2}$. Further fabrication details will be provided in sections 6.5 and 6.6 as related to different experiments.

\subsection{Improving Red Response of a-Si:H Solar Cells with Hole Arrays}

Our first $\mathrm{Si}$ wafer master pattern was formed by laser interference lithography. The pattern consists of a hole array with $513 \mathrm{~nm}$ pitch, where each hole is $350 \mathrm{~nm}$ in diameter and $200 \mathrm{~nm}$ deep. The entire wafer contained the same nanopattern. A stamp was molded from this master, then transferred via the silica sol-gel resist into another Si wafer. After overcoating, the substrate contained Ag holes $225 \mathrm{~nm}$ in diameter, as illustrated in Fig. 6.3. At the same time, a reference sample was made by evaporating $\mathrm{Ag}$ and sputtering $\mathrm{ZnO} \mathrm{Al}$ over flat glass. The two substrates are then processed side by side to ensure identical a-Si:H deposition.

Figures 6.4(a) and 6.4(b) show cross sections of the completed solar cells made using FIB milling. The a-Si:H layers are $500 \mathrm{~nm}$ thick. This corresponds to approximately $45 \mathrm{~nm}$ of the doped layers, and $455 \mathrm{~nm}$ intrinsic regions. The $\mathrm{Ag}$ and $\mathrm{ZnO} \mathrm{Al}$ conformally coat the inside of the hole array, but the a-Si:H does not completely 


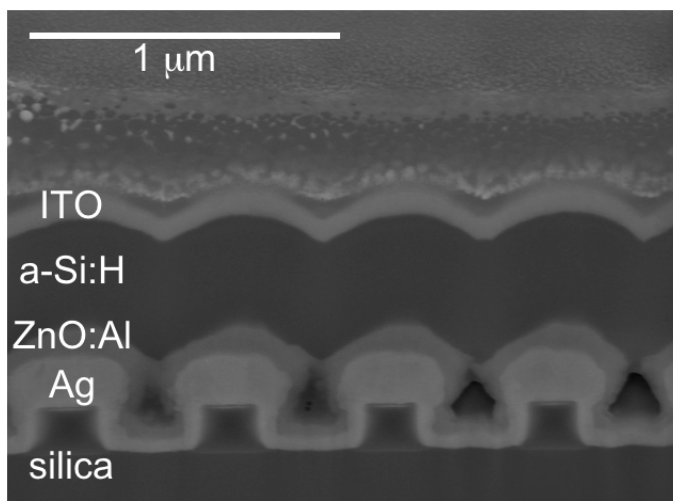

(a)

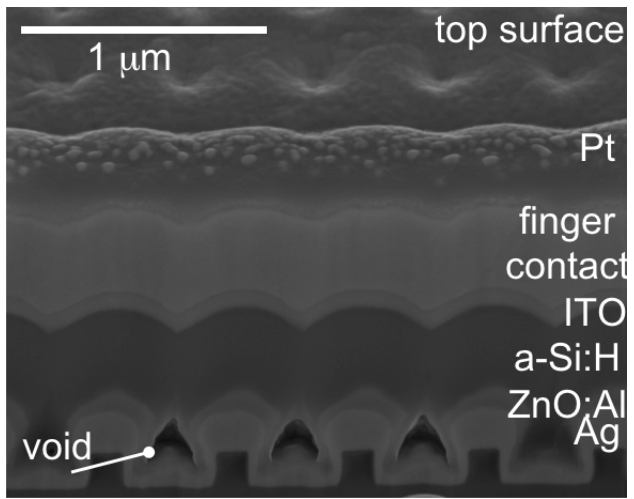

(b)

Figure 6.4. SEM cross section images of solar cell deposited over hole array.

coat the inside of the holes. The $\mathrm{Ag} / \mathrm{ZnO}$ :Al closes off the top, possibly creating a low-index vacuum scatterer inside the hole array, as is particularly clear in 6.4(b). These features are critical for accurate electromagnetic modeling of the hole array, and may also be detrimental for good contact performance. It is also evident from these cross sections that the top of the solar cell is nanostructured with slight dips on the positions of the holes in the underlying contact.

Figure 6.5 illustrates the current-voltage characteristics of the flat and patterned n-i-p a-Si:H cells for the best cell of each type. The J-V characteristics were measured with a solar simulator under one sun illumination (AM1.5G, $100 \mathrm{~mW} / \mathrm{cm}^{2}$ ). The patterned cell exhibits a $26 \%$ higher $\mathrm{J}_{\mathrm{sc}}$ than the flat cell, demonstrating an increased optical path length in the device. The $\mathrm{V}_{\text {oc }}$ shows a slight decrease, by $2 \%$. Combined, there is a significant increase in efficiency from $4.5 \%$ to $6.2 \%$ due to the patterned metal back contact. Several cells of each type were measured, with a variance in the absolute efficiency of 0.1 for the reference cell and 0.13 for the patterned cell. The variation in the fill factor is likely due to differences between the contacts, but it is interesting to note that the patterned cell does not suffer from a reduced series resistance or fill factor. The slight S-shape of the J-V curve in 6.5 is most likely due to a barrier between the p-a-Si:H and the ITO, and was corrected in later experiments.

To better understand the nature of the enhancement, we measured spectral re- 
106

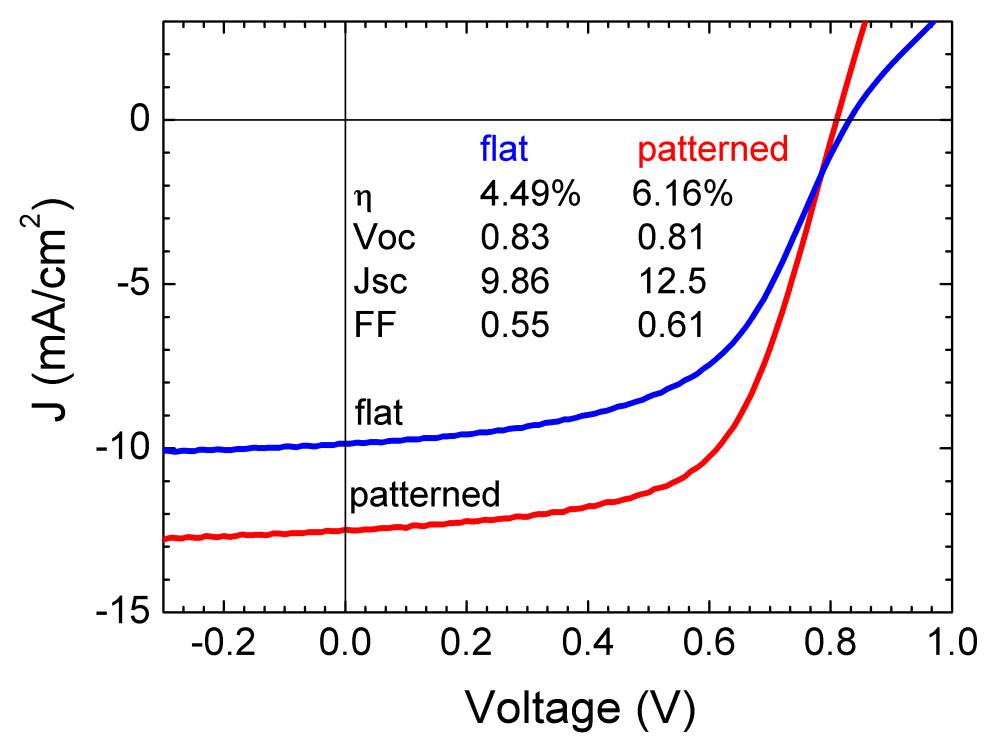

Figure 6.5. Best JV measurements of the flat reference and patterned n-i-p a-Si:H cells. Inset shows the cell characteristics for each device.

sponse curves for the two sets of devices. Figure 6.6 shows the spectral response measured under a reverse bias of -1 Volt, which facilitates comparison to optical simulation results by sweeping out all of the generated electrons. At wavelengths shorter than $550 \mathrm{~nm}$ there is little difference between the flat and patterned cells. This contrasts with designs for light trapping incorporating metal nanoparticles on top, which often see a decrease in efficiency in this part of the spectrum [69, 101]. At these wavelengths most of the light is absorbed in the $500 \mathrm{~nm}$ thick a-Si:H layer before interacting with the scattering layer. At wavelengths longer than $600 \mathrm{~nm}$ there is a significant difference in photocurrent between the two cells. Integrating over the 600 - $800 \mathrm{~nm}$ region finds a $51 \%$ increase in photocurrent.

While these experimental results are promising, the presence of voids in the hole arrays (Fig. 6.4), combined with the modeling results discussed in Chapter 4, motivated a change to pillar arrays rather than continuing work on hole arrays. The results are also incomplete, since they demonstrate only improvement over a flat back reflector rather than photocurrent enhancement over a randomly textured solar cell. They do, however, validate the concept that patterns built into the back contact of an a-Si:H solar cell improves the red response of the photocurrent without degrading 


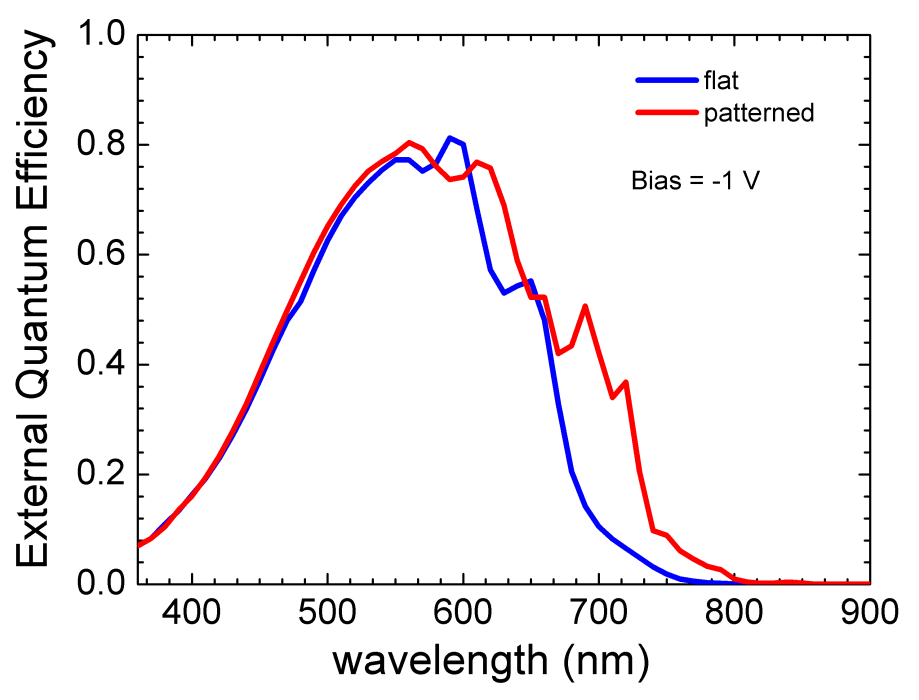

Figure 6.6. Measured external quantum efficiency for flat reference cells and cells made over hole array pattern.

the blue response.

\subsection{Fabrication of Pillar Array Solar Cells}

Besides the switch to pillar arrays, we also sought to design an experiment where multiple patterns could be tested on a single substrate. A new master was fabricated over a $10 \mathrm{~cm} \mathrm{x} 4 \mathrm{~cm}$ footprint, consisting of 16 rows of 6 cells each. Each pattern area is $6 \mathrm{~mm} \times 6 \mathrm{~mm}$, to aid in alignment of the $4 \mathrm{~mm} \times 4 \mathrm{~mm}$ ITO shadow masks after a-Si:H layer deposition. The master pattern was made by electron beam lithography. Photographs of the cell layout are shown in Fig. 6.7. Figure 6.7(a) shows the solar cells head-on, and it is visually clear that some of the patterns are more strongly absorbing than others. We chose 11 different nanopatterns, and tiled them with rotation and repetition over the substrate to ensure that all device measurements are repeatable and due to light trapping rather than deposition variation over the scale of the substrate. Each nanostructure is a square pillar with a specified diameter. Eight of the patterns are square periodic arrays, two are staggered arrays, and two are flat reference cells. Figure 6.7(b) shows the same substrate under an angle with 


\section{8}

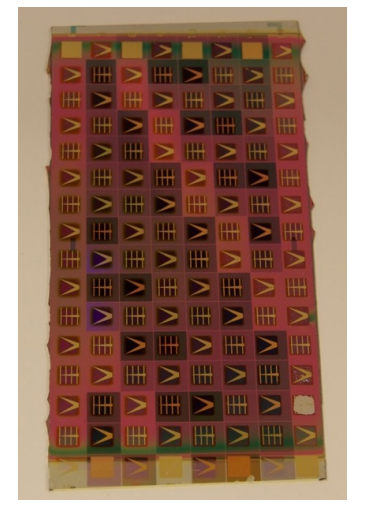

(a)

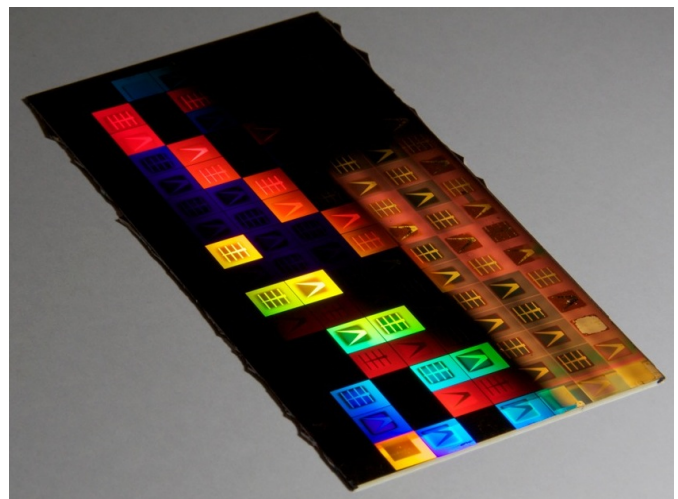

(b)

Figure 6.7. Photographs of fully fabricated solar cells over nanopatterned substrates. The two photographs are of the same array; the second was taken under an angle with illumination.

illumination, so that the periodicity of the square arrays is clearly visible. The 11 nanopatterns chosen are:

1. Flat reference cell

2. $100 \mathrm{~nm}$ wide, $700 \mathrm{~nm}$ pitch

3. $125 \mathrm{~nm}$ wide, $700 \mathrm{~nm}$ pitch

4. $150 \mathrm{~nm}$ wide, $700 \mathrm{~nm}$ pitch

5. $175 \mathrm{~nm}$ wide, $700 \mathrm{~nm}$ pitch

6. $100 \mathrm{~nm}$ wide, $500 \mathrm{~nm}$ pitch

7. $125 \mathrm{~nm}$ wide, $500 \mathrm{~nm}$ pitch

8. $150 \mathrm{~nm}$ wide, $500 \mathrm{~nm}$ pitch

9. $175 \mathrm{~nm}$ wide, $500 \mathrm{~nm}$ pitch

10. $100 \mathrm{~nm}$ wide, $700 \mathrm{~nm}$ pitch, staggered array

11. $150 \mathrm{~nm}$ wide, $700 \mathrm{~nm}$ pitch, staggered array 
After overcoating with $\mathrm{Ag}$, the smallest diameter particles increase in size to approximately $200 \mathrm{~nm}$, and the largest to $290 \mathrm{~nm}$. SEM images of the different patterns after overcoating with Ag are shown in Fig. 6.8, and of the staggered arrays in Fig. 6.9.

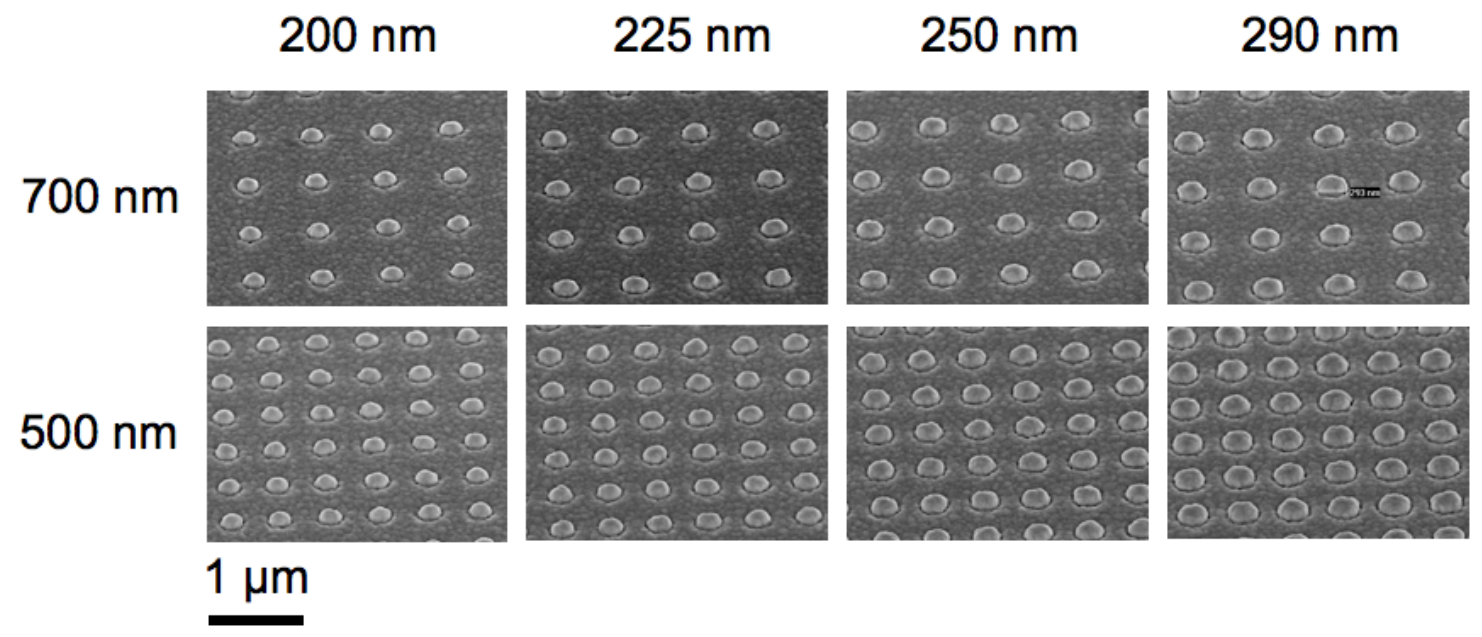

Figure 6.8. SEM images of Ag-coated square periodic arrays with varying pitch and diameter.
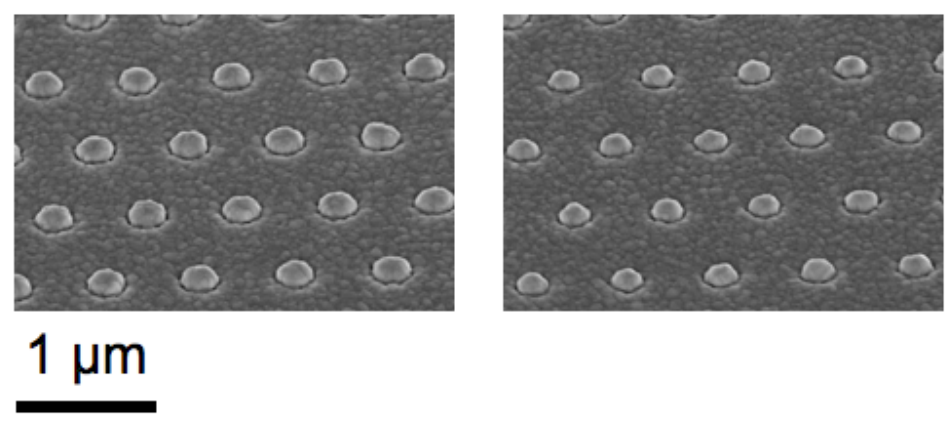

Figure 6.9. SEM images of Ag-coated staggered periodic arrays. The pitch within each row is $700 \mathrm{~nm}$, and the nanoparticles are $250 \mathrm{~nm}$ (left) and $200 \mathrm{~nm}$ (right) in diameter.

Since all realistic a-Si:H solar cells are made on randomly roughened texture, we wanted to compare our nanopatterned back reflectors to cells with random texture. We chose to use Asahi U-type glass as a reference, as it is a well-known industry standard glass for photovoltaics with well-characterized roughness. The glass consists 
110

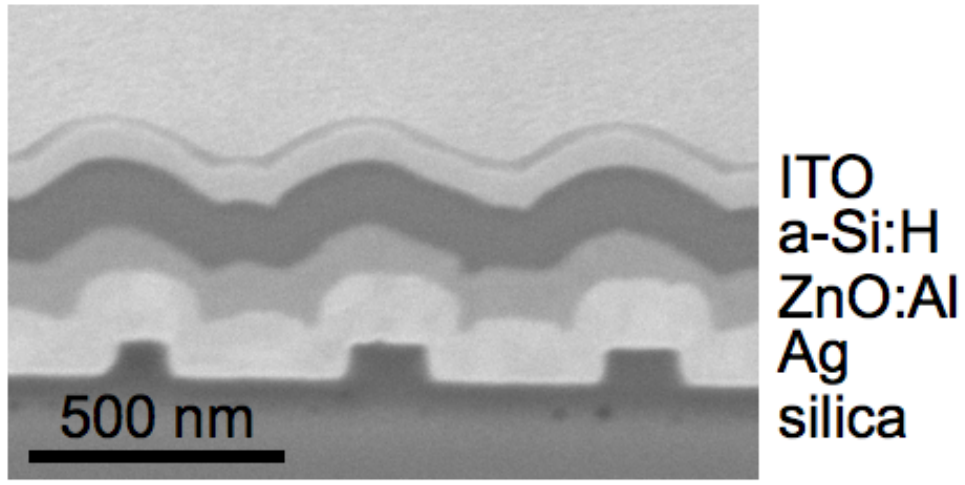

Figure 6.10. FIB cross section of cell with $115 \mathrm{~nm}$ i-layer deposited over nanopattern with $500 \mathrm{~nm}$ pitch. The layers are all conformal over the nanopatterns. The cut was made at a slight angle through the array rather than through the center of the nanoparticles. The absorbing layer is a small fraction of the total device thickness.

of $\mathrm{SnO}_{2}$ F with native texture, and is often used in superstrate- type devices. Here we use only the texture for comparison, by coating over the glass with $\mathrm{Ag}$ and $\mathrm{ZnO}: \mathrm{Al}$ simultaneously to the nanopatterned substrate. Amorphous Si is then deposited over both substrates in parallel.

We deposited cells of two thicknesses: $115 \mathrm{~nm}$ i-layers, and $295 \mathrm{~nm}$ i-layers. Figure 6.10 shows an SEM image of a cross section through a cell with $500 \mathrm{~nm}$ pitch and a $115 \mathrm{~nm}$ i-layer. The individual layers are clearly resolved. The ultrathin a-Si:H layer is conformal to the nanopatterned contacts, with no cracks or voids observed in the layer that could adversely influence the performance [130]. Since each successive layer is conformally deposited, the underlying back contact structure for both the patterned and the randomly textured devices is transferred to the top interface of the cell. Figure 6.11 shows tapping mode AFM scans on the ITO top contact for both the imprint-patterned cell (a) and the randomly textured Asahi sample (b). The imprinted substrate AFM scan reveals the underlying $175 \mathrm{~nm}$ diameter nanopatterns that are imprinted into the sol-gel glass layer, and transferred to the back contact of the cell at a pitch of $500 \mathrm{~nm}$. In contrast, the randomly textured Asahi glass shows an uncorrelated distribution of height variations. 
(a)

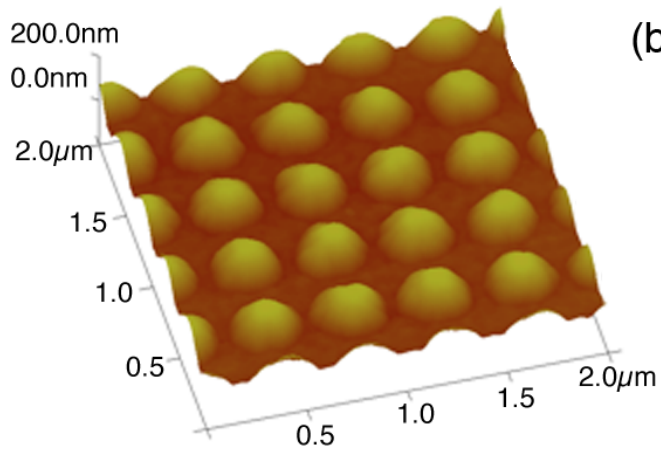

(b)

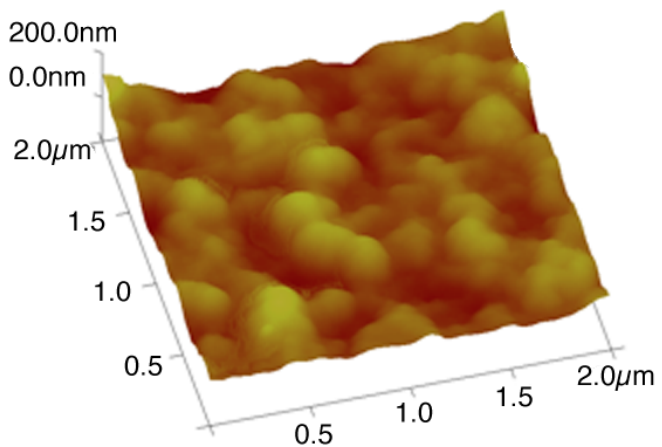

Figure 6.11. Surface topography of nanopatterned and randomly textured solar cells. Tapping-mode AFM images of the top ITO contacts for two of the cells compared in this study. The underlying $\mathrm{Ag} / \mathrm{ZnO}: \mathrm{Al}$ nanostructure is transferred through each layer conformally, so that both the front and back contacts are structured. (a) Patterned cell with $500 \mathrm{~nm}$ pitch, (b) Cell on randomly textured Asahi U-type glass substrate.

\subsection{Comparison of Periodic Structures to Random Textures}

\subsubsection{Efficiency Measurements}

Figure 6.12 shows current density/voltage (J-V) measurements taken under $100 \mathrm{~mW}$ $\mathrm{cm}^{-2}$ AM1.5 illumination for the $295 \mathrm{~nm}$ i-layer (Fig. 6.12(a)) and $115 \mathrm{~nm}$ i-layer (Fig. 6.12(b)) thick cells, all for cells with a plasmonic scatterer diameter of 250 nm. Tables 6.1 and 6.2 show summaries of the full JV measurements on each type of pattern, with the standard deviations shown in parentheses. There is no standard deviation given for $\mathrm{J}_{\mathrm{sc}}$, as these values were determined from integrating the EQE spectrum and weighting by the AM1.5 spectrum, which is often an underestimate of the real $J_{\mathrm{sc}}$.

In Fig. 6.12(a), data for 500 and $700 \mathrm{~nm}$ pitch are shown together with the flat reference. In Fig. 6.12(b), data for the randomly textured Asahi glass cells are also shown. No Asahi reference was fabricated for the cells with $295 \mathrm{~nm}$ i-layer thickness. 


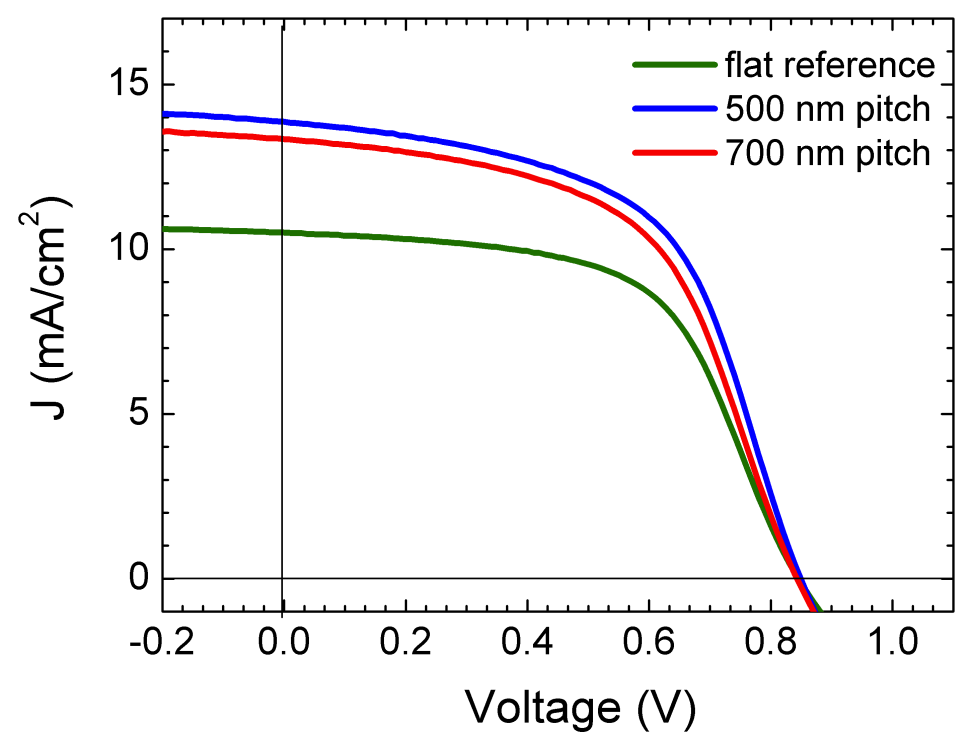

(a) $295 \mathrm{~nm}$ intrinsic layer thickness

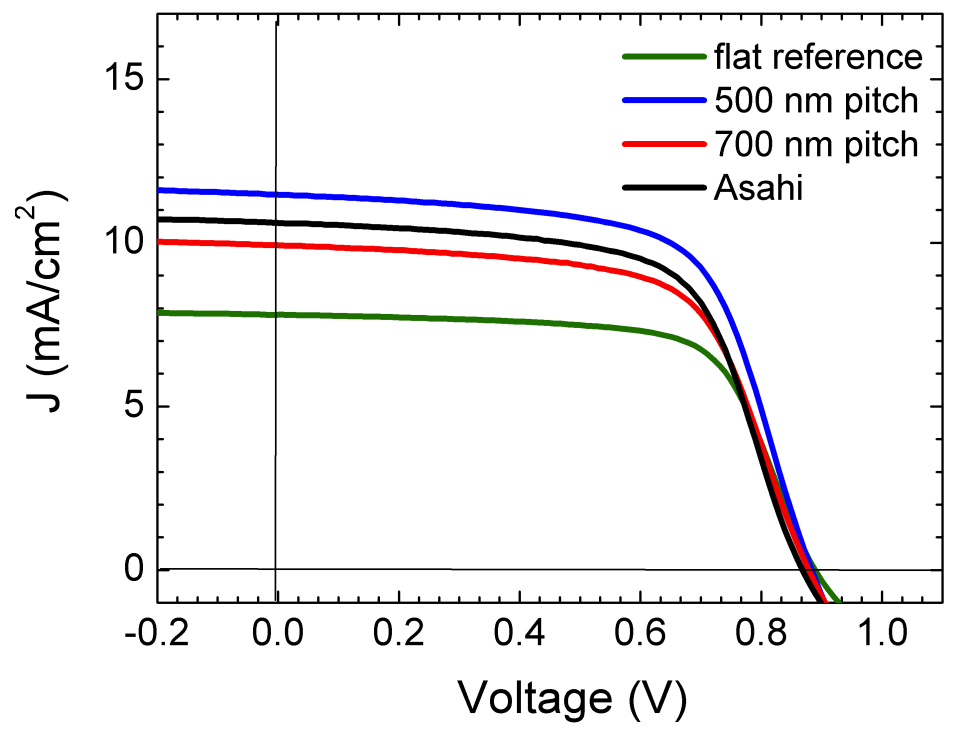

(b) $115 \mathrm{~nm}$ intrinsic layer thickness

Figure 6.12. Electrical measurements on plasmonic solar cells. Data are shown for a-Si:H with two different intrinsic layer thicknesses. (a) intrinisic a-Si:H thickness of $295 \mathrm{~nm}$ and (b) intrinsic a-Si:H thickness of $115 \mathrm{~nm}$. Curves are shown for square grid patterns of $250 \mathrm{~nm}$ diameter plasmonic scatterers at pitches of 500 and $700 \mathrm{~nm}$, the flat reference cell, and (in (b)) the randomly textured Asahi cell. 
113

\begin{tabular}{|c|c|c|c|c|c|}
\hline Diameter $(\mathrm{nm})$ & Pitch $(\mathrm{nm})$ & $\mathrm{V}_{\mathrm{oc}}(\mathrm{V})$ & $\mathrm{FF}$ & $\mathrm{J}_{\mathrm{sc}}\left(\mathrm{mA} / \mathrm{cm}^{2}\right)$ & Eff.(\%) \\
\hline flat & & $0.844(0.004)$ & $0.58(0.01)$ & 10.5 & 5.14 \\
200 & 700 & $0.847(0.005)$ & $0.56(0.01)$ & 12.78 & 6.06 \\
225 & 700 & $0.846(0.009)$ & $0.56(0.01)$ & 12.8 & 6.06 \\
250 & 700 & $0.843(0.01)$ & $0.55(0.002)$ & 13.34 & 6.19 \\
200 & 500 & $0.850(0.007)$ & $0.56(0.007)$ & 13.13 & 6.25 \\
225 & 500 & $0.848(0.005)$ & $0.56(0.010)$ & 13.23 & 6.28 \\
250 & 500 & $0.848(0.007)$ & $0.55(0.01)$ & 13.85 & 6.58 \\
290 & 500 & $0.846(0.01)$ & $0.55(0.01)$ & 13.52 & 6.29 \\
\hline
\end{tabular}

Table 6.1. Summary of JV measurements on cells with $295 \mathrm{~nm}$ thick intrinsic layers. The standard deviations are shown in parentheses.

For the $295 \mathrm{~nm}$ i-layer cells, the $\mathrm{V}_{\text {oc }}$ is in the $840-850 \mathrm{mV}$ range for all devices measured, with standard deviations from 5 to $10 \mathrm{mV}$, indicating that there is no significant difference in semiconductor and contact quality across the substrate. The fill factors are comparable across all measured cells as well, in the 0.55-0.56 range with standard deviations of 0.01. Data taken for several Ag particle diameters $(200,225$, 250, $290 \mathrm{~nm}$ ) all show similar $\mathrm{J}_{\mathrm{sc}}, \mathrm{V}_{\mathrm{oc}}$, and fill factor characteristics, as noted in Table 6.1, with a slight increase in performance for larger diameter scatterers. However, a higher $\mathrm{J}_{\mathrm{sc}}$ is found for cells with a $500 \mathrm{~nm}$ pitch than for cells with a $700 \mathrm{~nm}$ plasmonic scatterer pitch. For the $500 \mathrm{~nm}$ pitch samples, $\mathrm{J}_{\mathrm{sc}}$ improves by $27 \%$ compared to the flat cell. The highest efficiency recorded among cells with the $295 \mathrm{~nm}$ i-layer thick a-Si:H layer $(\eta=6.6 \%)$ was found for a plasmonic scatterer pitch of $500 \mathrm{~nm}$ and a diameter of $250 \mathrm{~nm}$.

For the thinner cells (Fig. 6.12(b)), $\mathrm{V}_{\text {oc }}$ is increased in comparison to the thicker cells, to around $880-890 \mathrm{mV}$, with standard deviations around $20 \mathrm{mV}$. We attribute this improvement to the decreased bulk recombination in thin layers demonstrating, as mentioned previously, an additional important advantage of the use of thin active layers aside from the reduced costs. The fill factors are higher as well, around 0.65 with 0.01 standard deviation. The difference between the diameters is very slight, 
114

\begin{tabular}{|c|c|c|c|c|c|}
\hline Diameter $(\mathrm{nm})$ & Pitch $(\mathrm{nm})$ & $\mathrm{V}_{\mathrm{oc}}(\mathrm{V})$ & $\mathrm{FF}$ & $\mathrm{J}_{\mathrm{sc}}\left(\mathrm{mA} / \mathrm{cm}^{2}\right)$ & Eff.(\%) \\
\hline flat & & $0.890(0.01)$ & $0.68(0.01)$ & 7.86 & 4.76 \\
200 & 700 & $0.867(0.028)$ & $0.65(0.008)$ & 10.2 & 5.75 \\
225 & 700 & $0.884(0.028)$ & $0.66(0.011)$ & 10.57 & 6.17 \\
250 & 700 & $0.882(0.0024)$ & $0.65(0.010)$ & 10.2 & 5.85 \\
290 & 700 & $0.882(0.028)$ & $0.65(0.027)$ & 10.2 & 5.85 \\
200 & 500 & $0.885(0.007)$ & $0.65(0.008)$ & 11.2 & 5.85 \\
225 & 500 & $0.877(0.019)$ & $0.63(0.034)$ & 11.8 & 6.52 \\
250 & 500 & $0.883(0.001)$ & $0.64(0.015)$ & 11.6 & 6.56 \\
290 & 500 & $0.886(0.001)$ & $0.66(0.004)$ & 11.2 & 6.55 \\
\hline Asahi & & $0.869(0.008)$ & $0.64(0.008)$ & 10.8 & 6.00 \\
\hline
\end{tabular}

Table 6.2. Summary of JV measurements on cells with $115 \mathrm{~nm}$ thick intrinsic layers. The standard deviations are shown in parentheses.

but there is a more substantial difference between the two pitches in $\mathrm{J}_{\mathrm{sc}}$, as shown in Table 6.2. The $500 \mathrm{~nm}$ pitch, $250 \mathrm{~nm}$ diameter samples now show an increase in $\mathrm{J}_{\mathrm{sc}}$ of $46 \%$ over the $160 \mathrm{~nm}$ thick flat cell. The best cell measured again had 250 $\mathrm{nm}$ diameter plasmonic scatterers and $500 \mathrm{~nm}$ pitch. This cell has an efficiency of $6.6 \%$, which is similar to the maximum efficiency found for the thick cell. While $\mathrm{J}_{\mathrm{sc}}$ is lower in the thin cells, the increased $V_{\text {oc }}$ and fill factor cause the overall efficiency to remain the same between the two thicknesses. This demonstrates conclusively that plasmonic back reflectors can be used to maintain efficiency while scaling to thinner solar cells. The $\mathrm{J}_{\mathrm{sc}}$ of the $500 \mathrm{~nm}$ patterned cell is improved by $50 \%$ compared to the flat reference cell.

Remarkably, Fig. 6.12(b) also shows that $\mathrm{J}_{\mathrm{sc}}$ for the patterned cell with $500 \mathrm{~nm}$ pitch is significantly larger than for the randomly textured cell with Asahi-U type of texture. Because the nanopatterned cells and the randomly textured Asahi sample both have comparable fill factor and $\mathrm{V}_{\text {oc }}$, we can exclude a difference in semiconductor quality as an explanation for the improved $\mathrm{J}_{\mathrm{sc}}$. We conclude that light trapping in the $500 \mathrm{~nm}$ pitch patterned cell is more efficient than in the randomly textured sample. 


\subsubsection{External Quantum Efficiency Measurements}

To further study the nature of the photocurrent enhancement, we measured external quantum efficiency (EQE) spectra, defined as the number of collected charge carriers per incident photon, using a Xenon lamp under light bias corresponding to approximately one sun illumination and $0 \mathrm{~V}$ bias. Figure 6.13 shows the measured EQE spectra for the two thicknesses explored in this study. We note a slight increase in

photocurrent on the blue side of the band for the $500 \mathrm{~nm}$ pitch cell, from 350 to $550 \mathrm{~nm}$, which we attribute to improved antireflective properties of the corrugated top surface of the cell, and will be discussed further in section 6.8. For both thicknesses, the patterned cells show a large photocurrent enhancement on the red side of the band. These peak positions are reproduced clearly in all cells with the same nanopattern pitch, as shown in Fig. 6.14.

Focusing on the thinner cells shown in Fig. 6.13(b), the primary photocurrent enhancement occurs in the 550 to $800 \mathrm{~nm}$ spectral range. While the EQE of both the $500 \mathrm{~nm}$ and the $700 \mathrm{~nm}$ pitch cells exceeds that of the flat reference cell, there is a pronounced difference between the spectra. The cell on the randomly textured Asahi substrate has a very smooth EQE response, while the patterned devices exhibit peaked features in the EQE curve, of which the peak wavelengths are reproducible for each pitch. Notably, the EQE of the $500 \mathrm{~nm}$ pitch cell exceeds that of the randomly textured Asahi sample in the 550 - $650 \mathrm{~nm}$ spectral range where there is significant power in the solar spectrum. A higher resolution measurement of the EQE from the $500 \mathrm{~nm}$ pitch cells and the Asahi texture cells is shown in Fig. 6.15, as measured using a supercontinuum laser light source rather than a halogen lamp. From 650 $\mathrm{nm}$ to $800 \mathrm{~nm}$ the features in the spectra of the nanopatterned cell sharpen and alternately exceed and fall below the curve for the randomly textured Asahi cell.

\subsection{Angle-Resolved Photocurrent}

To further investigate the nature of the light trapping mechanism for the nanopatterned and randomly textured Asahi glass samples, we measured EQE spectra as a 


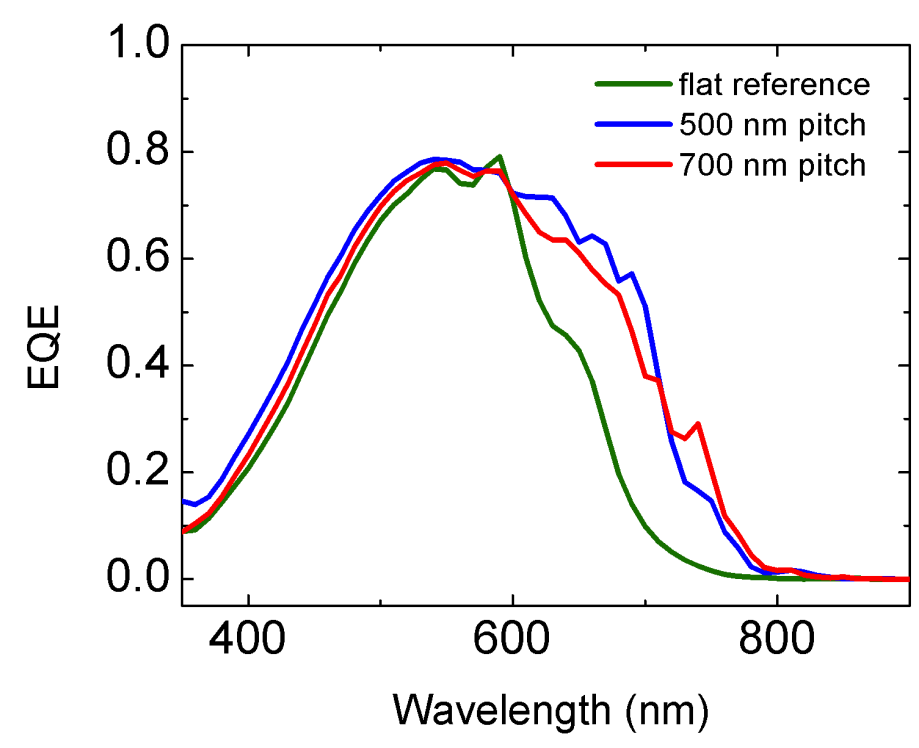

(a) $295 \mathrm{~nm}$ intrinsic layer thickness

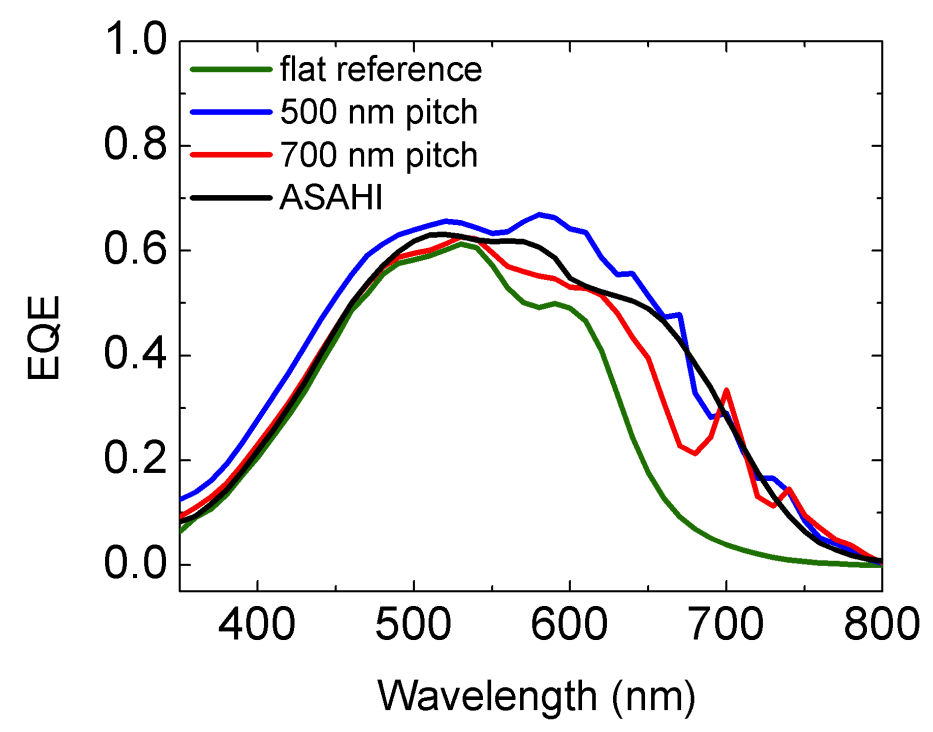

(b) $115 \mathrm{~nm}$ intrinsic layer thickness

Figure 6.13. External quantum efficiency spectra of nanopatterned and randomly textured cells from measurement and simulation. EQE spectra are shown in (a) for cells of thickness $160 \mathrm{~nm}$, under one sun illumination at $0 \mathrm{~V}$ bias. The primary enhancement in photocurrent over the flat reference cell occurs from 550 to $800 \mathrm{~nm}$. The $500 \mathrm{~nm}$ pitch cell shows higher EQE than the randomly textured Asahi cell. 


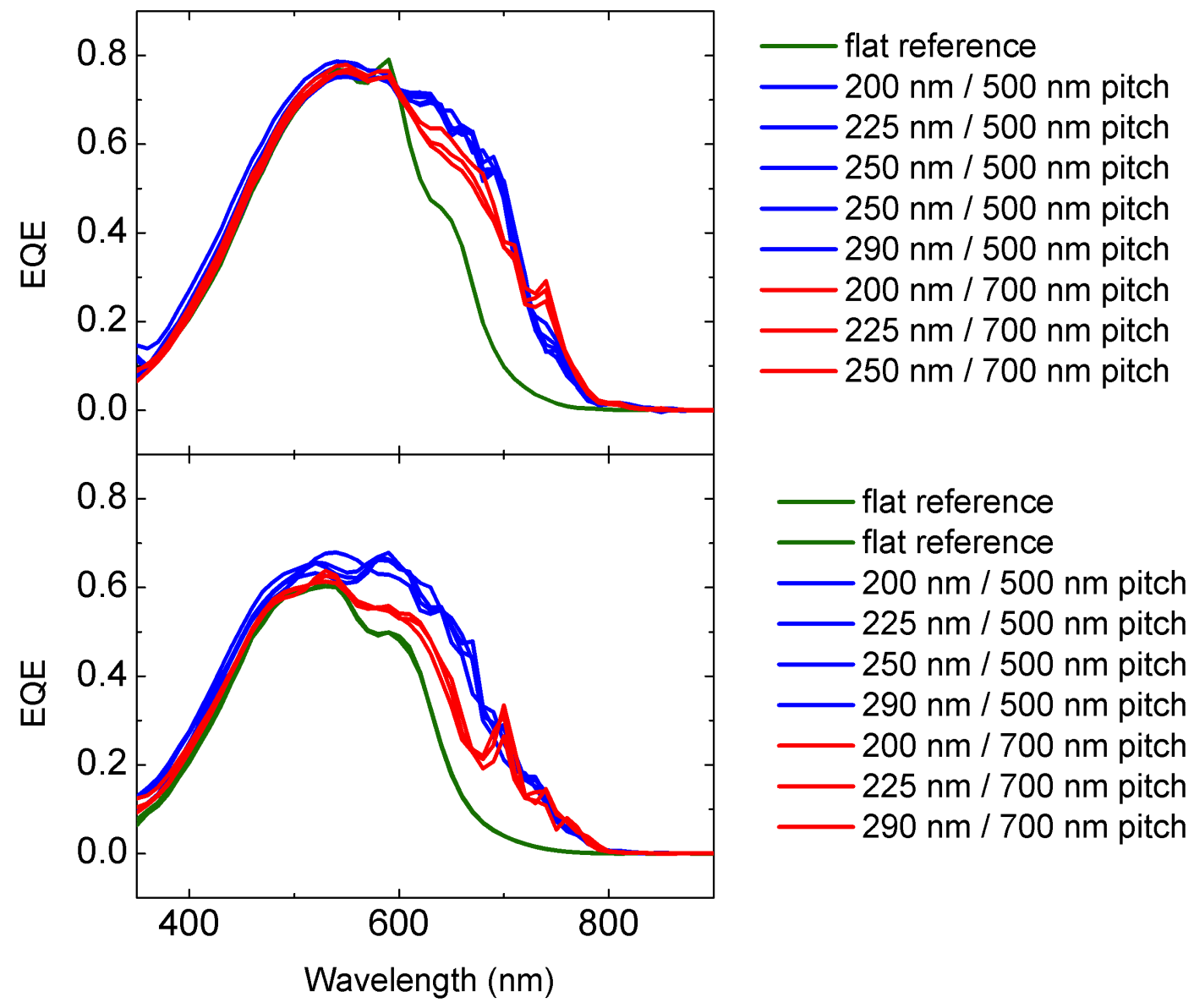

Figure 6.14. EQE measurements on several different cells with the same nanopattern, indicating that the observed features on the red side of the spectrum are both repeatable and correlated with pitch. Top: cells with 295 nm i-layer thicknesses, bottom: cells with 115 nm i-layer thicknesses. 


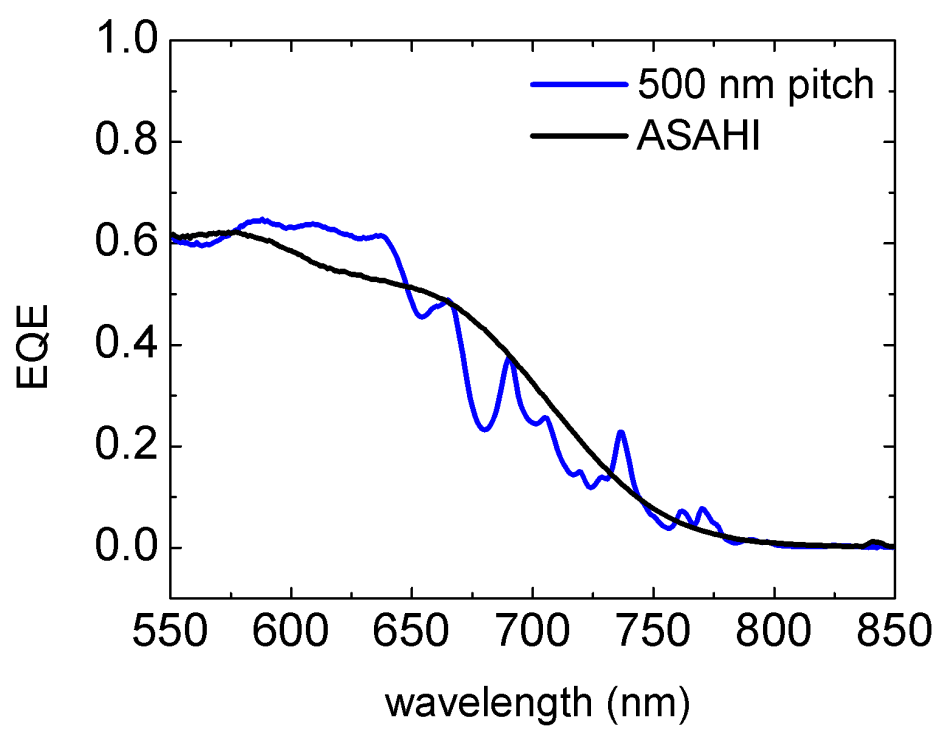

Figure 6.15. EQE measurements on cells with $500 \mathrm{~nm}$ pitch periodic nanopattern and Asahi texture, measured with a supercontinuum laser light source rather than a halogen lamp for higher resolution.

function of incident angle for the thinner cells. Angle-resolved EQE measurements were performed using a supercontinuum laser source in combination with a monochromator with a passband of $3 \mathrm{~nm}$ and a sample stage providing eucentric rotation about the point of illumination. The illumination was focused to a spot diameter of approximately $500 \mu \mathrm{m}$ at low numerical aperture. Fig. 6.16 shows intensity maps of these angle-resolved EQE spectra, for angle of incidence between -45 and +45 from the substrate normal and for a range of illumination wavelengths from $550 \mathrm{~nm}$ to 850 $\mathrm{nm}$, for the randomly textured Asahi cell (Fig. 6.16(a)) and the $500 \mathrm{~nm}$ pitch cell (Fig. 6.16(b)). The EQE curves at normal incidence were found to agree well with the measurements shown in Fig. 6.13.

Some variation with angle of incidence is expected for all cells due to the angular response of the anti-reflection coating present on the cells. However, while it is evident that the randomly textured Asahi cell has a relatively isotropic spectral response to angle of incidence, the $500 \mathrm{~nm}$ pitch cell exhibits more complex behavior. The enhanced EQE for the patterned cell in the 550 - $650 \mathrm{~nm}$ range is observed, and can be seen to extend to at least +/- 20 degrees. From 650 to $800 \mathrm{~nm}$ the spectral 


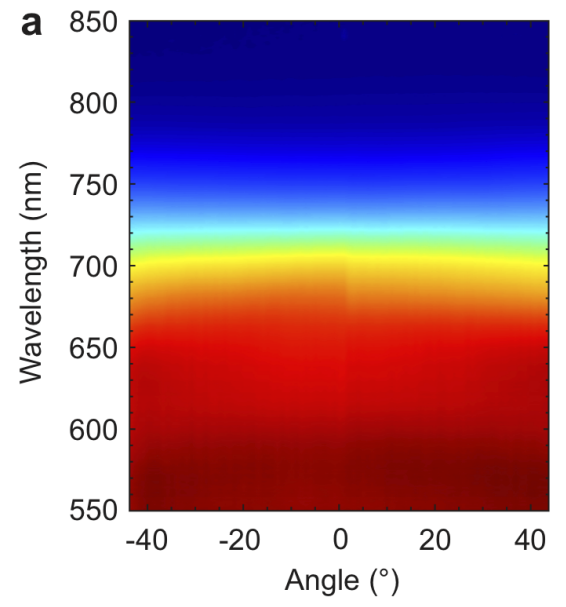

(a) Asahi

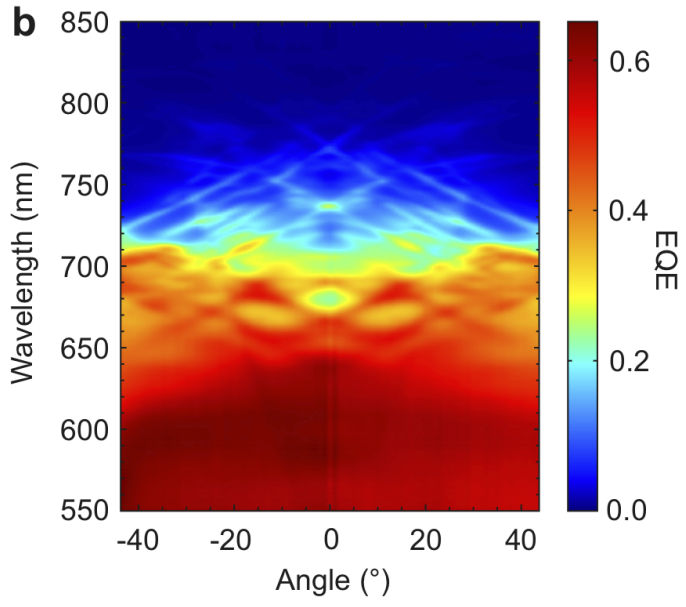

(b) $500 \mathrm{~nm}$ pitch

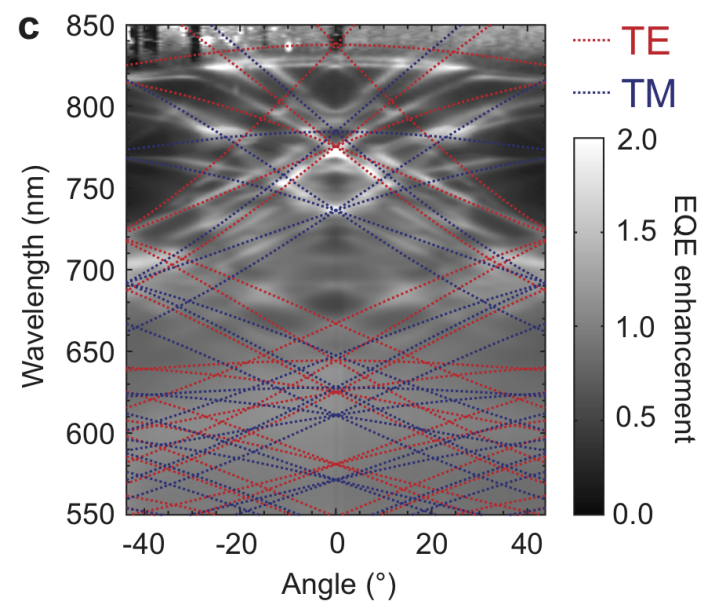

(c) EQE enhancement with mode calculations

Figure 6.16. Angle-resolved photocurrent spectroscopy. Measured EQE versus incident wavelength and incident angle for cells with $115 \mathrm{~nm}$ thick i-layers. The EQE enhancement for the nanopatterned sample, the ratio of $6.16(\mathrm{~b})$ to $6.16(\mathrm{~b})$, is shown in $6.16(\mathrm{c})$; the calculated folded-zone dispersion diagram of the lowest-order TE and TM modes is superimposed. 
photocurrent features become sharper and show a stronger angular dependence. The dispersive features measured in Fig. 6.16(b) are clear evidence of coupling between light scattered by the grating and the guided modes supported by the high-index a-Si:H layer of the solar cell.

We then calculated the dispersion curves for the guided modes present in a representative planar device with $500 \mathrm{~nm}$ periodicity. The calculations were performed using the experimentally determined layer thicknesses and optical constants. In Fig. 6.16(c), branches corresponding to the TE and TM modes with the highest modal overlap with the a-Si:H are shown, folded back to the angular range of interest by taking diffraction by the two-dimensional periodic structure into account. The curves are superimposed over an intensity map of the relative enhancement of the EQE of the patterned cell compared to the randomly textured Asahi cell. Clearly, the pattern of dispersive bands observed in the EQE measurements agrees well with the calculated mode dispersion. In particular, the bands of enhanced absorption in the $700-850 \mathrm{~nm}$ range are well explained by the model, including the crossings at $740 \mathrm{~nm}$ and $770 \mathrm{~nm}$. Deviations between measurement and calculation are attributed to small differences in optical constants and the fact that the calculation does not include surface corrugation and associated mode coupling. Additional spectral features observed in the EQE spectra could possibly be associated with absorption due to local field enhancements in the nanostructures. The measurement clearly shows that for these wavelengths, coupling to modes guided by the a-Si:H layer is responsible for increased absorption in the cell. Calculations show a high density of modes in the spectral range 550 - 650 $\mathrm{nm}$ where the EQE of the patterned cell exceeds that of the randomly textured Asahi substrate. The fact that in this region no sharp features are observed in the measurements is due to the large number of modes present, which are strongly broadened because of large absorption of a-Si:H in this spectral region. Although similar modes may be observed for patterned dielectric particles, the scattering cross section of the metal nanostructures increases coupling to the guided modes, further enhancing the photocurrent. 


\subsection{Conclusion}

In summary, we have demonstrated a-Si:H solar cells with efficient light trapping by a nanoengineered plasmonic back reflector. Light is scattered strongly from the nanopatterned metal back contact into guided modes of the cell, enhancing the photocurrent for a given cell thickness by enhancing the coupling to the modes. Similarly, the same photocurrent can be achieved at a reduced cell thickness, with a concomitant increase in open circuit voltage (and potential for increased long-term stability). We demonstrate that nanoengineered cells show light trapping that is enhanced beyond that of a randomly textured cell. Furthermore, the cells are fabricated via a low-cost and scalable nanoimprint method. We now turn our attention to modeling of these devices, for both improved understanding of the light trapping mechanism and optimization of the nanostructures and patterns. 


\section{Chapter 7}

\section{Simulation of Ultrathin-Film a-Si:H Solar \\ Cells: Comparison to Experimental Measurements and Optimization}

\section{$7.1 \quad$ Introduction}

With increased computational power in recent years, electromagnetic modeling has become an important tool for optimizing the shape and arrangement of nanostructures for enhanced absorption in solar cells. A variety of simulation techniques have been utilized, including finite element, rigorous coupled wave analysis, transfer matrix methods, and semiempirical treatments, particularly within the last three years $[23$, $24,100,131,132,133]$.

Given realistic cell depositions, however, the actual shape of the fabricated device can differ from those proposed in simulation. Finite element models offer more flexibility than other simulation methods, as they do not require assumptions of periodicity or regularity [72]. Conformal structures and randomly textured surfaces can be modeled accurately, albeit with more computational power than other methods that make simplifying assumptions. Here we discuss electromagnetic modeling and optimization of cells using experimental cross sections and AFM data as a guide to modeling realistic cell architectures. We include modeling of all necessary spacer layers and optically relevant contacts. The excellent agreement between experimental devices and simulated absorption allows us to both explore a wide parameter space and to study the details of the physics in light trapping. 


\subsection{Simulation of Fabricated Cells}

Finite element models make two assumptions that connect the simulation to experimental devices: the geometrical layout of all of the layers, and the optical constants of the materials in the layers. To model the optical properties of each layer accurately, we use the complex refractive index measured from ellipsometry (Fig. 7.1). The optical properties of ITO, ZnO:Al, and a-Si:H can vary based on deposition conditions, so it is particularly important to use optical data from the same deposition chamber as the experimental devices. For Ag, we use a Lorentz-Drude fit to Palik's Ag measurements, following the method of Rakic [78, 79]. Even though these simulations are done at single wavelengths, they are done in the time domain, which requires full treatment of the dispersion of each material. While this is critical for Ag, Fig. 7.1 indicates that the ITO and $\mathrm{ZnO}: \mathrm{Al}$ have relatively constant optical properties with wavelength, and that a-Si:H can be approximated as having a constant index within each single wavelength simulation. Of course the refractive index is set differently for each wavelength. While the refractive index of a-Si:H does vary with hydrogen content, and thus varies within the profile of the cell in the doped regions and interfacial regions, that difference in the optical properties is neglected here and a-Si:H is assumed to have uniform refractive index across the whole region.

Figure 7.2 shows a schematic version of a nanopatterned cell as simulated. The

size and shape of the Ag nanoparticles are estimated based on the AFM profiles of the Ag-coated contacts, and the conformal coating of each layer is approximated from cross section SEM images. For periodic cases we apply periodic boundary conditions in the $\mathrm{x}$ - and $\mathrm{y}$-directions, and perfectly matched layers in the z-direction. The thickness of the layer used for a-Si:H is the total a-Si:H layer thickness, including the $\mathrm{n}$ and $\mathrm{p}$ doped regions. While these layers are likely optically dead and contribute little to the photocurrent, the thickness of the entire region determines the presence of modes.

Simulations can also be done using imported AFM or SEM data, although this increases simulation complexity. To simulate the randomly textured layers, AFM scans 


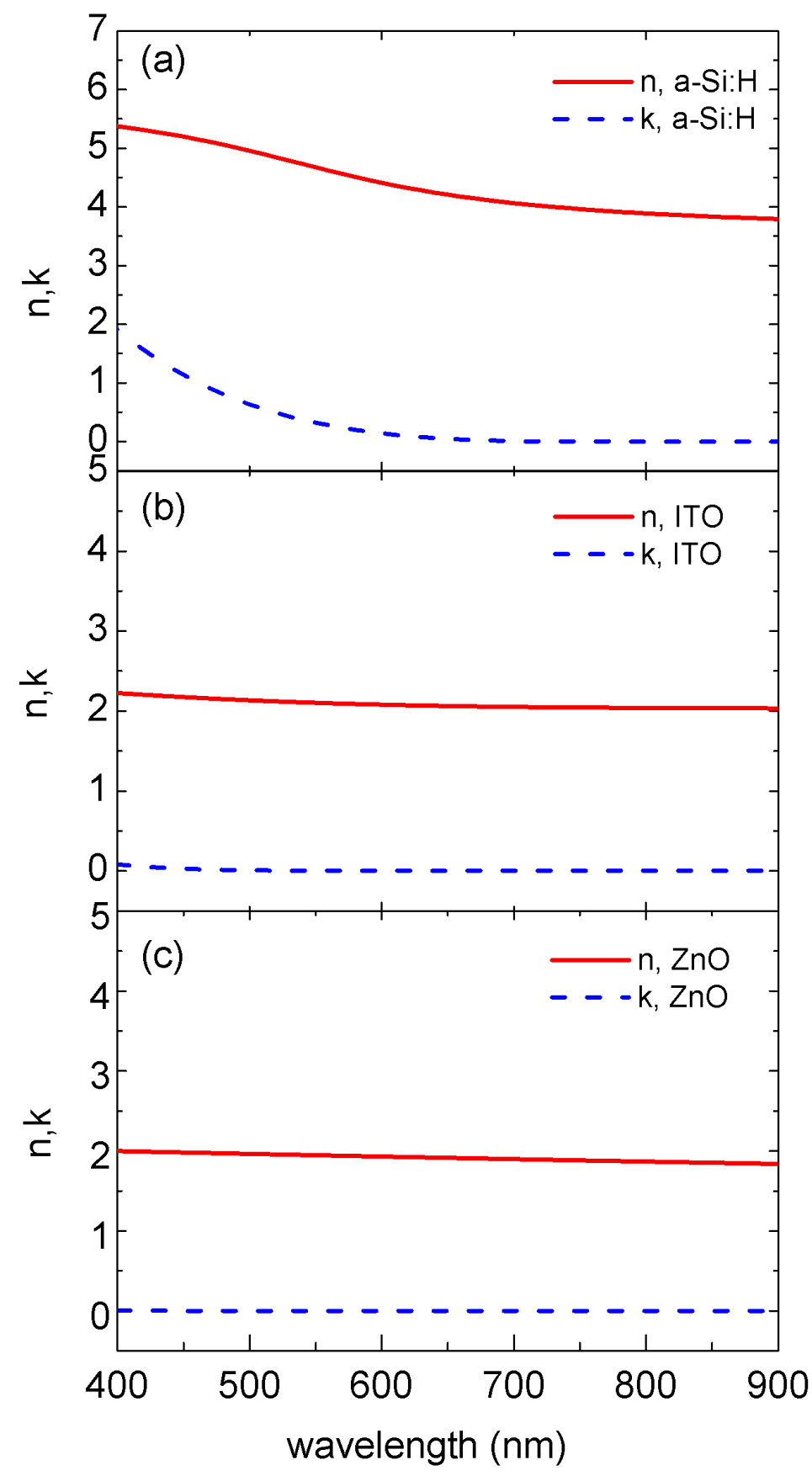

Figure 7.1. Complex refractive index for each of the layers, as measured with ellipsometry. 


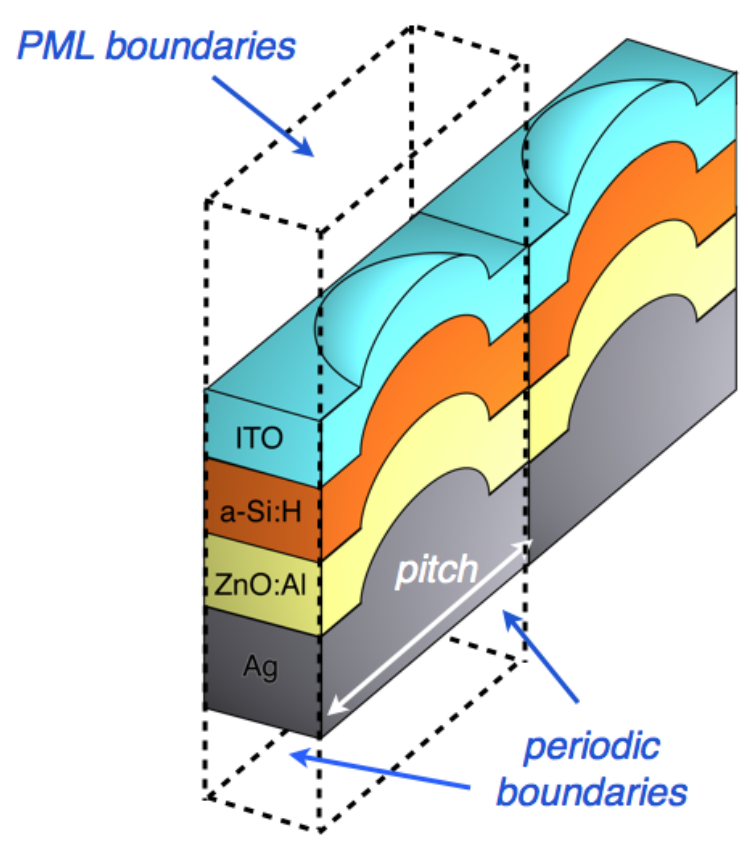

Figure 7.2. Schematic of conformal nanopatterned cell as modeled in simulation.

of randomly textured surfaces were imported directly into the simulation software. Figure 7.3(a) shows an AFM scan of a $10 \mu \mathrm{m}$ x $10 \mu \mathrm{m}$ region of Ag-coated Asahi Utype glass. Rather than make assumptions about conformal broadening on a complex surface, the texture was propagated identically in each layer of the simulation. For accurate meshing of these surfaces the $10 \mu \mathrm{m} \times 10 \mu \mathrm{m}$ region is prohibitively large, and so simulations were done using a subset of the texture, shown in Fig. 7.3(b). Periodic boundary conditions were assumed on the $2 \mu \mathrm{m} \times 2 \mu \mathrm{m}$ region to avoid reflections from the plane wave source at the boundaries. Although this assumption could introduce error into the assumption of randomness, the standard deviation of absorption was calculated using a statistical bootstrapping method, and verified to be under $5 \%$ at all wavelengths simulated.

Figure 7.4 shows the comparison between the experimental cells on nanopatterned substrates (Fig. 7.4(a)) and the simulated cells (Fig. 7.4(b)). The agreement between the two is generally good, particularly for the Asahi pattern. As the simulation technique is only optical and does not account for carrier collection, some features may be magnified in simulation that are not observed as strongly experimentally. Other 


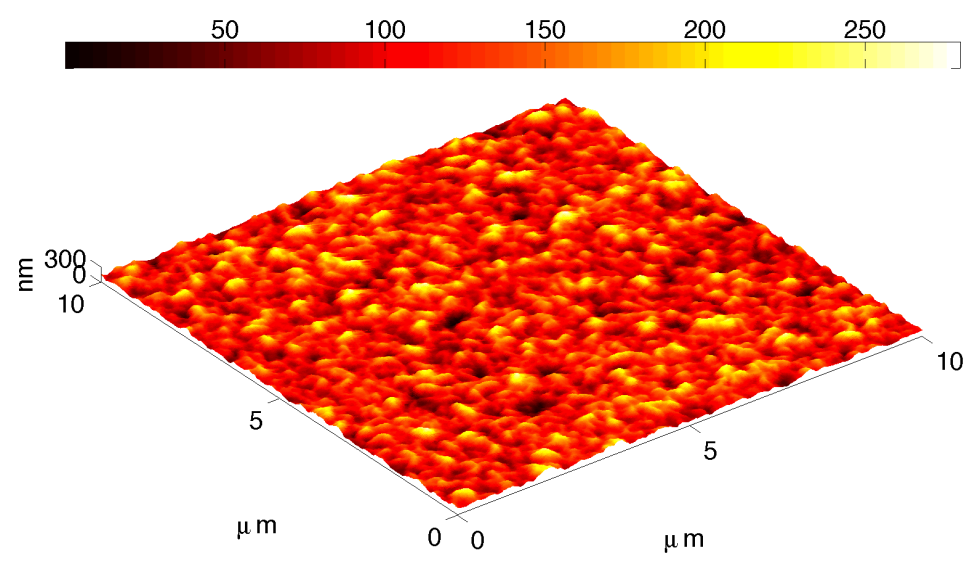

(a) Full scan

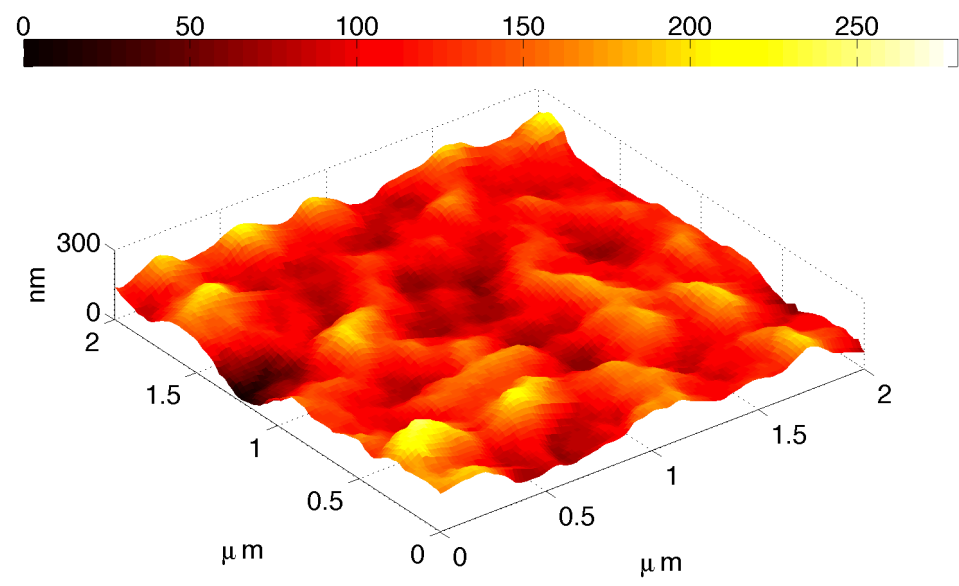

(b) Subset used for simulation

Figure 7.3. AFM images of Ag-coated Asahi U-type glass used for simulation. 
128

differences between the two may be due to the assumed geometries or variations in the optical constants, particularly in the a-Si:H layer which varies with the doping and hydrogen content. The agreement between simulation and experiment is generally less accurate on the blue side of the spectrum, which is reasonable considering that the simulation assumes every photon produces one electron, which is collected. At the shorter wavelengths the collection process is inefficient, and the number of collected electrons will be a lower percentage of the total generated carriers.

\subsection{Understanding Light Trapping in Periodic Cells}

\subsubsection{Absorption across solar spectrum}

One of the advantages of this simulation technique is that the fields are calculated explicitly everywhere in the simulation. This allows us to make maps of the absorption at different cross sections of the data set, and visualize the magnitude of the absorption in each of the layers. As explained in Chapter 3, we calculate a photon flux from the electric field magnitude according to Equation 7.1.

$$
\Phi(\lambda)=\frac{1}{2 \hbar} \int \epsilon^{\prime \prime}|E|^{2} d V
$$

We calculate $\Phi$ over the whole structure and use the corresponding index monitor data set to isolate the absorption in the a-Si:H before integrating. This also allows us to calculate the losses in the supporting materials. If we integrate over just the isolated region containing a-Si:H, and assume that every absorbed photon produces one electron, then this is the generation rate $G_{\text {opt }}$. With the additional assumption that every generated electron is collected, and weighted by the solar spectrum, the generation rate can be converted into a maximum possible short circuit current.

To look in more detail at the absorption processes in the cell, we calculated maps of the absorption in the different layers. Figure 7.5 shows maps of $\Phi$ and $G_{o p t}$ on a logarithmic color scale for a cross-section through an example three-dimensional simulation. The Ag particles are $300 \mathrm{~nm}$ in diameter, the pitch is $500 \mathrm{~nm}$, and the 


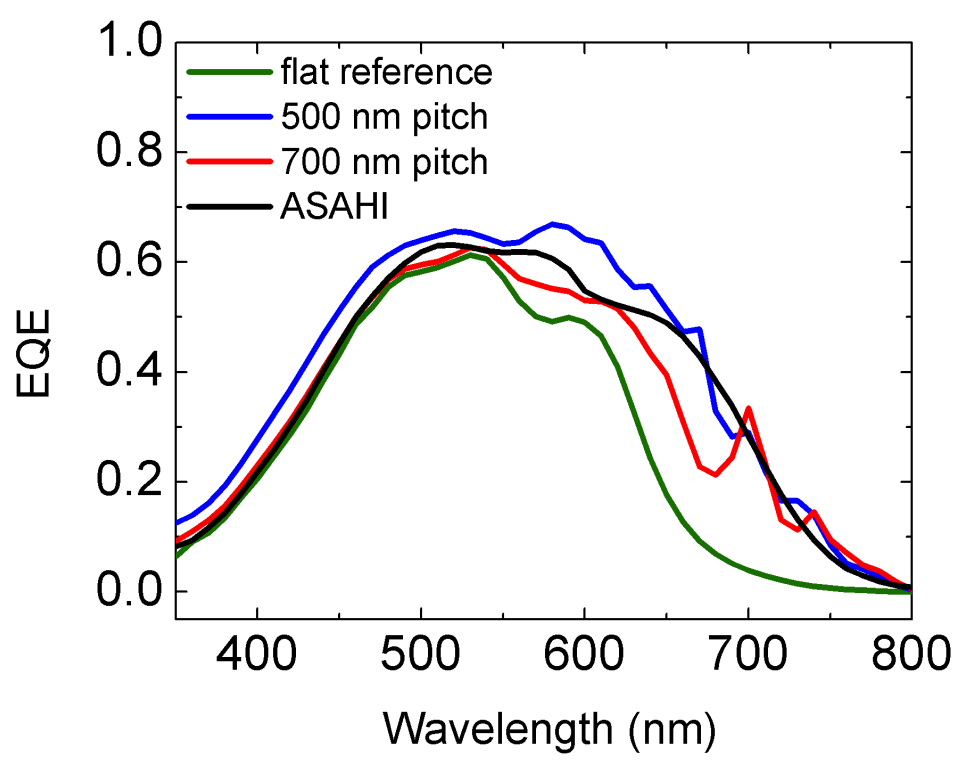

(a) Experiment

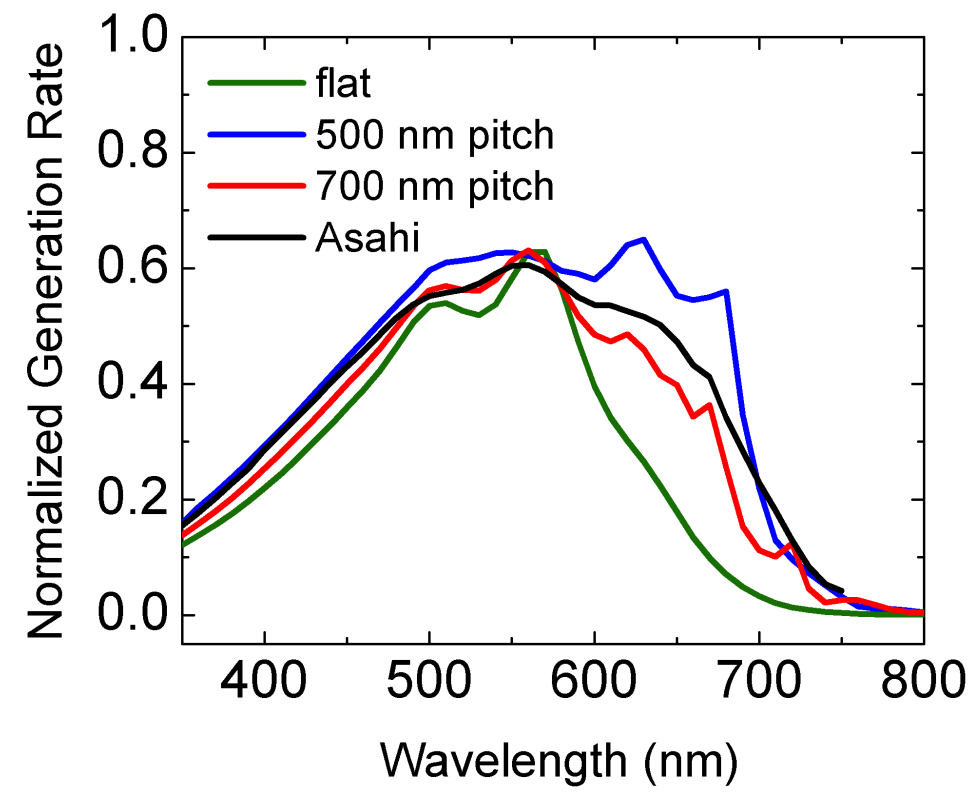

(b) Simulation

Figure 7.4. External quantum efficiency spectra of nanopatterned and randomly textured cells from measurement and simulation. EQE spectra are shown in (a) for cells of thickness $160 \mathrm{~nm}$, under one sun illumination at $0 \mathrm{~V}$ bias. 

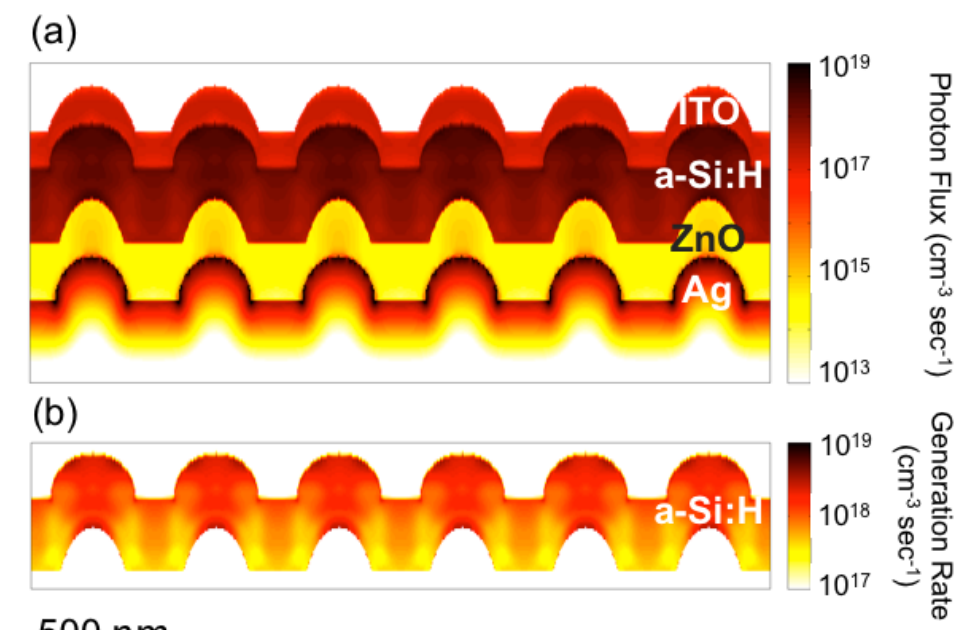

$500 \mathrm{~nm}$

Figure 7.5. Solar spectrum-weighted photon flux maps for periodic cells. (a) All layers of the solar cell shown. (b) Same simulation as (a), but with the a-Si:H layer isolated for better resolution.

intrinsic a-Si:H layer is $115 \mathrm{~nm}$ thick. Figure 7.5(a) shows the solar spectrum weighted $G_{\text {opt }}$ for the wavelength range from 350 to $800 \mathrm{~nm}$ where a-Si:H is active. It is evident from the image that absorption is highest in the semiconductor a-Si:H layer, but that absorption in the ITO is also a significant source of loss. The $\mathrm{ZnO}$ :Al spacer layer on the back has quite low loss, and the skin depth of the Ag is also visible as absorption here is strongest within the first few nanometers.

We can also examine the profiles of the absorption within the active a-Si:H region for information about the mechanisms leading to enhanced absorption. Figure 7.5(b) shows the same data as in Fig. 7.5(a), but here the a-Si:H region is isolated and the color scale is modified to show the spatial inhomogeneity of the absorption. The absorption is strongest in the curved region of the cell directly above the scatterer.

It is informative to break the absorption down by wavelength, to visualize the mechanisms that contribute to absorption in different regions of the solar spectrum. Figure 7.6 show maps of the absorption in a-Si:H at four individual wavelengths. In Fig. 7.6(a), the incident wavelength is $400 \mathrm{~nm}$, and it is evident that the absorption is confined to the top portion of the semiconductor. Any enhanced absorption observed or calculated in this spectral range thus cannot be due to the back contact pattern, 


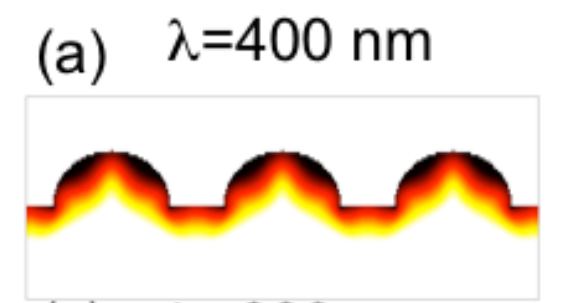

(c) $\lambda=680 \mathrm{~nm}$

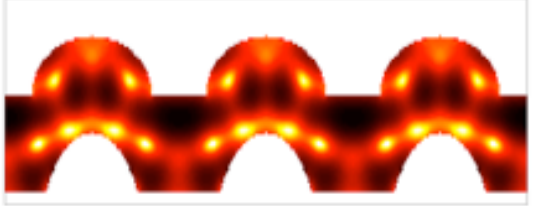

$500 \mathrm{~nm}$ (b) $\lambda=550 \mathrm{~nm}$

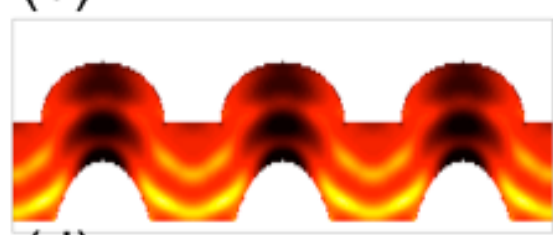

(d) $\lambda=690 \mathrm{~nm}$

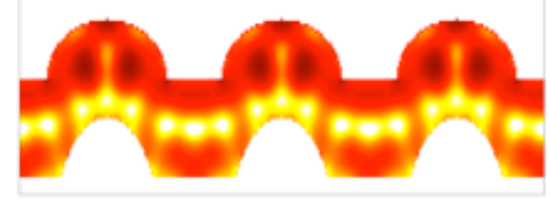

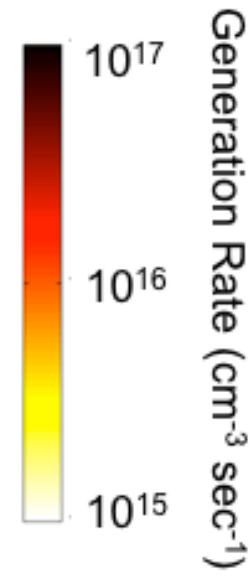

Figure 7.6. Generation rate maps at different wavelengths spanning the solar spectrum.

as the incident light is strongly absorbed before reaching the back. It may, however, result from the front-side curvature of the ITO and a-Si:H. Figure 7.6(b) shows the absorption at $\lambda=550 \mathrm{~nm}$, an intermediate wavelength near the thin-film Fabry-Perot resonance for $160 \mathrm{~nm}$ thick a-Si:H. Figures 7.6(c) and 7.6(d) show the absorption map due to waveguide modes. At $\lambda=680 \mathrm{~nm}$, where there is a peak in the calculated photocurrent in Fig. 7.4, the absorption is very strong, whereas at $\lambda=690 \mathrm{~nm}$ the mode has been pushed into cutoff and the absorption is much weaker.

\subsubsection{Where Are the Losses?}

The losses in each material are shown quantitatively in Fig. 7.7 as a function of wavelength. As seen in Fig. 7.5 the majority of the absorption is beneficial and occurs in the a-Si:H. On the blue side of the spectrum, from 350 to $500 \mathrm{~nm}$, the parasitic absorption occurs entirely in the ITO layer. This confirms the interpretation that on the blue side the a-Si:H layer is optically thick, and the incident sunlight does not interact with the back metal contact. The remaining portion is reflection. This result underscores the importance of alternative top contact designs, with either optimized nanopatterns for reduced reflection (as will be discussed later), or alternatives to ITO such as those from metallic nanowires or arrays [134]. From 500 to $750 \mathrm{~nm}$ the 
132

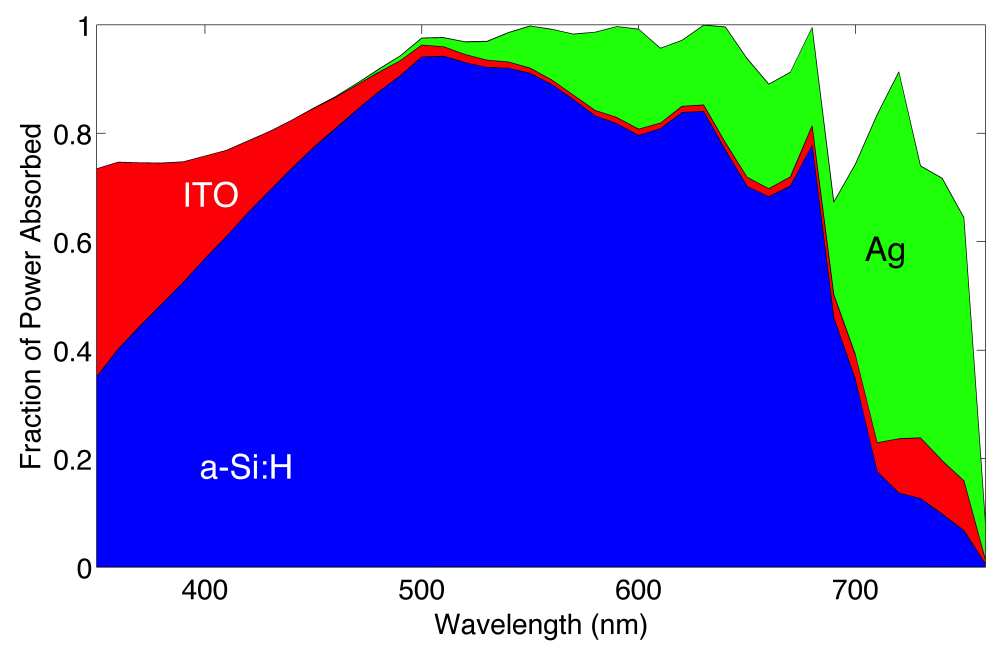

Figure 7.7. Calculated absorption in all of the layers of an example cell with $300 \mathrm{~nm}$ Ag particles, $500 \mathrm{~nm}$ pitch, and $115 \mathrm{~nm}$ thick intrinsic layers.

majority of the parasitic absorption is due to the loss in Ag. To reduce these metal losses, particularly from 500 to $650 \mathrm{~nm}$, we explore the use of other plasmonic metals. Finally we note the sharp peaks in the spectrum attributed to waveguide modes, particularly from 600 to $750 \mathrm{~nm}$; control of the pitch can tune the position of these modes.

\subsubsection{Role of the $\mathrm{ZnO}$ :Al Layer}

The ZnO:Al layer serves a couple of purposes in the device. During growth of n-i-p cells, the elevated temperature of a-Si:H deposition can cause the Ag to diffuse into the a-Si:H, causing defects and trapping within the semiconductor. The ZnO:Al thus acts as a buffer layer, preventing Ag diffusion. A second function is optical: the presence of the ZnO:Al layer shifts the SPP mode significantly. We saw in previous chapters that the SPP mode has poor overlap with the semiconductor layer, and can frequently lead to more parasitic loss than to beneficial photocurrent-generating absorption. By including the $\mathrm{ZnO}$ :Al layer, the dispersion relation of the modes in the cell is significantly changed.

Figure 7.8 shows dispersion relation calculations for a flat layer stack consisting 
of $\mathrm{Ag} /$ varying $\mathrm{ZnO}$ thickness/ $160 \mathrm{~nm}$ a-Si:H/80 nm ITO/air. The $160 \mathrm{~nm}$ a-Si:H corresponds to a $115 \mathrm{~nm}$ i-layer thickness, as used experimentally, with $45 \mathrm{~nm}$ of doped layers. Figure 7.8(a) shows the modes when the $\mathrm{ZnO}: \mathrm{Al}$ is absent, and Fig. 7.8(b) when $10 \mathrm{~nm}$ of $\mathrm{ZnO}: \mathrm{Al}$ is introduced. While the photonic modes look similar, the SPP mode is dramatically changed with $10 \mathrm{~nm}$ of the lower index $\mathrm{ZnO}$ :Al layer. Figure 7.8(c) shows the same calculation for $130 \mathrm{~nm}$ of $\mathrm{ZnO}: \mathrm{Al}$, the real thickness used in the experimental cells. The structure of the photonic modes has changed slightly, and the SPP mode is absent altogether. Further FDTD simulations of the $\mathrm{ZnO}$ :Al layer thickness showed weak dependence on the layer thickness.

\subsubsection{Is the Metal Necessary?}

One of the central issues in understanding the observed light trapping effects is the precise role of the nanostructured metal. The waveguide modes which depend on periodicity will be present so long as the cell has a periodic structure; and the modes themselves are photonic modes rather than surface plasmon polaritons (SPPs). The presence of the $\mathrm{ZnO}: \mathrm{Al}$ spacer layer between the metal and the a-Si:H shifts the SPP mode so that it does not play a role in enhancing absorption. The metal nanostructures do, however, support localized plasmonic modes with high scattering cross sections which can enhance coupling to the waveguide modes of the device.

To examine the role of the metal we first analyzed the somewhat unphysical but useful structure illustrated in Fig. 7.9. The schematics in Fig. 7.9(b) show the two cell structures considered in the simulation: the full cell structure as before, and an identical cell where the back metal is planar rather than structured. The area of the a-Si:H and its structuring are identical between the two cases. From the calculated normalized generation rate plotted in Fig. 7.9(a) we see that many of the spectral features which we attributed to the pitch are in the same wavelength location in both cases, but the amplitude from $\lambda=550$ to $800 \mathrm{~nm}$ is strongly reduced. This supports the idea that metal structuring is essential to achieve high photocurrent enhancements. From $\lambda=350-500 \mathrm{~nm}$ the absorption in the two structures is nearly identical, as expected since the only modification is on the back of the device, and 


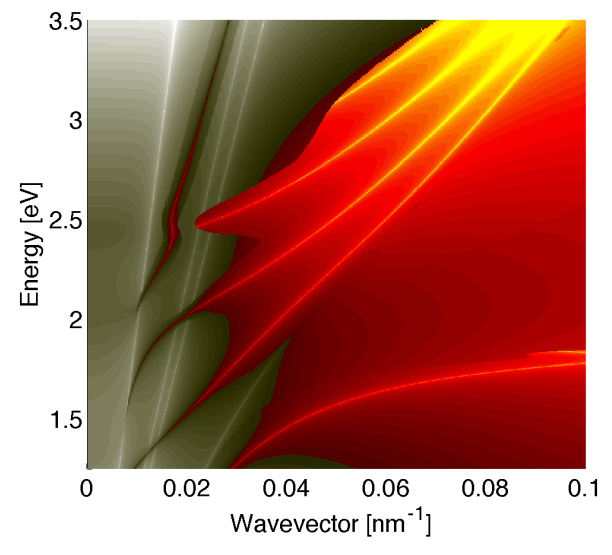

(a) $0 \mathrm{~nm} \mathrm{ZnO}$

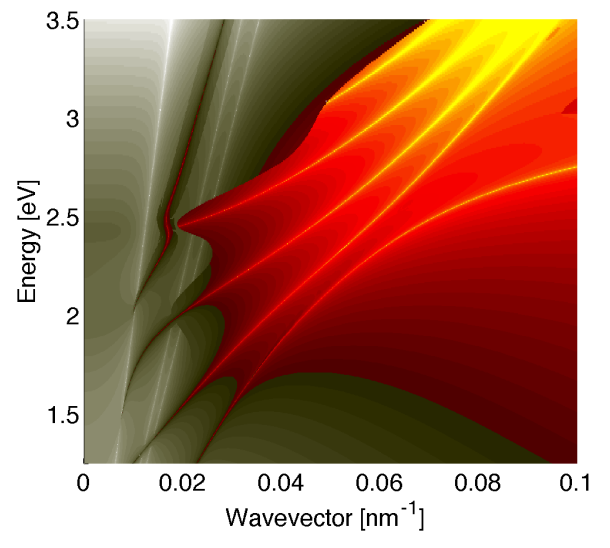

(b) $10 \mathrm{~nm} \mathrm{ZnO}$

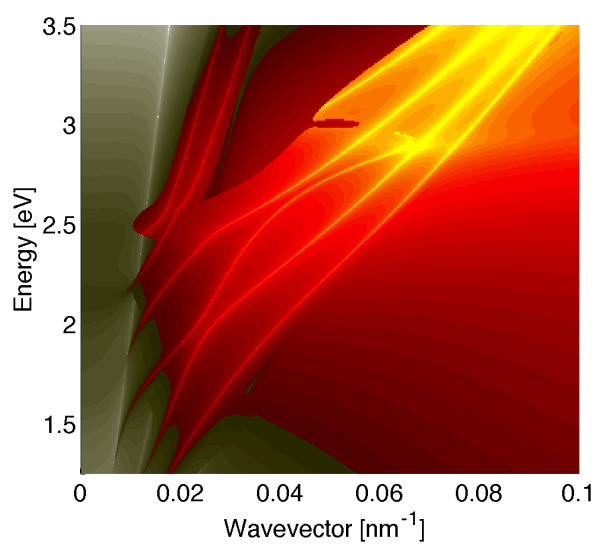

(c) $130 \mathrm{~nm} \mathrm{ZnO}$

Figure 7.8. Dispersion relations for flat layer stack of $\mathrm{Ag} /$ varying ZnO:Al thickness/160 nm a-Si:H/80 nm ITO/air. 


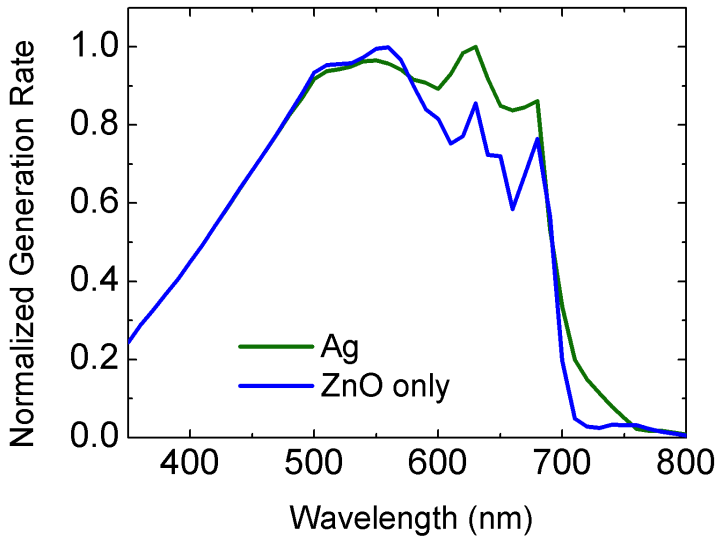

(a) Calculated generation rate.

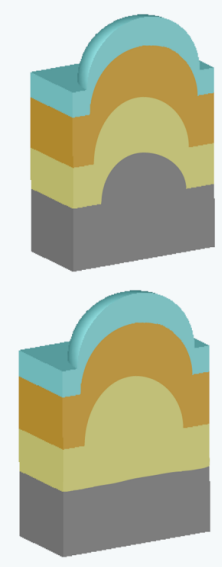

(b) Schematics.

Figure 7.9. Calculated generation rate for $\mathrm{Ag}$ back reflector with and without plasmonic nanostructures.

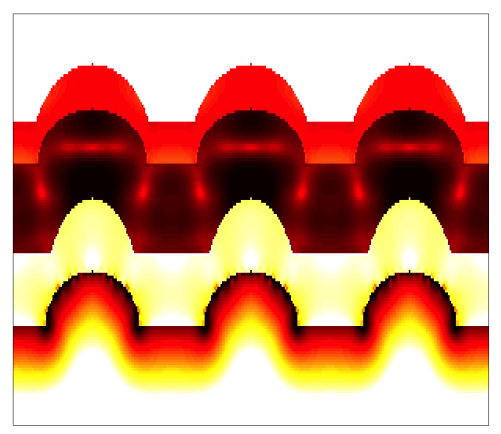

(a) Nanostructured Ag.

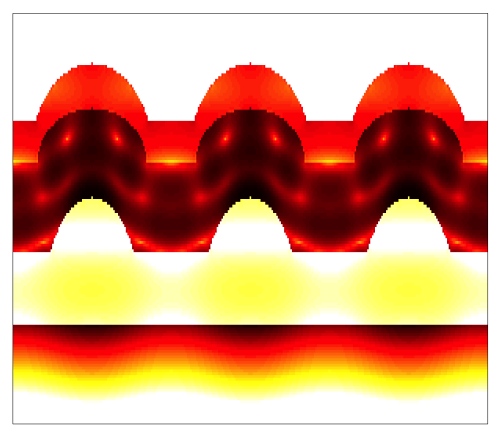

(b) Flat Ag reflector.

Figure 7.10. Photon flux calculated at $\lambda=630 \mathrm{~nm}$.

these wavelengths are absorbed before reaching the back contact. Figure 7.10 shows cross sections of the generation rate for the two cases at $\lambda=630 \mathrm{~nm}$ (on one of the spectral peaks). The modes are clearly different between these two structures, with a particularly high absorption enhancement in the a-Si:H around the scatterer. This example shows the advantage of plasmonic nanostructures over purely dielectric scatterers combined with a back reflector: the presence of the metal does lead to higher photocurrent in the semiconductor, rather than to increased parasitic losses. 


\subsubsection{Other Metals}

As a second way to probe the role of the metal, we investigated the use of other plasmonic metals as back optical materials. In addition to $\mathrm{Ag}$ we modeled $\mathrm{Al}$, which has a plasmonic resonance at shorter wavelengths, and $\mathrm{Au}$ and $\mathrm{Cu}$, which have plasmonic resonances at longer wavelengths than Ag. Figure 7.11 shows the calculated generation rates for identical structures where the back metal material is changed. The structure is the same as discussed above: $300 \mathrm{~nm}$ diameter particles with 500 $\mathrm{nm}$ pitch. As expected, the four metals show identical response from 350 to 500 $\mathrm{nm}$, but the absorption response is quite different from 500 to $700 \mathrm{~nm}$. Interestingly, while many of the spectral features occur at the same wavelengths, the absorption in the a-Si:H when $\mathrm{Al}$ is used as the back contact is much higher than with $\mathrm{Ag}$. We attribute this effect to the shifted plasmon resonance of $\mathrm{Al}$ : at resonance both the scattering cross sections and the absorption cross sections of the particle are high. When this resonance is shifted into the bluer region of the spectrum and the particle is on the back, then the sunlight near the resonance is absorbed before hitting the back contact. In the cases of $\mathrm{Ag}, \mathrm{Au}$, and $\mathrm{Cu}$, however, the resonant wavelengths do interact with the back contact and are parasitically absorbed. $\mathrm{Al}$ is also a known back contact material for a-Si:H with good electrical properties, and is an advantageous choice since it is less expensive and more abundant than Ag.

\subsection{Modeling Random Textures}

\subsubsection{Role of Curvature}

To understand the measured difference in photocurrent between the nanopatterned cells and the randomly textured Asahi cells, we made absorption maps of the photon flux in simulated cells of each type. Figure 7.12 shows cross sections at two different wavelengths, $\lambda=500 \mathrm{~nm}$, and $\lambda=670 \mathrm{~nm}$. It is immediately clear that the periodic nanopatterned cells couple strongly to waveguide modes, whereas the absorption in the randomly textured cells is not clearly connected to mode excitation. A more 


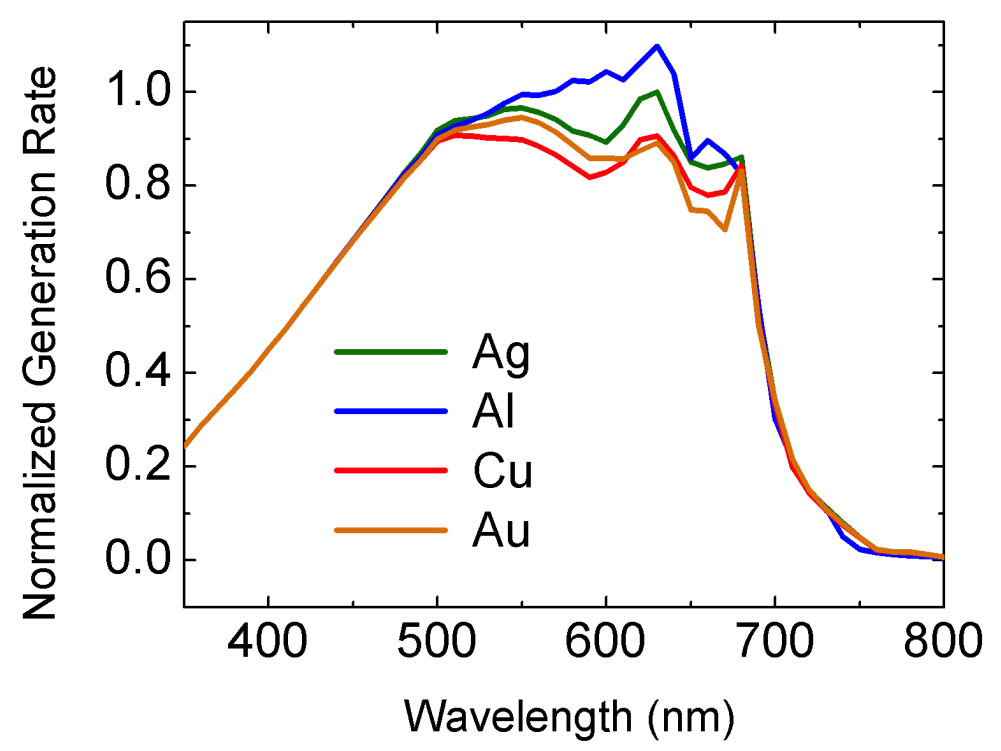

Figure 7.11. Calculated generation rates for identical cells with different back contact materials.

surprising result is the difference in the relative losses in the Ag between the two structures. In the periodic case at $\lambda=500 \mathrm{~nm}$ (Fig. 7.12(a)) dipole-like scattering from the Ag nanoparticles is clearly visible, which translates into higher absorption in the a-Si:H in the region directly above the scatterer and under the top curvature. In the corresponding Asahi case, however, the convex Ag features show very different behavior: these sharper features have higher loss in the Ag, and less absorption in the a-Si:H region directly over them. This figure illustrates the advantage of controlled curvature of the Ag nanopatterns: high frequency components on surfaces and sharp metallic tips are known to lead to high metal absorption, and by rounding the nanoparticles more of the incident light it scattered back into the a-Si:H or coupled to waveguide modes than parasitically absorbed.

\subsection{Optimization of Periodic Nanopatterns}

\subsubsection{Pitch}

The positions of the peaks observed on the red side of the spectrum correspond to the locations of photonic-Bloch modes with high overlap in the a-Si:H layer. Figure 

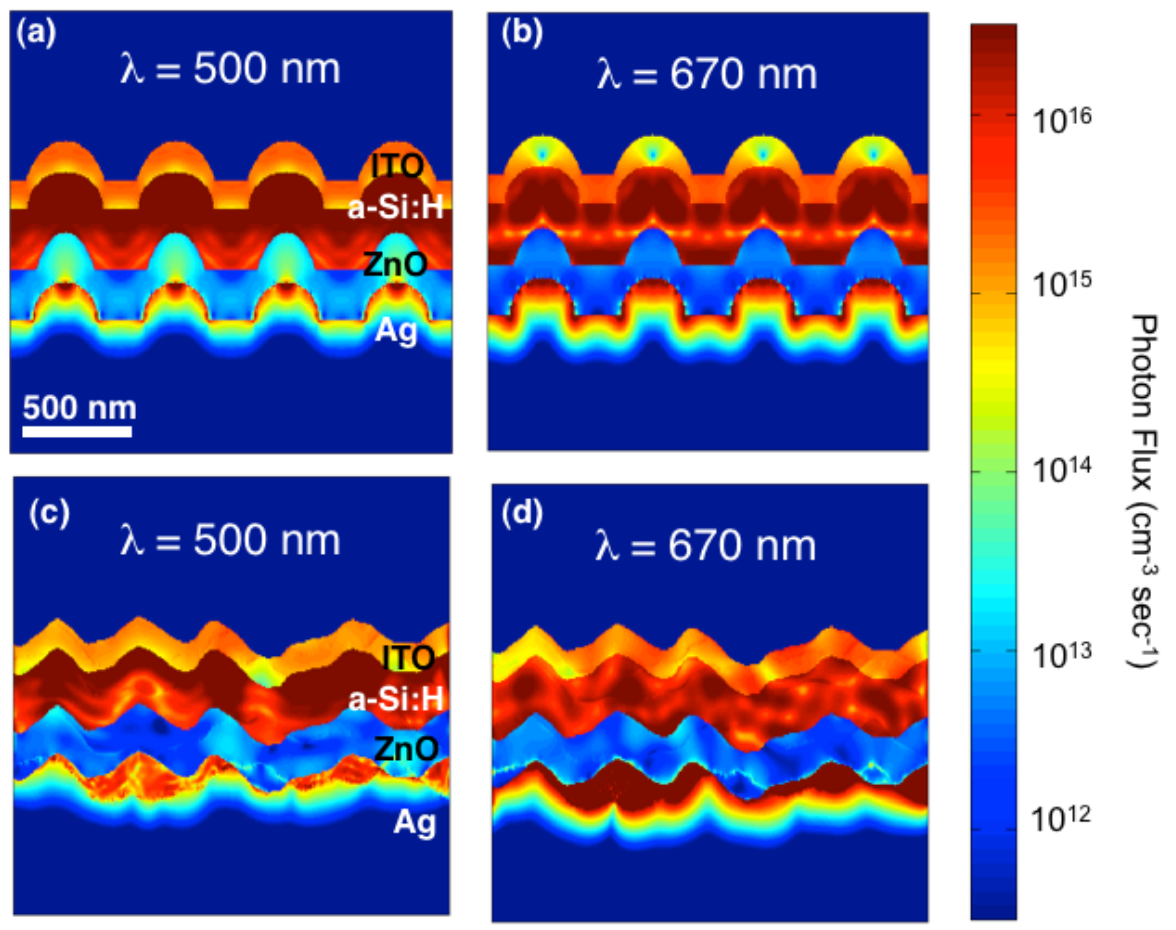

Figure 7.12. Calculated photon flux for cells with $115 \mathrm{~nm}$ thick intrinsic layers. (a)-(b) periodic nanopatterned cells with $500 \mathrm{~nm}$ pitch and $300 \mathrm{~nm}$ diameter Ag. (c)-(d) cells with Asahi U-type texture.

7.13 shows a calculation of how the absorption in the a-Si:H layer is affected by the pitch between the nanostructures. The calculation was done using periodic boundary conditions, and the changing area of the simulation has been corrected to the same volume. Figure 7.13(a) shows a map of the spectral generation rate, with pitch vs. incident wavelength. The color scale is enhancement over the simulated Asahi cell, so that everywhere the map is yellow is enhanced absorption over the Asahi reference, and everywhere that is blue is suppressed. Since the deposition is conformal, the Ag nanopatterns are $300 \mathrm{~nm}$ in diameter but the ITO hemispheres have broadened to $400 \mathrm{~nm}$; the minimum of the pitch axis is thus the closest packed arrangement for a square lattice.

From Fig. 7.13(a) it is evident that the closer pitches of the scatterers are broadly more efficient than the longer spacings. There is a very large, broad region of enhancement extending from 350 to $650 \mathrm{~nm}$ when the spacings are close. Several bands 
139

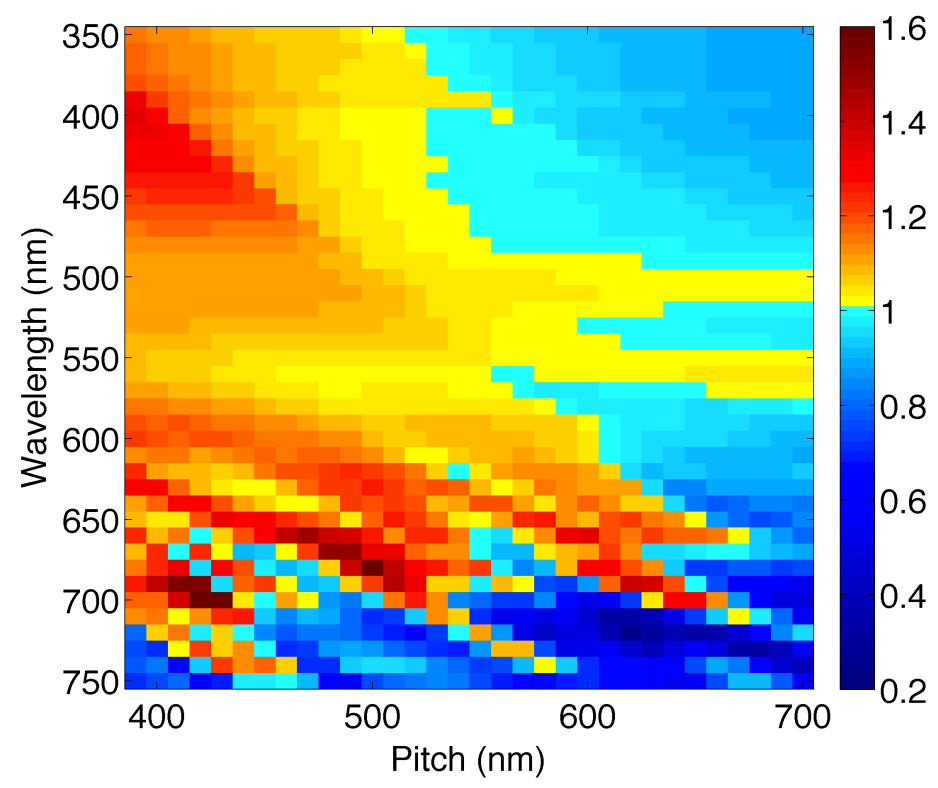

(a) Absorption enhancement map of pitch vs. incident wavelength, where the enhancement is calculated relative to the absorption in a simulated Asahi reference cell.

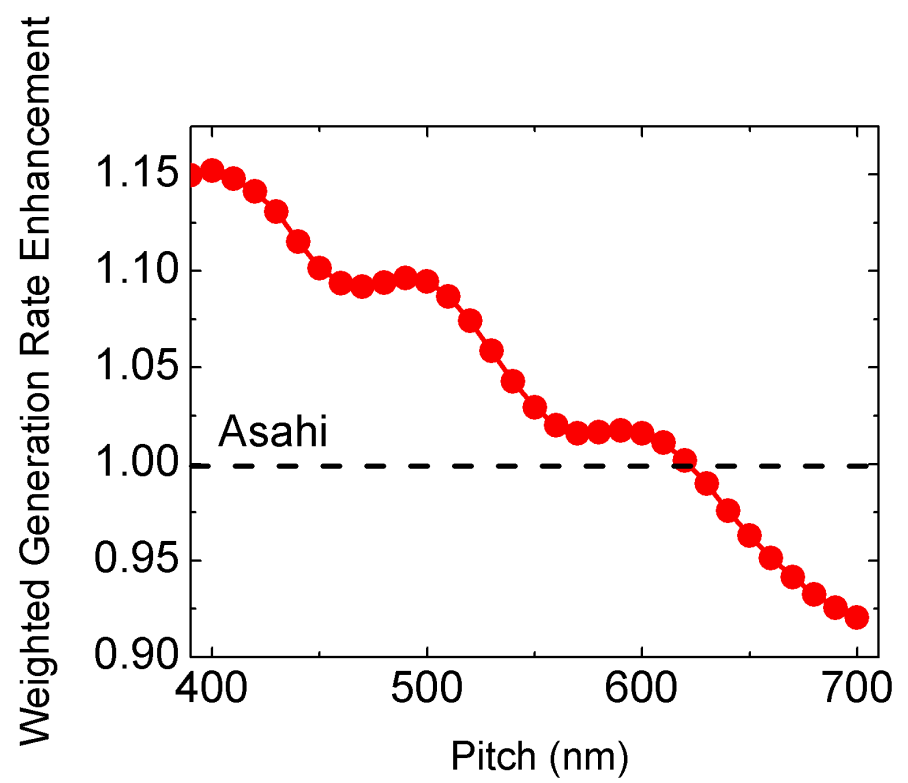

(b) Solar spectrum integrated enhancement in absorption with varying pitch. The Asahi reference cell is shown by the dotted line.

Figure 7.13. Calculated generation rates with varying pitch. The cells have $115 \mathrm{~nm}$ thick intrinsic a-Si:H layers, and the Ag particle is $300 \mathrm{~nm}$ in diameter. 
of high enhancement are visible in the red portion of the spectrum from 650 to 750 $\mathrm{nm}$, where the peak enhancement moves to the blue with shorter pitch. We identify these as the Bloch-photonic modes of the device, and their positions correspond to the peaks shown in Fig. 7.4. There is also a band at very short pitches from $\lambda=$ 400 to $500 \mathrm{~nm}$, where the onset of high absorption corresponds to the $\lambda=$ pitch line. This feature is attributed to the top contact structuring, and will be discussed further in section 8.5. Figure 7.13(b) shows the solar spectrum weighted absorption, calculated by integrating over each vertical position in Fig. 7.13(a). The scale is relative to the simulated Asahi cell, and the arrows depict the pitches that were made experimentally. In the experiment, the device with $500 \mathrm{~nm}$ pitch nanostructures indeed shows about 10\% improvement over the Asahi reference cell, and the $700 \mathrm{~nm}$ pitch cell is markedly suppressed. Although oscillations are present in Fig. 7.13(b), it is notable that the trend is so explicit: shorter pitches lead to higher integrated absorption. At $400 \mathrm{~nm}$ pitch, where the nanostructures are closely packed, there is a peak enhancement value of $15 \%$ over the randomly textured cell.

\subsubsection{Hexagonal Lattice}

Since it is clear from Fig. 7.13(b) that the closest packed position is optimal, hexagonal lattices that allow for closer packing seem like the proper design. Figure 7.14 shows the same calculation of pitch vs. generation rate, for a hexagonal lattice instead of a square lattice. While similar Bloch-photonic modes are seen in Fig. 7.14(a), the enhancement on the red side of the band is much weaker than in the square periodic case. After integrating over the wavelength and weighting by the solar spectrum (Fig. 7.14(b)), we see a completely different response that does not favor the closest packed position, and is frequently below the weighted absorption from the Asahi simulation. In the long wavelength regions, absorption is dominated by coupling to the photonicBloch modes. In the hexagonal lattic, there is one dominant lattice vector, which will lead to one set of photonic-Bloch modes. In the square periodic case, however, there are two, as both the nearest-neighbor and the diagonal scatterers can contribute. For the square lattice, this may lead to broadening of the absorption enhancement on the 


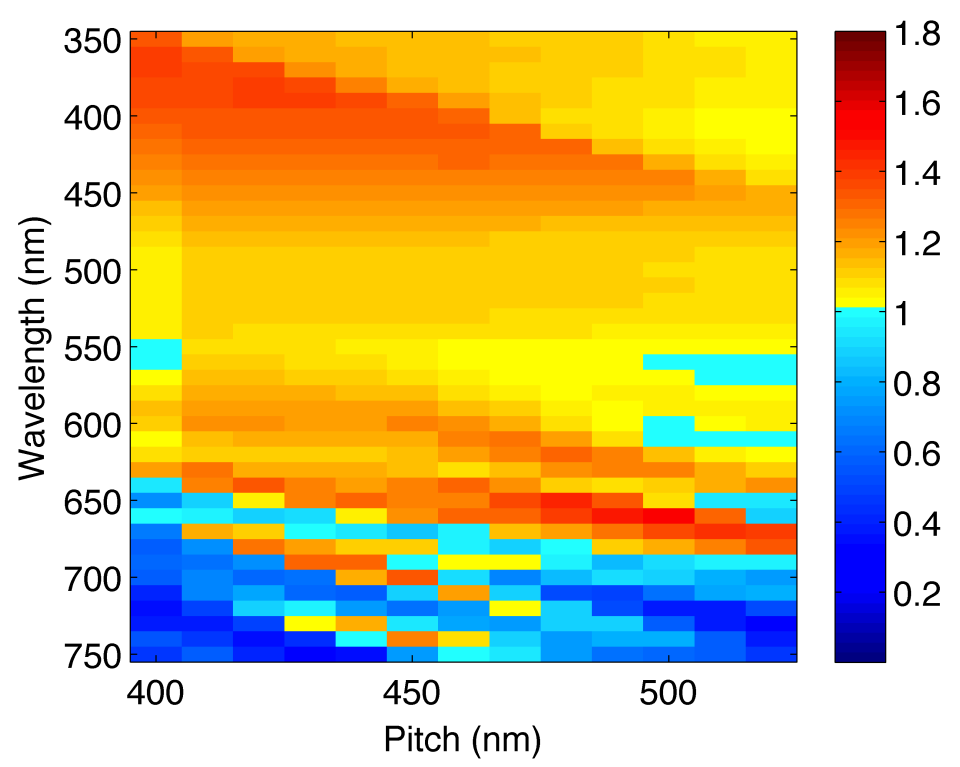

(a) Absorption enhancement map of pitch vs. incident wavelength, where the enhancement is calculated relative to the absorption in a simulated Asahi reference cell.

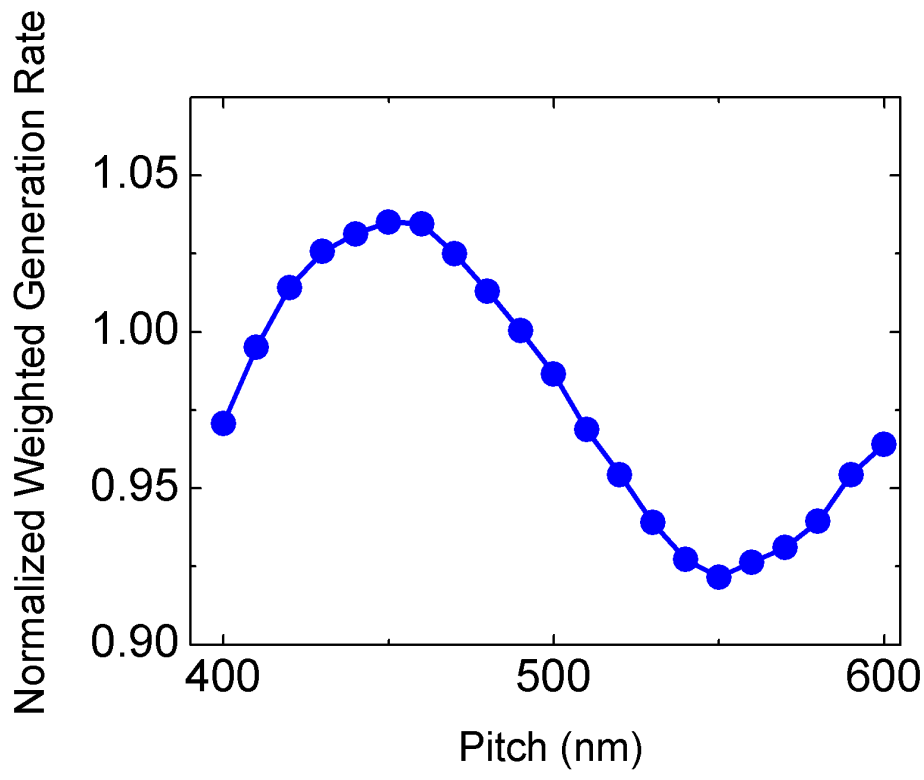

(b) Solar spectrum integrated enhancement in absorption with varying pitch.

Figure 7.14. Calculated generation rates with varying pitch, for a hexagonal lattice. 


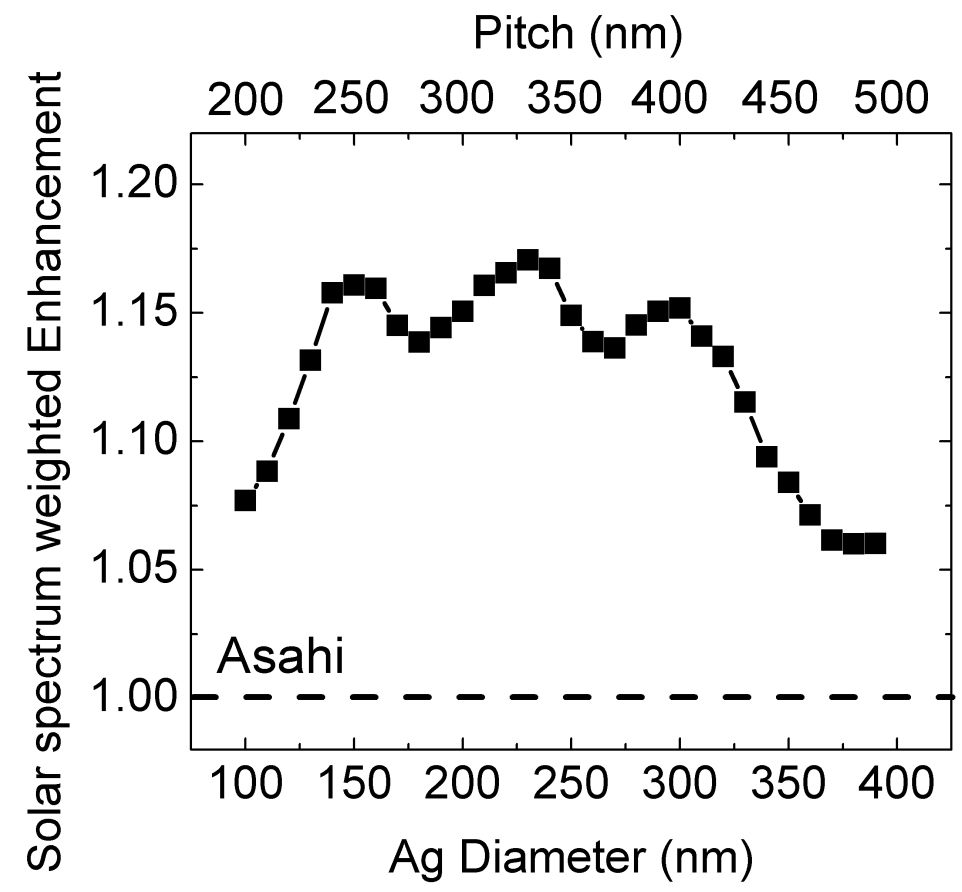

Figure 7.15. Optimization of solar spectrum integrated generation rate with covarying diameter and pitch. Each point is at the closet packed pitch for a given diameter, and the simulated Asahi case is shown as a reference.

red side of the spectrum.

\subsubsection{Pitch and Diameter}

Finally, we combine these studies to predict an optimum geometry and diameter for maximum solar spectrum integrated absorption. Using Fig. 7.13(b) as a guide, we assume that the closest packed square lattice position for any given Ag diameter produces the high absorption. This assumption has been verified by repeating Fig. 7.13(b) for other diameters besides the one shown. In Fig. 7.15 we co-vary the diameter and pitch at the position of maximum packing fraction and plot the enhancement compared to the randomly textured Asahi reference cell. Using Ag as the back contact metal, we predict a maximum absorption enhancement of $17 \%$ at a diameter of $230 \mathrm{~nm}$ and a pitch of $330 \mathrm{~nm}$. Combined with experimentally realizable $\mathrm{V}_{\mathrm{oc}} \mathrm{s}$ and fill factors, this predicts an ultrathin, a-Si:H solar cell with greater than $10 \%$ efficiency. 


\subsection{Conclusions}

Through electromagnetic simulation, we are able to model the light trapping effects in our fabricated solar cells accurately, and use the models to both develop understanding of the physical mechanisms for light trapping across the spectrum and optimize new designs for higher efficiencies. On the blue side of the spectrum, absorption is dominated by the a-Si:H/ITO nanostructures on the front of the cell, and we predict that for closer pitches this will translate to broadband photocurrent enhancement, rather than exclusively red-side photocurrent enhancement. This finding motivates the fabrication of new, optimized solar cells. We also saw the importance of nanoparticle curvature in reducing metal losses relative to randomly textured substrates. The next chapter explores this concept further, constructing pseudo-random solar cells with precisely controlled nanoparticle curvature. And finally, we showed that $\mathrm{Al}$ is a promising back contact material in these designs, with the potential for both low-cost and lower-loss nanostructures. 


\section{Chapter 8}

\section{The Role of Spatial Frequency: High Efficiency a-Si:H Solar Cells}

\subsection{Introduction}

To achieve full absorption of the solar spectrum with ultrathin semiconductor volumes, light trapping is required over a broad spectral and angular range. The previous chapters have shown enhancement of the red side of the spectrum, and predicted arrangements of nanoparticles that lead to additional enhancements on the blue-side of the spectrum. Here we apply those optimized designs to experimental devices, and add an exploration of pseudo-random particle arrangements.

Light trapping nanostructures allow for nanoscale control of the direction and absorption of light in ultrathin-film solar cells, enabling new designs in which light is absorbed in semiconductor volumes much thinner than the optical absorption length. Indeed, an ultrathin- film solar cell can be considered as an optical integrated circuit in which light is received, guided, localized and collected at the nanoscale. Here we integrate both plasmonic metal nanostructures and semiconductor Mie resonators into the solar cell to trap light into both in-plane waveguide modes and localized modes.

To date, light trapping geometries based on nanowires, nanocones, photonic crystals, nanoparticles, gratings, and random textures have been demonstrated [18, 19, $20,21,22,23,24,25,26,27,28,29,116,117,131,135,136]$. While many researchers have demonstrated increased photocurrent due to scattering-mediated light trapping, the role of spatial correlations and surface topography of random or periodic arrange- 
ments of the scattering nanostructures has remained unclear. We identify the relation between these key parameters and the photocurrent spectrum for high efficiency (> 9.5\%) ultrathin-film ( $<100 \mathrm{~nm}$ thick absorber) a-Si:H solar cells containing both integrated plasmonic and Mie scattering nanostructures. The enhanced performance of the nanostructure arrays described here signals a new direction for enhanced absorption in which light trapping nanopatterns, defined by their spatial coherence spectral density, are designed for broadband and isotropic response.

Most light trapping geometries studied, particularly for a-Si:H, are based on random scattering structures with the geometry determined by the random nature of a deposition, growth, or etch process rather than by design. This is due to the fact that no suitable techniques were available that enable the controlled fabrication of engineered random scattering arrays at low cost. Recently, soft imprint lithography has been introduced as a technique that enables large-area, inexpensive fabrication of arrays of nanoscatterers $[124,129,137,138]$. We have shown that periodic arrays made using this technique show enhanced photocurrents in ultrathin-film a-Si:H solar cells over a narrow spectral range [24]. This new technique now also enables the design and fabrication of engineered random arrays, with the spatial frequencies in the array pattern optimized for trapping a broad solar spectral band that is otherwise poorly absorbed.

In this chapter, we systematically design and fabricate periodic and random arrays of nanoscatterers integrated in the back contact of an a-Si:H solar cell. By tailoring the spatial frequencies and obtaining an integrated understanding of both the localized and propagating modes resulting from plasmonic and Mie scattering in the three-dimensional structure, we are able to demonstrate an extremely thin cell with broadband photocurrent enhancement in both the blue and the red portions of the spectrum. 


\subsection{Design and Fabrication of Engineered Random Arrays}

To test these new patterns, we fabricated a new stamp similar to that discussed in section 6.6. Figure 8.1 shows most of the patterns used in the experiment and their Fourier transforms. While AFM characterization was done for each pattern over four different scan sizes, the $5 \mu \mathrm{m}$ x $5 \mu \mathrm{m}$ scans are shown in Fig. 8.1. The nanoparticle sizes and shape are seen to be uniform across the substrate. All of the nanoparticle patterns (patterns 1-11) are $100 \mathrm{~nm}$ tall. Three diameters of nanoparticles were used in separate patterns, denoted small, medium, and large, corresponding to $200 \mathrm{~nm}$, $290 \mathrm{~nm}$, and $310 \mathrm{~nm}$ diameter, respectively, after overcoating. (The master wafer had diameters of $75 \mathrm{~nm}, 125 \mathrm{~nm}$, and $175 \mathrm{~nm}$.) The first twelve were arranged in two rows of 6 on the master substrate, then repeated with rotation to control for deposition inhomogeneities. A thirteenth pattern, of Asahi U-type texture, was included. In summary, the thirteen nanopatterns used were:

1. Periodic, $400 \mathrm{~nm}$ pitch, $200 \mathrm{~nm}$ Ag particles (a)

2. Periodic, $400 \mathrm{~nm}$ pitch, $290 \mathrm{~nm}$ Ag particles (b)

3. Periodic, $400 \mathrm{~nm}$ pitch, $310 \mathrm{~nm} \mathrm{Ag} \mathrm{particles} \mathrm{(c)}$

4. Periodic, $500 \mathrm{~nm}$ pitch, $200 \mathrm{~nm}$ Ag particles

5. Periodic, $500 \mathrm{~nm}$ pitch, $290 \mathrm{~nm}$ Ag particles

6. Periodic, $500 \mathrm{~nm}$ pitch, $310 \mathrm{~nm}$ Ag particles

7. Pseudo-random, $200 \mathrm{~nm}$ particles (d)

8. Pseudo-random, $290 \mathrm{~nm}$ particles (e)

9. Pseudo-random, $310 \mathrm{~nm}$ particles (f)

10. Pseudo-random, varying size (mixture of $200 \mathrm{~nm}, 290 \mathrm{~nm}$, and $310 \mathrm{~nm}$ particles)

11. Penrose, $290 \mathrm{~nm}$ particles (h)

12. Flat

13. Asahi U-type texture (i) 


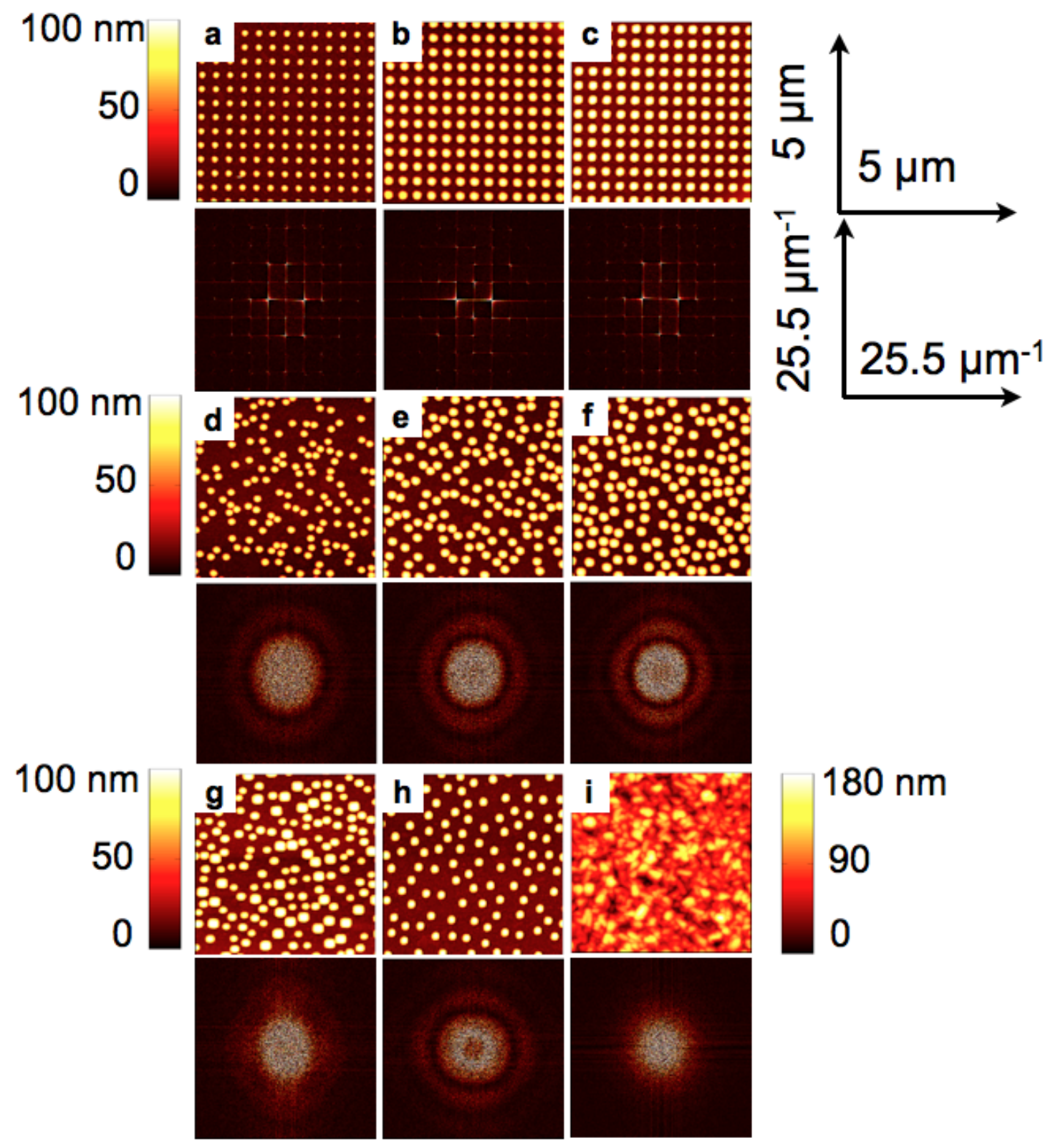

Figure 8.1. AFM images and fourier transforms of the Ag-coated patterns used in the experiments. (a)-(c) $400 \mathrm{~nm}$ periodic lattice with $200 \mathrm{~nm}, 290 \mathrm{~nm}$, and $310 \mathrm{~nm}$ nanoparticles, respectively. (d)-(f) Pseudo-random lattices with $200 \mathrm{~nm}, 290 \mathrm{~nm}$, and $310 \mathrm{~nm}$ nanoparticles. (g) Pseudo-random lattice with varying particle size. (h) Penrose pattern of $290 \mathrm{~nm}$ particles. (i) Replica Asahi U-type texture. 
The $400 \mathrm{~nm}$ pitch periodic lattice was chosen based on electromagnetic simulation. Figure 7.13 shows the wavelength-dependent absorption enhancement vs. pitch. Integrating the wavelength-dependent calculated absorption by the AM1.5 solar spectrum produces a figure of merit similar to a short circuit current density. Fig. 7.13(a) shows the result of weighting Fig. 7.13(b) by the solar spectrum. The cells in this case have $115 \mathrm{~nm}$ intrinsic layers, with a $300 \mathrm{~nm} \mathrm{Ag}$ particle, $325 \mathrm{~nm} \mathrm{ZnO}$ :Al particle, $375 \mathrm{~nm}$ a-Si:H particle, and $400 \mathrm{~nm}$ ITO particle. While there are visible oscillations due to the presence of the waveguide modes, the outstanding trend is that packing fraction is critical: the closest packed position for these diameters is the optimal position. This trend was verified with other diameters. The size of the experimental large Ag pillars was chosen so that the resulting ITO overcoated patterns would be close-packed.

For the pseudo-random patterns, each pattern was uniquely generated with the constraints that the Ag particles do not touch after overcoating, and that the packing fraction is the same as for the $400 \mathrm{~nm}$ periodic pattern. The center points of each pattern were generated over a $100 \mu \mathrm{m}$ x $100 \mu \mathrm{m}$ area, and tiled to form a $6 \mathrm{~mm} \mathrm{x}$ $6 \mathrm{~mm}$ array with careful treatment of the edges to avoid overlapping nanoparticles. The penrose pattern has a different packing fraction than the other patterns, but the minimum distance between particles was set to $400 \mathrm{~nm}$, the same as in the periodic pattern.

\subsubsection{Calculation of Power Spectral Density}

When the absorption length in a-Si:H is longer than the separation between particles (for $\lambda=600 \mathrm{~nm}$ ) the arrangement of the nanoparticles becomes more important and the incident sunlight will couple to propagating modes of the device. In the periodic case coupling only occurs for wavelengths where the pattern bridges the wavevector mismatch between incoming light and waveguide modes, leading to peaks in the photocurrent. Truly random surface textures should show a broadband response, as the spatial frequencies present in the surface should be evenly distributed. 


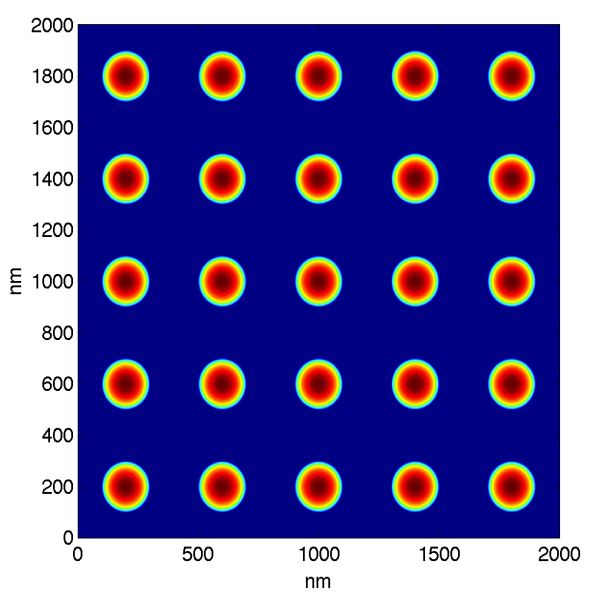

(a) $400 \mathrm{~nm}$ pitch periodic lattice

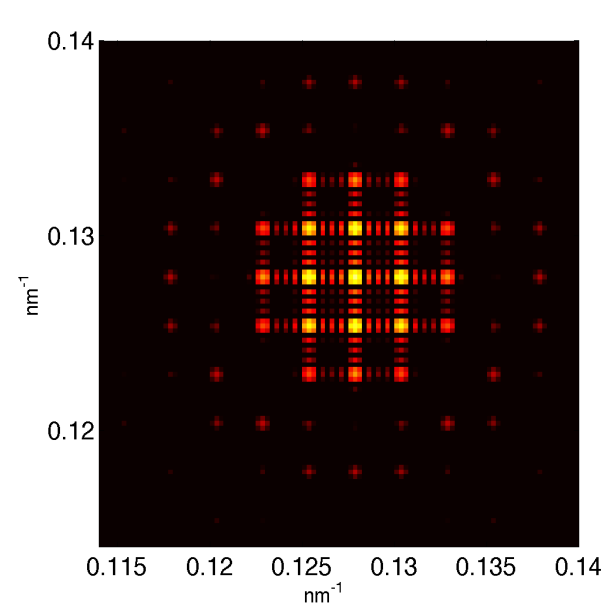

(c) Power spectrum

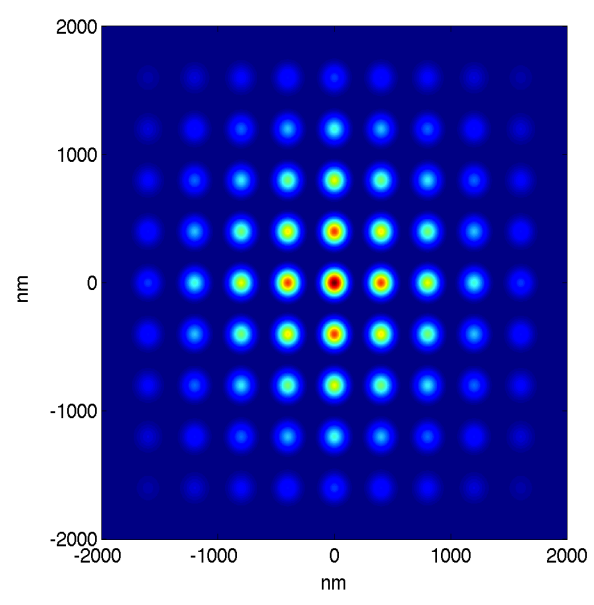

(b) Autocorrelation of periodic lattice

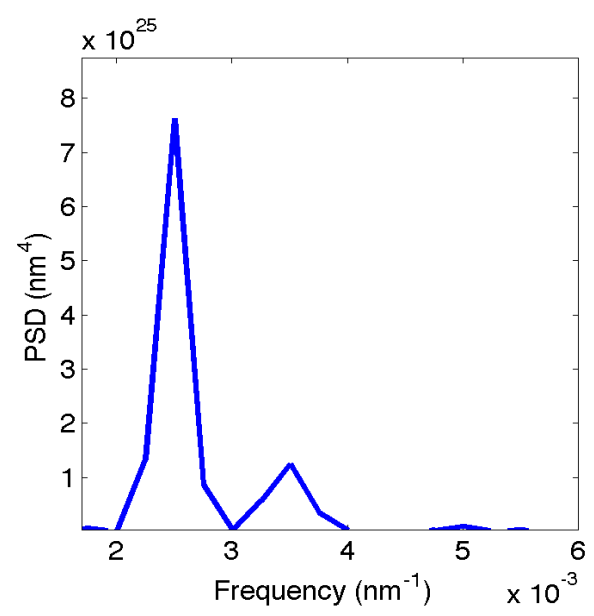

(d) Power spectral density

Figure 8.2. Calculation of two-dimensional power spectral density from surface topography. 


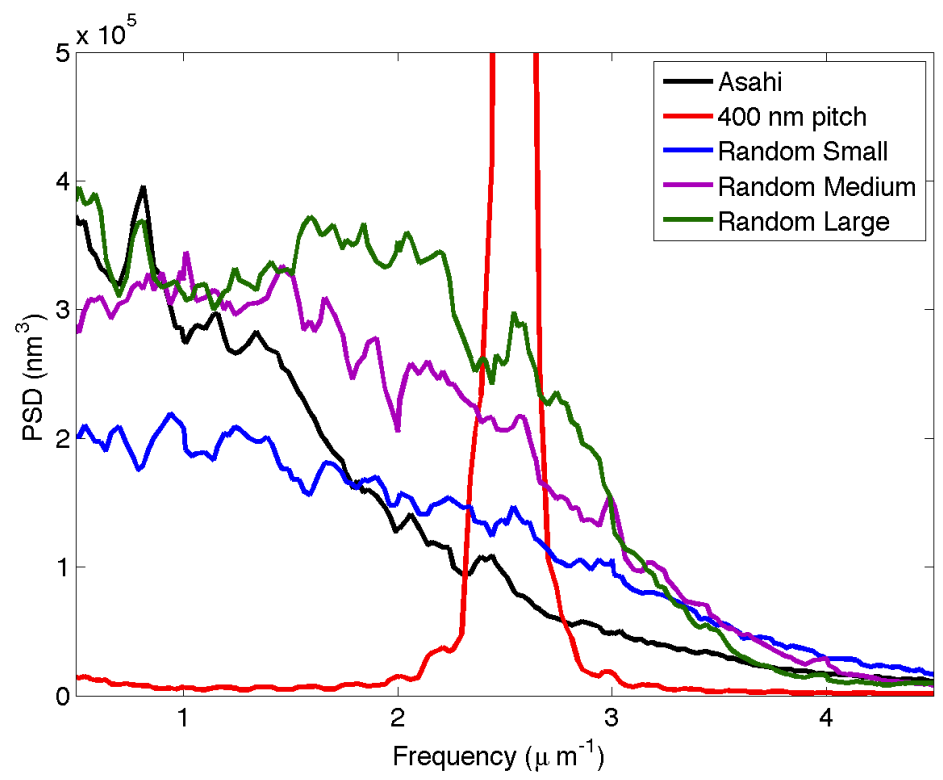

Figure 8.3. Power spectral density calculated from AFM data of Ag-coated surfaces.

For the purposes of light trapping, we thus define "random" by the power spectral density (PSD) of the surface: a random surface is one that possesses all spatial frequencies in equal amounts. Of course a flat surface will also meet this criterion, so the ideal surface must also possess a high PSD. Our aim is to design a nanopattern with a sufficient distribution of spatial frequencies to be random, but only over the range of spatial frequencies that can excite modes in the semiconductor. At all other spatial frequencies the power spectrum should be at a minimum. While this is useful for plasmonic photovoltaic design, the principle is general and can apply to other types of light trapping surfaces.

Figure 8.2 outlines the calculation procedure for determining the PSD of a given surface. We start with a surface topography, as obtained from AFM measurement or designed in Matlab (Fig. 8.2(a)). In this case it is a $400 \mathrm{~nm}$ pitch square periodic pattern of hemispheres. We then calculate the two-dimensional autocorrelation of the surface, shown in Fig. 8.2(b). From the autocorrelation in this case it is clear that the dominant pitch between all particles is $400 \mathrm{~nm}$. We then take the fourier transform of the autocorrelation, and calculate the power spectrum of the surface (Fig. 8.2(c)). Radially integrating the power spectrum gives the PSD (Fig. 8.2(d)). 
Figure 8.3 shows the PSD [139] of the patterned back contacts from the AFM data shown in Fig. 8.1. For experimental data sets obtained from AFM data, it is preferable to use one-dimensional PSD calculations across the direction of the AFM scan, to retain information about tip artifacts. The periodic, $400 \mathrm{~nm}$ pitch pattern, unsurprisingly, has a very sharp band centered at $2.5 \mathrm{\mu m}^{-1}$, with a bandwidth due to the finite size of the AFM scan. The Asahi and pseudo-random nanopatterns are much broader and flatter in spatial frequency. Notably, the designed pseudo-random pattern with large particles has a higher PSD across the range of spatial frequencies approximately matching the wavelengths that a- $\mathrm{Si}: \mathrm{H}$ is active.

\subsubsection{Fabrication of Replica Asahi Substrates}

In previous work, we used Asahi U-type glass (textured $\mathrm{SnO}_{2}$ F on glass) as a reference sample. For this work, we sought to replicate the Asahi U-type texture into the same silica sol-gel resist used in the fabrication of our nanopatterned samples. Using the same substrate materials before Ag deposition is a more thorough control experiment, as there should not be significant differences in the Ag coating process.

As noted by others but not explored here, the ability to replicate random textures of one material into any second material is broadly applicable. Potentially, any material with interesting native texture could be transferred into any second material with preferable optical properties. For example, texture from one transparent conducting oxide can be transferred into a lower loss TCO for a p-i-n solar cell [138].

Similarly to the process used in molding stamps from the master Si wafer patterned by electron beam lithography, a composite PDMS stamp was molded from the original texture, and embossed into silica sol-gel on glass. Figure 8.4 shows AFM comparisons of original Asahi U-type glass and replica Asahi U-type texture in silica sol-gel, which look very similar. To quantify the comparison, Fig. 8.5 shows a comparison of the power spectral density from the original and replica samples. AFM scans were taken on each substrate over three different $2 \mu \mathrm{m} \times 2 \mu \mathrm{m}$ scan areas and one $5 \mu \mathrm{m} \times 5 \mu \mathrm{m}$, then calculated in one-dimensional for the reasons discussed earlier. The four traces of each color shown on the PSD diagram show the results of the PSD calculation for 


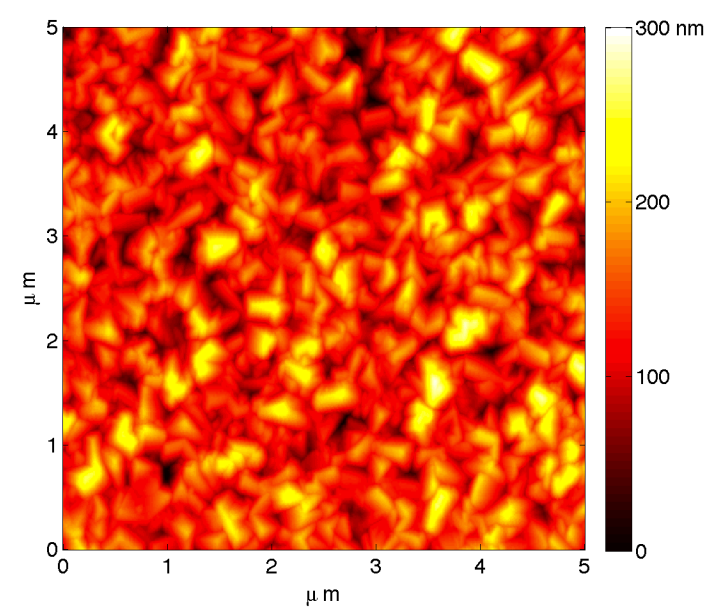

(a) Original

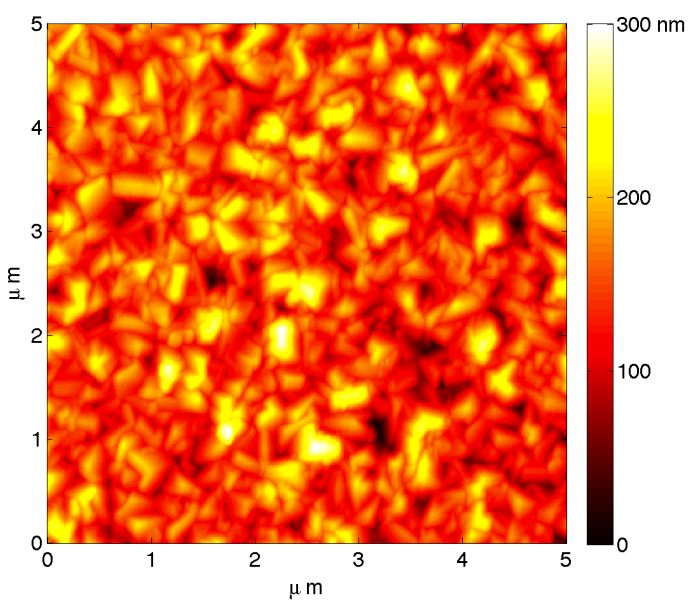

(b) Replica

Figure 8.4. AFM comparison of original Asahi U-type texture and replica texture in silica sol-gel.

each scan area. The original and the replica agree closely, which confirms that the replica Asahi texture is an appropriate reference layer.

Figure 8.6 shows the results of external quantum efficiency measurements on cells with $115 \mathrm{~nm}$ intrinsic layer thickness grown in the same deposition run over the two Ag-coated substrates. The spectra plotted are the average external quantum efficiency over several different cells on each substrate. Remarkably, the EQE measurements show slightly better photocurrent response for the replica substrate over the original substrate. The initial sputter coating conditions of Ag over the Asahi original glass $\left(\mathrm{SnO}_{2:} \mathrm{F}\right)$ and the silica sol-gel will differ slightly, which could affect the resulting surface roughness slightly.

\subsection{Solar Cell Measurements}

\subsubsection{Characterization of Topography}

Cells were deposited over identical substrates with three different intrinsic layer thicknesses: $90 \mathrm{~nm}, 115 \mathrm{~nm}$, and $150 \mathrm{~nm}$. The cells consist of $100 \mathrm{~nm}$ of Ag coating the patterned silica sol-gel, $130 \mathrm{~nm}$ of $\mathrm{ZnO}: \mathrm{Al}$, n-i-p a-Si:H of varying i-layer thickness, 


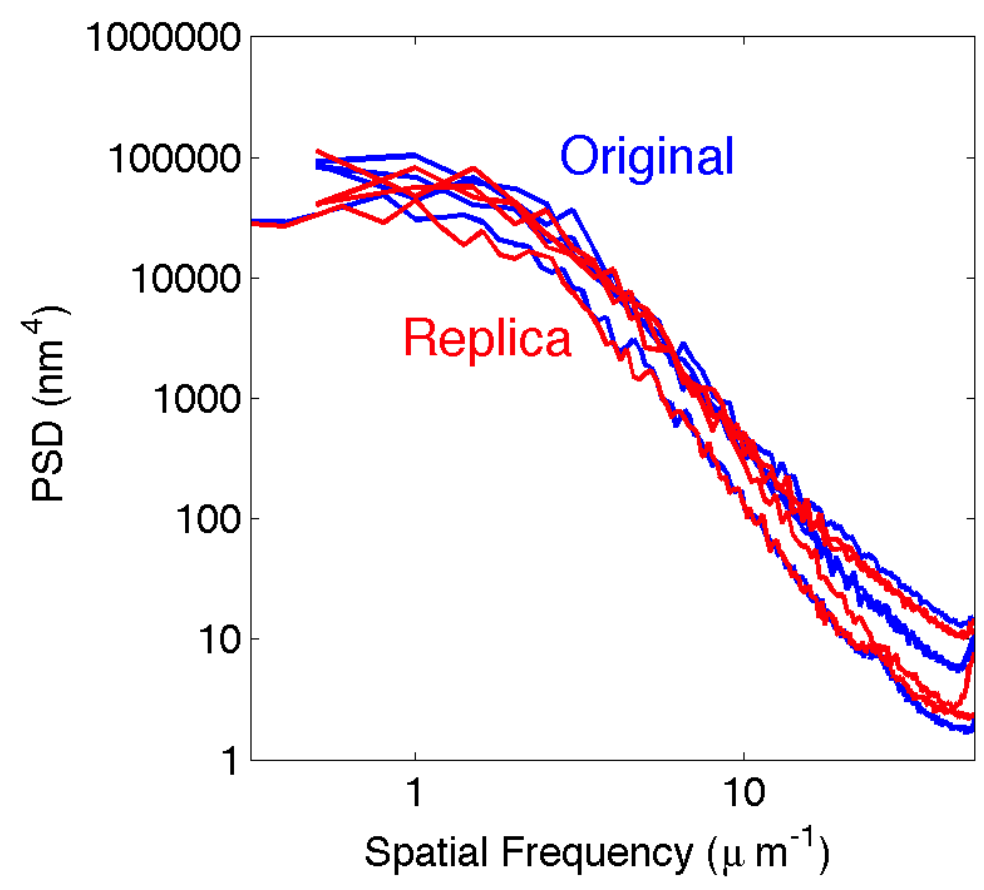

Figure 8.5. Power spectral density calculations comparing the original Asahi U-type texture and replica texture in silica sol-gel.

$80 \mathrm{~nm}$ of ITO, and evaporated Au finger contacts. Figure 8.7 shows cross sections through a few cells illustrating the conformal nature of the growth. Device cross sections were made by a $\mathrm{CO}_{2}$ laser along the backside of the cells, which lead to controlled splitting of the samples allowing for clean cross sections. The cut samples were then imaged with scanning electron microscopy. Figure 8.7(a) shows a close-up view of one of the $90 \mathrm{~nm}$ thick cells fabricated on a periodic array of Ag particles, which confirms that the deposited layer is approximately $90 \mathrm{~nm}$ thick assuming $45 \mathrm{~nm}$ of doped layers. Figures 8.7(b), 8.7(c), and 8.7(d) show cross sections of cells made on the periodic, replica Asahi, and pseudo-random textures, such that the propagation of texture from the back to front of the device is clearly visible. Figure 8.8 shows an AFM scan made on the top interface of a fully fabricated solar cell, indicating the fidelity and regularity of the transferred patterns. 
155

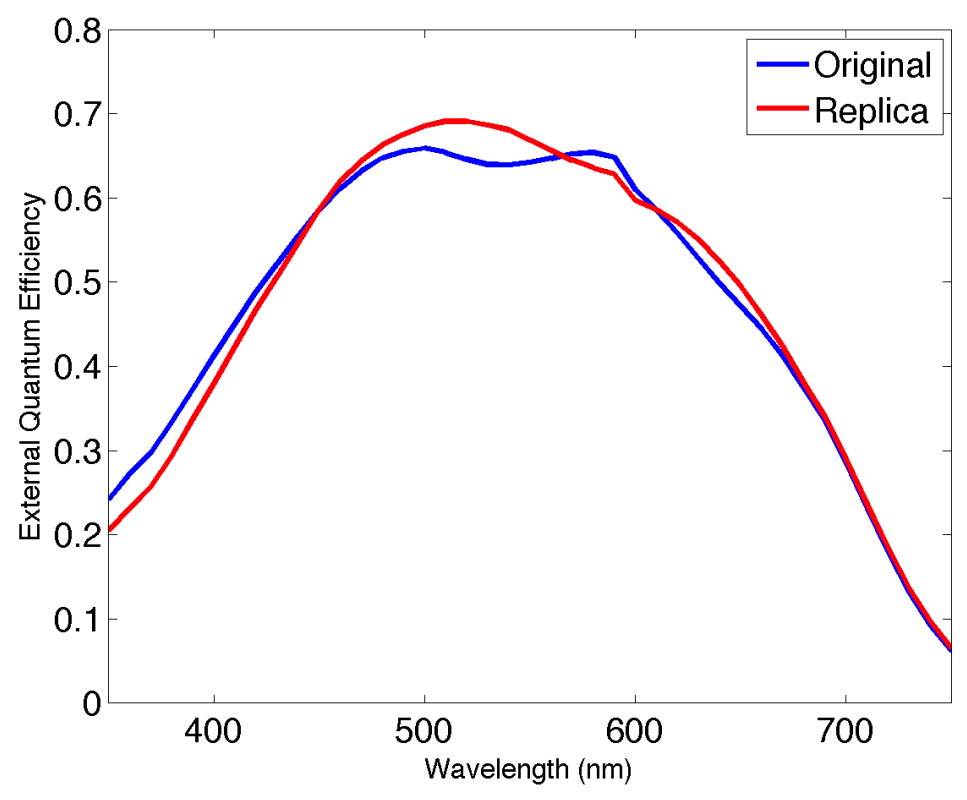

Figure 8.6. EQE measurements of cells with $115 \mathrm{~nm}$ thick i-layers grown on Ag coated Asahi U-type glass (original) and replicated Asahi U-type texture in silica sol-gel (replica).

\subsubsection{J-V Measurements}

The solar cells were characterized under AM1.5 conditions and standard solar illumination of $100 \mathrm{~mW} \mathrm{~cm}{ }^{-2}$. Figure 8.9 shows current-voltage measurements on the cells with $90 \mathrm{~nm}$ intrinsic layers for some selected, high-performing cell patterns. Summaries of the results for each thickness are shown in Tables 8.1, 8.2, and 8.3. While several copies of each cell pattern were made, the best efficiency values are tabulated here rather than the average. Some of the cells on the substrate are shunted, so it is more appropriate to compare cells located near each other on the substrate instead of averaging over an unequal number of working devices. Where patterns are missing, all of the cells of that pattern on the substrate were shunted. Over 1000 cells were measured in total for this study.

Several important trends are observed from comparison of the nanopatterns. The cells of $400 \mathrm{~nm}$ pitch have higher $\mathrm{J}_{\mathrm{sc}}$ than those with $500 \mathrm{~nm}$ pitch. Cells with large and medium diameter nanoparticles show higher currents than those with small nanoparticles. The highest efficiency $(9.4 \%-9.6 \%)$ for the thinnest layers is observed 


\begin{tabular}{|c|c|c|c|c|c|}
\hline Thickness $(\mathrm{nm})$ & Pattern & Eff. $(\%)$ & $\mathrm{J}_{\mathrm{sc}}\left(\mathrm{mA} / \mathrm{cm}^{2}\right)$ & $\mathrm{V}_{\mathrm{oc}}(\mathrm{V})$ & $\mathrm{FF}$ \\
\hline 90 & $400 /$ Large & 9.35 & 16.24 & 0.920 & 0.63 \\
90 & $400 /$ Medium & 9.60 & 16.94 & 0.864 & 0.66 \\
90 & $400 /$ Small & 8.87 & 16.10 & 0.865 & 0.64 \\
90 & $500 /$ Large & 8.72 & 15.85 & 0.870 & 0.63 \\
90 & $500 /$ Medium & 8.77 & 15.37 & 0.889 & 0.64 \\
90 & $500 /$ Small & 8.66 & 14.60 & 0.897 & 0.66 \\
90 & Random/Large & 9.44 & 16.84 & 0.872 & 0.64 \\
90 & Random/Medium & 9.35 & 16.18 & 0.888 & 0.65 \\
90 & Random/Small & 7.27 & 14.22 & 0.835 & 0.61 \\
90 & Random/Varying & 8.88 & 16.68 & 0.855 & 0.62 \\
90 & Penrose & 9.26 & 16.72 & 0.864 & 0.64 \\
90 & Flat & 6.32 & 11.52 & 0.845 & 0.65 \\
90 & Asahi & 6.94 & 12.52 & 0.890 & 0.62 \\
\hline
\end{tabular}

Table 8.1. JV measurements on cells with $90 \mathrm{~nm}$ intrinsic layer thickness 


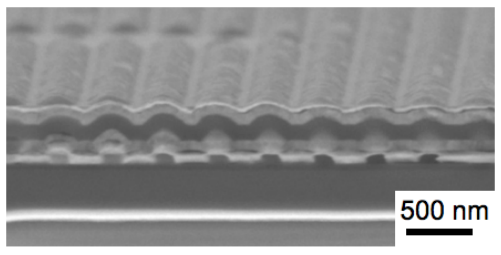

(a) Periodic

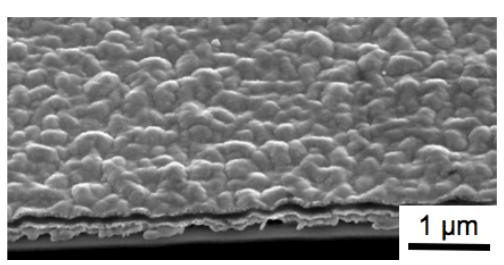

(c) Asahi

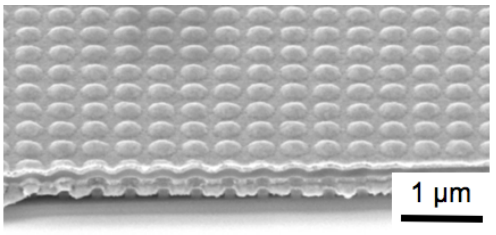

(b) Periodic

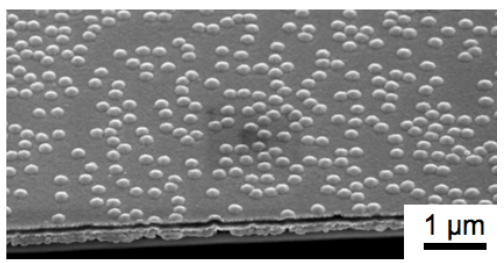

(d) Pseudo-Random

Figure 8.7. SEM cross sections of solar cells deposited over various light trapping patterns. The conformal nature of the depositions is clearly visible, and the patterns are transferred through to the top ITO layer with good fidelity.

for the $400 \mathrm{~nm}$ pitch pattern with medium particles and the random pattern with large particles. These two patterns consistently perform well at other intrinsic layer thicknesses. These efficiencies are among the highest measured for nanostructured a-Si:H solar cells, and are especially notable given the very small (90 $\mathrm{nm}$ ) intrinsic layer thickness.

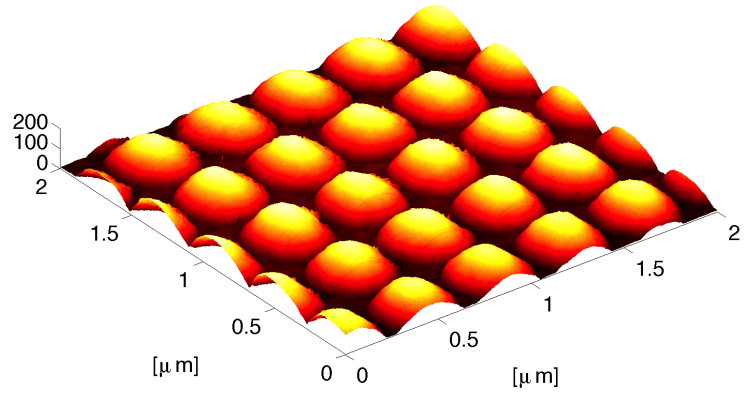

Figure 8.8. AFM scan of ITO top surface of cells deposited over 400 nm pitch periodic patterns. 


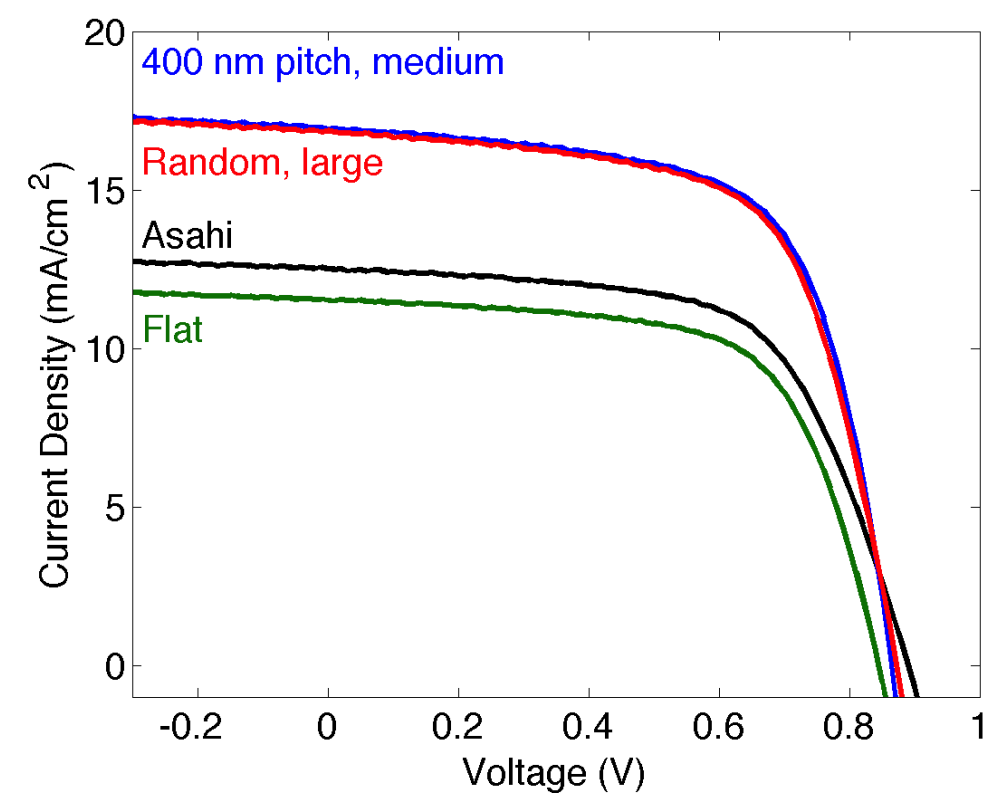

Figure 8.9. Current-voltage measurements for highly performing cells with $90 \mathrm{~nm}$ thick intrinsic layers.

\begin{tabular}{|c|c|c|c|c|c|}
\hline Thickness $(\mathrm{nm})$ & Pattern & Eff. $(\%)$ & $\mathrm{J}_{\mathrm{sc}}\left(\mathrm{mA} / \mathrm{cm}^{2}\right)$ & $\mathrm{V}_{\text {oc }}(\mathrm{V})$ & $\mathrm{FF}$ \\
\hline 115 & $400 /$ Large & 9.37 & 15.71 & 0.912 & 0.65 \\
115 & $400 /$ Small & 5.91 & 13.66 & 0.833 & 0.52 \\
115 & 500/Large & 7.54 & 14.31 & 0.862 & 0.61 \\
115 & $500 /$ Medium & 5.88 & 12.92 & 0.789 & 0.58 \\
115 & $500 /$ Small & 5.75 & 12.26 & 0.782 & 0.60 \\
115 & Random/Large & 7.49 & 14.93 & 0.834 & 0.60 \\
115 & Random/Medium & 6.47 & 13.53 & 0.787 & 0.61 \\
115 & Random/Small & 6.23 & 10.95 & 0.890 & 0.63 \\
115 & Random/Varying & 8.06 & 15.90 & 0.832 & 0.61 \\
115 & Penrose & 7.64 & 15.57 & 0.812 & 0.60 \\
115 & Flat & 3.61 & 9.770 & 0.737 & 0.50 \\
\hline
\end{tabular}

Table 8.2. JV measurements on cells with $115 \mathrm{~nm}$ intrinsic layer thickness 


\begin{tabular}{|c|c|c|c|c|c|}
\hline Thickness $(\mathrm{nm})$ & Pattern & Eff. $(\%)$ & $\mathrm{J}_{\mathrm{sc}}\left(\mathrm{mA} / \mathrm{cm}^{2}\right)$ & $\mathrm{V}_{\text {oc }}(\mathrm{V})$ & $\mathrm{FF}$ \\
\hline 150 & $400 /$ Large & 7.38 & 14.94 & 0.755 & 0.65 \\
150 & $400 /$ Medium & 9.48 & 16.57 & 0.854 & 0.67 \\
150 & $400 /$ Small & 8.26 & 15.54 & 0.818 & 0.65 \\
150 & $500 /$ Large & 8.44 & 15.54 & 0.829 & 0.66 \\
150 & $500 /$ Medium & 7.99 & 16.22 & 0.797 & 0.62 \\
150 & $500 /$ Small & 6.55 & 13.95 & 0.733 & 0.64 \\
150 & Random/Large & 8.17 & 16.53 & 0.797 & 0.62 \\
150 & Random/Medium & 7.42 & 15.99 & 0.787 & 0.59 \\
150 & Random/Small & 6.90 & 14.68 & 0.764 & 0.61 \\
150 & Random/Varying & 8.02 & 15.47 & 0.809 & 0.64 \\
150 & Penrose & 7.44 & 15.69 & 0.783 & 0.60 \\
150 & Asahi & 7.61 & 14.13 & 0.808 & 0.67 \\
\hline
\end{tabular}

Table 8.3. JV measurements on cells with $150 \mathrm{~nm}$ intrinsic layer thickness 


\subsubsection{EQE Measurements}

External quantum efficiency measurements for representative cells of each intrinsic layer thickness are shown in Fig. 8.10. All of the textured and nanopatterned devices show enhanced photocurrent on the red side of the spectrum, and the periodic nanopatterns additionally show several sharp peaks that are correlated with pitch. We have previously shown these features are due to waveguide modes with high overlap with the a-Si:H [24](Fig. 6.16). The replica Asahi texture and the pseudo-random designed patterns both show smooth EQE spectra, indicating that our designed random surfaces are sufficiently random to suppress any particularly strong periodic artifacts. Notably the photocurrent of the $400 \mathrm{~nm}$ pitch cell is also enhanced on the blue side of the spectrum $[21,27,28,140]$.

The same trends with light trapping pattern are visible in all three thicknesses shown in Fig. 8.10, with slight shifting of the peaks for the periodic cells. Figure 8.12 shows the average EQE for the same pattern (400 nm pitch with large particles) on the three different thicknesses. The close overlap on the blue side of the spectrum indicate that the absorption here is independent of thickness (and limited by collection and parasitic ITO absorption). This is consistent with attributing blue side photocurrent enhancements to the top nanostructures. The red side of the spectrum

all show distinct peaks due to the periodicity, with shifts in the wavelength due to the changing thickness of the absorbing layer. Figure 8.11 shows measurements of different cells with the same nanopattern on the $115 \mathrm{~nm}$ i-layer substrate, indicating the reproducibility of the observed features in the EQE spectrum.

The periodic patterns were all made in triplicate, with three different sizes of nanoparticles. Figure 8.13 shows EQE spectra for cells fabricated on patterns with $500 \mathrm{~nm}$ pitch, with small, medium, and large nanoparticles (200, 290, and $310 \mathrm{~nm}$ ). The positions of the peaks on the red side of the band are the same, but the magnitude of the photocurrent varies with size. As predicted by the simulations in Fig. 7.13, the highest photocurrent is measured in the cells with the largest nanoparticles, corresponding to the close-packed position of the ITO scatterers on the top interface. 


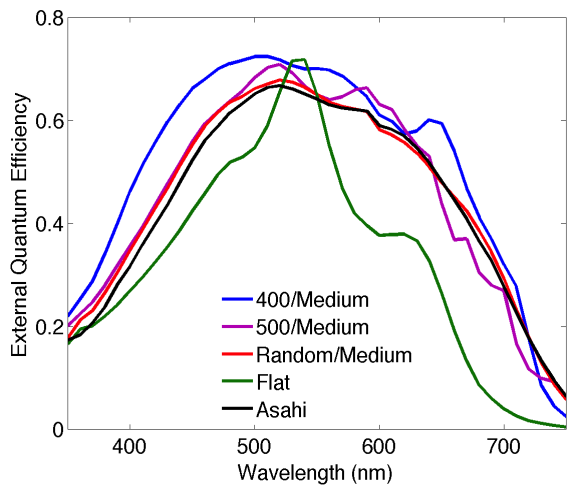

(a) $90 \mathrm{~nm}$ i-layer

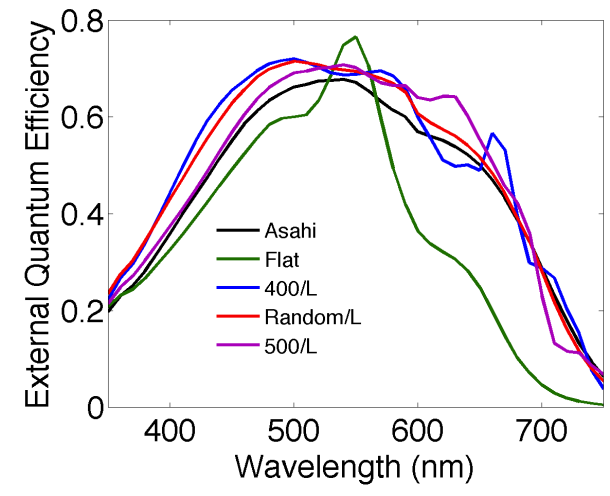

(b) $115 \mathrm{~nm}$ i-layer

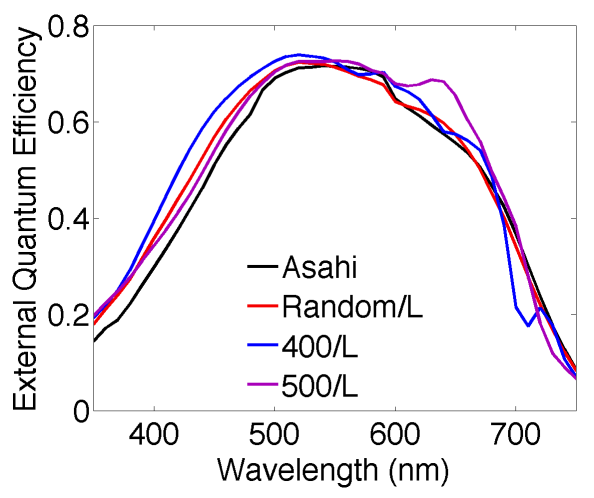

(c) $150 \mathrm{~nm}$ i-layer

Figure 8.10. EQE data on representative cells from other deposition runs. (a) $90 \mathrm{~nm}$ intrinsic regions. (b) $115 \mathrm{~nm}$ intrinsic regions. (c) $150 \mathrm{~nm}$ intrinsic regions. . 


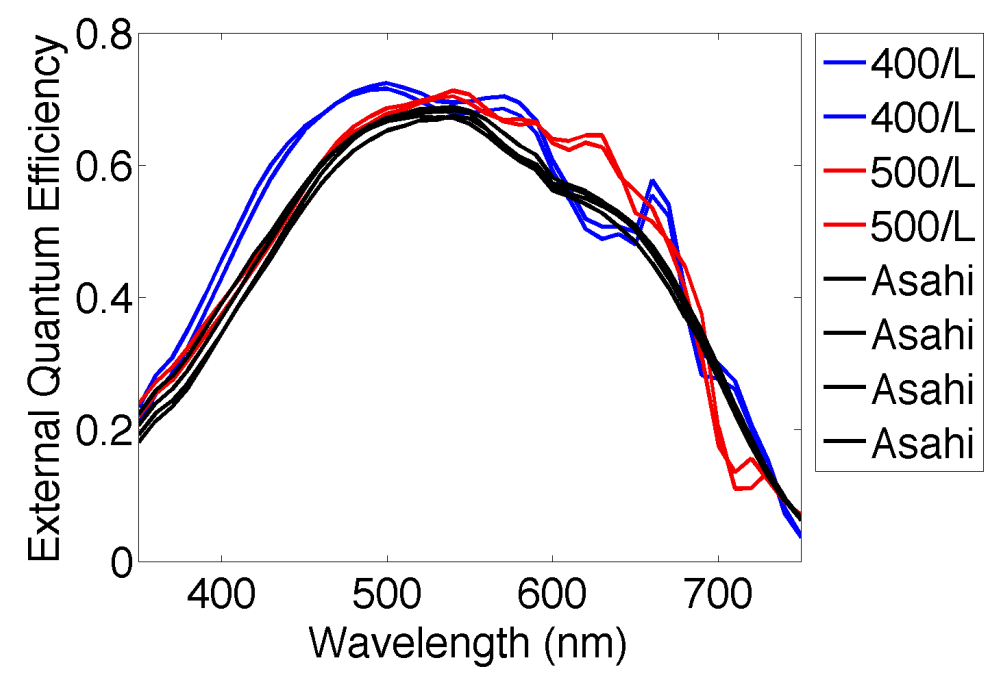

Figure 8.11. Repeatability of EQE data on cells with $115 \mathrm{~nm}$ thick intrinsic regions. Each color trace is a different device on the substrate.

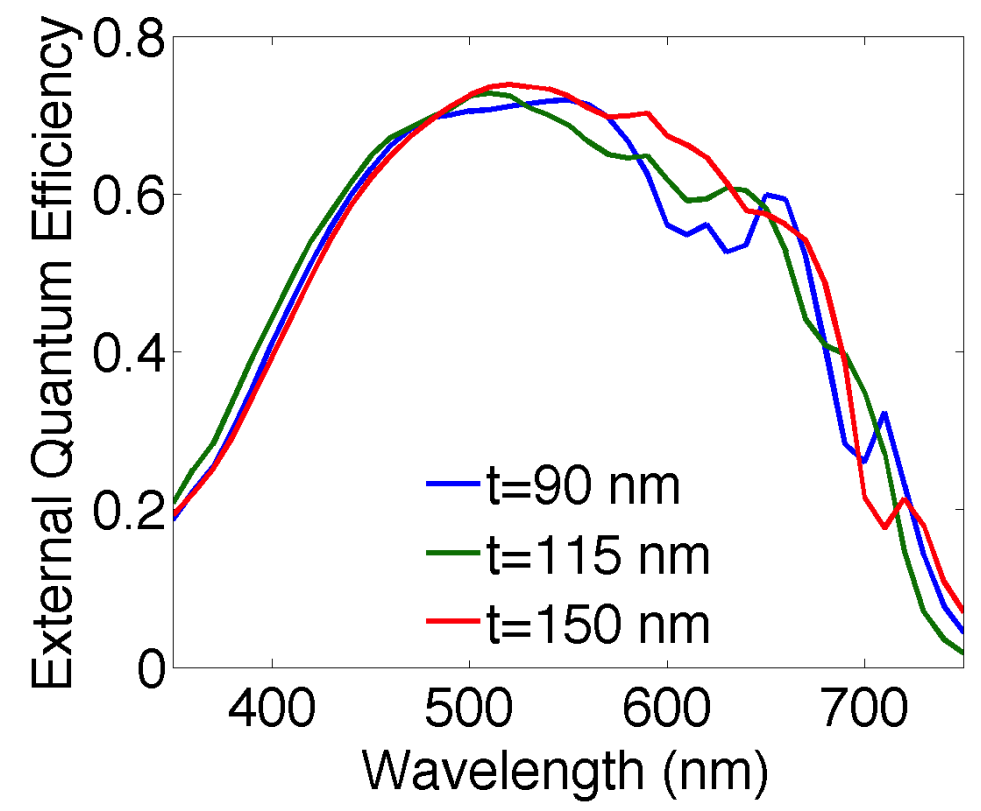

Figure 8.12. Comparison of EQE spectra from cells on $400 \mathrm{~nm}$ pitch nanopatterns with $300 \mathrm{~nm}$ particles from each deposition run. 
163

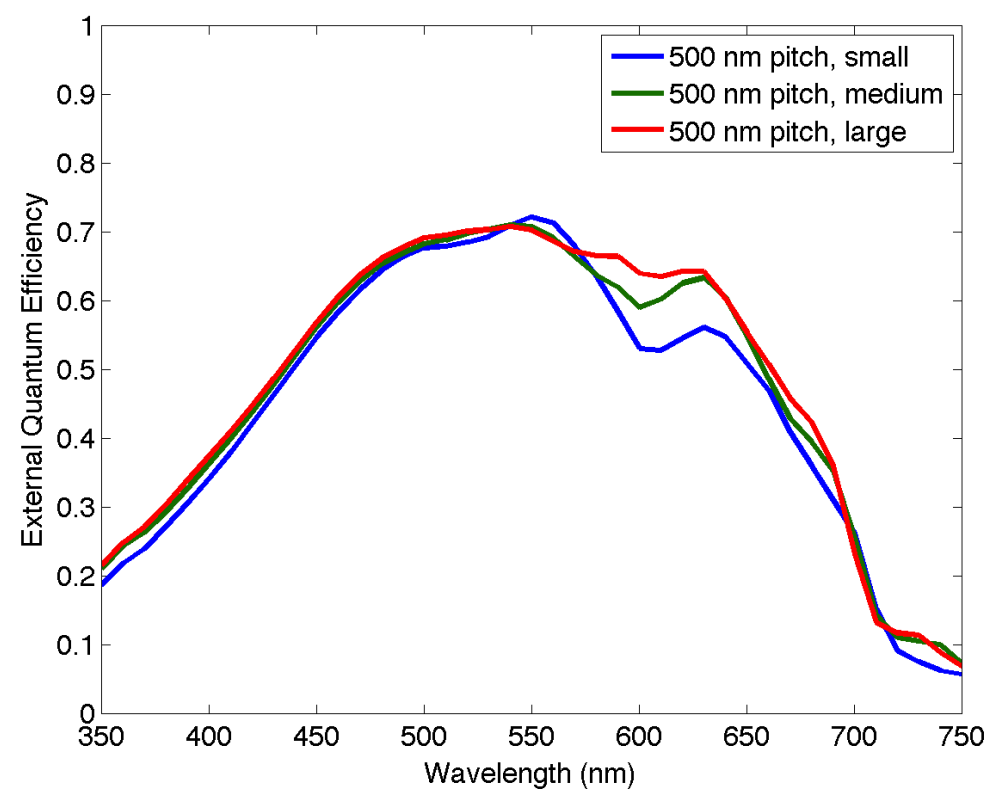

Figure 8.13. EQE spectra for cells with $500 \mathrm{~nm}$ pitch periodic patterns, with varying nanoparticle size, and $115 \mathrm{~nm}$ i-layers.

\subsubsection{Angle-Resolved EQE Measurements}

One of the aims in designing pseudo-random patterns is to achieve an isotropic response. While photocurrent due to waveguide modes is most obvious in mapping out angle-resolved EQE on periodic cells, the sharp angular dependence does decrease the angle-weighted efficiency. In section 6.8 we showed that the Asahi textured cells have an isotropic angle response, but the solar spectrum weighted current is lower than the periodic cells up to $+/-20^{\circ}$. An ideal nanopattern would show high, angleindependent photocurrent (or be mounted to a solar tracking device).

We measured the angle-resolved EQE for the nanopatterned cells at short-circuit conditions $(\mathrm{V}=0)$. Data are shown for $400 \mathrm{~nm}$ pitch and pseudo-random cells with $90 \mathrm{~nm}$ (Figs. 8.14(a) and 8.14(b)) and $150 \mathrm{~nm}$ (Figs. 8.14(c) and 8.14(d)) thickness intrinsic a-Si:H layers. The periodic cells show signatures of waveguide modes in the semiconductor, which shift with changing angle of incidence, as discussed in section 6.8. We observe a higher number of mode branches in Fig. 8.14(c) than in Fig. 8.14(a), due to the higher mode density in the thicker a-Si:H layer. In contrast the engineered random nanopatterns show an isotropic response with angle, indicating 

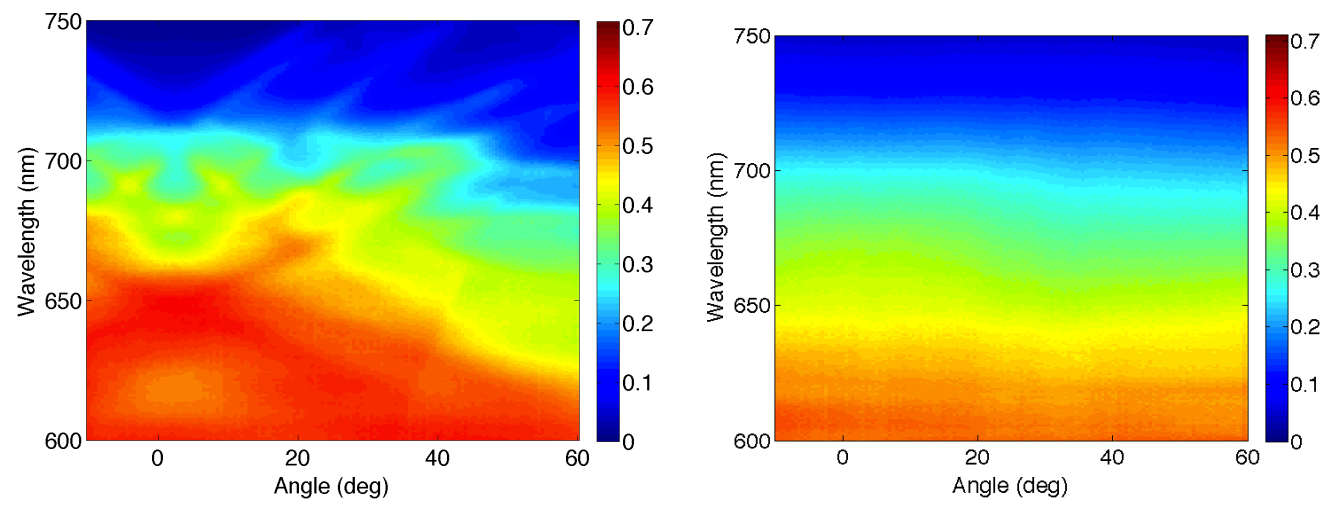

(a) $90 \mathrm{~nm}$ i-layer, $400 \mathrm{~nm}$ pitch periodic pat- (b) $90 \mathrm{~nm}$ i-layer, pseudo-random pattern. tern.
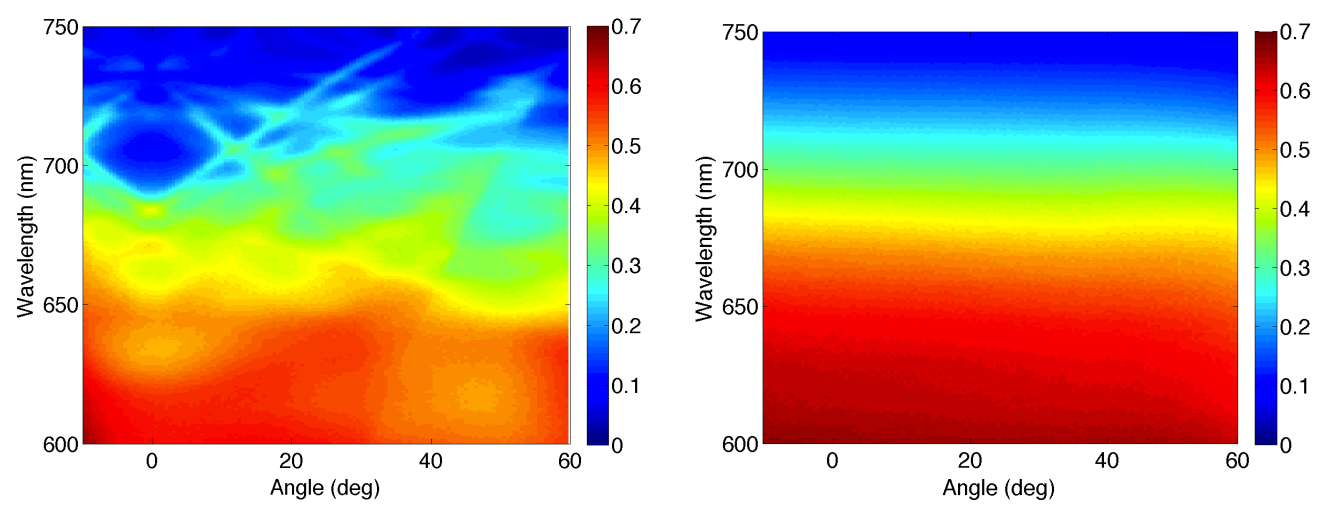

(c) $150 \mathrm{~nm}$ i-layer, $400 \mathrm{~nm}$ pitch periodic (d) $150 \mathrm{~nm}$ i-layer, pseudo-random pattern. pattern.

Figure 8.14. Experimental measurements of angle resolved photocurrent on red side of spectrum. The periodically patterned cells show signatures of coupling to waveguide modes, while the pseudo-random nanopatterns show isotropic angular response. 
that the designed random nanopatterns are sufficiently random to couple to waveguide modes over a broad angle of incidence.

We also took a closer look at the nature of the modes in the periodic cells. In section 6.8 we showed that the mode branches in a cell with a periodic light trapping structure can be correlated with TE and TM modes. To verify this experimentally, we measured the angle-resolved photocurrent for the periodic cells using a polarizer. Figure 8.15 shows maps of the EQE for the same cell shown in Fig. 8.14(a), for the two different polarizations. Different mode branches are clearly visible in the two cases, while Fig. 8.14(a) shows the unpolarized photocurrent.

As one final angle-resolved measurement, we mapped the $\mathrm{V}_{\mathrm{oc}}$ of the cell at $\mathrm{I}=0$. Since $\mathrm{V}_{\text {oc }}$ depends on the current in the cell, this is an alternative way to map out coupling to waveguide modes with good resolution. Figure 8.16 shows good agreement with Fig. 8.14(a), indicating that this does reflect the presence of waveguide modes accurately. While it has also been predicted that the $V_{\text {oc }}$ will increase in a cell that couples strongly to waveguide modes given the decreased entropy of the system, this type of figure does not prove that effect, as $\mathrm{V}_{\text {oc }}$ will also increase with $\mathrm{J}_{\mathrm{sc}}$.

\subsubsection{Simulation of Measured Structures}

As in previous chapters, we simulated the absorption in the a-Si:H with a variety of light trapping nanostructures to correspond to the experimental EQE spectra. The conformal growth of the layers over the Ag nanopatterns is accounted for using the experimental cross sections and AFM data as a guide. For the Asahi texture simulations, the pattern was replicated exactly on each interface rather than accounting for conformal broadening. The pseudo-random patterns were modeled over a $2 \mu \mathrm{m} \times 2$ $\mu \mathrm{m}$ area with periodic boundary conditions. This assumption was verified both via a subsampling routine and with simulation of additional areas of the substrate: the variations are small, indicating that this size simulation does not introduce significant artifacts from the boundary conditions.

The experimental trends correspond well with those predicted from simulation, including the strong enhancement in the blue portion of the spectrum (Fig. 8.17). 
166

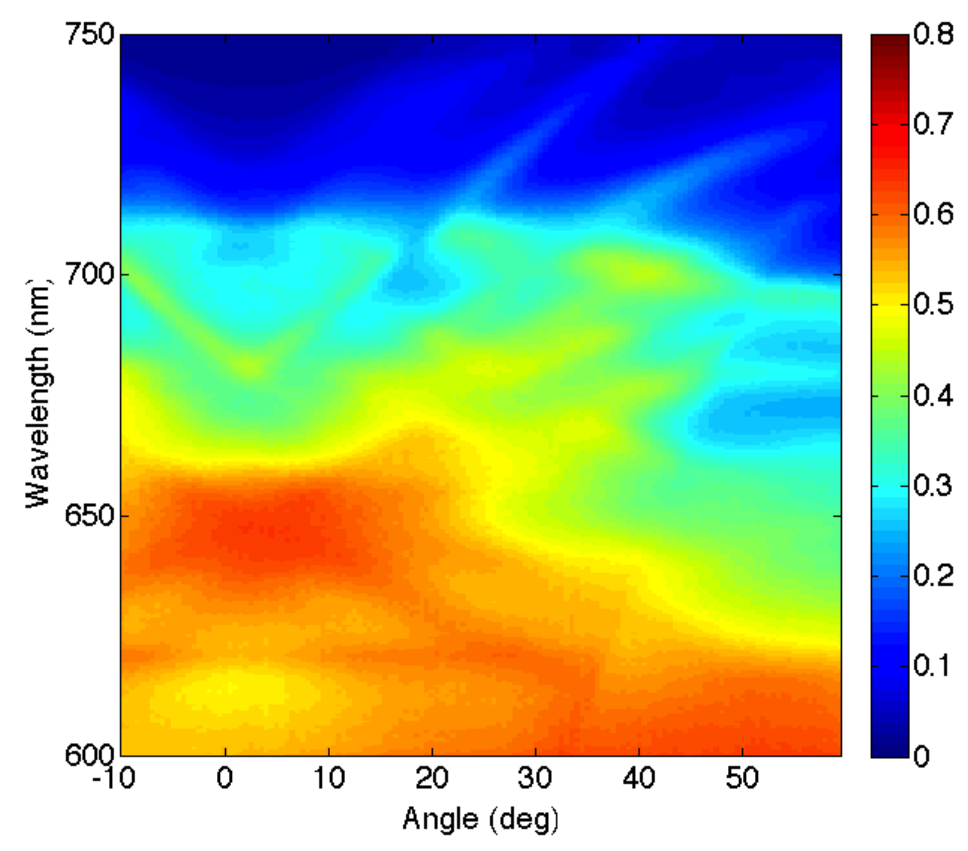

(a)

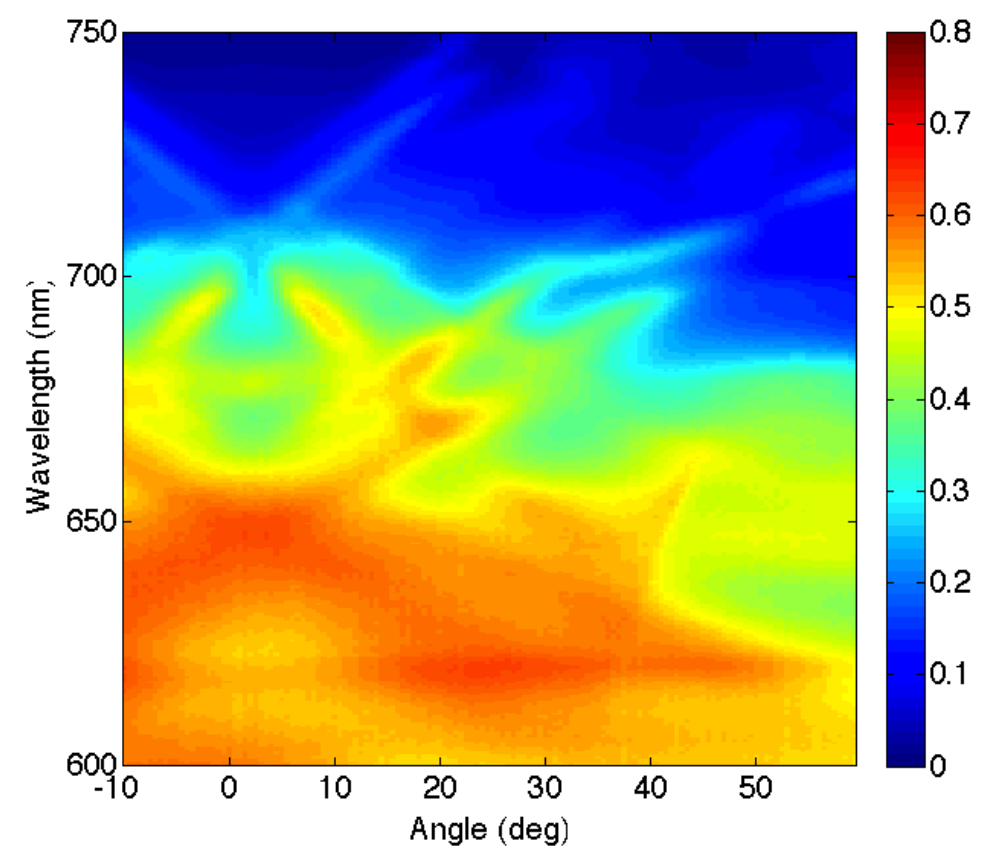

(b)

Figure 8.15. Angle-resolved EQE for cells with $90 \mathrm{~nm}$ thick i-layers on $400 \mathrm{~nm}$ pitch periodic patterns, with polarized incident light. Different mode branches are clearly visible for the different polarizations. 
167

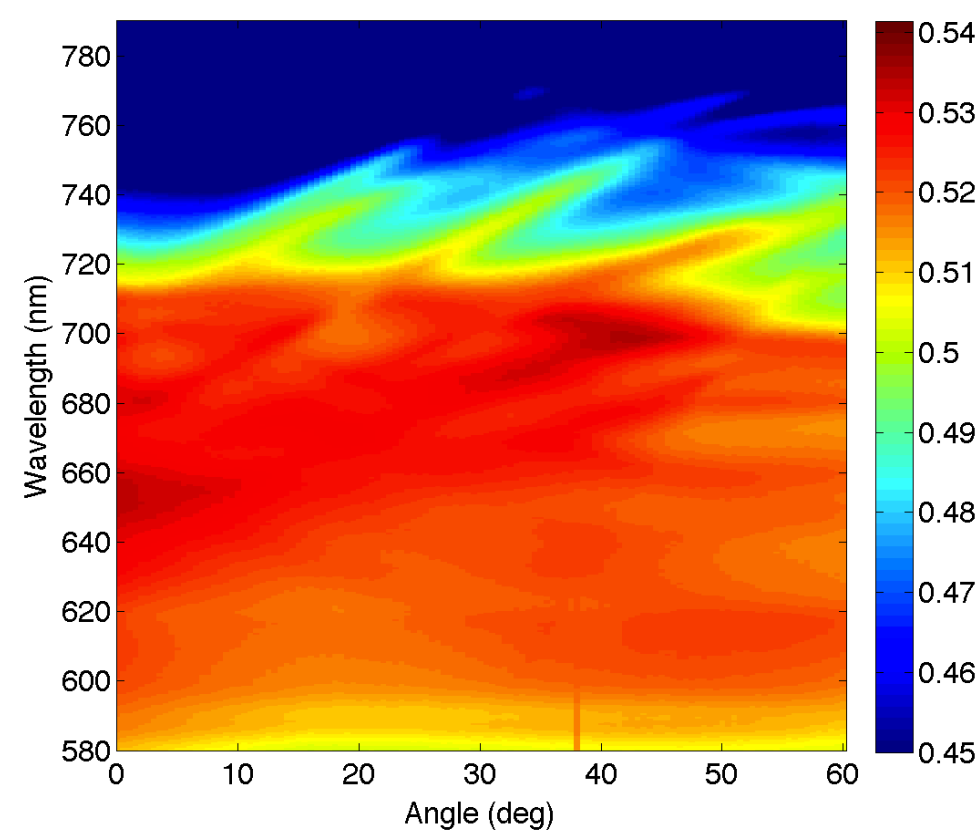

Figure 8.16. Angle-resolved $\mathrm{V}_{\text {oc }}$, measured at $\mathrm{I}=0$, for $400 \mathrm{~nm}$ pitch periodic cell with $90 \mathrm{~nm}$ thick intrinsic region.

Other authors have observed enhancement on the blue side of the spectrum in experimental cells, but it has sometimes been attributed to differences in the p-layer thickness or deposition, which will be dominant on the blue side of the spectrum due to complete light absorption within the top portion of the solar cell. Given that the electromagnetic simulation technique accounts only for optical absorption and not carrier collection, our observed increase on the blue side is likely due to light trapping and not differences in the p-layer thickness or deposition. We attribute the enhanced photocurrent on the blue side of the spectrum to the nanostructured top a-Si:H/ITO surface, which can act as weakly coupled Mie resonators, and will be discussed more in section 8.5. As supporting evidence for this shape effect, we note that the simulated "Random, large" nanopattern also shows enhanced absorption on the blue side of the spectrum, albeit weaker than the $400 \mathrm{~nm}$ pitch periodic pattern. The additional increase in the absorption around $\lambda=400 \mathrm{~nm}$ corresponds with the predicted Bloch mode seen in Fig. 7.13.

At wavelengths where the absorption length is shorter than the scatterer separation, the absorption is mostly due to localized modes, and at wavelengths where 


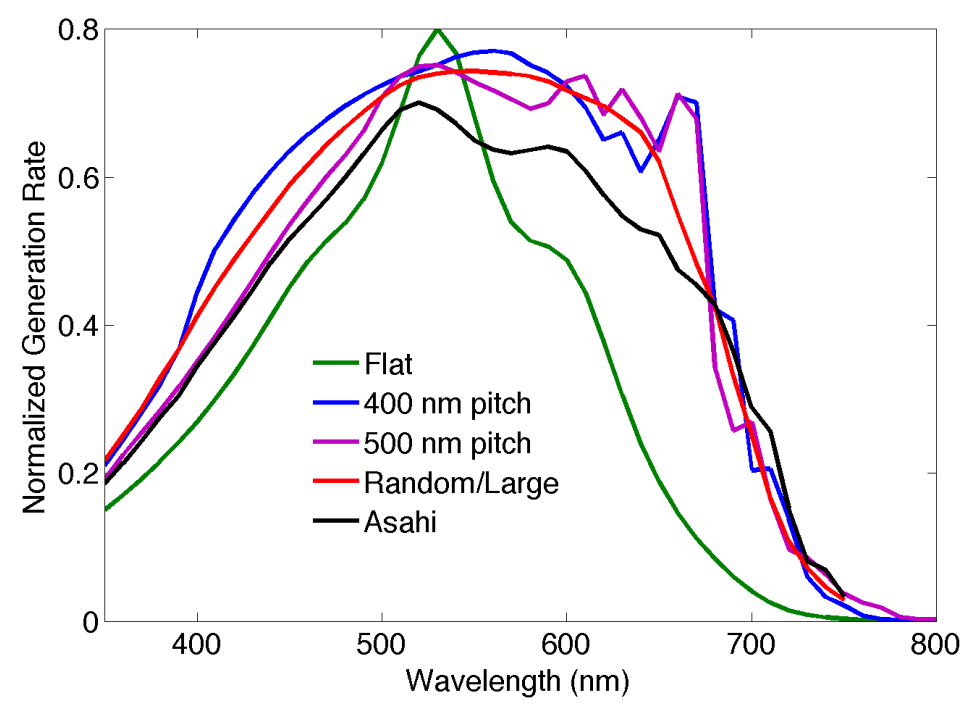

Figure 8.17. Simulated absorption in cells with $90 \mathrm{~nm}$ thick i-layers and various light trapping nanopatterns. The simulations agree well with Fig. 8.10(a).

the absorption length is longer than the scatter separation there will be more of an effect from waveguide modes. Figure 8.18 shows the absorption length in a-Si:H for the optical constants used in our simulations: the absorption length increases dramatically closer to the band edge. Figure 8.19 show photon flux cross sections from simulation for the periodic, Asahi, and pseudo-random patterns at $\lambda=500 \mathrm{~nm}$ and $\lambda=660 \mathrm{~nm}$. At $\lambda=500 \mathrm{~nm}$, the absorption is largely confined over each scatterer, as can be seen by the similarities in the absorption over the a-Si:H. Of course only the absorption in the a-Si:H contributes to photocurrent, but the images in Fig. 8.19 also show the losses in the other layers. These images illustrate one of the key design features of our nanopatterns: the role of curvature within each local scatterer. Plasmonic nanostructures with high frequency shapes (i.e., sharp points) are highly absorbing, leading to parasitic absorption in the metal layer. In contrast, both the periodic and pseudo-random designed nanoarrays control the nanoparticle shape to include only rounded, curved scatterers. At $\lambda=660 \mathrm{~nm}$, more evidence of coupling to waveguide modes is evident. Of course the waveguide modes are easiest to identify in the periodic nanopattern. Variations in the absorption location within the layer are also visible in the pseudo-random and Asahi patterns. 


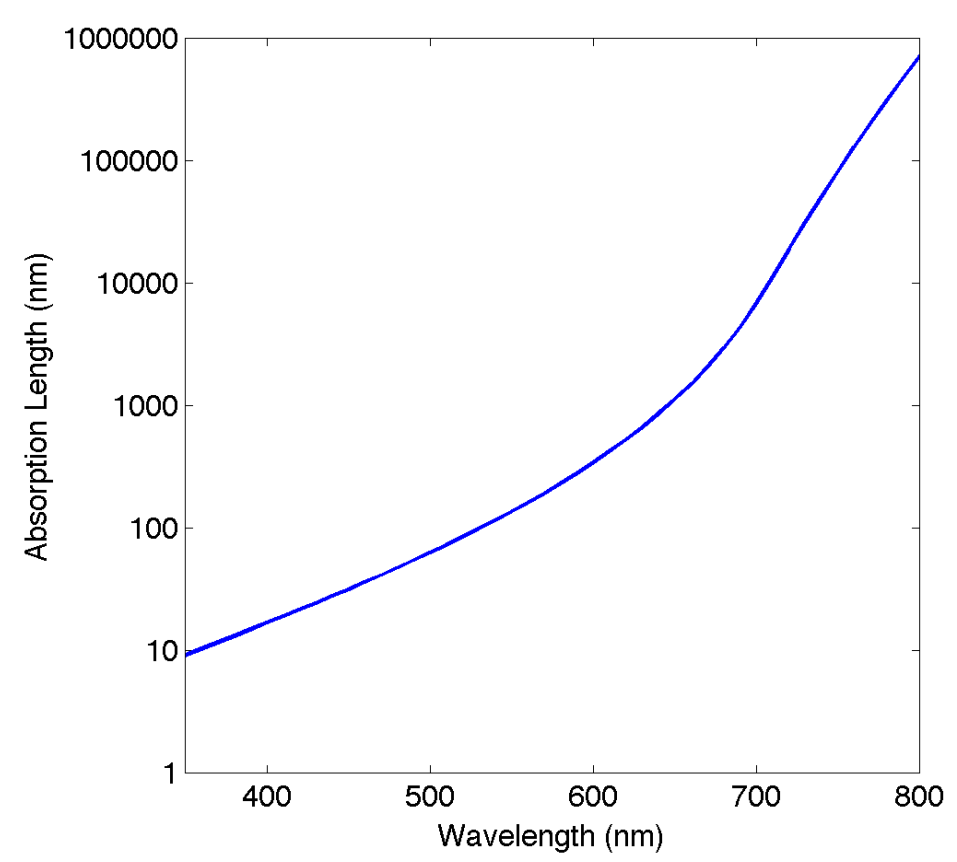

Figure 8.18. Absorption length of a-Si:H vs. wavelength.

\subsection{Connection to PSD Calculations}

As mentioned earlier, to realize coupling to waveguide modes over the broadest possible solar spectral range requires a pattern with flat PSD through the range of spatial frequencies required to couple to the waveguide modes of the a-Si:H and at the same time minimal scattering power in the non-desirable spectral ranges. Within this framework, we can now explain the observed trends in photocurrent and deduce essential design rules. First, we observe that the pseudo-random patterns have higher scattering power than the Asahi texture through the range of optical frequencies required. Indeed, the cells with engineered random patterns show much better cell performance than the Asahi cells (Figs. 8.9 and 8.10). Second, the large nanoparticles have higher scattering power than the smaller nanoparticles, consistent with the better light trapping observed for larger particles in both the periodic and pseudorandom arrays. Third, the periodic array is too narrowband to act as an isotropic light trapping surface. These observations from the PSD are all consistent with the observations from our experimentally measured cells. In summary, the goal of a designed surface is to have a high PSD across the range of spatial frequencies required 
(a)

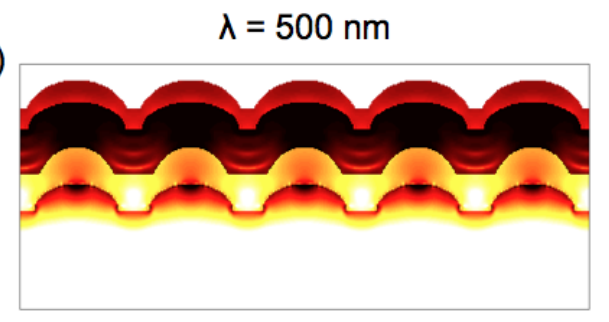

(c)

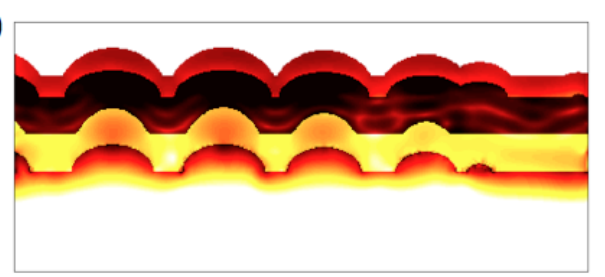

(e)
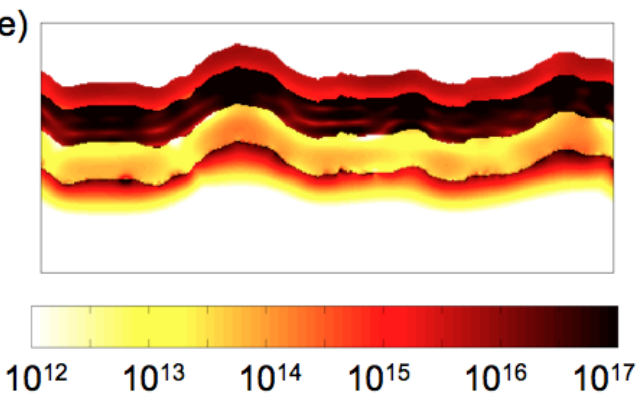

Photon Flux $\left(\mathrm{cm}^{-3} \mathrm{sec}^{-1}\right)$

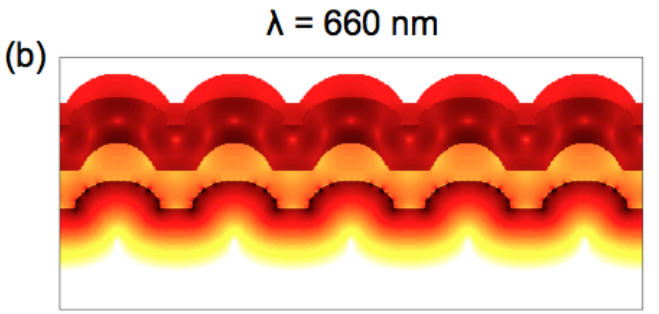

(d)

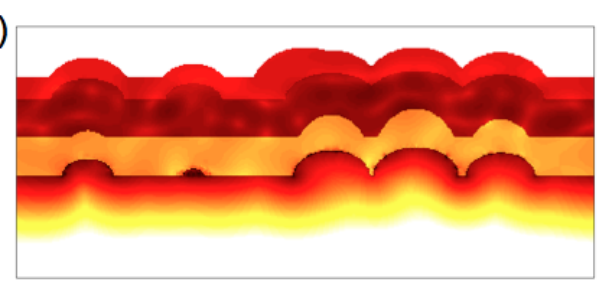

(f)
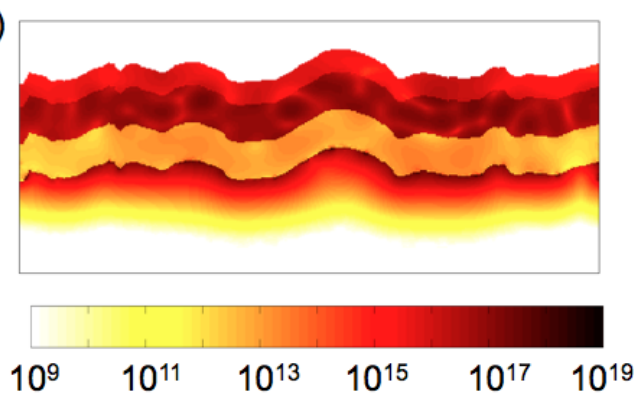

Photon Flux $\left(\mathrm{cm}^{-3} \mathrm{sec}^{-1}\right) \quad 500 \mathrm{~nm}$

Figure 8.19. Photon flux profiles, calculated from simulation, at different wavelengths, for $400 \mathrm{~nm}$ pitch, replica Asahi, and pseudorandom light trapping patterns. 
to couple to the waveguide modes of the a-Si:H. Even though our designed pseudorandom nanopatterns have uniform particle size, unlike those deposited by CVD or etch processes, the result is more effective broadband mode coupling.

\subsection{Mie Scattering from Top Interface}

While the PSD argument explains many of the observations of light trapping on the red side of the spectrum, it does not explain the strong photocurrent enhancement on the blue side of the spectrum. Since the absorption length of a-Si:H is under $100 \mathrm{~nm}$ in the $\lambda=400 \mathrm{~nm}$ to $500 \mathrm{~nm}$ range, the enhanced photocurrent on the blue side must be due to the nanostructuring on the top interface. As further demonstrated below, this is due to the ITO/a-Si:H hemispherical nanopattern acting as weakly coupled Mie resonators, such that incident sunlight is resonantly absorbed in the hemisphere and forward scattered into the a-Si:H region. This enhancement in the UV/blue is consistent with the simulation results shown in Fig. 7.13, and the $400 \mathrm{~nm}$ pitch was chosen a priori from these simulations at it showed the highest solar spectrum integrated absorption .

Spherical (or hemispherical) scatterers can have scattering and absorption cross sections several times their geometrical cross section. While these scattering and absorption cross sections are particularly high for metals (due to the negative coefficient of the permittivity), the cross sections for a semiconductor with high refractive index can also be quite strong. Since the cell layers deposit conformally over the patterned back contact, the features on the top are also quite highly structured, and can be described as a-Si:H hemi-ellipsoids with an ITO coating, as shown in Figs. 8.7 and 8.8.

Figure 8.20 shows the normalized absorption cross section, defined as the ratio between absorption and geometrical cross section $\left(Q_{a b s}=\frac{\sigma_{a b s}}{\sigma_{\text {geom }}}\right.$ calculated from simulations) for the blue side of the spectrum. The calculations were done using FDTD simulation for a $350 \mathrm{~nm}$ diameter with an $80 \mathrm{~nm}$ ITO coating. A semi-infinite a-Si:H substrate is considered in the calculation with absorption set to zero in the substrate 


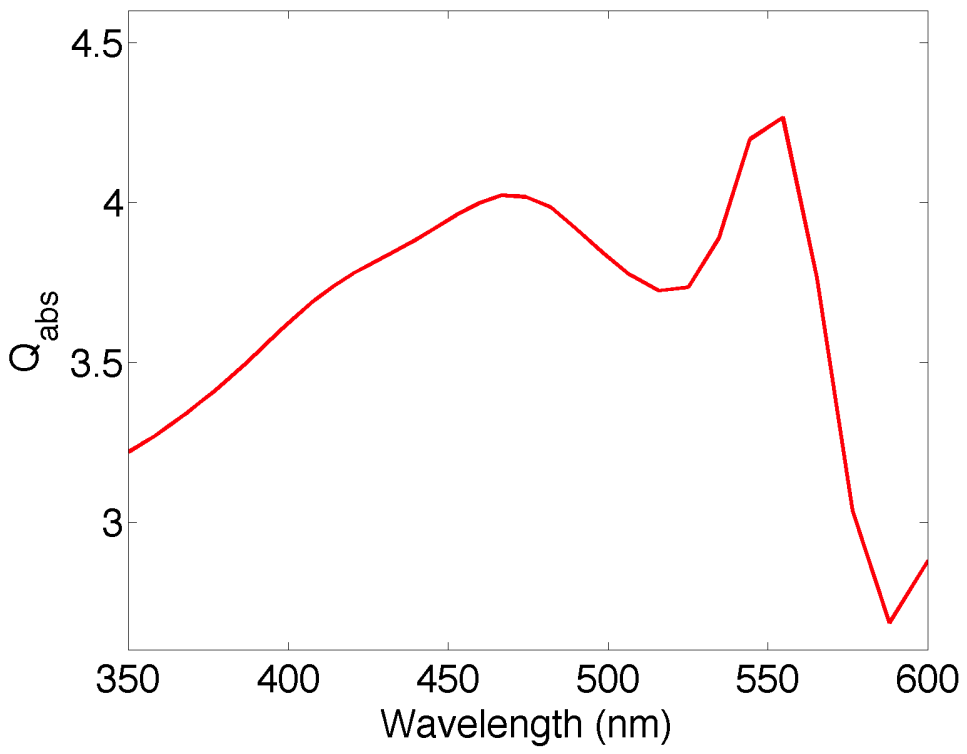

Figure 8.20. Calculated normalized absorption cross section $\left(Q_{a b s}\right)$ for a hemi-ellipsoidal a-Si:H/ITO scatterer on a semi-infinite nonabsorbing a-Si:H substrate.

to separate the effect of absorption in the particle from transmission. The figure shows two clear absorption resonances with a peak $Q_{a b s}$ near 4 . The effective interaction cross section of these scatterers is $3-4$ times their geometrical area over a broad UV/blue spectral range and thus leads to enhanced incoupling of light at these wavelengths, for which light would otherwise be strongly reflected. The resonance linewidth is larger for the lowest wavelength resonance, which is due to the stronger absorption in the a-Si:H at shorter wavelengths.

Figure 8.21 shows the calculated photon flux from the simulations at various wavelengths in the blue region of the spectrum. The top row shows data for a simplified geometry similar to that in Fig. 8.20, except with an absorbing, semi-infinite a-Si:H substrate. Data for the actual solar cell geometry are shown in the second row. For $\lambda=350-450 \mathrm{~nm}$ the absorption profiles are clearly similar between the simplified structure and the actual layer structure, indicating that the absorption occurs in the top surface structure. Figures 8.21(d, e) shows how for longer wavelengths, $\lambda=500$ $550 \mathrm{~nm}$, light is concentrated inside the hemisphere and coupled downward into the substrate. In the corresponding structure in (i) similar features are seen near the top 


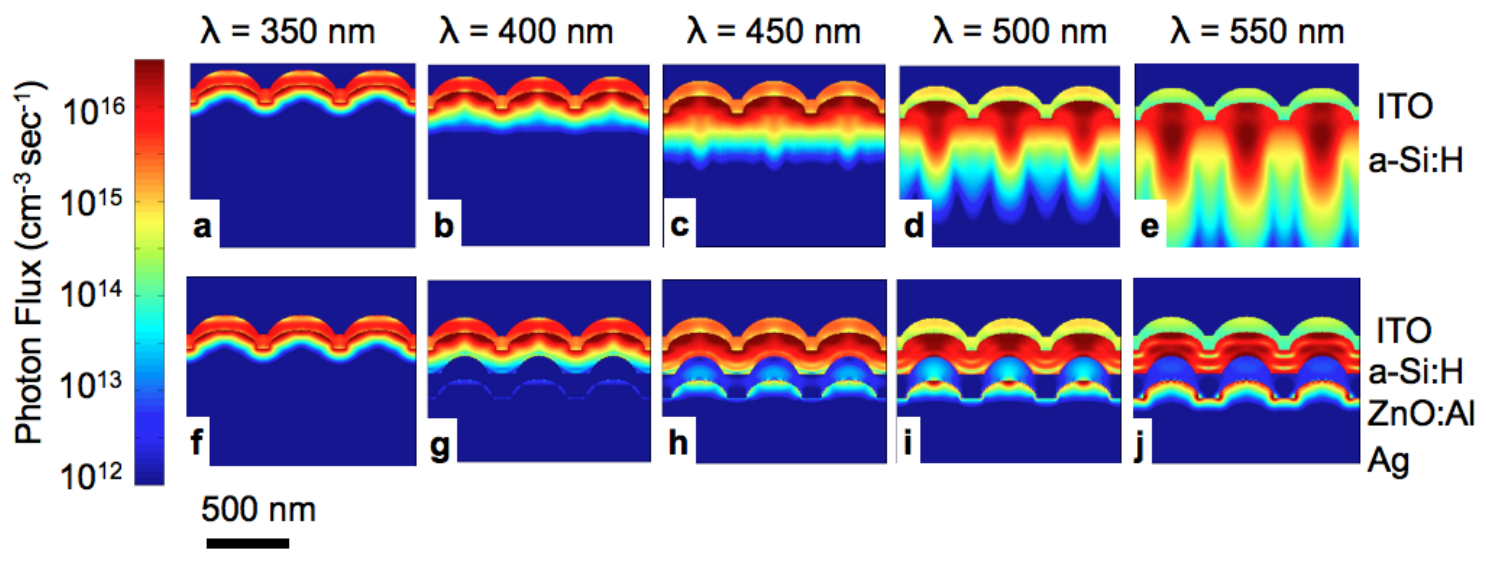

Figure 8.21. Photon flux profiles, calculated from simulation, at different wavelengths. (a) - (e) are for a conceptual device with a$\mathrm{Si}: \mathrm{H} /$ ITO nanostructuring on a semi-infinite a-Si:H substrate. (f)-(j) are for the actual device structure. The intrinsic a-Si:H layers are $90 \mathrm{~nm}$ thick.

of the a-Si:H scatterer. At the Mie resonance at $\lambda=550 \mathrm{~nm}$ the light is strongly coupled from the scatterer into the substrate

\subsection{Conclusions}

In summary, systematic study and design of pseudo-random arrays of resonantly scattering nanoparticles allow for both an understanding of the ideal random patterns for broadband, isotropic angular response and the realization of high efficiency devices in extraordinarily thin semiconductor regions. Key to the design is an integrated understanding of spatial frequencies and curvature of nanoscale scatterers that form plasmonic backreflectors coupling to waveguide modes and weakly coupled surface Mie scatterers coupling to localized surface modes. While the diameters and pitch of the front and back surface patterns in this study were designed with a one-step, conformal deposition process in mind, it is possible that decoupling the pattern formation at the two interfaces will lead to higher overall efficiencies, extending the applicability to nonconformal semiconductor depositions. While focused here on a-Si:H, the principles presented here are potentially applicable to other solar cell materials systems. 


\section{Chapter 9}

\section{Summary and Outlook}

The aim of this thesis is to explore the use of plasmonics in photovoltaics, and particularly to understand how the control of light on the nanoscale can be used to enhance performance in ultrathin-film solar cells. We developed and used novel computational tools such as the FMSA method and the direct calculation of absorption to study coupling to waveguide modes and understand the effects of nanophotonic design on absorption. We also used a large-scale nanopatterning method, SCIL, to pattern large-area substrates with carefully designed nanostructures. This technique enabled comparisons between designed nanostructures and randomly textured surfaces, showing first that periodic nanostructures can have enhanced photocurrent beyond randomly roughened surfaces, and to develop an understanding of the roles of spatial frequency and spatial curvature in light trapping structures. This thesis shows that light management is a rich area for study in photovoltaics, and that photovoltaics is a promising arena for plasmonics.

\section{$9.1 \quad$ Summary}

We began in Chapter 4 with an analysis of mode coupling from individual nanostructures across a range of wavelengths and incident angles. Using a simplified solar structure of a thin semiconductor on an Ag film containing a single groove or ridge to overcome the momentum mismatch between incident light and waveguide modes, we developed a simulation toolbox based on Fourier decomposition to study the coupling from different shapes to particular waveguide modes. We found that the coupling across the solar spectrum could be described by the convolution of different types of 
resonances, including the coherent Fabry-Perot resonances of the film determined by its thickness, the individual nanostructure's resonance determined by its size, shape, and dielectric environment, and the SPP resonance of the semiconductor-metal combination. We also showed that individual nanostructures scatter outward like dipoles, and thus are also isotropic with respect to angle of incidence. This chapter showed that isolated nanostructures can lead to surprisingly high absorption enhancements across a broad range of wavelengths and angles of incidence due to coupling to waveguide modes.

We next studied the properties of the individual modes, in Chapter 5. This chapter showed that SPP modes in c-Si can be quite lossy, but that much higher absorption enhancements can be achieved by coupling to photonic modes. In the diffusion-model described here, coupling to modes is determined by the effective height of a point dipole within the semiconductor, coupling most strongly to modes with significant amounts of power at the same vertical height. Combined with understanding from Chapter 4 about the role of nanostructure shape in selective mode coupling, this showed that ridge nanostructures are the most promising structure.

Chapter 6 showed that SCIL is a versatile and effective method for patterning surfaces for photovoltaic applications. We first used a patterned hole-array electrode to show that a patterned back electrode strongly enhances photocurrent over a flat reference cell. We then designed a master substrate with several different arrays of nanopillars, allowing for one deposition run to study many different nanopatterns. We showed that periodic nanostructures can lead to enhanced performance beyond that measured in randomly textured reference cells. We also verified through angle-resolved photocurrent measurements that the enhanced photocurrent is due to coupling to waveguide modes in the semiconductor. This demonstrated that careful nanostructure design could be used in ultrathin-films to surpass the light trapping achievable with randomly deposited textures.

In chapter 7 , we showed that numerical calculation of the absorption in a nanostructured solar cell compares well to the measured external quantum efficiency, verifying that this is a powerful tool for optimization and understanding of absorption 
processes in nanophotonic photovoltaics. We studied the effect of pitch, and showed that the absorption enhancement is strongly dependent on packing fraction, and predicted a significant broadband enhancement by moving to the closest packed position of a square array. From comparisons of the generation rate maps of randomly textured and periodic cells, we also identified the importance of spatial curvature of each nanostructure: sharp metallic features lead to high losses, while rounded nanostructures scatter more light into the photocurrent-generating semiconductor.

Finally, we combined these ideas to study the role of spatial frequency in nanopatterns, and achieve broadband and isotropic response. First, by coating the patterns conformally, we take advantage of the localized resonances of the a-Si:H/ITO top-side structures to enhance absorption on the blue side of the spectrum, while the red side is enhanced by coupling to waveguide modes. We then proposed a general framework for evaluating random textures, based on the power spectral density of the surface, where we seek to maximize the PSD through the range of spatial frequencies that couple to the modes of a-Si:H. Applying this design principle to pseudo-random pattern arrays leads to isotropic angular response, as the patterns are sufficiently random to be invariant with respect to angle of incidence. The combination of optimized spatial curvature, pattern arrangement, and conformal overcoating produced high efficiency devices. Moreover this experiment showed a new way to design random surfaces, without restriction to native texturing or etch processes, but entirely by design.

\subsection{Outlook}

\subsubsection{Fabrication of p-i-n Cells}

Most commercial a-Si:H solar cells are made in the superstrate configuration rather than the substrate configuration, meaning that the p-layer is deposited first on a TCO surface (as opposed to the n-layer first on a $\mathrm{ZnO}: \mathrm{Al} / \mathrm{Ag}$ surface). It is likely straightforward to reconfigure our fabrication process to work with these p-i-n devices. As shown in Fig. 9.1, the SCIL process could be used to pattern silica sol-gel on glass, 


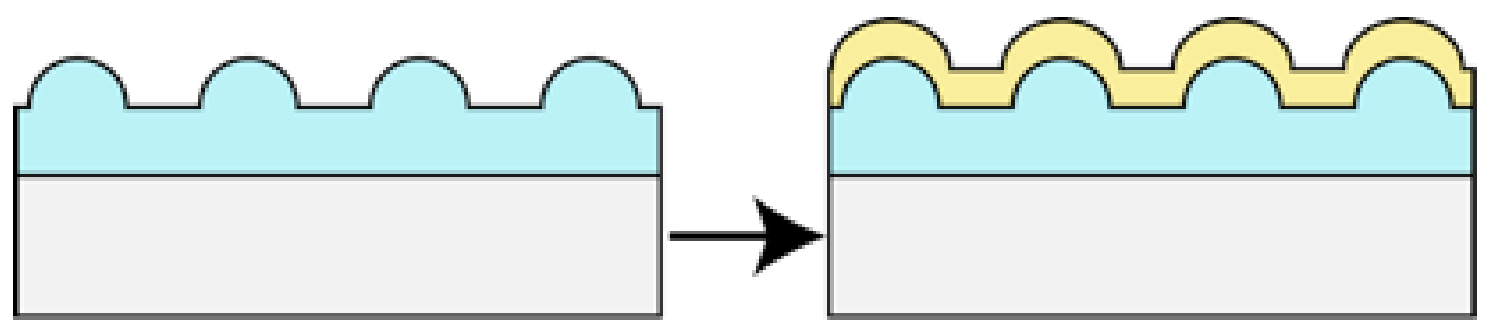

Figure 9.1. Fabrication of superstrates for p-i-n devices. Silica solgel on glass is imprinted, then overcoated with a TCO to form a superstrate.

which could then be coated in a TCO such as ITO. Cells could then be grown over the TCO, with the pattern transferring through to the back metallic contact. This arrangement does require transparency of the silica sol-gel across the whole spectral range of interest. In addition to nanophotonic design, this could also be used with the random structure replication discussed in Chapter 8: essentially any material with an interesting native texture could be used as a master for stamp fabrication, transferred into silica sol-gel, and then overcoated with a more desirable TCO.

\subsubsection{Application to Multijunction Cells}

While single junction a-Si:H solar cells are a useful platform for understanding light trapping, and the work in this thesis showed significantly higher efficiencies while accessing more stable configurations, commercial thin-film Si solar cells most likely need efficiencies in the $10 \%-13 \%$ range to be competitive with CIGS and CdTe. Multijunction cells based on different compositions of a-Si:H and $\mu c-\mathrm{Si}$ are capable of reaching these efficiencies, and thus may be more commercially promising. Light trapping in $\mu \mathrm{c}-\mathrm{Si}$ is particularly important: $\mu \mathrm{c}-\mathrm{Si}$ has optical properties more similar to c-Si, and is a much weaker absorber than a-Si:H. The typical rough surfaces used in other thin film $\mathrm{Si}$ cells are also difficult to use with $\mu \mathrm{c}-\mathrm{Si}$, as the columnar grain growth leads to cracking and void formation over certain rough features.

The computational techniques described in this thesis could be used in conjunction with information about the growth of $\mu \mathrm{c}-\mathrm{Si}$ to design a light trapping surface with lower overall surface roughness and preferable nanostructure shape, but preserve a 

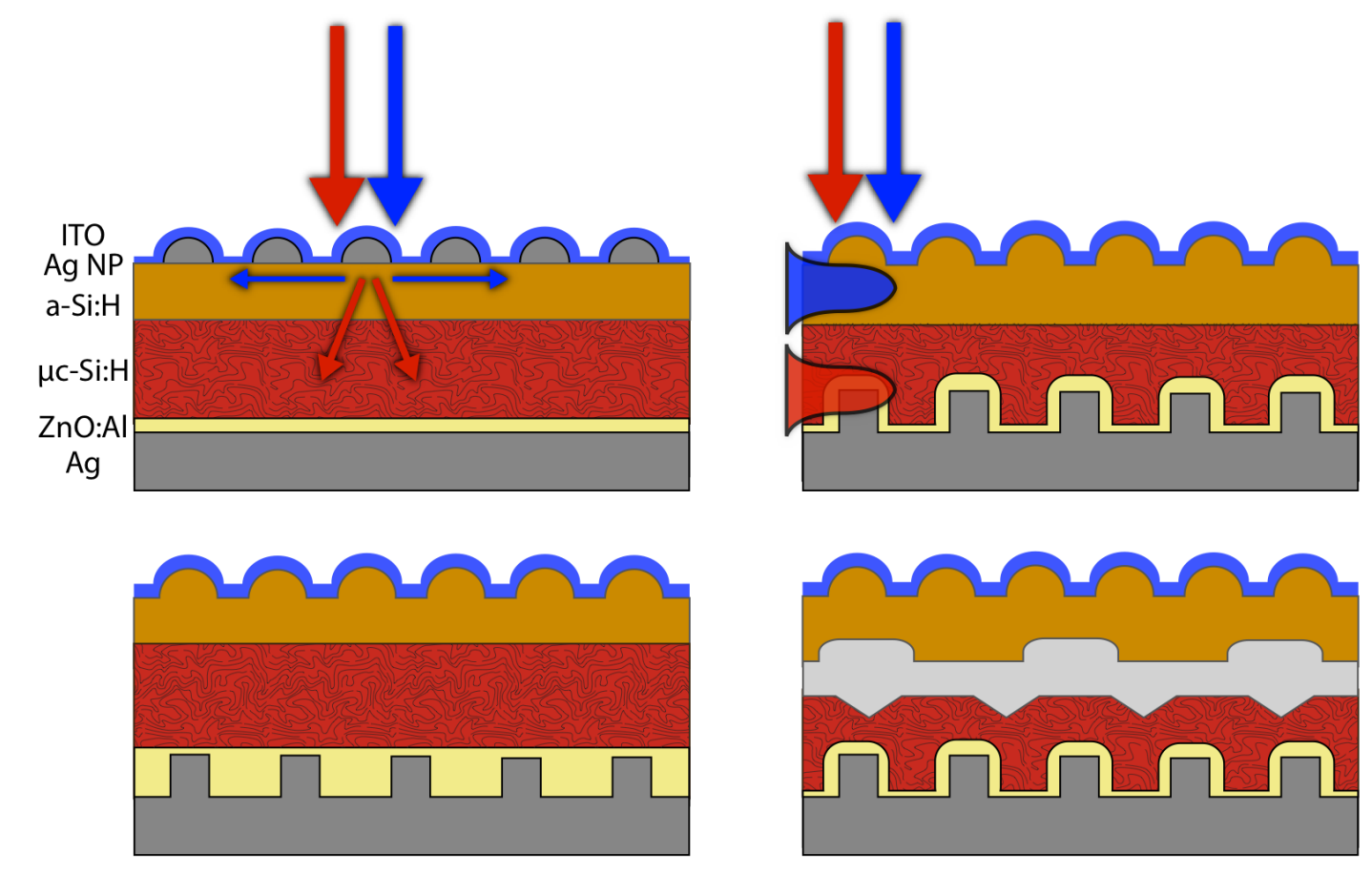

Figure 9.2. Schematic designs of nanostructured tandem thin-film Si solar cells. 
high degree of light trapping. Furthermore, in the designs discussed in chapter 8 , while a broadband photocurrent enhancement is achieved by integrating the top and back structures in a one-step conformal coating process, it is possible that higher levels of enhancement could be achieved by decoupling the two interfaces, with the back metal patterns designed for red-side response and the top semiconductor patterns designed for blue-side response. The nanopatterns then serve as spectrum splitters, affecting light of specific colors more strongly.

Several schematic designs for nanostructured tandem Si cells are shown in Fig. 9.2 building on these ideas. In the first, metal nanostructures on the top surface of a multijunction cell are used to scatter light of different colors with different angular distributions, directing blue light to the higher bandgap a-Si:H cell and red light to the lower bandgap $\mu \mathrm{c}-\mathrm{Si}$ cell. The second design is similar to the a-Si:H cells discussed in Chapter 8, except that the top and back patterns are independent from each other. The higher index a-Si:H cell can use semiconductor nanostructuring to enhance absorption on the blue side of the spectrum, while the back metal patterns can enhance red absorption in the $\mu \mathrm{c}-\mathrm{Si}$ cell. The third design is similar, but shows one potential way for dealing with the issues of $\mu \mathrm{c}-\mathrm{Si}$ growth: the $\mathrm{ZnO}: \mathrm{Al} / \mu \mathrm{c}-\mathrm{Si}$ could be made planar, such that the metal surface underneath provides light trapping but the growth surface is flat. The fourth design shows a more extreme case of light manipulation, adding an intermediate reflector between the a-Si:H and $\mu c-\mathrm{Si}$ cells. Asymmetric intermediate reflectors (consisting of materials such as $\mathrm{ZnO}: \mathrm{Al}$ ) have recently been discussed as a method for improving light absorption in multijunction Si cells, although to date only random textures have been studied. This intermediate reflector could potentially be designed as another spectrum splitting interface, with one side providing some red-side enhancement for the a-Si:H cell and the other blue or green-side enhancement for the $\mu \mathrm{c}-\mathrm{Si}$ cell.

\subsubsection{Application to other Materials Systems}

Most of the absorption enhancement from waveguide modes in the experimental devices discussed were due to photonic waveguide modes rather than SPP modes. Chap- 


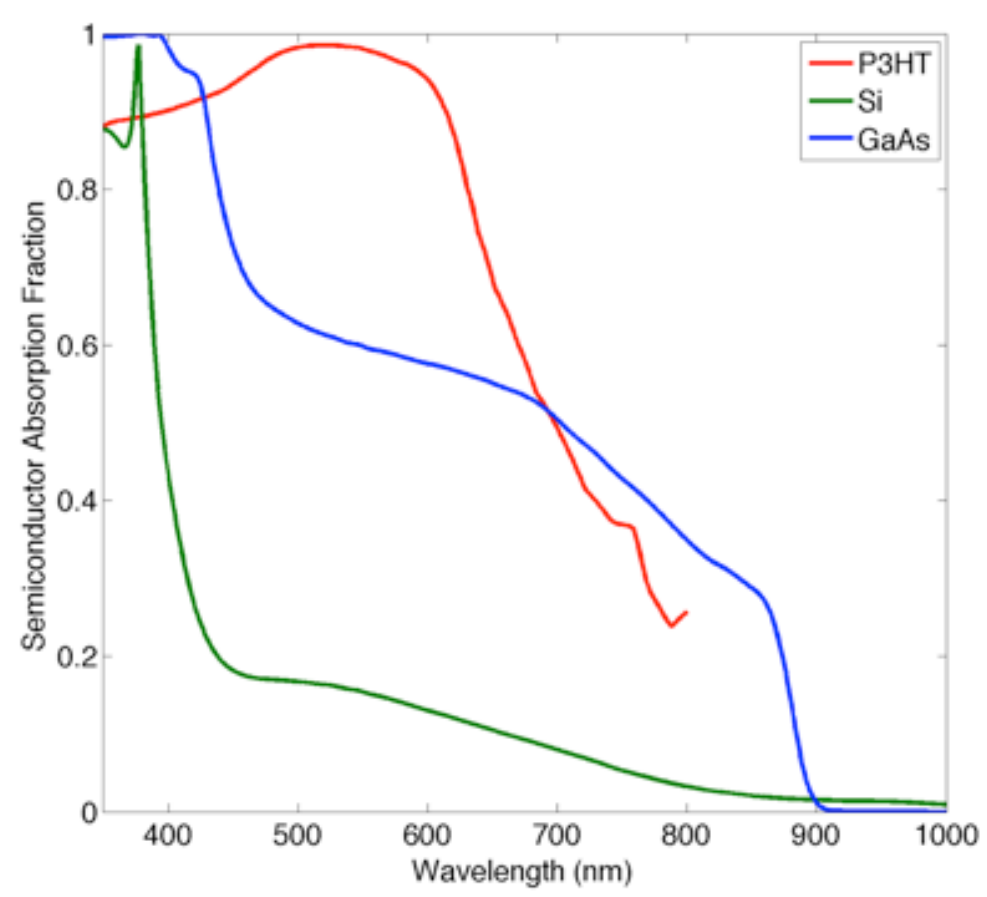

Figure 9.3. Fraction of power in an SPP mode absorbed in semiconductor. The metal is Al.

ter 7 showed that one of the roles of the $\mathrm{ZnO}$ :Al layer is to diminish coupling to the SPP mode, and the calculations in Chapters 4 and 5 showed that these modes are quite lossy in c-Si. Nevertheless, the high degree of confinement in SPP modes is attractive for achieving extraordinarily thin solar cells, and particularly for severely collection-limited materials.

The ratio of useful absorption to loss in an SPP mode depends strongly on the materials used. Figure 9.3 shows a simple evaluation of this loss ratio for a few different sets of materials. The calculation assumes a semi-infinite semiconductor on top of semi-infinite $\mathrm{Al}$, and shows only the fraction of power in a propagating SPP mode that is absorbed in the semiconductor (with the remainder absorbed in the metal). For c-Si, a very small fraction of the power is absorbed in the semiconductor: below $20 \%$ from 400 to $1100 \mathrm{~nm}$. The ratio is quite a bit more promising for GaAs and for polythiophene (P3HT), a common polymer solar cell material. P3HT has a significantly lower refractive index, and so the SPP will extend quite far through the material: calculations for finite thickness P3HT would need to verify that similar 


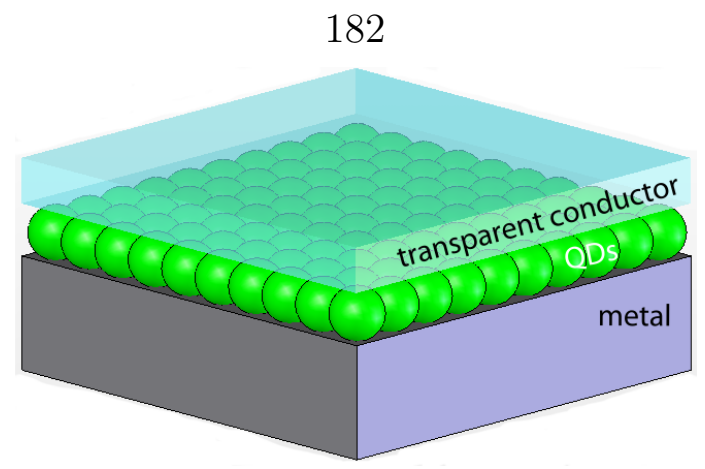

Figure 9.4. Schematic of a quantum dot solar cell.

behavior occurs in thin films. Another alternative shown in Fig. 9.4 is quantum dot (QD) solar cells. Quantum dots are strongly absorbing and their bandgap can be tuned across the solar spectrum by changing the size of the particle, but transport through QD films is difficult. Combining QD solar cells with SPP modes could potentially allow for optically thick absorption in layers as thin as a single monolayer.

\subsubsection{Effect of Nanostructures on Voc and FF}

Most of the work in this thesis correlated the photocurrent to different light trapping structures, as photocurrent is the cell parameter most strongly related to path length. Overall cell efficiency, however, is the product of the $\mathrm{J}_{\mathrm{sc}}, \mathrm{V}_{\mathrm{oc}}$, and $F F$, and there are effects of nanophotonic light trapping on the latter parameters as well. As discussed earlier, $\mathrm{V}_{\mathrm{oc}}$ is related to the ratio of the photocurrent to the dark current, and decreasing the volume of material will decrease the dark current and improve $\mathrm{V}_{\mathrm{oc}}$. The $F F$, however, may decrease under such circumstances due to the increased probability of recombination in concentrated volumes. A detailed study of changes in $\mathrm{V}_{\text {oc }}$ and FF with various nanopatterns may give more insight into the ideal nanopattern for cell efficiency, not just photocurrent. The inhomogeneous absorption profiles mapped in Chapters 7 and 8 also have potential for extension: given the variation in absorption, the device physics of a cell with nanophotonic light trapping may be different from either a flat cell or a randomly textured surface. Indeed, the profile may even be more homogeneous than a randomly textured thin film solar cell. If this process were well-understood, then unusual device architectures not possible with standard 
absorption profiles could be designed.

There is also an opportunity to study material deposition over different types of nanostructures, and particularly how this affects $\mathrm{V}_{\mathrm{oc}}$. Certain types of rough surfaces are known to increase shunting, and to lead to the formation of voids in the material that also degrade $V_{\text {oc }}$. A thorough statistical analysis of shunting rates and $V_{\text {oc }}$ over surfaces with lower overall roughness and controlled nanostructure shape may show that designed surfaces also improve semiconductor quality.

\subsection{Conclusions}

From 2008 to present, there has been growing interest in the use of plasmonics in photovoltaic devices for a wide variety of absorbing materials and geometries. Light management in solar cells was a previously neglected area, with limitations on the types of random structures that could be fabricated, as well as a pervasive conventional wisdom that randomly deposited structures would lead to higher enhancements than designed features. As a result of this work, we now have computational methods that can be used to study and design nanophotonic surfaces for light trapping in solar cells, as well as demonstration of large-area, controlled nanopatterns to experimentally study the role of nanostructure shape and arrangement on solar cell performance. While applied to thin-film Si solar cells, the techniques are general and could be applied to many other types of devices: solar cells of other materials, as well as photodetectors and sensors. Moreover, this work shows the potential for designed nanostructures in controlling the flow of light through photovoltaics, opening the door to future unusual geometries and structures. 


\section{Bibliography}

[1] N. S. Lewis. Powering the planet. MRS Bulletin, 32(10):808-820, 2007. 1

[2] A. Luque and S. Hegedus. Handbook of Photovoltaic Science and Engineering. John Wiley \& Sons, England, 2003. 2

[3] M. A. Green. Third generation photovoltaics: solar cells for 2020 and beyond. Physica E-Low-Dimensional Systems \& Nanostructures, 14(1-2):65-70, 2002. 2

[4] A. Romeo, A. Terheggen, D. Abou-Ras, D. L. Batzner, F. J. Haug, M. Kalin, D. Rudmann, and A. N. Tiwari. Development of thin-film Cu(In,Ga)Se-2 and CdTe solar cells. Progress in Photovoltaics, 12(2-3):93-111, 2004. 2

[5] M. A. Contreras, B. Egaas, K. Ramanathan, J. Hiltner, A. Swartzlander, F. Hasoon, and R. Noufi. Progress toward 20\% efficiency in $\mathrm{Cu}(\mathrm{In}, \mathrm{Ca}) \mathrm{Se}-2$ polycrystalline thin-film solar cells. Progress in Photovoltaics, 7(4):311-316, 1999. 2

[6] J. Schmidtke. Commercial status of thin-film photovoltaic devices and materials. Optics Express, 18(19):A477-A486, 2010. 2, 3, 10

[7] US Geological Survey. Mineral Commodity Summaries. Technical report, Washington DC, 2009. 2, 7

[8] B. A. Andersson. Materials availability for large-scale thin-film photovoltaics. Progress in Photovoltaics, 8(1):61-76, 2000. 2, 7

[9] C. Wadia, A. P. Alivisatos, and D. M. Kammen. Materials Availability Expands the Opportunity for Large-Scale Photovoltaics Deployment. Environmental Science $\&$ Technology, 43(6):2072-2077, 2009. 2, 7 
186

[10] A. L. Fahrenbruch and R. H. Bube. Fundamentals of Solar Cells: Photovoltaic Solar Energy Conversion. Academic Press, London, 1983. 4

[11] R. E. I. Schropp and M. Zeman. Amorphous and Microcrystalline Silicon Solar Cells: Modeling, Materials, and Device Technology. Kluwer Academic Publishers, Boston/Dordrecht/London, 1998. 10, 11, 97, 98

[12] M.S. Keshner and R. Arya. Study of Potential Cost Reductions Resulting from Super-Large-Scale Manufacturing of PV Modules. Technical report, NREL, 2004. 10

[13] P. Campbell and M. A. Green. The Limiting Efficiency of Silicon Solar-Cells Under Concentrated Sunlight. IEEE Transactions on Electron Devices, 33(2): 234-239, 1986. 10, 98

[14] D. L. Staebler and C. R. Wronski. Reversible Conductivity Changes in Discharge-Produced Amorphous S. Applied Physics Letters, 31(4):292-294, 1977. 11, 98

[15] P. Lechner, W. Frammelsberger, W. Psyk, R. Geyer, H. Maurus, D. Lundszien, H. Wagner, and B. Eichhorn. Status of Performance of Thin Film Silicon Solar Cells and Modules. In 23rd European Photovoltaic Solar Energy Conference and Exhibition, pages 2023 - 2026, 2008. 11, 98

[16] E. Yablonovitch and G. D. Cody. Intensity Enhancement in Textured Optical Sheets for Solar-Cells. IEEE Transactions on Electron Devices, 29(2):300-305, 1982. $14,76,98$

[17] O. S. Heavens. Optical Properties of Thin Solid Films. Dover Publications, New York, 1983. 14, 55, 58

[18] M. D. Kelzenberg, S. W. Boettcher, J. A. Petykiewicz, D. B. Turner-Evans, M. C. Putnam, E. L. Warren, J. M. Spurgeon, R. M. Briggs, N. S. Lewis, and H. A. Atwater. Enhanced absorption and carrier collection in Si wire arrays for photovoltaic applications. Nature Materials, 9(3):239-244, 2010. 15, 145 
[19] M. Law, L. E. Greene, J. C. Johnson, R. Saykally, and P. D. Yang. Nanowire dye-sensitized solar cells. Nature Materials, 4(6):455-459, 2005. 15, 145

[20] J. Zhu, C.-M. Hsu, Z. Yu, S. Fan, and Y. Cui. Nanodome Solar Cells with Efficient Light Management and Self-Cleaning. Nano Letters, 10(6):1979-1984, 2010. $15,99,145$

[21] M. J. Naughton, K. Kempa, Z. F. Ren, Y. Gao, J. Rybczynski, N. Argenti, W. Gao, Y. Wang, Y. Peng, J. R. Naughton, G. McMahon, T. Paudel, Y. C. Lan, M. J. Burns, A. Shepard, M. Clary, C. Ballif, F. J. Haug, T. Soederstroem, O. Cubero, and C. Eminian. Efficient nanocoax-based solar cells. Physica Status Solidi-Rapid Research Letters, 4(7):181-183, JUL 2010. 15, 145, 160

[22] R. Biswas, J. Bhattacharya, B. Lewis, N. Chakravarty, and V. Dalal. Enhanced nanocrystalline silicon solar cell with a photonic crystal back-reflector. Solar Energy Materials and Solar Cells, 94(12):2337-2342, 2010. 15, 145

[23] S. B. Mallick, M. Agrawal, and P. Peumans. Optimal light trapping in ultra-thin photonic crystal crystalline silicon solar cells. Optics Express, 18(6):5691-5706, 2010. 15, 123, 145

[24] V. E. Ferry, M. A. Verschuuren, H. B. T. Li, E. Verhagen, R. J. Walters, R. E. I. Schropp, H. A. Atwater, and A. Polman. Light trapping in ultrathin plasmonic solar cells. Optics Express, 18(13):A237-A245, 2010. 15, 123, 145, 146, 160

[25] H. A. Atwater and A. Polman. Plasmonics for improved photovoltaic devices. Nature Materials, 9(3):205-213, 2010. 15, 99, 145

[26] O. Isabella, A. Campa, W. J. Heijna, M. Soppe, R. van Erven, R. H. Franken, H. Borg, and M. Zeman. Diffraction Gratings for Light Trapping in Thin-Film Silicon Solar Cells. In 23rd European Photovoltaic Solar Energy Conference and Exhibition, pages 2320 - 2324, 2008. 15, 99, 145

[27] C. Eisele, C. E. Nebel, and M. Stutzmann. Periodic light coupler gratings in 
188

amorphous thin film solar cells. Journal of Applied Physics, 89(12):7722-7726, 2001. $15,99,145,160$

[28] C. Haase and H. Stiebig. Thin-film silicon solar cells with efficient periodic light trapping texture. Applied Physics Letters, 91(6), 2007. 15, 99, 145, 160

[29] D. Shir, J. Yoon, D. Chanda, J.-H. Ryu, and J. A. Rogers. Performance of Ultrathin Silicon Solar Microcells with Nanostructures of Relief Formed by Soft Imprint Lithography for Broad Band Absorption Enhancement. Nano Letters, 10(8):3041-3046, 2010. 15, 145

[30] Z. Yu, A. Raman, and S. Fan. Fundamental limit of nanophotonic light trapping in solar cells. Proceedings of the National Academy of Sciences of the United States of America, 107(41):17491-17496, 2010. 15

[31] Z. Yu, A. Raman, and S. Fan. Fundamental limit of light trapping in grating structures. Optics Express, 18(19):A366-A380, 2010. 15

[32] P. N. Saeta, V. E. Ferry, D. Pacifici, J. N. Munday, and H. A. Atwater. How much can guided modes enhance absorption in thin solar cells? Optics Express, 17(23):20975-20990, 2009. 15, 23, 26, 27, 28, 99

[33] G. Mie. Beiträge zur optik trüber medien, speziell kolloidaler metallösungen. Annalen Der Physik, 25(3):377-445, 1908. 16

[34] R. H. Ritchie. Plasma losses by fast electrons in thin films. Physical Review, 106, 1957. 16

[35] A. Otto. Exictation of Nonradiative Surface Plasma Waves in Silver by Method of Frustrated Total Reflection. Zeitschrift fur Physik, 216(4):398-\&, 1968. 16, 53

[36] E. Kretschmann and H. Raether. Radiative decay of non-radiative surface plasmons excited by light. Zeitschrift fur Naturforschung, 23A, 1968. 16 
[37] D. L. Jeanmaire and R. P. Van Duyne. Surface raman spectroelectrochemistry: Part i. heterocyclic, aromatic, and aliphatic amines adsorbed on the anodized silver electrode. Journal of Electroanalytical Chemistry, 84:1-20, 1977. 16

[38] K. A. Willets and R. P. Van Duyne. Localized surface plasmon resonance spectroscopy and sensing. Annual Review of Physical Chemistry, 58:267-297, 2007. 16

[39] A. Polman. Applied Physics Plasmonics Applied. Science, 322(5903):868-869, 2008. 16

[40] J. Mertz. Radiative absorption, fluorescence, and scattering of a classical dipole near a lossless interface: a unified description. Journal of the Optical Society of America B-Optical Physics, 17(11):1906-1913, 2000. 16, 78

[41] J. B. Pendry. Negative refraction makes a perfect lens. Physical Review Letters, 85(18):3966-3969, 2000. 16

[42] R. J. Walters, R. V. A. van Loon, I. Brunets, J. Schmitz, and A. Polman. A silicon-based electrical source of surface plasmon polaritons. Nature Materials, $9(1): 21-25,2010.16$

[43] R. F. Oulton, V. J. Sorger, T. Zentgraf, R.-M. Ma, C. Gladden, L. Dai, G. Bartal, and X. Zhang. Plasmon lasers at deep subwavelength scale. Nature, 461 (7264):629-632, 2009. 16

[44] M. T. Hill, Y.-S. Oei, B. Smalbrugge, Y. Zhu, T. De Vries, P. J. Van Veldhoven, F. W. M. Van Otten, T. J. Eijkemans, J. P. Turkiewicz, H. De Waardt, E. J. Geluk, S.-H. Kwon, Y.-H. Lee, R. Notzel, and M. K. Smit. Lasing in metallicCoated nanocavities. Nature Photonics, 1(10):589-594, 2007. 16

[45] S. Lal, S. E. Clare, and N. J. Halas. Nanoshell-Enabled Photothermal Cancer Therapy: Impending Clinical Impact. Accounts of Chemical Research, 41(12, Sp. Iss. SI):1842-1851, 2008. 16 
[46] S. Pillai, K. R. Catchpole, T. Trupke, and M. A. Green. Surface plasmon enhanced silicon solar cells. Journal of Applied Physics, 101(9), 2007. 17, 18, $22,72,81,99,100$

[47] D. Derkacs, S. H. Lim, P. Matheu, W. Mar, and E. T. Yu. Improved performance of amorphous silicon solar cells via scattering from surface plasmon polaritons in nearby metallic nanoparticles. Applied Physics Letters, 89(9), 2006. 17, 72, 99,100

[48] K. Nakayama, K. Tanabe, and H. A. Atwater. Plasmonic nanoparticle enhanced light absorption in GaAs solar cells. Applied Physics Letters, 93(12), 2008. 17, $18,22,72,99,100$

[49] R. B. Konda, R. Mundle, H. Mustafa, O. Bamiduro, A. K. Pradhana, U. N. Roy, Y. Cui, and A. Burger. Surface plasmon excitation via Au nanoparticles in n-CdSe/p-Si heterojunction diodes. Applied Physics Letters, 91(19), 2007. 17

[50] I. M. Pryce, D. D. Koleske, A. J. Fischer, and H. A. Atwater. Plasmonic nanoparticle enhanced photocurrent in $\mathrm{GaN} / \mathrm{InGaN} / \mathrm{GaN}$ quantum well solar cells. Applied Physics Letters, 96(15), 2010. 17, 18, 99, 100

[51] D. Derkacs, W. V. Chen, P. M. Matheu, S. H. Lim, P. K. L. Yu, and E. T. Yu. Nanoparticle-induced light scattering for improved performance of quantumwell solar cells. Applied Physics Letters, 93(9), 2008. 17, 18

[52] A. J. Morfa, K. L. Rowlen, T. H. Reilly, III, M. J. Romero, and J. van de Lagemaat. Plasmon-enhanced solar energy conversion in organic bulk heterojunction photovoltaics. Applied Physics Letters, 92(1), 2008. 17

[53] M. Westphalen, U. Kreibig, J. Rostalski, H. Luth, and D. Meissner. Metal cluster enhanced organic solar cells. Solar Energy Materials and Solar Cells, 61 (1):97-105, 2000. 17 
[54] C. Wen, K. Ishikawa, M. Kishima, and K. Yamada. Effects of silver particles on the photovoltaic properties of dye-sensitized $\mathrm{TiO} 2$ thin films. Solar Energy Materials and Solar Cells, 61(4):339-351, 2000. 17

[55] P. Spinelli, M. Hebbink, R. de Waele, L. Black, F. Lenzmann, and A. Polman. Optical impedance matching using coupled plasmonic nanoparticle arrays. Nano Letters, 11(4):1760-1765, 2011. 17

[56] J. N. Munday and H. A. Atwater. Large integrated absorption enhancement in plasmonic solar cells by combining metallic gratings and antireflection coatings. Nano Letters, 2011. doi: 10.1021/nl101875t. 17

[57] H. R. Stuart and D. G. Hall. Island size effects in nanoparticle-enhanced photodetectors. Applied Physics Letters, 73(26):3815-3817, 1998. 18, 23, 71, 99, 100

[58] H. R. Stuart and D. G. Hall. Enhanced dipole-dipole interaction between elementary radiators near a surface. Physical Review Letters, 80(25):5663-5666, 1998. 18

[59] H. R. Stuart and D. G. Hall. Absorption enhancement in silicon-on-insulator waveguides using metal island films. Applied Physics Letters, 69(16):2327-2329, 1996. 18

[60] P. Matheu, S. H. Lim, D. Derkacs, C. McPheeters, and E. T. Yu. Metal and dielectric nanoparticle scattering for improved optical absorption in photovoltaic devices. Applied Physics Letters, 93(11), 2008. 18, 99, 100

[61] D. M. Schaadt, B. Feng, and E. T. Yu. Enhanced semiconductor optical absorption via surface plasmon excitation in metal nanoparticles. Applied Physics Letters, 86(6), 2005. 18

[62] B. P. Rand, P. Peumans, and S. R. Forrest. Long-range absorption enhancement in organic tandem thin-film solar cells containing silver nanoclusters. Journal of Applied Physics, 96(12):7519-7526, 2004. 18, 22 
[63] M. Kirkengen, J. Bergli, and Y. M. Galperin. Direct generation of charge carriers in c-Si solar cells due to embedded nanoparticles. Journal of Applied Physics, 102(9), 2007. 18

[64] C. Hagglund, M. Zach, and B. Kasemo. Enhanced charge carrier generation in dye sensitized solar cells by nanoparticle plasmons. Applied Physics Letters, 92 (1), 2008. 18

[65] S.-S. Kim, S.-I. Na, J. Jo, D.-Y. Kim, and Y.-C. Nah. Plasmon enhanced performance of organic solar cells using electrodeposited Ag nanoparticles. Applied Physics Letters, 93(7), 2008. 18

[66] H. Masuda and M. Satoh. Fabrication of gold nanodot array using anodic porous alumina as an evaporation mask. Japanese Journal of Applied Physics Part 2-Letters, 35(1B):L126-L129, 1996. 18

[67] V. E. Ferry, L. A. Sweatlock, D. Pacifici, and H. A. Atwater. Plasmonic Nanostructure Design for Efficient Light Coupling into Solar Cells. Nano Letters, 8 (12):4391-4397, 2008. 23, 72, 99

[68] R. A. Pala, J. White, E. Barnard, J. Liu, and M. L. Brongersma. Design of Plasmonic Thin-Film Solar Cells with Broadband Absorption Enhancements. Advanced Materials, 21(34):3504+, 2009. 23

[69] K. R. Catchpole and A. Polman. Design principles for particle plasmon enhanced solar cells. Applied Physics Letters, 93(19), 2008. 24, 26, 99, 106

[70] K. Tanabe, K. Nakayama, and H. A. Atwater. Plasmon-enhanced absorption and photocurrent in ultrathin gaas solar cells with metallic nanostructures. In PVSC: 2008 33rd IEEE Photovoltaic Specialists Conference, VOLS 1-4, IEEE Photovoltaic Specialists Conference, pages 491-494, 2008. 33rd IEEE Photovoltaic Specialists Conference, San Diego, CA, MAY 11-16, 2008. 24

[71] C.F. Bohren and D. R. Huffman. Absorption and Scattering of Light by Small Particles. John Wiley \& Sons, New York, 1983. 25, 57, 72, 91 
193

[72] A. Taflove and S. C. Hagness. Computational Electrodynamics: The FiniteDifference Time-Domain Method. Artech House, Norwood, Massachusetts, 3rd edition, 2005. 35, 123

[73] S. G. Johnson, M. L. Povinelli, P. Bienstman, M. Skorobogatiy, M. Soljacic, M. Ibanescu, E. Lidorikis, and J. D. Joannopoulos. Coupling, scattering, and perturbation theory: Semi-analytical analyses of photonic-crystal waveguides. In M Marciniak, editor, ICTON 2003: 5TH International Conference on Transparent Optical Networks, Vol 1, Proceedings, pages 103-109, 2003. 36

[74] A. Yariv. Coupled-mode theory for guided-wave optics. IEEE Journal of Quantum Electronics, QE 9(9):919-933, 1973. 36

[75] A.-L. Baudrion, F. de Leon-Perez, O. Mahboub, A. Hohenau, H. Ditlbacher, F. J. Garcia-Vidal, J. Dintinger, T. W. Ebbesen, L. Martin-Moreno, and J. R. Krenn. Coupling efficiency of light to surface plasmon polariton for single subwavelength holes in a gold film. Optics Express, 16(5):3420-3429, 2008. 36, 53

[76] H. W. Kihm, K. G. Lee, D. S. Kim, J. H. Kang, and Q-Han Park. Control of surface plasmon generation efficiency by slit-width tuning. Applied Physics Letters, 92(5), 2008. 36, 53

[77] G. Leveque, O. J. F. Martin, and J. Weiner. Transient behavior of surface plasmon polaritons scattered at a subwavelength groove. Physical Review B, 76 (15), 2007. 36,53

[78] A. D. Rakic, A. B. Djurisic, J. M. Elazar, and M. L. Majewski. Optical properties of metallic films for vertical-cavity optoelectronic devices. Applied Optics, $37(22): 5271-5283,1998.37,124$

[79] E. Palik and G. Ghosh. Handbook of Optical Constants of Solids, Volume 3. Academic Press, New York, 1997. 37, 124 
[80] M. Born and E. Wolf. Principles of Optics. Cambridge University Press, Cambridge, 1999. 49, 75, 85

[81] E. Kretschmann. Determination of Optical Constants of Metals by Excitation of Surface Plasmons. Zeitschrift fur Physik, 241(4):313-\&, 1971. 53

[82] R. H. Ritchie, E. T. Arakawa, J. J. Cowan, and R. N. Hamm. Surface-Plasmon Resonance Effect in Grating Diffraction. Physical Review Letters, 21(22):1530\&, 1968. 53

[83] S. H. Chang, S. K. Gray, and G. C. Schatz. Surface plasmon generation and light transmission by isolated nanoholes and arrays of nanoholes in thin metal films. Optics Express, 13(8):3150-3165, 2005. 53

[84] D. Pacifici, H. J. Lezec, H. A. Atwater, and J. Weiner. Quantitative determination of optical transmission through subwavelength slit arrays in Ag films: Role of surface wave interference and local coupling between adjacent slits. Physical Review B, 77(11), 2008. 53

[85] D. Pacifici, H. J. Lezec, L. A. Sweatlock, R. J. Walters, and H. A. Atwater. Universal optical transmission features in periodic and quasiperiodic hole arrays. Optics Express, 16(12):9222-9238, 2008. 53

[86] T. H. Isaac, J. Gomez Rivas, J. R. Sambles, W. L. Barnes, and E. Hendry. Surface plasmon mediated transmission of subwavelength slits at $\mathrm{THz}$ frequencies. Physical Review B, 77(11), 2008. 53

[87] J. R. Suckling, A. P. Hibbins, M. J. Lockyear, T. W. Preist, J. R. Sambles, and C. R. Lawrence. Finite conductance governs the resonance transmission of thin metal slits at microwave frequencies. Physical Review Letters, 92(14), 2004. 53

[88] D. Pacifici, H. J. Lezec, and H. A. Atwater. All-optical modulation by plasmonic excitation of CdSe quantum dots. Nature Photonics, 1(7):402-406, 2007. 53 
[89] J. S. Q. Liu and M. L. Brongersma. Omnidirectional light emission via surface plasmon polaritons. Applied Physics Letters, 90(9), 2007. 53

[90] J. A. Dionne, E. Verhagen, A. Polman, and H. A. Atwater. Are negative index materials achievable with surface plasmon waveguides? A case study of three plasmonic geometries. Optics Express, 16(23):19001-19017, 2008. 54

[91] Y. Kurokawa and H. T. Miyazaki. Metal-insulator-metal plasmon nanocavities: Analysis of optical properties. Physical Review B, 75(3), 2007. 57

[92] A. D. Yaghjian. Internal energy, Q-energy, Poynting's theorem, and the stress dyadic in dispersive material. IEEE Transactions on Antennas and Propagation, 55:1495-1505, 2007. 63

[93] W. Shockley and H. J. Queisser. Detailed Balance Limit of Efficiency of P-N Junction Solar Cells. Journal of Applied Physics, 32(3):510-\&, 1961. 71

[94] C. H. Henry. Limiting Efficiencies of Ideal Single and Multiple Energy-Gap Terrestrial Solar-Cells. Journal of Applied Physics, 51(8):4494-4500, 1980. 71

[95] K. Schick, E. Daub, S. Finkbeiner, and P. Wurfel. Verification of a generalized planck law for luminescence radiation from silicon solar-cells. Applied Physics A-Materials Science \& Processing, 54(2):109-114, 1992. 71

[96] P. Campbell and M. A. Green. Light Trapping Properties of Pyramidally Textured Surfaces. Journal of Applied Physics, 62(1):243-249, 1987. 71

[97] S. S. Hegedus and X. M. Deng. Analysis of optical enhancement in a-Si n-i-p solar cells using a detachable back reflector. In Conference Record of the Twenty Fifth IEEE Photovoltaic Specialists Conference - 1996, pages 1061-1064, 1996. 71

[98] H. R. Stuart and D. G. Hall. Thermodynamic limit to light trapping in thin planar structures. Journal of the Optical Society of America A-Optics Image Science and Vision, 14(11):3001-3008, 1997. 71, 87 
[99] B. J. Soller and D. G. Hall. Energy transfer at optical frequencies to siliconbased waveguiding structures. Journal of the Optical Society of America AOptics Image Science and Vision, 18(10):2577-2584, OCT 2001. ISSN 07403232. 71

[100] K. R. Catchpole and S. Pillai. Absorption enhancement due to scattering by dipoles into silicon waveguides. Journal of Applied Physics, 100(4), 2006. 72, $78,92,123$

[101] F. J. Beck, A. Polman, and K. R. Catchpole. Tunable light trapping for solar cells using localized surface plasmons. Journal of Applied Physics, 105(11), 2009. $72,99,106$

[102] T. Tiedje, E. Yablonovitch, G. D. Cody, and B. G. Brooks. Limiting Efficiency of Silicon Solar-Cells. IEEE Transactions on Electron Devices, 31(5):711-716, 1984. 76

[103] P. Sheng. Optical-Absorption of Thin-Film on a Lambertian Reflector Substrate. IEEE Transactions on Electron Devices, 31(5):634-636, 1984. 76

[104] J. E. Sipe. The Dipole Antenna Problem in Surface Physics - a New Approach. Surface Science, 105(2-3):489-504, 1981. 78

[105] G. W. Ford and W. H. Weber. Electromagnetic-Interactions of Molecules with Metal-Surfaces. Physics Reports-Review Section of Physics Letters, 113(4):195287, 1984. 78

[106] H. Benisty, R. Stanley, and M. Mayer. Method of source terms for dipole emission modification in modes of arbitrary planar structures. Journal of the Optical Society of America A-Optics Image Science and Vision, 15(5):11921201, 1998. 78

[107] W. L. Barnes. Fluorescence near interfaces: the role of photonic mode density. Journal of Modern Optics, 45(4):661-699, 1998. 78 
[108] A. C. Hryciw, Y. C. Jun, and M. L. Brongersma. Plasmon-enhanced emission from optically-doped MOS light sources. Optics Express, 17(1):185-192, 2009. 78

[109] F. Leblanc, J. Perrin, and J. Schmitt. Numerical Modeling of the OpticalProperties of Hydrogenated Amorphous-Silicon-Based P-I-N Solar-Cells Deposited on Rough Transparent Conducting Oxide Substrates. Journal of Applied Physics, 75(2):1074-1087, 1994. 81, 83

[110] D. Poitras and J. A. Dobrowolski. Toward perfect antireflection coatings. 2. Theory. Applied Optics, 43(6):1286-1295, 2004. 85

[111] M.-L. Kuo, D. J. Poxson, Y. S. Kim, F. W. Mont, L. K. Kim, E. F. Schuhert, and S.-Y. Lin. Realization of a near-perfect antireflection coating for silicon solar energy utilization. Optics Letters, 33(21):2527-2529, 2008. 85

[112] A. V. Shah, H. Schade, M. Vanecek, J. Meier, E. Vallat-Sauvain, N. Wyrsch, U. Kroll, C. Droz, and J. Bailat. Thin-film silicon solar cell technology. Progress in Photovoltaics, 12(2-3):113-142, 2004. 98

[113] U. Dagkaldiran, A. Gordijn, F. Finger, H. M. Yates, P. Evans, D. W. Sheel, Z. Remes, and M. Vanecek. Amorphous silicon solar cells made with SnO2:F TCO films deposited by atmospheric pressure CVD. Materials Science and Engineering B-Advanced Functional Solid-State Materials, 159-60(Sp. Iss. SI): 6-9, 2009. 99

[114] J. Krc, F. Smole, and M. Topic. Potential of light trapping in microcrystalline silicon solar cells with textured substrates. Progress in Photovoltaics, 11(7): 429-436, 2003. 99

[115] S. Fahr, C. Rockstuhl, and F. Lederer. Engineering the randomness for enhanced absorption in solar cells. Applied Physics Letters, 92(17), 2008. 99

[116] R. H. Franken, R. L. Stolk, H. Li, C. H. M. van der Werf, J. K. Rath, and R. E. I. Schropp. Understanding light trapping by light scattering textured 
back electrodes in thin film n-i-p-type silicon solar cells. Journal of Applied Physics, 102(1), 2007. 99, 145

[117] J. Muller, B. Rech, J. Springer, and M. Vanecek. TCO and light trapping in silicon thin film solar cells. Solar Energy, 77(6):917-930, 2004. 99, 145

[118] F-J. Haug, T. Soederstroem, O. Cubero, V. Terrazzoni-Daudrix, and C. Ballif. Plasmonic absorption in textured silver back reflectors of thin film solar cells. Journal of Applied Physics, 104(6), 2008. 99

[119] K. Niira, H. Senta, H. Hakuma, M. Komoda, H. Okui, K. Fukui, H. Arimune, and K. Shirasawa. Thin film poly-Si solar cells using PECVD and Cat-CVD with light confinement structure by RIE. Solar Energy Materials and Solar Cells, 74(1-4):247-253, 2002. 99

[120] R. E. I. Schropp, J. K. Rath, and H. Li. Growth mechanism of nanocrystalline silicon at the phase transition and its application in thin film solar cells. Journal of Crystal Growth, 311(3):760-764, 2009. 99

[121] V. E. Ferry, J. N. Munday, and H. A. Atwater. Design Considerations for Plasmonic Photovoltaics. Advanced Materials, 22(43):4794-4808, 2010. 99

[122] A. Lin and J. Phillips. Optimization of random diffraction gratings in thin-film solar cells using genetic algorithms. Solar Energy Materials and Solar Cells, 92 (12):1689-1696, 2008. 99

[123] V. E. Ferry, M. A. Verschuuren, H. B. T. Li, R. E. I. Schropp, H. A. Atwater, and A. Polman. Improved red-response in thin film a-Si:H solar cells with softimprinted plasmonic back reflectors. Applied Physics Letters, 95(18), 2009. 100

[124] C. Stuart and Y. Chen. Roll in and Roll out: A Path to High-Throughput Nanoimprint Lithography. ACS Nano, 3(8):2062-2064, 2009. 100, 146

[125] M. Beck, M. Graczyk, I. Maximov, E. L. Sarwe, T. G. I. Ling, M. Keil, and L. Montelius. Improving stamps for $10 \mathrm{~nm}$ level wafer scale nanoimprint lithography. Microelectronic Engineering, 61-2:441-448, 2002. 100 
[126] G. Y. Jung, Z. Y. Li, W. Wu, Y. Chen, D. L. Olynick, S. Y. Wang, W. M. Tong, and R. S. Williams. Vapor-phase self-assembled monolayer for improved mold release in nanoimprint lithography. Langmuir, 21(4):1158-1161, 2005. 100

[127] M. A. Verschuuren. Substrate Conformal Imprint Lithography for Nanophotonics. PhD thesis, Utrecht University, 2010. 100

[128] T. W. Odom, J. C. Love, D. B. Wolfe, K. E. Paul, and G. M. Whitesides. Improved pattern transfer in soft lithography using composite stamps. Langmuir, 18(13):5314-5320, 2002. 101

[129] M. Verschuuren and H. van Sprang. 3D Photonic Structures by Sol-Gel Imprint Lithography. In 2007 Material Research Society Symposium Proceedings Vol. 1002, Materials Research Society, pages N03-05. MRS, 2007. 102, 146

[130] H. B. T. Li, C. H. M. van der Werf, J. K. Rath, and R. E. I. Schropp. Hot wire CVD deposition of nanocrystalline silicon solar cells on rough substrates. Thin Solid Films, 517(12):3476-3480, 2009. 110

[131] C. Rockstuhl, S. Fahr, K. Bittkau, T. Beckers, R. Carius, F. J. Haug, T. Soederstroem, C. Ballif, and F. Lederer. Comparison and optimization of randomly textured surfaces in thin-film solar cells. Optics Express, 18(19):A335-A342, 2010. 123,145

[132] A. Naqavi, K. Soederstroem, F.-J. Haug, V. Paeder, T. Scharf, H. P. Herzig, and C. Ballif. Understanding of photocurrent enhancement in real thin film solar cells: towards optimal one-dimensional gratings. Optics Express, 19(1): 128-140, 2011. 123

[133] M. Zeman, R. A. C. M. M. van Swaaij, J. W. Metselaar, and R. E. I. Schropp. Optical modeling of a-Si : $\mathrm{H}$ solar cells with rough interfaces: Effect of back contact and interface roughness. Journal of Applied Physics, 88(11):6436-6443, 2000. 123 
[134] J. Kim, A. Abou-Kandil, K. Fogel, H. Hovel, and D. K. Sadana. The Role of High Work-Function Metallic Nanodots on the Performance of a-Si:H Solar Cells: Offering Ohmic Contact to Light Trapping. ACS Nano, 4(12):7331-7336, 2010. 131

[135] G. Yue, L. Sivec, J. M. Owens, B. Yan, J. Yang, and S. Guha. Optimization of back reflector for high efficiency hydrogenated nanocrystalline silicon solar cells. Applied Physics Letters, 95(26), 2009. 145

[136] H. Sai and M. Kondo. Effect of self-orderly textured back reflectors on light trapping in thin-film microcrystalline silicon solar cells. Journal of Applied Physics, 10(9), 2009. 145

[137] K. Soederstroem, J. Escarre, O. Cubero, F-J. Haug, S. Perregaux, and C. Ballif. UV-nano-imprint lithography technique for the replication of back reflectors for n-i-p thin film silicon solar cells. Progress in Photovoltaics, 19(2):202-210, 2011. 146

[138] C. Battaglia, J. Escarre, K. Soederstroem, L. Erni, L. Ding, G. Bugnon, A. Billet, M. Boccard, L. Barraud, S. De Wolf, F.-J. Haug, M. Despeisse, and C. Ballif. Nanoimprint Lithography for High-Efficiency Thin-Film Silicon Solar Cells. Nano Letters, 11(2):661-665, 2011. 146, 152

[139] F. Biscarini, P. Samori, O. Greco, and R. Zamboni. Scaling behavior of anisotropic organic thin films grown in high vacuum. Physical Review Letters, 78(12):2389-2392, 1997. 152

[140] Y.-F. Huang, S. Chattopadhyay, Y.-J. Jen, C.-Y. Peng, T.-A. Liu, Y.-K. Hsu, C.-L. Pan, H.-C. Lo, C.-H. Hsu, Y.-H. Chang, C.-S. Lee, K.-H. Chen, and L.-C. Chen. Improved broadband and quasi-omnidirectional anti-reflection properties with biomimetic silicon nanostructures. Nature Nanotechnology, 2(12):770-774, 2007. 160 